\title{
Physics Basis for Optical Performance of the NIF Amplifiers
}

January 11, 1999 


\section{DISCLAIMER}

This document was prepared as an account of work sponsored by an agency of the United States Government. Neither the United States Government nor the University of Califormia nor any of their employees, makes any warranty, express or implied, or assumes any legal liability or responsibility for the accuracy, completeness, or usefulness of any information, apparatus, product, or process disclosed, or represents that its use would not infringe privately owned rights. Reference herein to any specific commercial products, process, or service by trade name, trademark, manufacturer, or otherwise, does not necessarily constitute or imply its endorsement, recommendation, or favoring by the United States Government or the University of California. The views and opinions of authors expressed herein do not necessarily state or reflect those of the United States Government or the University of California, and shall not be used for advertising or product endorsement purposes.

This report has been reproduced directly from the best available copy.

Available to DOE and DOE contractors from the

Office of Scientific and Technical Information

P.O. Box 62, Oak Ridge, TN 37831

Prices available from (615) 576-8401, FTS 626-8401

Available to the public from the

National Technical Information Service

U.S. Department of Commerce

5285 Port Royal Rd.,

Springfield, VA 22161

Work performed under the auspices of the U.S. Department of Energy by Lawrence Livermore National Laboratory under Contract W-7405-Eng-48. 


\section{Physics Basis for Optical Performance of the NIF Amplifiers}

\section{Contributors and Authors}

Core Development

Terry Alger

Al Eriandson * ${ }^{\dagger}$

Steve Fulkerson*

John Horvath

Ken Jancaitis *

Chris Marshall*

Mark Rotter*

Steve Sutton*

Luis Zapata*

Additional analysis

Peter Amendt

Janice Lawson*

Richard London

David Milam

Mark Newton

Ray Pierce

Rick Sacks

Wade Williams*

Yury Zakharenkov

Advisors

John Hunt

Ken Manes

John Murray

John Trenholme

Mangoement Participants

Richard Hackel

Douglas Larson

Jeffrey Paisner

Stephen Payne*

Lawrence Pedrotti

Howard Powell

Coordinators

Larry Morris

Greg Rogowski

Laser slabs

Jack Campbell

William Steele

Dan Walmer

*Authors

† Scientific Editor
French Collaborators

Thierry Adjadj

Jacques Beullier

Thierry Berthier

Beatrice Bicrel

Olivier Carbourdin

Eric Grebot

Jean-Luc Guenet

Mireille Guenet

Fabrice Laniesse

Geoffroy LeTouzé

Jean Lobit

Xavier Maille

Patrick Manac'h

Jean-Francois Mengué

Jean Marie Morchain

Stéphane Seznec

Mechanical Design

Ron Bettencourt

Neil Frank

Ed Hajdena

John Horvath

Loretta Kleinsasser

Tony Lee

Ernie Moor

Dzung Nguyen

Sam Rodriguez

Doug Swort

Mike Weddle

Coatings

Dave Ahre

Jerry Britten

Joe Farmer

Dave Fix

Hugh Gregg

Curly Hoaglan

Ton Parham

Wigbert Siekhaus

Norman Thomas
Technical

Contributors

Randy Aceves

Michael Assen

Robert Akusis

Cindy Alviso

Ron Cabeceiras

Wilbert Davis

Alex Drobshoff

Jerome Hall

Drew Hargiss

Steve Letts

Richard McCracken

Rene Neurath

Charles Petty

Gary Ross

Dennis Silva

Joseph Smith

Larry Smith

John Toeppen

John Trent

Steve Weller

Adminstration

Lori Dempsey

Annette Springer

Cidelia Sanchez

Power Conditioning

Andrew Hinz

Scott Hulsey

Douglas Larson

Phil Test

Technical Information

Department Editor

Cindy Cassady

January 11, 1999

LAWRENCE LIVERMORE NATIONAL LABORATORY University of California • Livermore, California • 94550 


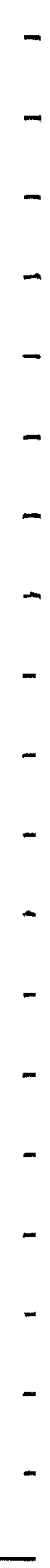




\section{Table of Contents}

EXECUTIVE SUMMARY 1

\subsection{OPTICAL PERFORMANCE REQUIREMENTS FOR THE NIF AMPLIFIERS} 7

1.1 Formal requirements

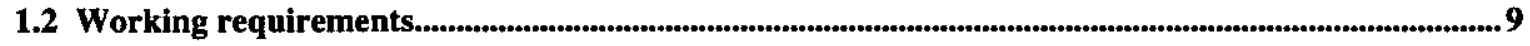

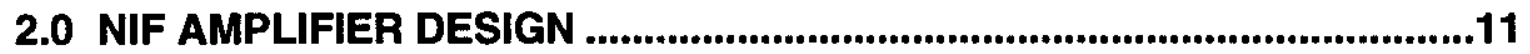

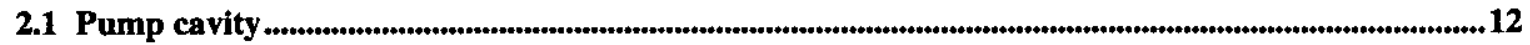

2.2 Thermal management

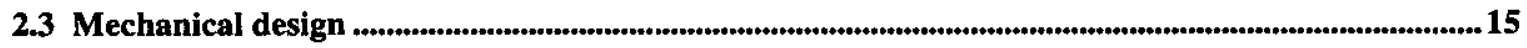

3.0 DESCRIPTION OF NIF PROTOTYPE AMPLIFIER AND EXPERIMENTS .17

3.1 Description of prototype amplifier and comparison with the NIF Title II baseline

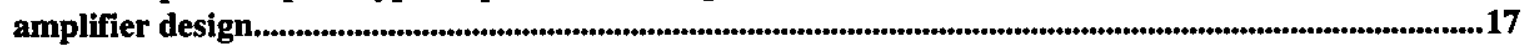

3.2 Large-aperture diagnostic system (LADS) ……...................................................................................22

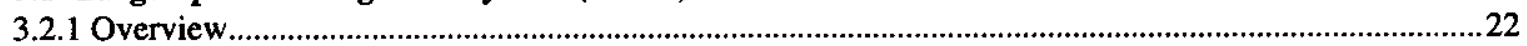

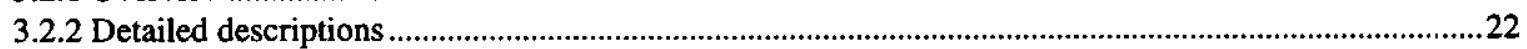

3.3 Test configurations

3.4 Power conditioning system

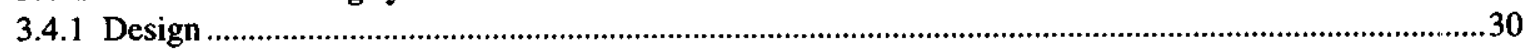

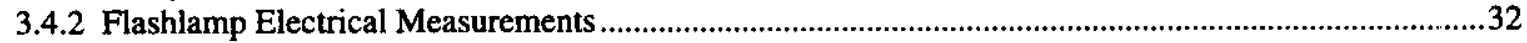

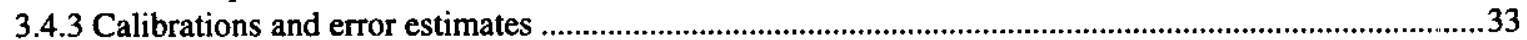

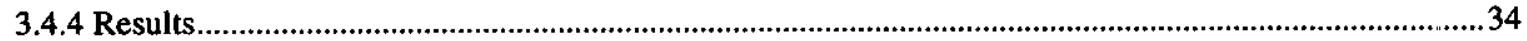

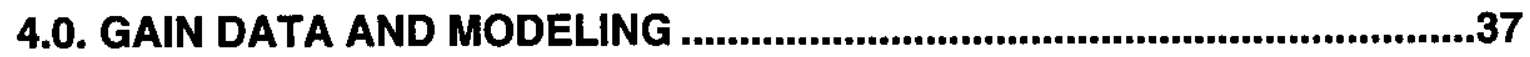

4.1 Gain data reduction

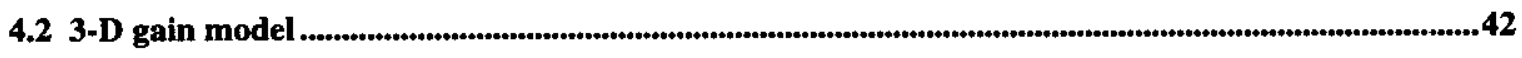

4.3 Comparison of gain measurements to the 3D model predictions..............................................................43

4.3.1 Validation of the assumptions made in building the amplifier model .....................................................44

4.3.2 Comparison of the measurements and model predictions for both end and interior slabs.....................45

4.3.3 Comparison of the measurements and model predictions for the $\mathrm{D}$ (bottom) aperture...........................56

4.3.4 Comparison of the measurements and model predictions for LHG-8 glass in the

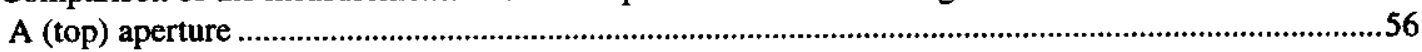

4.3.5 Comparison of the measurements and model predictions for different explosion fractions ...................61

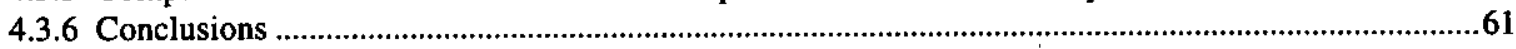

4.4 Gain predictions....................................................................................................................................66 


\subsection{PROMPT PUMP-INDUCED WAVEFRONT MEASUREMENTS}

AND MODELING ............................................................................................

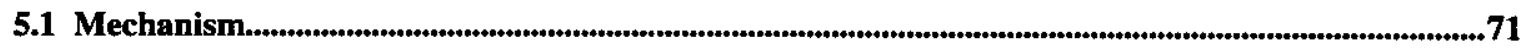

5.2 Data analysis method

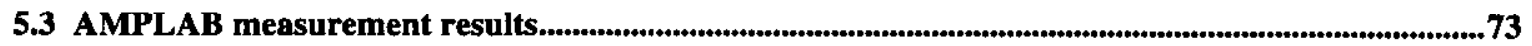

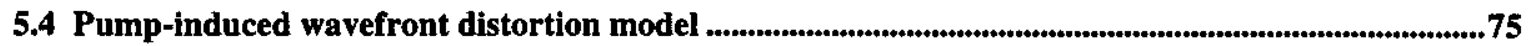

5.5 Comparison of model predictions with AMPLAB measurements ...........................................................81

5.6 Comparison of model predictions with Beamlet measurements ...................................................................88

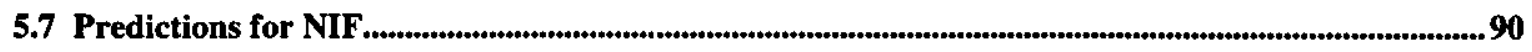

5.8 Summary and conclusions for prompt pump-induced wavefront distortion .........................................97

\subsection{THERMAL RECOVERY MEASUREMENTS AND MODELING ...................99}

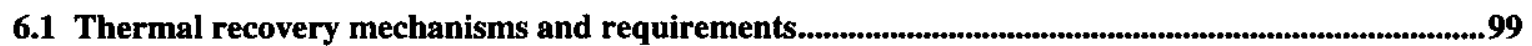

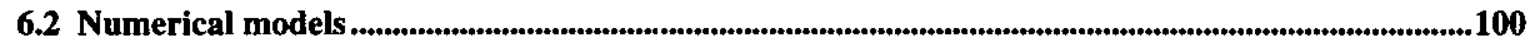

6.3 AMPLAB temperature measurements.....................................................................................................103

6.4 Comparison of AMPLAB temperature measurements with model predictions.....................................107

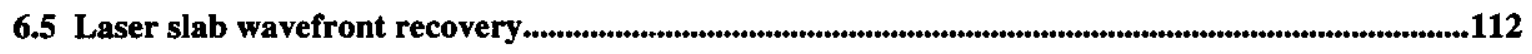

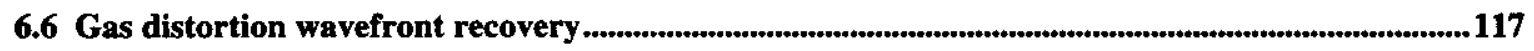

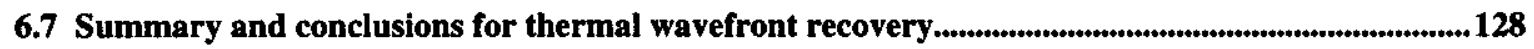

7.0 IMPLICATIONS FOR NIF PERFORMANCE .......................................131

8.0 DISCUSSION AND RECOMMENDATIONS.............................................137

8.1 Gain and gain uniformity

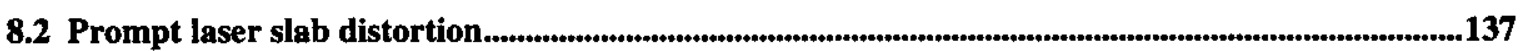

8.3 Long-term thermal distortion ................................................................................................................138

REFERENCES....................................................................................................139

APPENDIX A- CALCULATION OF SLAB PROMPT

TEMPERATURE RISE ........................................................................................ 


\section{Executive Summary}

This document describes extensive experiments and modeling performed during the late 1990s at Lawrence Livermore National Laboratory (LLNL) to characterize the optical performance of flashlamp-pumped, Nd:glass amplifiers for the National Ignition Facility (NIF). These analyses were undertaken to address three major aspects of amplifier performance: gain and gain uniformity, prompt pump-induced wavefront distortion, and long-term thermal wavefront recovery. The experiments consisted of performance measurements made on the NIF prototype amplifier, a four-aperture-high $x$ two-aperture-wide multisegment amplifier with $40-\mathrm{cm}$-square apertures. This prototype amplifier was nearly identical to the NIF amplifier design, with the same size flashlamps and laser slabs, nearly the same reflector shapes, similar antireflective (AR) coatings on the blastshields to improve pumping efficiency, and flowing gas to cool the flashlamps and to accelerate thermal wavefront recovery after shots. The prototype amplifier was tested as a one-, two-, and three-slab-long amplifier, so that the results could be extrapolated to accurately predict the performance of the five- and eleven-slab-long NIF amplifiers. Although the dimensions, internal positions, and shapes of the components in the NIF production amplifiers will be slightly different from the prototype, these differences were small, and our analyses suggest that only slight differences in performance may be expected.

Table E. 1 summarizes the NIF amplifier performance requirements and our performance predictions. It also describes the basis for each prediction as well as implications, caveats, and recommendations.

Our experiments and modeling predict that the NIF amplifiers will meet seven of their twelve specific optical performance requirements. These seven requirements are average gain coefficient, prompt pump-induced steering of the beam centroid, shot-toshot variations in pump-induced wavefront distortion, slab thermal distortion, slab thermal-distortion drift one hour prior to shot, gas thermal distortions, and pump-induced depolarization. The gain predictions presented in this report are based on 3-D ray-trace modeling of the NIF prototype amplifier. Modeling of the NIF Title-II baseline design, which is now underway, will be described in an addendum as soon as results become available.

To date our analysis has not verified three of the twelve requirements. These three requirements are drift of the beam centroid due to varying temperature gradients in the laser slabs, mounting-stress-induced wavefront distortion, and mounting-induced depolarization. The first of these three requirements appears to present little performance risk to the laser system and will be validated on the NIF first bundle. Mounting-stressinduced wavefront distortion and depolarization pose significant potential risk to the laser system, however. Measurements of mounting-stress-induced wavefront distortion and depolarization using an engineering prototype slab holder were underway as this report was being written. The results of these important tests were not available in time for inclusion in this report, however. 
Our analyses show that two of the twelve optical performance requirements, as currently written, will not be met. These two requirements are gain uniformity and prompt pump-induced wavefront distortion. The measure of gain uniformity used in the NIF requirements is the peak-to-average ratio, $\alpha_{\text {peak }} /\langle\alpha\rangle$. Our predicted value of $1.08 \pm$ 0.02 exceeds the NIF maximum allowed value of 1.05 , when the flashlamps are fired at $20 \%$ of their single-shot explosion energy. However, the predicted average gain coefficient was $5.23 \%$, exceeding the minimum $5 \% / \mathrm{cm}$ requirement by nearly $5 \%$. Since gain uniformity is strongly affected by amplified spontaneous emission, the predicted peak-to-average ratio will be closer to meeting the requirement when flashlamp energy is reduced to just meet the $5.0 \% / \mathrm{cm}$ requirement. We recommend that amplifier and propagation modeling be undertaken to evaluate this case, as well as to study tradeoffs between gain, gain uniformity, and input-fluence shapes, and that gain and gainuniformity requirements be revised for optimized system performance.

The predicted prompt pump-induced wavefront distortion of $5.5 \pm 1$ waves peak-tovalley $(\mathrm{P}-\mathrm{V})$ is twice as great as its maximum 2.7 -wave requirement. On the other hand, the predicted slab thermal distortion of $0.4 \pm 0.25$ waves is less than one-third its maximum 2.2-wave requirement. The predicted prompt and slab thermal distortions combined (in the worst case, $5.5+0.4=5.9$ waves) is $\sim 20 \%$ greater than the sum of the prompt and slab thermal requirements $(2.7+2.2=4.9$ waves $)$. The NIF's wavefrontcorrection system appears to have sufficient margin to correct for this modest increase in overall distortion. We therefore plan to submit an Engineering Change Request to rebalance the requirements for prompt and slab thermal distortions to be consistent with our performance predictions. 
Table E.1. Summary of NIF optical performance requirements and performance predictions.

\begin{tabular}{|c|c|c|c|c|c|c|}
\hline Paramoter & Requirement & Prodiction & Basts & Implications & Caveats & Recommendation \\
\hline $\begin{array}{l}\text { Average gain } \\
\text { coefficlent < }<>\end{array}$ & $>6(\% / \mathrm{cm})$ & $6.2 \pm 0.2(\% / \mathrm{cm})$ & $\begin{array}{l}\text { 3D ray-trace code } \\
\text { valldated by } \\
\text { AMPLAB } \\
\text { measurements }\end{array}$ & Requirement met & $\begin{array}{l}\text { Modeling was } \\
\text { performed using } \\
\text { AMPLAB's pump } \\
\text { carlty, rather than } \\
\text { NIF's }\end{array}$ & $\begin{array}{l}\text { Model performance using } \\
\text { NIF pump cavity design (now } \\
\text { underway). }\end{array}$ \\
\hline $\begin{array}{l}\text { Gain uniformity } \\
\text { parameter } \\
\alpha_{\text {pees }}<<\infty>\end{array}$ & $<1.05$ & $1.08 \pm 0.02$ & $\begin{array}{l}\text { 3D ray-trace code } \\
\text { validated by } \\
\text { AMPLAB } \\
\text { measurements }\end{array}$ & $\begin{array}{l}\text { With larger gain varlations, the input fluence } \\
\text { distribution must be tailored more strongly to } \\
\text { produce a uniform output fluence distribution. For } \\
\text { example, in the limkt of low-output fluence, the } \\
\text { input fluence distribution must have a peak-to- } \\
\text { valley (P-V) ratio of 8:1 to produce unform output } \\
\text { fluence. }\end{array}$ & $\begin{array}{l}\text { Modeling was } \\
\text { performed using } \\
\text { AMPLAB's pump } \\
\text { cavity, rather than } \\
\text { NAf's }\end{array}$ & $\begin{array}{l}\text { Use ray-trace and } \\
\text { propagation modeling to } \\
\text { evaluate gain vs gain } \\
\text { uniformity tradeoff as lamp } \\
\text { energy is reduced. }\end{array}$ \\
\hline & & & & & & \\
\hline
\end{tabular}


Table E.1 (cont.). Summary of NIF optical performance requirements and performance predictions.

\begin{tabular}{|c|c|c|c|c|c|c|}
\hline Parameter & Requirement & Prediction & Basis & Implications & Caveats & Recommendation \\
\hline $\begin{array}{l}\text { Prompt pump- } \\
\text { induced wavefront } \\
\text { dlstortion }\end{array}$ & $\begin{array}{l}<2.7 \text { waves P-V } \\
\text { of low-order } \\
\text { distortion }\end{array}$ & $5.5 \pm 1.0$ waves $P-V$ & $\begin{array}{l}\text { Prompt pump- } \\
\text { induced model } \\
\text { calibrated to } \\
\text { AMPLAB } \\
\text { measurements }\end{array}$ & See impllcations for slab thermal distortion below & $\begin{array}{l}\text { It is concelvable that } \\
\text { AMPLAB } \\
\text { measurements were } \\
\text { affected by flashlamp } \\
\text { pumping of } \\
\text { dlagnostic mirrors }\end{array}$ & $\begin{array}{l}\text { Improve prompt pump- } \\
\text { Induced model by using 3D } \\
\text { code and adding UV and IR } \\
\text { sources. Model AMPLAB } \\
\text { miror distortions. Use } \\
\text { model to evaluate feasiblity } \\
\text { of reducing prompt } \\
\text { wavefront distortlon by } \\
\text { using adusted lamp energles }\end{array}$ \\
\hline $\begin{array}{l}\text { Prompt pump- } \\
\text { induced steering } \\
\text { of beam centrold }\end{array}$ & $<8 \mu \mathrm{rad}$ & $4 \mu \mathrm{rad}$ & $\begin{array}{l}\text { Prompt pump- } \\
\text { induced model } \\
\text { callibrated to } \\
\text { AMPLAB } \\
\text { measurements }\end{array}$ & Requirement met & $\begin{array}{l}\text { It is concelvable that } \\
\text { AMPLAB } \\
\text { measurements were } \\
\text { affected by flashlamp } \\
\text { pumping of } \\
\text { diagnostic mirrors }\end{array}$ & $\begin{array}{l}\text { Evaluate with improved } \\
\text { model for prompt distortion }\end{array}$ \\
\hline \begin{tabular}{|l|} 
Shot-to-shot \\
variation in \\
prompt pump- \\
Induced wavefront \\
distortion
\end{tabular} & $<10 \%$ & $<10 \%$ & $\begin{array}{l}\text { Shot-to-shot } \\
\text { varlations } \\
\text { measured on } \\
\text { diamond and X } \\
\text { configurations in } \\
\text { AMPLAB give } \\
\text { upper limit }\end{array}$ & Requirement met & $\begin{array}{l}\text { Shot-to-shot energy } \\
\text { variations for } \\
\text { flashlamps could be } \\
\text { greater on NIF due to } \\
\text { parallel lamp circuit }\end{array}$ & $\begin{array}{l}\text { Modify 3D code to allow } \\
\text { lamp energies to be adjusted } \\
\text { individually. Model prompt } \\
\text { wavefront distortion with } \\
\text { random shot-to-shot lamp } \\
\text { energy variations, averaging } \\
\text { results over entire } \\
\text { multipassed beamiline }\end{array}$ \\
\hline $\begin{array}{l}\text { Pump-induced } \\
\text { depolarization }\end{array}$ & $\begin{array}{l}<0.05 \% \text { per } \\
\text { slab, aperture } \\
\text { averaged }\end{array}$ & $<0.01 \%$ & $\begin{array}{l}\text { Prompt pump- } \\
\text { induced model }\end{array}$ & Requirement met & None & None \\
\hline
\end{tabular}

4

1

1

J


Table E.1 (cont.). Summary of NIF optical performance requirements and performance predictions.

\begin{tabular}{|c|c|c|c|c|c|c|}
\hline Parameter & Requirement & Predlction & Basis & Implications & Caveats & Recommendation \\
\hline $\begin{array}{l}\text { Slab thermal } \\
\text { distortion }\end{array}$ & $\begin{array}{l}<2.2 \text { waves P-V } \\
\text { of low-order } \\
\text { distortion less } \\
\text { than } 7 \text { hours } \\
\text { aftor a shot (not } \\
\text { to preclude } 3 \\
\text { hours) }\end{array}$ & $\begin{array}{l}0.4 \pm 0.25 \text { waves after } \\
7 \text { hours; } \\
1.5 \pm 0.7 \text { waves after } \\
3 \text { hours }\end{array}$ & $\begin{array}{l}\text { Modeling gives } \\
\text { lower bounds, } \\
\text { AmPLAB } \\
\text { measurements } \\
\text { give upper bounds }\end{array}$ & $\begin{array}{l}\text { After } 7 \text { hours, predicted slab thermal dlstortion ls } \\
<30 \% \text { of max allowed value and <15\% as large as } \\
\text { predicted prompt pump-induced wavefront } \\
\text { distortion. Sum of predicted prompt + thermal } \\
\text { distortion is } 5.9 \pm 1.0 \text { waves, } 0-40 \% \text { greater than } \\
\text { the sum of the prompt + thermat max limits }(2.2+ \\
2.7=4.9 \text { waves). It appears this increase can be } \\
\text { accomodated within the margin of the NIF } \\
\text { deformable mirror. }\end{array}$ & $\begin{array}{l}\text { Qualltatlve agreement } \\
\text { between modeling } \\
\text { and measurements } \\
\text { was not achieved }\end{array}$ & $\begin{array}{l}\text { Adjust requirements to } \\
\text { predictions, after prompt } \\
\text { pump-induced distortion } \\
\text { action items recommeded } \\
\text { above have been carried out }\end{array}$ \\
\hline $\begin{array}{l}\text { Thermal steering } \\
\text { of beam centroid }\end{array}$ & $<8 \mu \mathrm{rad}$ & None & Not applicable & Low risk & None & $\begin{array}{l}\text { Evaluate experimentally on } \\
\text { first bundle }\end{array}$ \\
\hline $\begin{array}{l}\text { Slab thermal } \\
\text { dlstortion drift } \\
\text { one hour prior to } \\
\text { shot }\end{array}$ & $<10 \%$ & $\begin{array}{l}-10 \% \text { (of max } \\
\text { allowed slab } \\
\text { distortion) between } 7 \\
\text { and } 8 \text { hours after } \\
\text { shot, } \sim 20 \% \text { between } \\
3 \text { and } 4 \text { hours after } \\
\text { shot }\end{array}$ & $\begin{array}{l}\text { AMLAB } \\
\text { measurements }\end{array}$ & Requirement met & None & Verity on first bundle \\
\hline $\begin{array}{l}\text { Gas thermal } \\
\text { distortions }\end{array}$ & $\begin{array}{l}<5 \text { rad added } \\
\text { beam } \\
\text { divergence } 7 \\
\text { hours after } \\
\text { shot, not to } \\
\text { preclude } 3 \\
\text { hours after shot }\end{array}$ & $\begin{array}{l}0.9 \pm 0.4 \mu \text { rad added } \\
\text { beam divergence } 7 \\
\text { hours after shot; } 3.3 \\
\pm 1.7 \mu \text { rad added } \\
\text { beam divergence } 3 \\
\text { hours after shot }\end{array}$ & $\begin{array}{l}\text { AMPLAB } \\
\text { wavefront and } \\
\text { temperature } \\
\text { measurements, } \\
\text { thermal modeling, } \\
\text { and propagatlon } \\
\text { modeling. Cooling } \\
\text { with gas } 1^{\circ} \mathrm{C} \text { below } \\
\text { amblent. }\end{array}$ & Requirement met & $\begin{array}{l}\text { Gas distortions not } \\
\text { measured using } \\
\text { chilled-gas cooling in } \\
\text { AMPLAB }\end{array}$ & $\begin{array}{l}\text { Verify focusability } \\
\text { requirements on first bundie }\end{array}$ \\
\hline
\end{tabular}


Table E.1 (cont.). Summary of NIF optical performance requirements and performance predictions.

\begin{tabular}{|l|l|c|c|c|c|c|}
\hline \multicolumn{1}{|c|}{ Parameter } & Requirement & Prediction & Basis & Implications & Caveats & Recommendation \\
\hline $\begin{array}{l}\text { Mounting-stress- } \\
\text { induced wavefront } \\
\text { distortion }\end{array}$ & $\begin{array}{l}<0.1 \text { waves per } \\
\text { slab }\end{array}$ & None & Not applicable & $\begin{array}{l}\text { Norform wavefront } \\
\text { measurements using } \\
\text { prototype slab holder }\end{array}$ \\
\hline $\begin{array}{l}\text { Mounting-stress- } \\
\text { inducced } \\
\text { depolarization }\end{array}$ & $\begin{array}{l}<0.05 \% \text { per } \\
\text { slab, aperture } \\
\text { averaged }\end{array}$ & None & Not applicable & None & $\begin{array}{l}\text { Nerform depolarization } \\
\text { measurements using } \\
\text { prototype slab holder }\end{array}$ \\
\hline
\end{tabular}

1

1

1




\subsection{Optical Performance Requirements for the NIF Amplifiers}

The NIF amplifiers must provide sufficient gain and stored energy to meet requirements for laser energy and power while adding minimal wavefront distortion to the laser beams. Accordingly, there are formal requirements for aspects of amplifier optical performance: gain, gain uniformity, prompt pump-induced wavefront distortion, long-term thermal wavefront recovery, and depolarization. These requirements flow down from the NIF functional requirements for beam energy, power, focusability, power balance, pointing stability, and shot rate $[1.1,1.2,1.3]$. Propagation modeling has shown that the current amplifier performance requirements are consistent with the NIF laser meeting its performance requirements, provided other laser components perform as required [1.4].

\subsection{Formal requirements}

The current requirements for the NIF amplifiers are described in the NIF Subsystem Design Requirements, Rev D of section 3.2.1. [1.1] These requirements are:

- Average gain coefficient (3.2.1.1.1)-The average gain coefficient at line center shall be greater than $5 \% / \mathrm{cm}$. The design shall not preclude future implementation of multicolor operation at up to four separate $1 \omega$ wavelengths from $1.0523-1.0553$ microns.

- Gain uniformity (3.2.1.1.2)-The beamline peak-to-average ratio for the small signal-gain coefficient will be $<1.05$, in any aperture at the $20 \%$ explosion fraction (nominal) pump rate.

- Prompt Pump-Induced Wavefront Distortion (3.2.1.1.3)—Amplifier pumping processes shall not produce coherent pump-induced wavefront distortion that exceeds the following values in normal multipass operation:

$<0.0025$ waves/slab per pass variation in optical path length across each aperture (4 passes, peak-to-valley [P-V]) with a spatial frequency of $0.0-0.5$ cycles.

$<0.05$ waves/slab per pass variation in optical path length across each aperture (4 passes, $\mathrm{P}-\mathrm{V}$ ) with a spatial frequency of $0.5-1.5$ cycles.

$<0.0125$ waves/slab per pass variation in optical path length across each aperture (4 passes, $\mathrm{P}-\mathrm{V}$ ) with a spatial frequency of $1.5-12$ cycles.

Horizontal or vertical steering of the beam centroid caused by pump-induced distortions with a spatial frequency of less the 0.5 cycles shall be less than $8 \mu \mathrm{rad}$ for each aperture. 
The shot-to-shot variation of the pump-induced wavefront distortion shall not vary by more than $\pm 10 \%$.

- Recovery from Optical Distortion Due to Slab Residual Temperature Differences (3.2.1.1.5.1)-In less than 7 hours (not to preclude $<3$ hours) after each shot, the coherent wavefront distortion caused by a temperature difference in an amplifier slab shall not exceed the following limits:

$<0.0025$ waves/slab per pass variation in optical path length across each aperture ( 4 passes, $\mathrm{P}-\mathrm{V}$ ) with a spatial frequency of $0.0-0.5$ cycles.

$< \pm 0.04$ waves/slab per pass (P-V) variation in the optical path length across each aperture with a spatial frequency of $0.5-1.5$ cycles.

$<0.0125$ waves/slab per pass variation in optical path length across each aperture (4 passes, $P-V$ ) with a spatial frequency of $1.5-12$ cycles.

Horizontal or vertical steering of the beam centroid caused by slab distortions with a spatial frequency of less the 0.5 cycles shall be less than $8 \mu \mathrm{rad}$ for each aperture.

The wavefront distortions specified above shall not drift more than $\pm 10 \% / \mathrm{hr}$ for a period 1 hour prior to a shot.

- Recovery from Optical Distortion Due to Cavity Gas (3.2.1.1.5.2)—Optical distortion is caused by refractive index and temperature variations in the gas that is convectively heated by pump cavity parts after a shot. In less than seven hours after each shot, this uncorrectable beam divergence with spatial scale lengths of 2-20 cycles shall add less than 5 microradians to the full angle, enclosing $80 \%$ of the beam energy, $1.05 \mu \mathrm{m}$. The system design should not preclude achieving this requirement in less than three hours.

This additional uncorrectable beam divergence is the cumulative effect of divergence from both amplifier (main and power) in a beamline.

The beam divergence allocation is in addition to all other sources of beam divergence.

- Depolarization-Pump-Induced and Mounting-Stress-Induced (3.2.1.1.4)—The pump-induced depolarization shall be $<0.05 \%$ aperture averaged per slab.

The stress-induced depolarization shall be limited to $<0.05 \%$ aperture averaged per slab. 
- Wavefront Distortion Induced by Mounting Stresses (3.2.1.1.10)-The mounting of the slab in its holder shall introduce no more than \pm 0.1 wave of distortion. This allowance is independent of fabrication allowances for the laser slab.

The rate of change of the distortion shall be less than 0.2 waves $/ \mathrm{cm}$.

This distortion shall not vary more than $20 \%$ from shot to shot.

\subsection{Working requirements}

The formal requirements for wavefront distortion are complex, with specified limits over different spatial-frequency ranges. In practice, the NIF amplifier designers use simplified requirements that are approximately equivalent. These are:

1. The prompt pump-induced wavefront distortion produced by a multipassed NIF amplifier chain shall be less than 2.7 waves, peak-to-valley.

2. The slab thermal distortion produced by a multipassed NIF laser chain shall be less than 2.2 waves, peak-to-valley.

3. Both the prompt pump-induced distortion and slab thermal distortion shall be sufficiently low order so as to be largely correctable with the NIF deformable mirror system. 


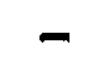




\section{NIF Amplifier Design}

The major laser component in the NIF will be the flashlamp-pumped Nd:glass amplifiers, which must provide sufficient gain and stored energy to meet requirements for laser energy and power while adding minimal wavefront distortion to the laser beams. The NIF amplifiers differ from those used in previous ICF laser systems mainly in their overall scale and packaging. Figure 2.1 shows a two-slab-long, $4 \times 2 \mathrm{NIF}$ amplifier module, where the $\mathrm{n} \times \mathrm{m}$ designation denotes the number (height $\mathrm{x}$ width) of parallel amplifying channels or beam apertures that are combined. Each $4 \times 2$ beam bundle uses an eleven-slab-long main amplifier that the beam passes four times, and a five-slab-long power amplifier that the beam passes twice. The NIF amplifiers use $40-\mathrm{cm}$-square apertures, which are near the practical size limit imposed by laser-slab fabrication techniques. Large aperture size reduces system costs by reducing the number of laser beams needed to produce the required energy on target. Previously, the largest amplifiers constructed were the $2 \times 2$ Beamlet amplifiers, which combined only four $40-\mathrm{cm}$-square apertures [2.1].

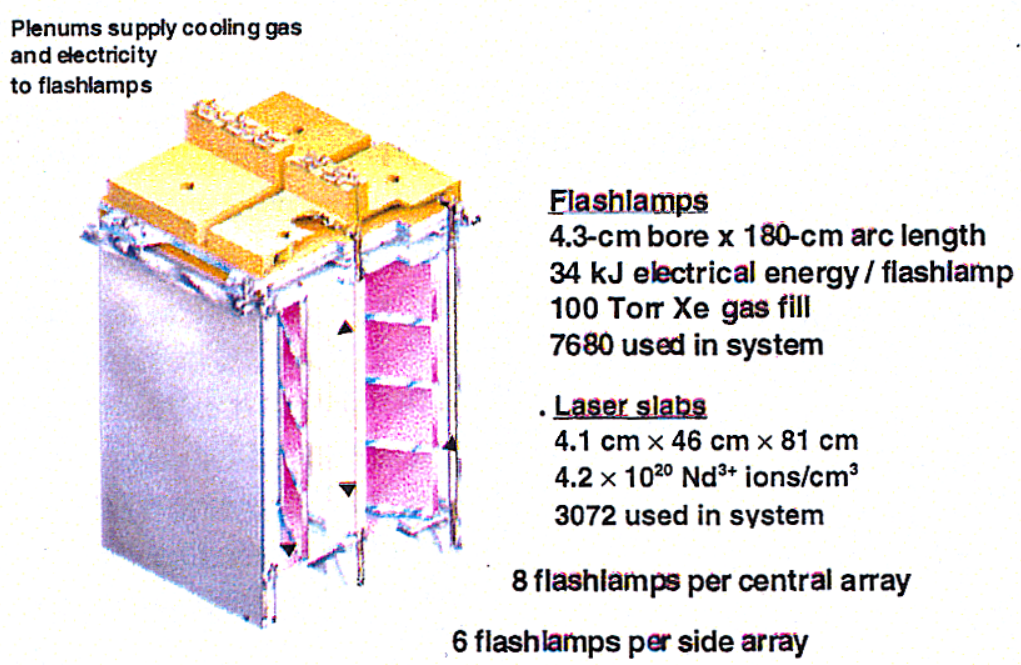

Figure 2.1. A two-slab-long, $4 \times 2$ NIF amplifier module.

Amplifiers with combined beams were first proposed by LLNL in 1978 as a way to reduce the cost of MJ-class fusion laser systems [2.2]. Combining beams in a single enclosure reduces costs in three ways: 1) by making amplifiers more compact, thereby reducing the size and cost of the building; 2 ) by increasing pumping efficiency, thereby reducing the size and cost of the power-conditioning system and enhancing the stored energy density; and 3) by reducing the total number of amplifier parts. The NIF design achieves considerable cost savings by making the NIF amplifiers larger than the Beamlet amplifiers. Similar amplifiers will be used in the Laser Megajoule (LMJ), a 240-beam laser system now being developed and designed by the French Commissariat a l'Energie Atomique (CEA). 


\subsection{Pump cavity}

The NIF amplifiers provide optical gain at the $1.053-\mu \mathrm{m}$ wavelength by using neodymium-doped, phosphate glass, rectangular laser slabs oriented at Brewster's angle with respect to the beam, to eliminate reflection losses. The slabs have absorbing glass edge claddings to prevent internal parasitic laser oscillation. Each slab holder supports four slabs, one stacked above the other. The central flashlamp cassettes pump slabs in both directions, while the side flashlamp cassettes with large silver reflectors pump slabs in one direction. Glass blastshields, placed between the flashlamps and the laser slabs, serve three purposes: (1) they prevent acoustic waves generated by the flashlamps from propagating into the beam path and causing wavefront distortion, (2) they provide a contamination barrier between the flashlamp cavity and the critical slab cavity, and (3) they form one wall of the channel used for flowing cooling gas around the flashlamps. Figure 2.2 shows a plan view of a NIF amplifier slab column illustrating the arrangement of the slabs, flashlamps, blastshields, and reflectors.

The NIF amplifier design is characterized by a number of important features. The $4.3-\mathrm{cm}$ bore $\times 180-\mathrm{cm}$ arc length flashlamps are energized by an electrical pulse with a duration of $360 \mu \mathrm{s}$. The neodymium ions in the laser slabs are optically excited by the flashlamp light to produce a peak gain coefficient of about $5.0 \% / \mathrm{cm}$, averaged through all slabs in the chain. The transverse gain uniformity is determined by the distribution of flashlamp light across the slab and by the effect of amplified spontaneous emission (ASE). In large slabs such as the NIF's, which measure $4.1 \mathrm{~cm} \times 45.8 \mathrm{~cm} \times 80.9 \mathrm{~cm}$, ASE preferentially depletes the gain near the slab's ends because this position has the longest path length for internal amplification $[2.1,2.3,2.4]$. The peak-to-average ratio for the gain coefficient, evaluated across the chain aperture, is $\approx 1.08$. Pump-induced wavefront distortion can occur from disk bending produced by prompt heating of the laser slab by flashlamp light, which is imbalanced from one side of the slab to the other. The total prompt pump-induced wavefront distortion produced by the entire amplifier chain is specified to be less than six waves, so that the distortions can be corrected with a deformable mirror provided in each NIF laser chain. 


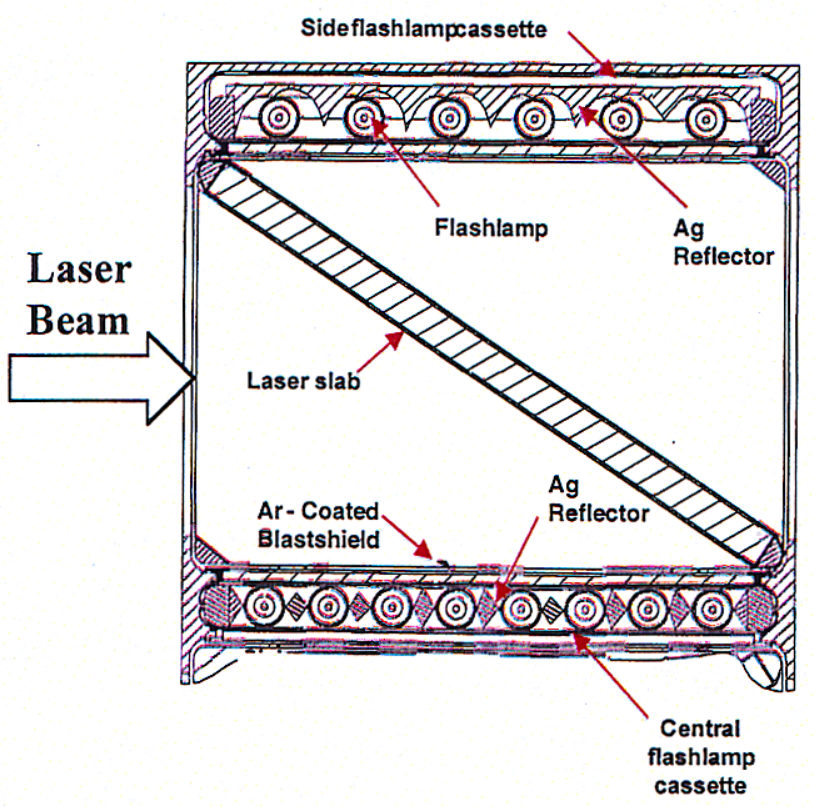

Figure 2.2. Plan view of the NIF amplifier pump cavity.

The NIF amplifiers use several features to increase efficiency. The side flashlamp arrays use silver reflectors with involute-shaped reflectors, designed to reflect flashlamp light toward the laser slabs while returning little flashlamp light back to the absorbing flashlamp plasma. Compared with flat reflectors, the involute-shaped reflectors reduce the flashlamp electrical energy required to meet the gain requirement by $\sim 12 \%$, overall [2.5]. Additional reductions in flashlamp electrical energy are achieved by using sol-gel AR coatings on both sides of the blastshields $(\sim 10 \%)$ and by preionizing the flashlamps with weak electrical pulses delivered several hundred microseconds before the main pulse $(\sim 10 \%)$. Preionization causes the flashlamp arc to develop more uniformly and increases the electrical-to-optical conversion efficiency of the flashlamp plasma. Overall, the predicted storage efficiency of the NIF amplifiers is $3.8 \%$, which is significantly higher than in previous ICF lasers $(3.0 \%$ and $1.8 \%$ for the Beamlet and Nova amplifiers, respectively) [2.1]. Storage efficiency is defined as the total extractable energy stored in the laser slabs divided by the electrical energy delivered to the flashlamps. The NIF pump cavity design also uses skewed diamond-shaped reflectors in the central flashlamp arrays, which improve gain uniformity by directing flashlamp light to specified regions of the laser slabs.

\subsection{Thermal management}

Waste pump heat causes residual thermal wavefront distortions that persist for many hours after each shot. A principal cause of the distortions is thermal gradients in the laser slabs set up by differences between the heating and cooling rates of the laser slab and its edge cladding. Additional distortion is caused by convection currents set up by temperature differences between laser slabs, blastshields, and reflectors. The slab distortions tend to be slowly varying, low-order distortions that are largely correctable with a deformable mirror. Gas distortions, however, have high spatial frequencies and are more rapidly varying, making them difficult to correct. 
To accelerate the thermal recovery rate, the NIF flashlamps will be actively cooled by flowing gas. Flashlamp cooling is effective since some $60 \%$ of the pump waste heat resides in the flashlamp envelopes immediately after a shot. Although water cooling has been successfully used to accelerate the shot rate of the Omega Laser at the University of Rochester [2.6], gas cooling was chosen for the NIF to eliminate water-jacket tubes and to leave more room for efficiency-enhancing reflectors. The NIF flashlamp cooling system will provide gas flow rates of up to 20 cubic feet per minute per flashlamp, with the gas flow direction alternating between flashlamp cassettes as shown in Figure 2.3. The inlet temperature of the cooling gas will be controlled over a $\pm 5^{\circ} \mathrm{C}$ range centered about the ambient temperature with $\pm 0.3^{\circ} \mathrm{C}$ accuracy.

The thermal wavefront distortion in the laser slabs is specified to be less than 2.2 waves, and the gas distortions are specified to add less than $5 \mu \mathrm{rad}$ to the full beam divergence. Flowing room-temperature gas over the flashlamps is expected to achieve these conditions in less than seven hours after each shot. With a one-hour period set aside for beam realignment after the amplifiers have thermally recovered, we expect to achieve an eight-hour period between laser shots. More rapid thermal recovery of the amplifiers appears feasible by slightly chilling the flashlamp cooling gas, however.

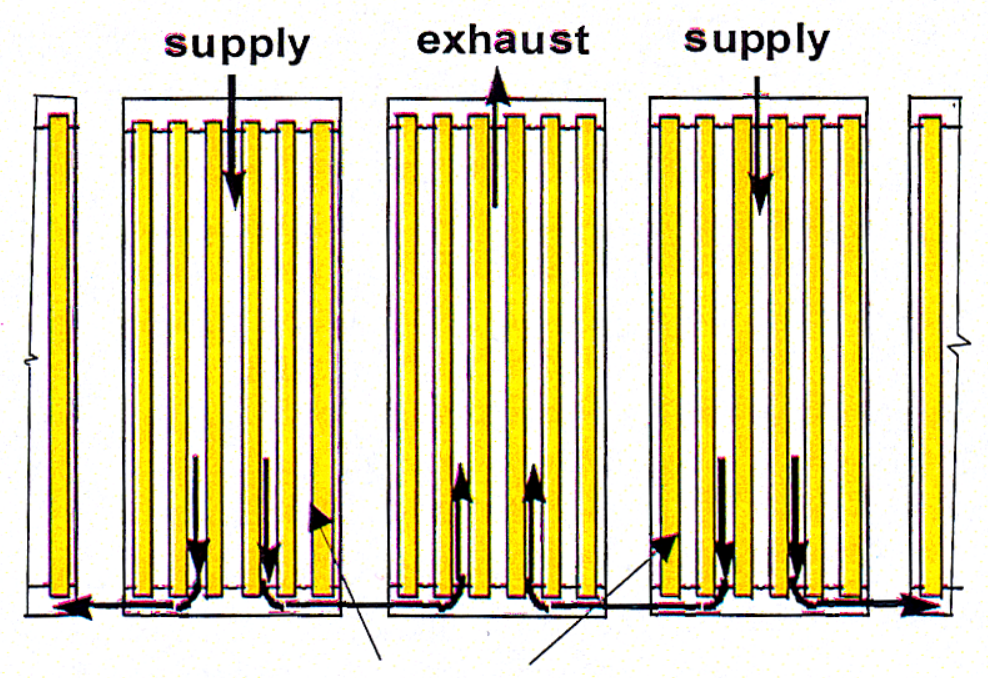

Flashlamps

Figure 2.3. By alternating the direction of cooling-gas flow through the flashlamp cassettes, coolinggas connections can be made at the top of the amplifier, which simplifies the amplifier mechanical design. 


\subsection{Mechanical design}

The large scale of the NIF amplifiers requires new mechanical designs that allow convenient assembly and maintenance. Accordingly, the NIF amplifiers use a modular design in which the most critical components, including the flashlamps, laser slabs, and reflectors, are mounted in line-replaceable units or cassettes that can be readily inserted or removed without disturbing their neighbors [2.7] (see Figure 2.4).

Installation and removal of flashlamp cassettes and four-high slab cassettes are accomplished by using sealed maintenance carts that access the bottom of the amplifiers. The cassettes are inserted and removed from their enclosure, called the frame assembly unit, which is supported by top plates mounted to an overhead support structure. Plenums distribute electricity and cooling gas to the flashlamps from the top through holes in the top plate.

Assembly of these amplifiers begins in an off-line cleanroom, where the frame assembly units are cleaned and the top plates and blastshields with AR coatings are installed. A flashlamp-light-resistant polymer is used to bond the blastshields to a metal frame, and silicone inflatable seals are used to seal the metal frame to the inside of the frame assembly unit. These seals reduce leak rates between the flashlamp cavity and the slab cavity. After the blastshields have been installed, frame assembly units are bolted together to form five- and eleven-slab-long units that are transported to the laser bay where they are mounted to an overhead support structure by their top plates.

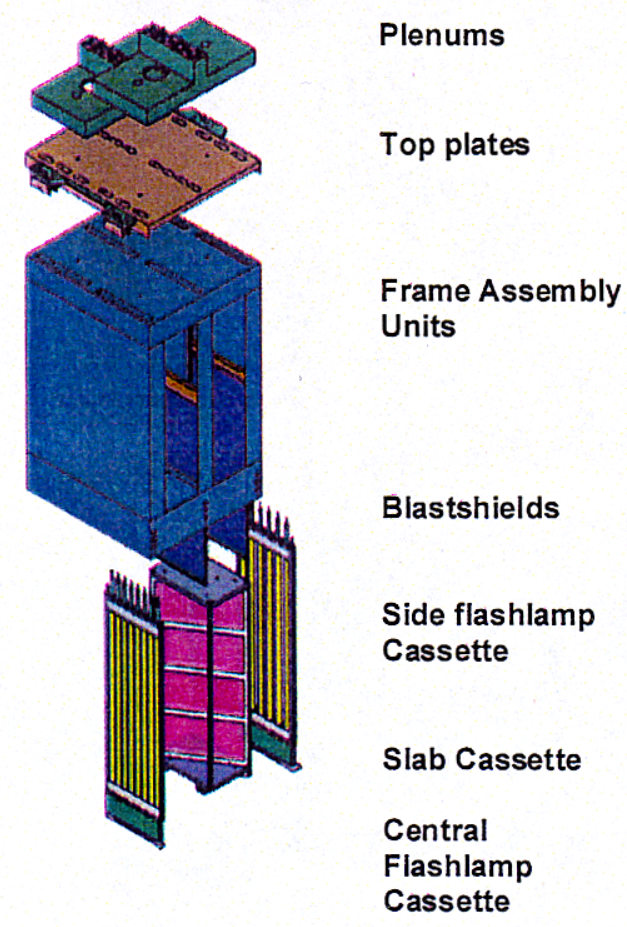

Figure 2.4. The NIF amplifiers use a modular design. 
The laser slabs, reflectors, and metal parts that comprise the slab cassettes are spraycleaned and assembled in an elevated, class-100 clean room. High cleanliness levels must be maintained, since small particles resting on the laser slabs cause damage when heated by flashlamp and laser light. Current specifications call for the laser slabs and metal surfaces to be maintained at cleanliness levels of 50 and 100, respectively, which correspond to particle-size distributions in which there are only one 50- or 100-micron particle per square foot of surface area, respectively [2.8]. Following assembly, the slab cassettes are lowered into a specialized clean cart. Once the cart has been moved to the laser bay, it docks to the bottom of a frame assembly unit and establishes a hermetic seal to maintain cleanliness. After the top cover of the cart and the bottom cover of the frame assembly unit have been pressed together to trap residual particles, the cover pair is moved to the side to open a passageway for the slab cassette to be raised into the frame assembly unit. Rollers mounted in the corners of the cassette guide the cassette during insertion and prevent metal-on-metal rubbing, which would generate particles. A fail-safe mechanism in the cart activates latches that hold the slab cassette in place. Slab cassettes can be removed for occasional refurbishment by reversing this installation process. Figure 2.5 shows a prototype slab cassette cart, which has successfully completed some 50 slab-cassette transfers in our laboratory. Similar carts will be used to install and remove the NIF flashlamp cassettes and blastshields.

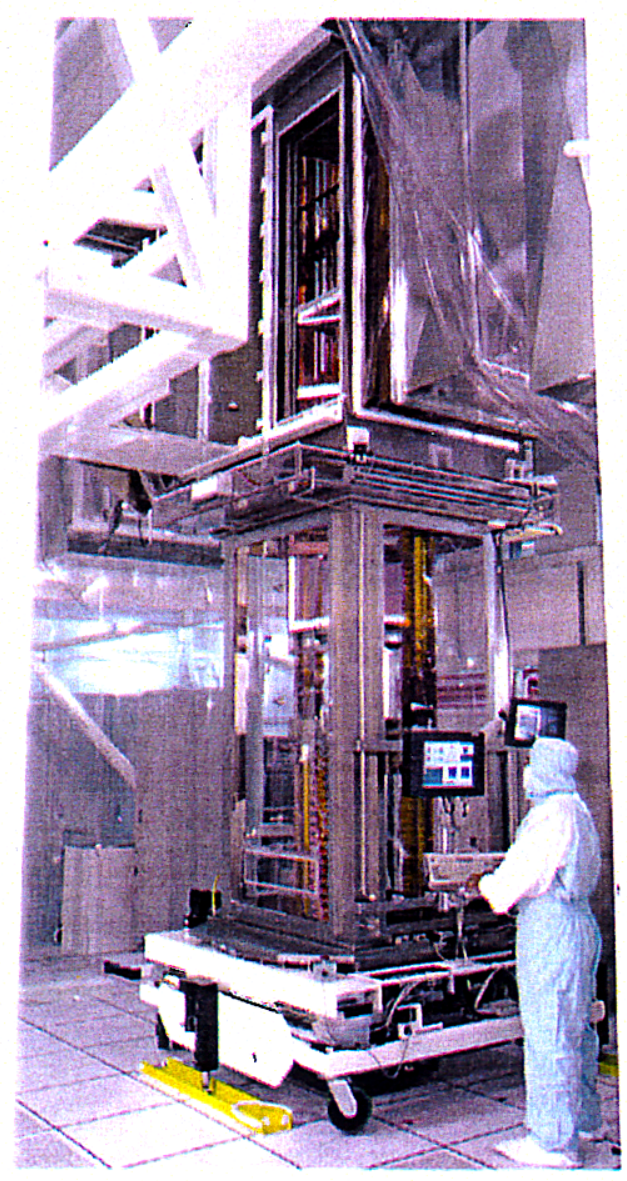

Figure 2.5. Our prototype slab cassette cart shown inserting a slab cassette into the NIF prototype amplifier. 


\section{Description of NIF Prototype Amplifier and Experiments}

\subsection{Description of prototype amplifier and comparison with the NIF Title II baseline amplifier design}

This section describes the NIF prototype amplifier that was built and tested in the Amplifier Module Prototype Laboratory (AMPLAB) at LLNL during 1997-98. Because the NIF design team used the prototype amplifier as the starting point for the NIF Title II design described in the previous section, the two designs are very similar. Consequently, this section emphasizes comparisons between the two designs while providing additional details about the prototype.

Figure 3.1 shows plan views for the three-slab-long sections the NIF prototype amplifier (AMPLAB) and the NIF Title II baseline design. Despite their similar appearances, the two designs have slight differences in component dimensions and relative positions, arising from decisions to improve the Title II design relative to the prototype. The most significant differences are described below.

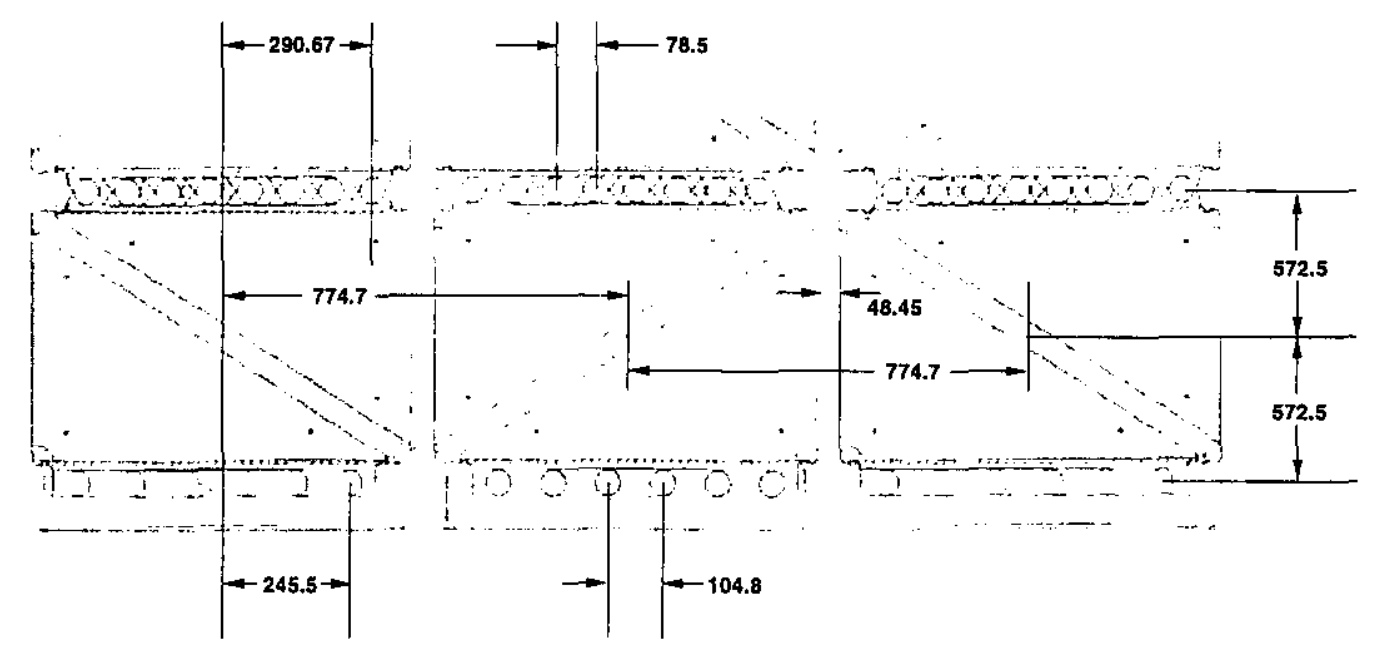

(a) NIF prototype amplifier (AMPLAB)

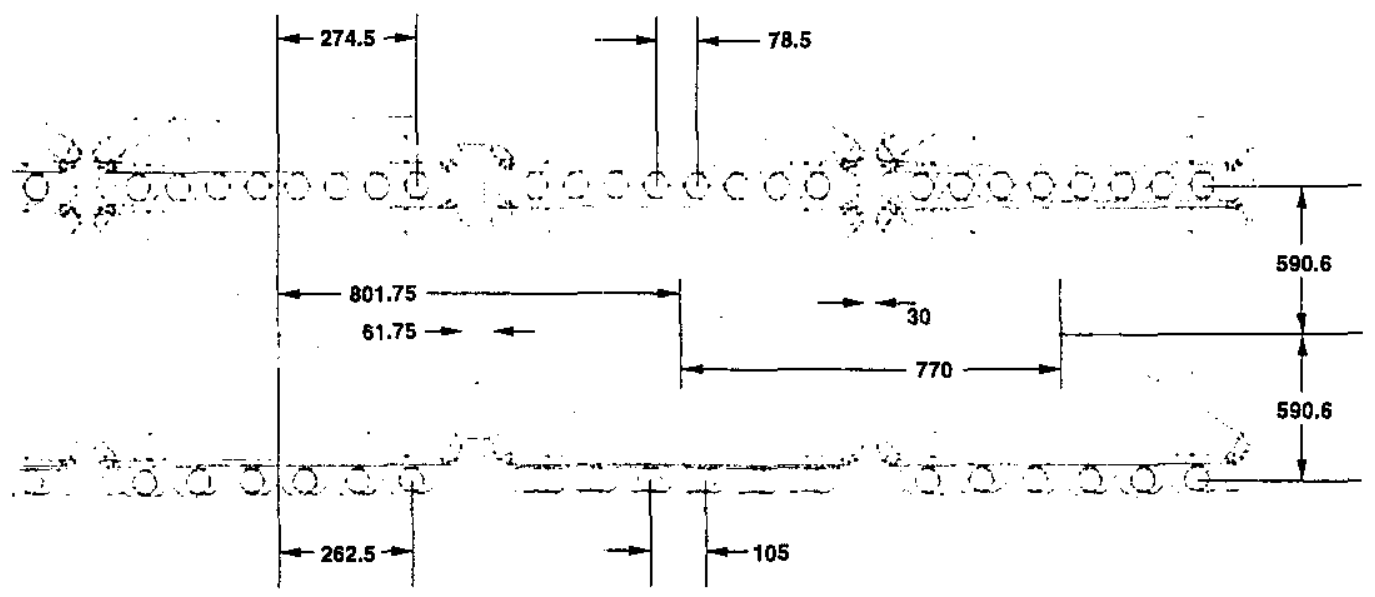

(b) NIF Title II baseline design

Figure 3.1. Plan views for three-slab-long sections of (a) the NIF prototype amplifier (AMPLAB) and (b) the NIF Title II baseline amplifier design. Dimensions are in $\mathbf{m m}$. 
Overall, the NIF Title II design is less compact than the NIF prototype amplifier. The Title II design uses $5-\mathrm{mm}$ insertion clearances between the slab cassettes and the frame assembly units, while the prototype amplifier used 3-mm insertion clearances. Larger insertion clearances reduce the risk of the slab cassette rubbing against the frame assembly unit during insertion and allow fabrication tolerances to be relaxed to reduce costs. The Title II design also uses 10 -mm-thick blastshields, while the prototype uses 6-mm-thick blastshields. Thicker blastshields are used in the Title II design to reduce fracture risk, as the blastshields must hold off a pressure difference between the slab cavity and the flashlamp cavity when turbulent gas is used to cool the flashlamps. The Title II design uses two- and three-slab-long frame assembly units, while the prototype design uses only one-slab-long frame assembly units. Consequently, the separation between slab centers in the direction of the beam alternates between $801.75 \mathrm{~cm}$ and 770 $\mathrm{cm}$ in the Title II design, while this separation was fixed at $774.7 \mathrm{~cm}$ in the prototype amplifier. Relative to the prototype amplifier, the Title II design has a $1.8-\mathrm{cm}$ greater average separation between the flashlamps and the laser-slab center in the horizontal direction perpendicular to the beam, and a $1.1-\mathrm{cm}$ greater average separation between slabs in the direction parallel to the beam.

In the Title II design, flat silver reflectors that are coplanar with the large triangular reflectors in the top and bottom of the pump cavity are installed in the gaps between adjacent frame assembly units. The prototype amplifier had no such reflectors, and presumably most of the light falling in the gaps was lost after falling on low-reflectance aluminum or getting trapped under the large triangular reflectors. The Title II design also has similar although smaller gaps between slab cassettes within each frame assembly unit that are not currently covered by reflectors, however. Overall, the total area of the holes and slots through which light is lost is $4300 \mathrm{~cm}^{2}$ in the Title II design, compared with $3720 \mathrm{~cm}^{2}$ in the prototype amplifier. Gain modeling treating the pump cavity as a hohlraum shows this difference in total hole area and gives the Title II design a $\sim 1.6 \%$ greater average gain coefficient than the prototype amplifier, other factors being equal [3.1].

In the Title II design, the flashlamps are centered in the central and side flashlamp arrays. In the prototype amplifier, the flashlamps are offset in the direction of the beam by $\sim 1 \mathrm{~cm}$. Centering the flashlamps eliminates the need to design and manufacture two mirror-image variants of side flashlamp cassettes and top-hat plenums, allowing one design to be used for each of these parts. The top-hat plenums are affected because they provide the quick-disconnect high-voltage connections to the flashlamp cassettes. Raytrace modeling shows the $1-\mathrm{cm}$ shift causes only slight effects on gain and gain uniformity [3.2].

Whereas the slab masks in the prototype amplifier were $\sim 1 / 16$-in.thick textured stainless steel, the slab masks in the Title II design are 1-cm-thick aluminum with tapering to reduce slab shadowing (see Figure 3.2). The purpose of the thicker masks in the Title II design is to reduce the risk of point-loading the corners of the slabs, a problem that became apparent during the prototype-amplifier tests. The Title II masks are bare, machined aluminum, while the prototype amplifier masks were silvered. Three- 
dimensional (3D) ray-trace code calculations are planned to determine the effect of the mask differences on optical performance.

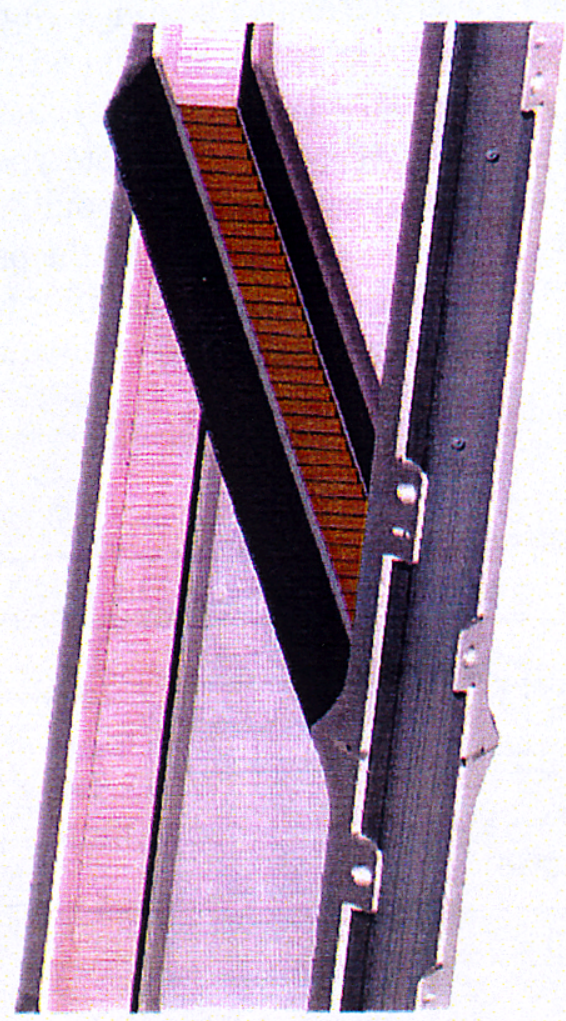

Figure 3.2. The slab masks in the NIF baseline design are $\sim 1 \mathrm{~cm}$ thick, with triangular cross sections for the top, bottom, and sides to reduce the shadowing of flashlamp light by the masks. The baseline design reduces costs by using masks of bare aluminum without silver.

The largest and most important reflectors in the amplifiers are the reflectors in the flashlamp cassettes and the triangular-shaped reflectors in the top and bottom of the slab cavity. The Title II design uses protectively coated silver for these reflectors, while the prototype amplifier used bare electrochemically deposited silver.

The least important reflectors in the amplifiers are the reflectors that have a small surface area and which reflect only a small fraction of the pump light arriving at the laser slabs. All such reflectors used electrochemically deposited silver in the prototype amplifier. However, the Title II design uses lower-reflectance surfaces to reduce costs. For example, the "clamshell" reflectors at the top and bottom of the flashlamp cassettes use Everbrite 88, an anodized aluminum surface that has a stable average reflectance of about $80 \%$, averaged over the 400 - to 1000 -mm neodymium pump bands. Also, as described above, the slab masks have uncoated, machined-aluminum surfaces. Modeling is planned to determine the effect of these cost-saving compromises.

Reflectance measurements performed on reflectors removed from the prototype amplifier showed evidence of significant tarnishing. Measurements made near normal incidence at $670 \mathrm{~nm}$ with a hand-held reflectometer showed $89-93 \%$ reflectance, compared with 96-97\% reflectance for fresh electrochemically-deposited silver. 
The Title II design uses Schott B-270 blastshields with improved, two-layer, sol-gel antireflective coatings, while the prototype amplifier used Pilkington SW float glass blastshields with single-layer sol-gel antireflective coatings. Figure 3.3 shows transmission measurements made on small samples that are representative of the blastshields used in the Title II and prototype amplifier designs. Initially, the average transmission of the Title-II-type sample was about $3 \%$ greater than the prototype amplifier-type sample. However, after 5000 shots, the Title-II-type sample had about 3\% lower average transmission than the prototype-amplifier-type sample. As the prototype amplifier blastshields received fewer than 500 shots during the amplifier testing, it seems likely that the blastshield transmission remained close to the initial values throughout the tests.

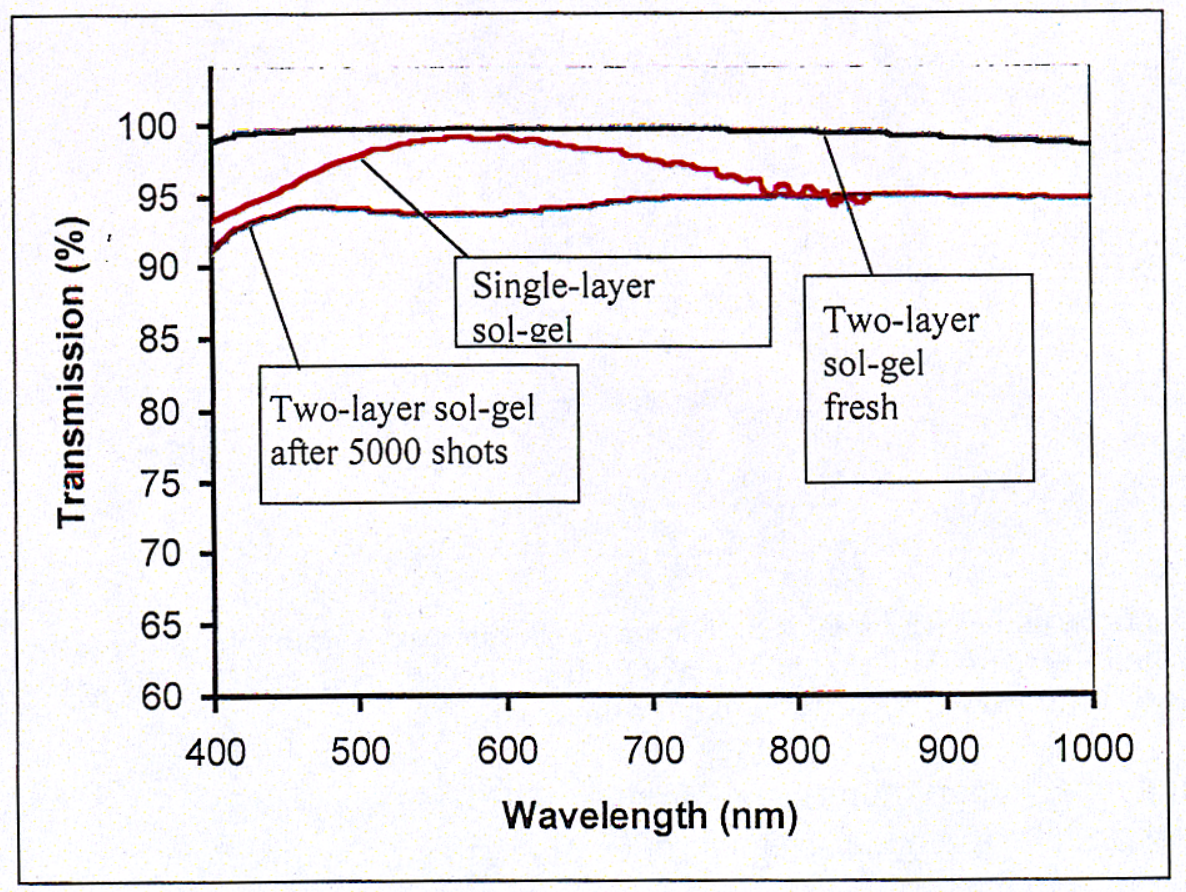

Figure 3.3. Measured normal-incidence transmission for a single-layer sol-gel, AR coating on 6-mmthick SW float glass, representative of the NIF prototype amplifier blastshields; and a two-layer solgel, AR coating on 10-mm-thick B-270 glass, representative of the NIF baseline design. Exposure to 5000 flashlamp shots reduced the average transmission of the latter by about $6 \%$. Measurement uncertainties are $\sim \pm 1 \%$.

Both the Title II and the prototype amplifier designs use diamond-shaped reflectors between the flashlamps in the central array to reduce lamp-to-lamp transfer and to increase pumping efficiency. The diamond-shaped-reflector surfaces were constructed from chords connecting points on an ellipse, with foci at the centerline of the flashlamp and a target point on the slab. Modeling shows that replacing sections of the ellipse with straight chords to reduce fabrication costs has only a small effect on the pump distribution [3.3].

Both the Title II and prototype amplifier designs use side-array reflectors with surfaces defined by both ellipses and involutes. The regions of the reflector that had direct line of sight to specific target points on the slab were ellipses, constructed using the 
centerline of the flashlamp and a target point on the slab as foci. The regions of the sidearray reflector without a direct line of sight to the target point were involutes, which improve pumping efficiency by reflecting flashlamp light in the general direction of the slab without passing through the absorbing flashlamp plasma. Figures 5.21 and 5.22, in the section on Prompt Pump-Induced Wavefront Measurements and Modeling, show plan views of the prototype and Title II designs, respectively, with lines drawn indicating locations of target points on the slabs.

The Title II and prototype amplifier designs used similar slab dimensions and pumped-area dimensions, as shown in Table 3.1.

Table 3.1. Title II and prototype amplifier slab and pumped-area dimensions.

\begin{tabular}{|c|c|c|c|c|c|}
\hline & \multicolumn{3}{|c|}{ Slab dimensions } & \multicolumn{2}{|c|}{$\begin{array}{l}\text { Pumped-area } \\
\text { dimensions }\end{array}$} \\
\hline & $\begin{array}{l}\begin{array}{l}\text { length } \\
(\mathrm{mm})\end{array} \\
\end{array}$ & \begin{tabular}{|l}
$\begin{array}{l}\text { width } \\
(\mathrm{mm})\end{array}$ \\
\end{tabular} & \begin{tabular}{|l|}
$\begin{array}{l}\text { thickness } \\
(\mathrm{mm})\end{array}$ \\
\end{tabular} & \begin{tabular}{|l}
$\begin{array}{l}\text { length } \\
(\mathrm{mm})\end{array}$ \\
\end{tabular} & \begin{tabular}{|l|} 
width \\
$(\mathrm{mm})$
\end{tabular} \\
\hline $\begin{array}{l}\text { NIF prototype } \\
\text { amplifier (LLNL } \\
\text { slabs) }\end{array}$ & $\begin{array}{l}808.85 \\
\pm 0.5 \\
\end{array}$ & $\begin{array}{l}457.47 \\
\pm 0.5 \\
\end{array}$ & $\begin{array}{l}40.0+0.0 / \\
-1.0\end{array}$ & $\begin{array}{r}773.11 \\
\pm 0.25 \\
\end{array}$ & $400 \pm 0.25$ \\
\hline $\begin{array}{l}\text { NIF prototype } \\
\text { amplifier (CEA } \\
\text { slabs) }\end{array}$ & $\begin{array}{l}808.00 \\
\pm 0.5 \\
\end{array}$ & $\begin{array}{l}458.00 \\
\pm 0.5 \\
\end{array}$ & $40.0 \pm 0.2$ & $\begin{array}{r}773.11 \\
\pm 0.25 \\
\end{array}$ & $400 \pm 0.25$ \\
\hline $\begin{array}{l}\text { NIF baseline } \\
\text { design }\end{array}$ & $\begin{array}{l}808.50 \\
+0 /-1.0 \\
\end{array}$ & $\begin{array}{r}458.00 \\
+0 /-1.0 \\
\end{array}$ & $\begin{array}{l}41.0 \\
+0 /-1.0\end{array}$ & $\begin{array}{l}764.11 \\
\pm 0.05\end{array}$ & $401 \pm 0.05$ \\
\hline
\end{tabular}

\subsection{Large-aperture diagnostic system (LADS)}

\subsubsection{Overview}

We and our colleagues from the French CEA designed and built an optical diagnostic system to perform time-resolved gain and wavefront measurements over the entire aperture of our prototype amplifier. Measurements were performed using a pulsed, injection seeded, single-longitudinal mode, Nd:YLF probe laser operating at $1.053 \mu \mathrm{m}$. This laser produced 40-mJ, 20-ns-long pulses at a repetition rate of $13 \mathrm{~Hz}$. The probelaser beam was expanded and image relayed by a series of telescopes. After passing through the amplifier once, the beam was reflected by a mirror, then passed back through the amplifier and telescopes a second time. After returning from the amplifier, a beam splitter reflected a portion of the beam to scientific-grade charged coupled device (CCD) cameras for gain and wavefront measurements. Figure 3.4 shows a schematic diagram of our equipment.

Amplifier gain distributions were determined by calculating the ratio of the images produced by two CCD cameras (GR and GP in Figure 3.4) that recorded the laser beam intensity distribution before and after the beam passed through the amplifier. Measured background contributions from the flashlamp light and amplified spontaneous emission were subtracted from the gain camera image. A correction factor for passive transmission losses was determined by firing the probe laser without firing the amplifier flashlamps. Cross hair images were used to ensure proper registration of the gain and reference camera images. 


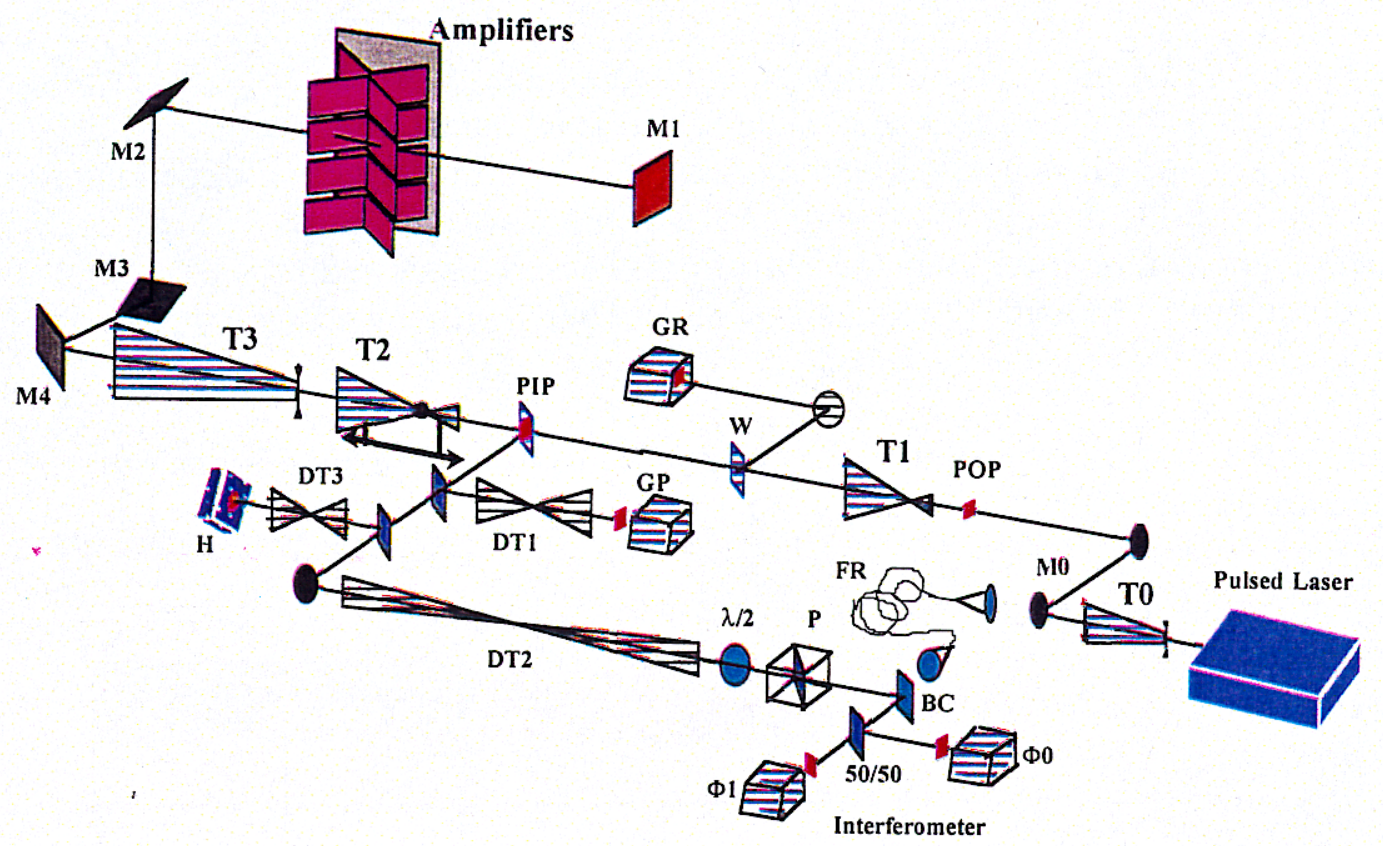

Figure 3.4. Large-aperture diagnostic system schematic.

Wavefront distributions were generated using a modified Twyman-Green interferometer. The reference beam was generated by injecting a sample of the probe beam into a 25 -m-long single-mode optical fiber (FR) cut so that its optical length approximately matched the probe-laser path length. To increase the fringe contrast ratio, a half-wave plate and a polarizer were used to attenuate the probe laser to match the intensity of the reference beam. Two cameras were used to record interferograms on successive pulses of the $13-\mathrm{Hz}$ probe laser. Wavefront distributions were calculated from the interferograms by performing a discrete Fourier transform on the raw CCD cameras, extracting the phase information from a predetermined region of the Fourier spectrum, relocating the extracted signal to the origin, and performing an inverse Fourier transform. Prompt pump-induced wavefront distortion was determined by subtracting the wavefront measured at the time of peak gain from the wavefront measured $75 \mathrm{~ms}$ earlier. The calibration of the interferometer was checked by measuring the wavefront with and without a known lens inserted in the beam path. From this check, we estimate the accuracy for relative wavefront measurements to be \pm 0.02 waves rms.

The remainder of this section provides more detailed information about the hardware and design of the LADS system. For descriptions of analysis techniques and measurement results, the reader is referred to Section 4 for gain data, to Section 5 for prompt pump-induced wavefront data, and to Section 6 for thermal recovery data.

\subsubsection{Detailed descriptions}

\section{Probe laser}

The pulsed probe laser was built by Quantel for the French CEA. This probe laser used two flashlamp-pumped Nd:YLF rods, one for an oscillator and one for an amplifier. 
The flashlamps and pump cavities were water cooled to enable continual $13-\mathrm{Hz}$ operation. TEM $\mathrm{T}_{00}$ transverse-mode operation was obtained by placing a round aperture in the oscillator cavity and by using diffuse reflectors in the flashlamp cavities to achieve a uniform pumping of the laser rods. Single-frequency operation was achieved by seeding the oscillator with the output from a diode-pumped Nd:YLF microlaser with an internal Fabry-Perot etalon. The laser oscillator was Q-switched with a Pockels cell and an intracavity polarizer. To ensure single-longitudinal-mode operation, a closed feedback loop tuned the oscillator cavity length for minimum pulse build-up time by adjusting the oscillator cavity length with a piezo-electric actuator on the back-mirror. A second Pockels cell switch and polarizer combination at the output was used to reject the offwavelength hole burning after pulse. Table 3.2 gives measured performance parameters and a brief description of the measurement method.

Table 3.2. Performance characteristics of the custom-made Quantel pulsed Nd:YLF laser.

\begin{tabular}{|c|c|c|}
\hline Performance characteristic & Value & Measurement method or tool \\
\hline Average output energy & 40 to $80 \mathrm{~mJ}$ & Calorimeter \\
\hline Pulselength & 17 to $25 \mathrm{~ns}$ & Fast photodiode/oscilloscope \\
\hline Beam diameter & $\begin{array}{l}3.6 \mathrm{~mm} \text { at } 1 / \mathrm{e}^{2} \\
\text { intensity points }\end{array}$ & Spiricon beam analyzer \\
\hline Beam divergence & Diffraction limited & Spiricon beam analyzer \\
\hline Pointing stability & $\begin{array}{l} \pm 15 \mu \mathrm{rad} \text { at the } \\
\text { output of laser }\end{array}$ & Spiricon beam analyzer \\
\hline $\begin{array}{l}\text { Shot-to-shot variation in } \\
\text { output energy }\end{array}$ & $-5 \%$ & Fast photodiode/oscilloscope \\
\hline Output fluence distribution & Gaussian & Spiricon beam analyzer \\
\hline Wavelength & $1.053 \mu \mathrm{m}$ & By Quantel \\
\hline
\end{tabular}

\section{Beam expansion and image relaying}

Referring to Figure 3.4, a series of four telescopes magnified the 3.6-mm-diam probe laser beam produced by the Quantel laser by a factor of 240 before the beam was double passed through the prototype amplifier. Telescope T0 was a Newtonian telescope with $2 \times$ magnification, which relayed an image of the probe laser beam waist to the principal object plane (POP). Cross hairs, apertures, and reticles were inserted at the POP to aid alignment. Telescope $\mathrm{T} 1$ was a Galilean telescope with $6 \mathrm{x}$ magnification, which relayed the image from the POP to beamsplitter B2 as well as to the reference gain camera, GR. T2, a Galilean telescope, and T3, a Newtonian telescope, had 5.38x and 3.5x magnification, respectively, and together relayed the image from beamsplitter $B 2$ to mirror M1. Telescope T2 was on a linear translation stage so that the image plane at beamsplitter B2 could be relayed to mirror M1 regardless of which of the eight amplifier apertures was being probed. Depending on the aperture, the optical path length from 
beamsplitter B2 to mirror M1 varied from 24 to $30 \mathrm{~m}$. The probe beam, which had a Gaussian intensity distribution, overfilled the 40-cm-square amplifier aperture and was clipped at $\sim 50 \%$ of the peak intensity at the middle of the edges and at $\sim 25 \%$ of the peak intensity at the corners.

After passing through the prototype amplifier once, the beam was reflected by mirror $\mathrm{M} 1$ and passed through the prototype amplifier a second time. Telescopes T3 and T2 demagnified the beam and relayed the image back to beamsplitter B2. In turn, the image at B2 was relayed to the gain camera, GP, by diagnostic telescope DT1; to the two interferometer cameras, $\Phi 0$ and $\Phi 1$, by diagnostic telescope DT2; and to the Hartmann sensor, $\mathrm{H}$, by diagnostic telescope DT3. Both DT1 and DT2 had unity magnification, while telescope DT3 had $2.5 \times$ demagnification.

To avoid air breakdown, telescopes $\mathrm{T} 1$ and $\mathrm{T} 2$ were vacuum telescopes. To reduce contributions to the camera-image signals by flashlamp light and amplified spontaneous emission (ASE) from the amplifier, a pinhole filter with a diameter of $\sim 100$ times the diffraction-limited spot size was inserted in T2. This filter limited the spatial resolution of the gain and wavefront measurements to $\sim 4 \mathrm{~mm}$ in the plane of the amplifier.

Beam propagation calculations were performed with Super-Oslo to design the aspheric lenses used in the telescopes. The calculations showed that the lenses would add less than three waves of static distortion to the beam.

\section{Large turning mirrors and their mounts}

The four largest turning mirrors, M1, M2, M3, and M4, deserve special attention due to their potential for affecting probe-beam wavefront and pointing stability. Mirrors M2 and $\mathrm{M} 3$ were oriented at $45^{\circ}$ with respect to horizontal, and both mirrors were affected by gravity sag. Since mirror M2 faced upwards while M3 faced downwards, each mirror's sag was partially compensated for by the sag of the other. This compensation was imperfect, however, as the tilt angles of M2 and M3 were orthogonal in the horizontal plane (see Figure 3.4). Calculations showed the combined gravity sag of the two doublepassed mirrors would produce $\sim 3$ waves of mostly astigmatic distortion, which accounts for most of the measured static wavefront distortion in the LADS system. To reduce gravity sag, $10-\mathrm{cm}$-thick substrates were used for the 45-degree mirrors, while an $8-\mathrm{cm}$ thick substrate was used for the vertically oriented, normal-incidence mirror M1. All four mirror surfaces had a finishing error requirement of less than one-sixth wave distortion peak-to-valley, while the reflectance at the operating angles was required to be $>99 \%$ at $1.053 \mu \mathrm{m}$.

Figure 3.5 shows the mirror mount for M3. Similar rotating mounts were used to support the other three large mirrors. The tilt angle was adjusted using a stepping motor. The mirror mounts were bolted to linear translation stages, which in turn were bolted to the mirror tower enclosures. The translation stages for M2 and M3 had large enough range so that any one of the eight prototype amplifier apertures could be accessed by the probe beam. 


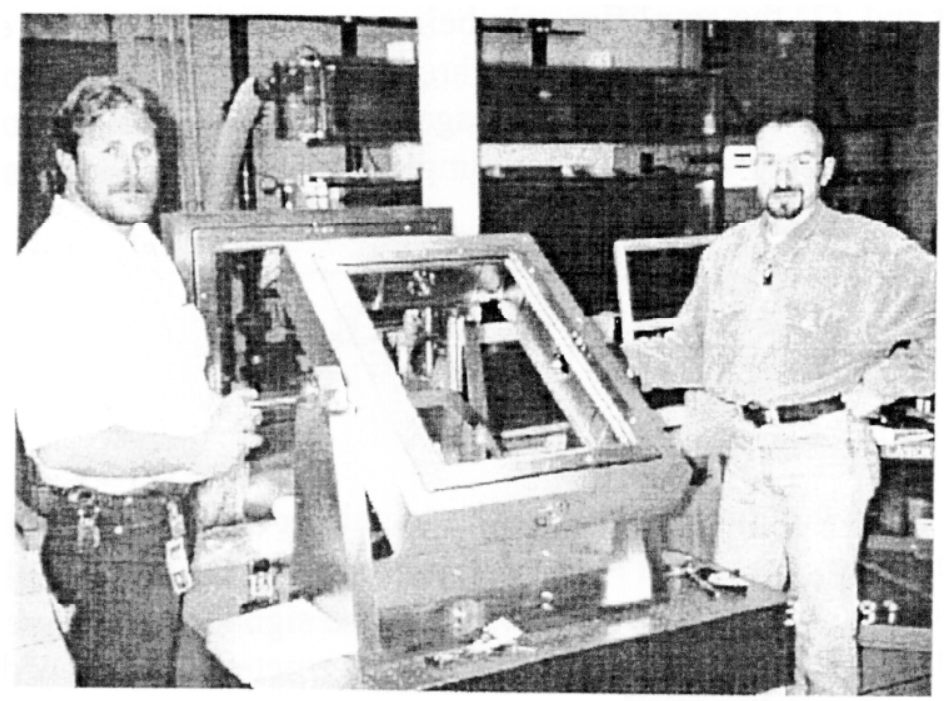

Figure 3.5. Mirror mount used to support mirror M3.

The mechanical stability of the mirrors and their mounts was especially important, since demagnification of the beam by the telescopes caused beam pointing variations generated by the large mirror vibrations to be 20 times larger at the gain and wavefront cameras. The mirror-tower enclosure, translation stages, and mirror mounts were designed using finite-element analysis, which showed that each mirror would meet its requirement of adding $<2 \mu \mathrm{rad}$ of beam steering due to random vibrations. This analysis used driving terms estimated from vibration spectra measured in AMPLAB.

\section{Gain cameras and images}

A major objective of our amplifier development effort is to develop accurate models for predicting the average gain coefficient and gain distributions produced by the NIF amplifiers. Due to the similarity of the AMPLAB prototype amplifier to the NIF amplifiers (see Sections 2 and 4), measured gain distributions could themselves serve as predictors for NIF amplifier performance. Accordingly, we set out to measure the gain coefficients of the AMPLAB prototype amplifier with an accuracy and precision of $\pm 1 \%$. This level of uncertainty in the data was judged appropriate for both deriving NIF predictions from AMPLAB data and the benchmarking of our codes.

At the outset, the error associated with the gain coefficient distribution measurement could be separated into two basic components: instrumental noise and beam quality. The instrumental noise component was tractable and was in fact analyzed. The blemishes in the optics including damage sites on the slabs diffract the probe beam inducing what we refer to as the beam-quality-induced error. The beam quality error varied from aperture to aperture and was exacerbated by the pointing instability of the probe laser. To diminish the beam quality error a smoothing technique was employed (see Section 4) on the data. The beam quality error, dominated the intrinsic instrumental error.

The intrinsic instrumental error stems from three basic sources: (1) the stochastic (electron well) shot noise in the camera pixels, (2) the optical background due tơ ASE 
and flashlamp light and, (3) the amplifier gain being measured. The error engendered by these physical measurement limits propagates through the mathematical operations for computing the gain coefficient from several images. At the end of our instrumental error analysis (not including beam quality error), a simple formula for the intrinsic relative error $\varepsilon$ in the gain coefficient was derived,

$$
\varepsilon \approx \frac{\delta}{\ln (G)} \cdot \sqrt{\frac{x+2}{x+1}\left(1+\frac{P_{A S E}}{I_{0}}+\frac{P_{F L}}{G \cdot I_{0}}\right)^{2}+1}
$$

where, $\delta$ is the shot noise component, $\mathrm{G}$ is the large gain, $\mathrm{x}$ is the measured (optical) signal to background ratio; $P_{A S E}$ and $P_{F L}$ are signal contributions from amplifiedspontaneous emission and flashlamp optical background signals, respectively; and $\mathrm{I}_{\mathrm{O}}$ is the probe laser input signal. The interaction of these parameters originally dictated our selection of LADS components critical to minimizing the measurement error. We selected the pinhole in T2 and the laser energy to minimize the quantity inside the square root in formula (3.1) Of course, the pinhole in T2 limited the amount of optical background (estimated from first principles) reaching the gain CCD (GP in Figure 3.4) but it also limited the spatial resolution. A spatial resolution of $4 \mathrm{~mm}$ was considered sufficient for our purposes; thus a pinhole 100 times the diffraction limit was selected for telescope T2. The optical background amounted to about $0.1 \%$ to $0.4 \%$ of the signal levels depending on the gain being measured and the position in the aperture. In the limit of large $\mathrm{I}_{O}$ (i.e., a strong probe laser relative to the optical background), $\varepsilon$ is given by the ratio of the shot noise $\delta$ to the natural $\log$ of the amplifier gain measured. Using the expected value for the amplifier gain, we chose the CCD electron wells to be as deep as the state of the art allowed (350,000 electrons per pixel). The relative shot noise error $(\delta)$ was about 1 part in 500 for pixels near the center of the image (where the electron wells nearly saturated) and it varied to 1 part in 250 at the corner of the images where the intensity was about $1 / 4$ that at the center. Because the extent of each pixel corresponded to about $0.4 \mathrm{~mm}$ of the amplifier aperture, further reductions in this intrinsic error were obtained by numerically collecting $10 \times 10$-pixel regions without impacting the spatial resolution. The shot noise error calculated when the $10 \times 10$ "bins" were used resulted then in a $\delta$ of 1 part in 1500 at the center and 1 part in 750 at the corners of the aperture.

\section{Gain calibration}

As a check of the gain measurement, calibrated neutral density filters (NDFs) were inserted in the probe beam path. The NDFs were selected to approximately match the inverse of the gain levels being measured so that the signal levels in the CCDs were similar to those observed during the gain measurements. The calibration of the NDFs was done using pyroelectric detectors used for bulk gain measurements (not shown in Figure 3.4 but in positions equivalent to the gain cameras GR and GP). The manuals for these pyroelectric detectors specified an error of $0.1 \%$ for a single-pulse measurement. The average of 100 laser pulses was used to calibrate the NDF filters. The measurement of the ND filters using the CCD gain cameras agreed with the calibration measurements to within $0.25 \%$. 


\section{Interferometer}

Interferometer measurements were carried out simultaneous to gain measurements. Interference fringes were obtained by combining the probe beam's near field image with a nearly perfect reference wavefront derived from a polarization maintaining single mode fiber. Two $1024 \times 1024$ scientific cameras situated at conjugate relay planes collected the interference fringes. The two cameras could be triggered independently. We were able to observe (after data processing) the wavefront change in consecutive laser pulses spaced at integral multiples of $75 \mathrm{~ms}$ (the pulse-to-pulse spacing). Two components contributed to wavefront errors in our NIF-prototype amplifiers: (1) a slowly changing "material" component associated with temperature gradients in the slabs; and (2) a rapidly changing, stochastic component associated with temperature gradients and movement of the gas within the beam tubes and amplifiers assemblies.

For prompt wavefront distortion measurements, the wavefront measured $75 \mathrm{~ms}$ before the time of peak gain was subtracted from the wavefront measured at the time of peak gain. The 75-ms delay was found to be sufficiently small to "freeze" gas motion, and an error of \pm 0.02 waves rms was typical. Phase error increased at longer delays, consistent with gas motion effects. In thermal recovery experiments before and after a shot and at regular (one-hour to three-hour) intervals, interferogram pairs were obtained with delays between the two cameras of 0 seconds, $75 \mathrm{~ms}, 1$ second, and 10 seconds. In addition, sets of 20 interferograms spaced 10 seconds apart were collected. These sets of 20 were averaged for better statistics, and the average was compared to its components to obtain experimental P-V and rms measures. These numbers were found to correlate with temperature and/or gas motion effects during thermal recovery. The rms and P-V were also sensitive to external causes out of our control, for example diurnal cycles during hot days and AMPLAB VIP tours that on occasion increased measurably the hot air in and around the amplifier after the use of show and tell flood-lights!

Referring to Figure 3.4, the probe image at PIP was relayed to the modified TwymanGreen interferometer by the one-to-one diagnostic telescope DT2. At the output of DT2, a half-wave plate followed by a cube polarizer acted as an intensity control to balance the probe laser signal with the much weaker reference signal that emerged from the output end of fiber reference (FR). The probe image was combined with the reference wavefront at the beam combiner (BC). The reference wavefront was produced as follows: a coherent sample of the input beam was collected behind the turning mirror $\mathrm{M} 0$. To avoid damaging the input face of the fiber FR, the energy of this sample was further diminished with neutral density filters (not shown) to a level of $\sim 20 \mu \mathrm{J}$. A half wave plate (not shown) was used to align the polarization to the input of fiber FR. A 20x microscope objective focused the beam and overfilled the $6-\mu \mathrm{m}$ core of FR. An xyz stage was used to locate the input end of FR at focus. The length of fiber FR was chosen to provide the necessary delay corresponding to the probe beam time of flight to and from the back mirror M1. The overlap in time of the arrival of reference and probe signals at $\mathrm{BC}$ was verified (and the fiber length adjusted as necessary) with a fast photodiode. At the output end of fiber FR, a 20-cm focal length lens was used to collect the single mode beam that emerged. A shear plate was initially used to collimate the reference beam. A half wave plate following the collimating lens was used to align the polarization to 
coincide with the probe beam polarization. A Glan-Thompson polarizer was momentarily inserted for this purpose. The output energy of the fiber was found to be adequate for the scientific cameras. The nonlinear interaction of the 20 -ns, singlefrequency laser pulse within the 24-m-long fiber core limited the output of the fiber. The input vs output characteristics were measured for a few input energies. The output of the fiber did not scale linearly with the input (see Table 3.3). Based on the nonlinear coefficients for fused silica, we estimated that stimulated Bruillion scattering and to a lesser extent Raman scattering limited the output. At an output of $\sim 6 \mu \mathrm{J}$ the reference fiber provided reliable, long-term operation.

Table 3.3. Input vs output characteristics for the 24-m-long reference fiber.

\begin{tabular}{|c|c|}
\hline Input $(\mu \mathrm{J})$ & Output $(\mu \mathrm{J})$ \\
\hline 5 & 2.8 \\
\hline 10 & 4.5 \\
\hline 20 & 6.3 \\
\hline 30 & 7.3 (damage after $\sim$ days) \\
\hline$>30$ & Immediate damage \\
\hline
\end{tabular}

\section{Interferometer calibration}

Calibration of the interferometer was verified by measuring the wavefront of a lens with a known wavefront. This lens was inserted in the beam returning from the amplifier at a position near beamsplitter B2. The measured wavefront added by the lens agreed to within $\sim 2$ to $5 \%$ with independent measurements performed using a Zygo interferometer (see Figure 3.6). 

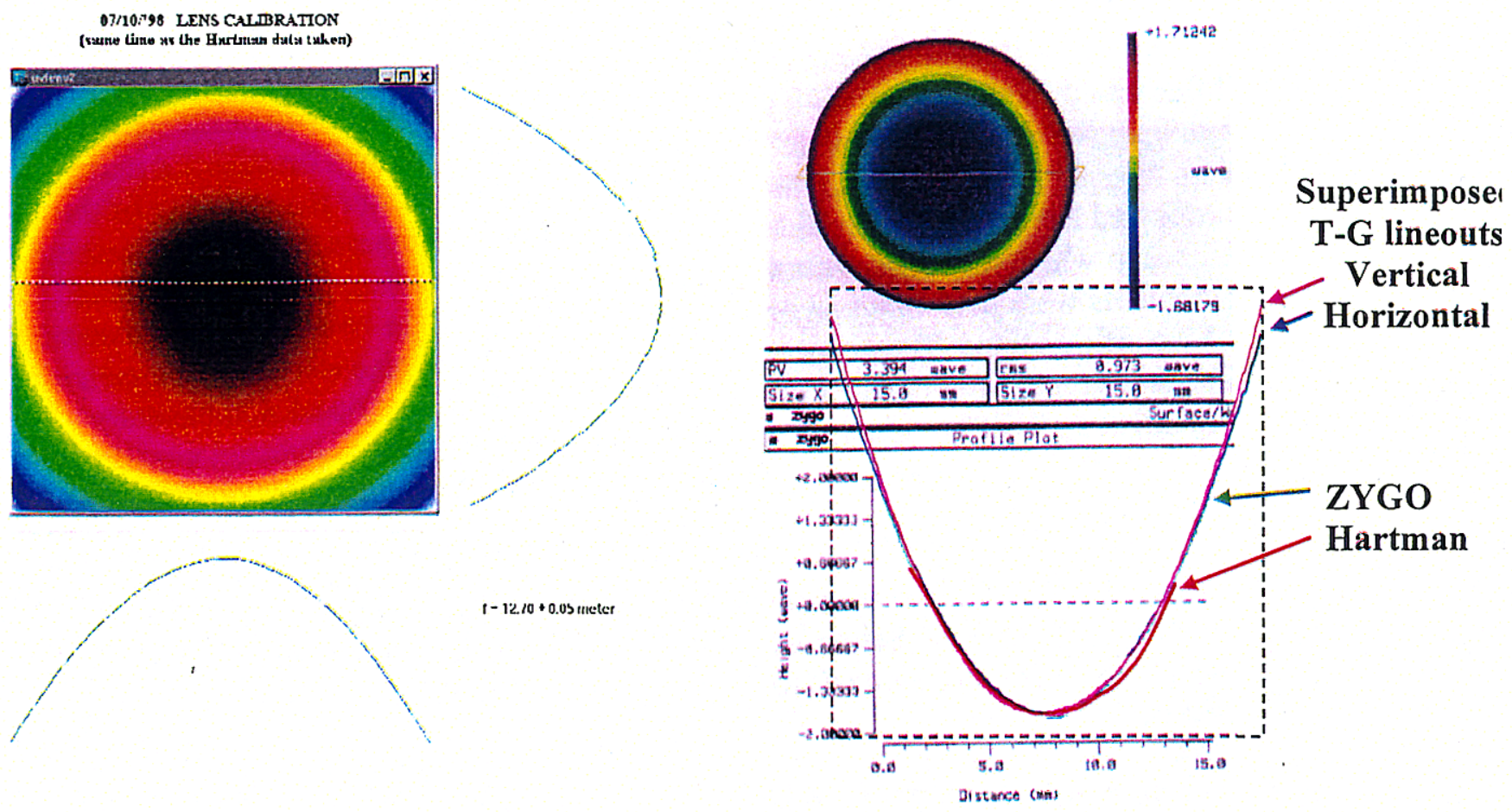

Figure 3.6. Measurements of a lens by a commercial Zygo interferometer, a Hartmann-Shack wavefront sensor and our modified Twyman-Green interferometer, were consistent within $\sim 5 \%$.

\subsection{Test configurations}

Various amplifier configurations were tested by inserting flashlamp cassettes and slab cassettes into selected locations in the framework. For example, inserting slab cassettes and flashlamp cassettes into the middle frame assembly unit, while leaving the two end frame assembly units unoccupied, produced a two-slab-wide, one-slab-long "V" amplifier configuration. Inserting slab cassettes and flashlamp cassettes into two adjacent frame assembly units, while leaving the remaining end frame assembly unit unoccupied, produced the two-slab-long "diamond" and "X" amplifier configurations. The "V", "diamond", and " $X$ " configurations are named for the shapes formed by the laser slabs (see Figure 3.7).
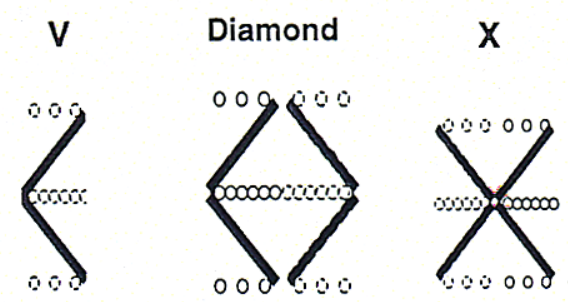

$$
3 \text { - slab - long }
$$

Figure 3.7. We have tested several prototype amplifier in the diamond, $X$, and 3-slab-long configurations.

Testing a $4 \times 2$, three-slab-long amplifier was problematic, however. For budgetary reasons, the $A M P L A B$ power-conditioning system was only large enough to drive a maximum of 48 flashlamps, 12 less than the full complement of 60 flashlamps. To 
reduce costs further, only four side flashlamp cassettes, two fewer than the six needed to outfit a full $4 \times 2$, three-slab-long amplifier, were purchased. The three-slab-long amplifier was therefore tested with all three side flashlamp cassettes missing on one side (optical inactive side). We installed absorbing architectural glass (Greylight) dummy slabs on the side of the amplifier missing the flashlamps to reduce the intensity of the light reflected back to the active side through the central flashlamp array. To determine the degree to which the missing flashlamps affected performance, gain and wavefront measurements were performed on one-slab-wide analogs of the two-slab-long "diamond" and " $X$ " configurations. These were formed by removing the slab cassettes at the ends of the 3-slab-long amplifier one at a time. As discussed in sections 4 and 5, the results showed only small differences in measured gain and prompt pump-induced wavefront distortion relative to measurements made previously on fully energized "diamond" and " "X" amplifiers.

Optical performance was measured only on the side of the amplifier with the full complement of flashlamps. Schott LG-770 laser slabs were installed in the three lower apertures, while a combination of Hoya LHG-8 and LG-770 slabs were installed in the top aperture.

\subsection{Power conditioning system}

\subsubsection{Design}

Although the AMPLAB power-conditioning system used a different architecture than the NIF power-conditioning system, it was designed to produce flashlamp input pulse energies and pulselengths close to those anticipated for the NIF. The power-conditioning system consisted of six modules, with each module using a common main switch and a common preionization circuit to energize up to four series pairs of flashlamps (see Figure 3.8). The main switches used two Thompson 8900 -series ignitrons connected in series to reduce the prefire rate, while the preionization switches used a single Thompson " $A$ " ignitron. The main circuit capacitance and inductance for each lamp pair were $310 \mu \mathrm{F}$ and $28 \mu \mathrm{H}$ inductors, respectively, with additional inductance and resistance contributed by the 40 to 50 -m-long custom made cables that connected the inductors to the flashlamps. These cables had measured capacitance, inductance, and resistance of 112 $\mathrm{pF} / \mathrm{m}, 177 \mathrm{nH} / \mathrm{m}$, and $1.0 \mathrm{mohm} / \mathrm{m}$, respectively. Each preionization circuit used a single $50 \mu \mathrm{F}$ capacitor to drive all four lamp circuits. System components were chosen to operate reliably at capacitor charging voltages up to $27 \mathrm{kV}$. 


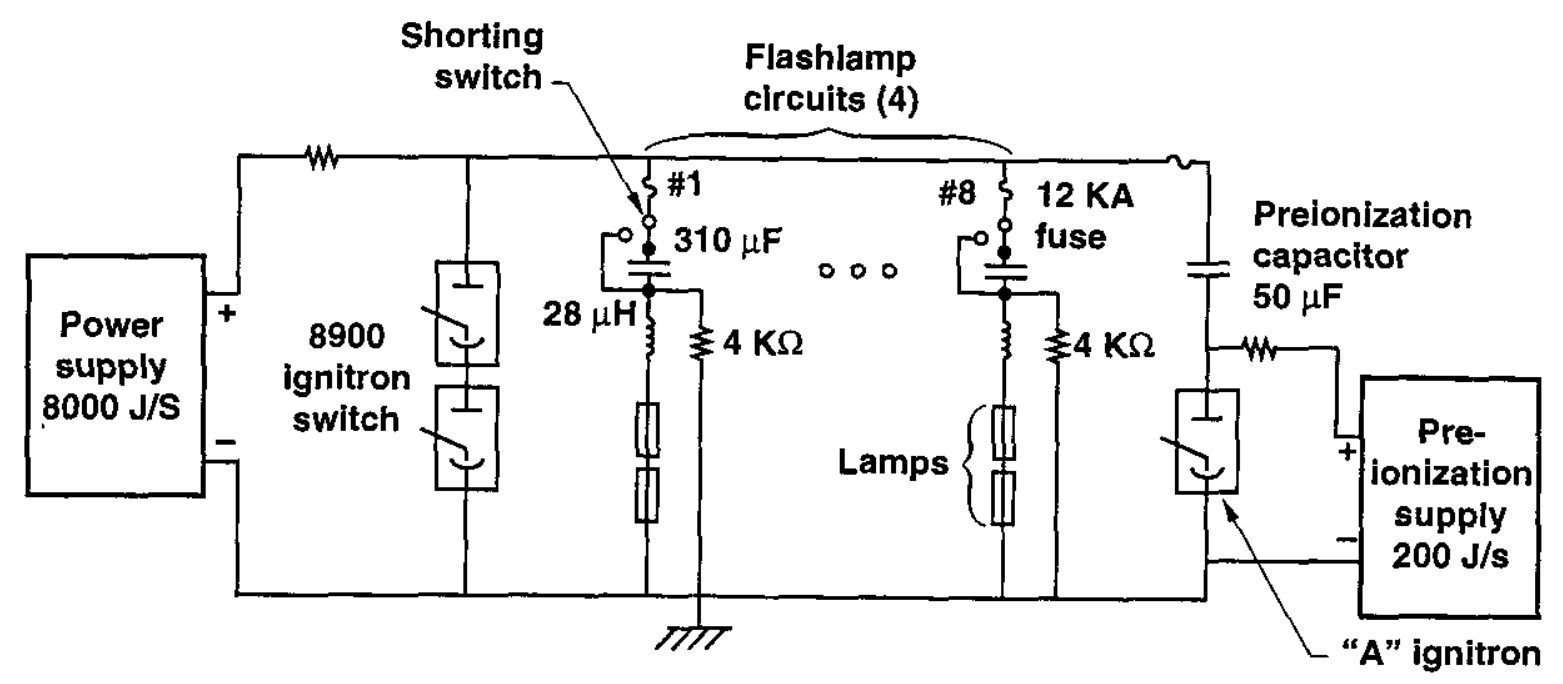

Figure 3.8. Schematic diagram of the AMPLAB power-conditioning circuit.

The voltage and current measurements were performed with the amplifier in the three-slab-long configuration, with side flashlamp cassettes activated on one side of the amplifier only. Figure 3.9 shows a plan view of the amplifier with labels showing the correspondence between flashlamp circuits and flashlamp positions. Power-conditioning modules were assigned numbers 1 through 6 , while the individual flashlamp pairs within a module were assigned letters A through D. A one-to-one correspondence between power-conditioning modules and flashlamp cassettes was maintained. Accordingly, the modules connected to the six-lamp side flashlamp cassettes had only three active circuits (A through C), while modules connected to the four-lamp central flashlamp cassettes had four active circuits. Measurements were performed on only twenty of the twenty-one flashlamp pairs, however, because the flashlamps in one pair (circuit 1B) failed to fire during the voltage and current measurements. This circuit functioned normally during the optical performance tests, however.

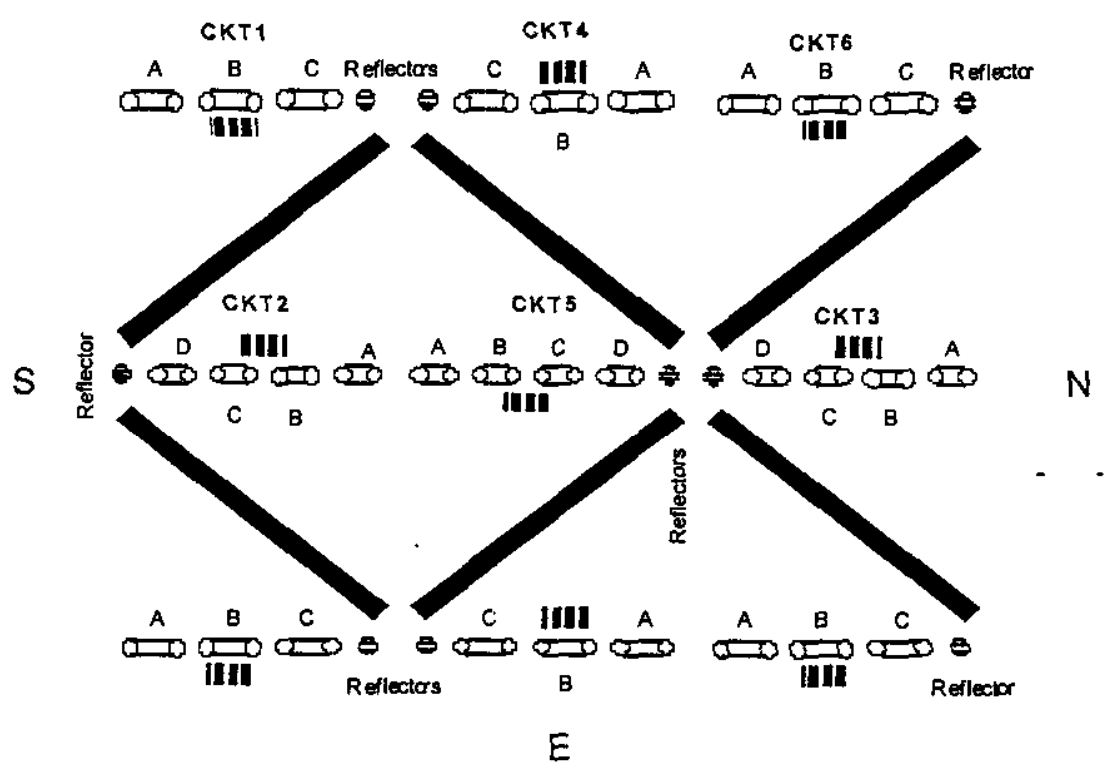

Figure 3.9. A plan view of the amplifier with labels showing the correspondence between flashlamp circuits and flashlamp positions. 


\subsubsection{Flashlamp Electrical Measurements}

\section{Experimental setup}

Voltage and current measurements were performed on 20 flashlamp pairs used in the one-slab-long, three-slab-long NIF prototype amplifier tested in AMPLAB. The measurements were made using Tektronix P6015A high-voltage probes connected across each flashlamp pair, and with Pearson $301 \mathrm{X}$ current transformers mounted close to the main circuit inductor in the capacitor bank room (see Figure 3.10). Tektronix 5:1 and $10: 1$ attenuators were used to reduce the current-transformer signals to levels readable by the oscilloscopes. Tektronix TDS644 oscilloscopes were used to digitize both voltage and current signals at a rate of $5 \mathrm{MHz}$ with 8-bit resolution.

Signals were processed using the QuickDig software package developed by Steve Fulkerson. The voltage drop across each flashlamp pair was calculated by subtracting the voltage measured on the current return side from the voltage measured on the highvoltage input side. Instantaneous electrical input power to the flashlamp was calculated by multiplying the voltage drop by the current. Electrical energies delivered during preionization and main pulses were calculated by integrating the power curves.

Voltage measurements were performed one flashlamp cassette at a time, with sufficient probes used to monitor all three or four flashlamp pairs simultaneously. Current measurements were performed using probes that remained resident in each lamp circuit. Measurements were performed over a minimum of nine shots, three at each of the three different capacitor charging voltages used for amplifier performance tests. After each nine-shot series, the voltage probes were moved to the next flashlamp cassette, and the series was repeated.

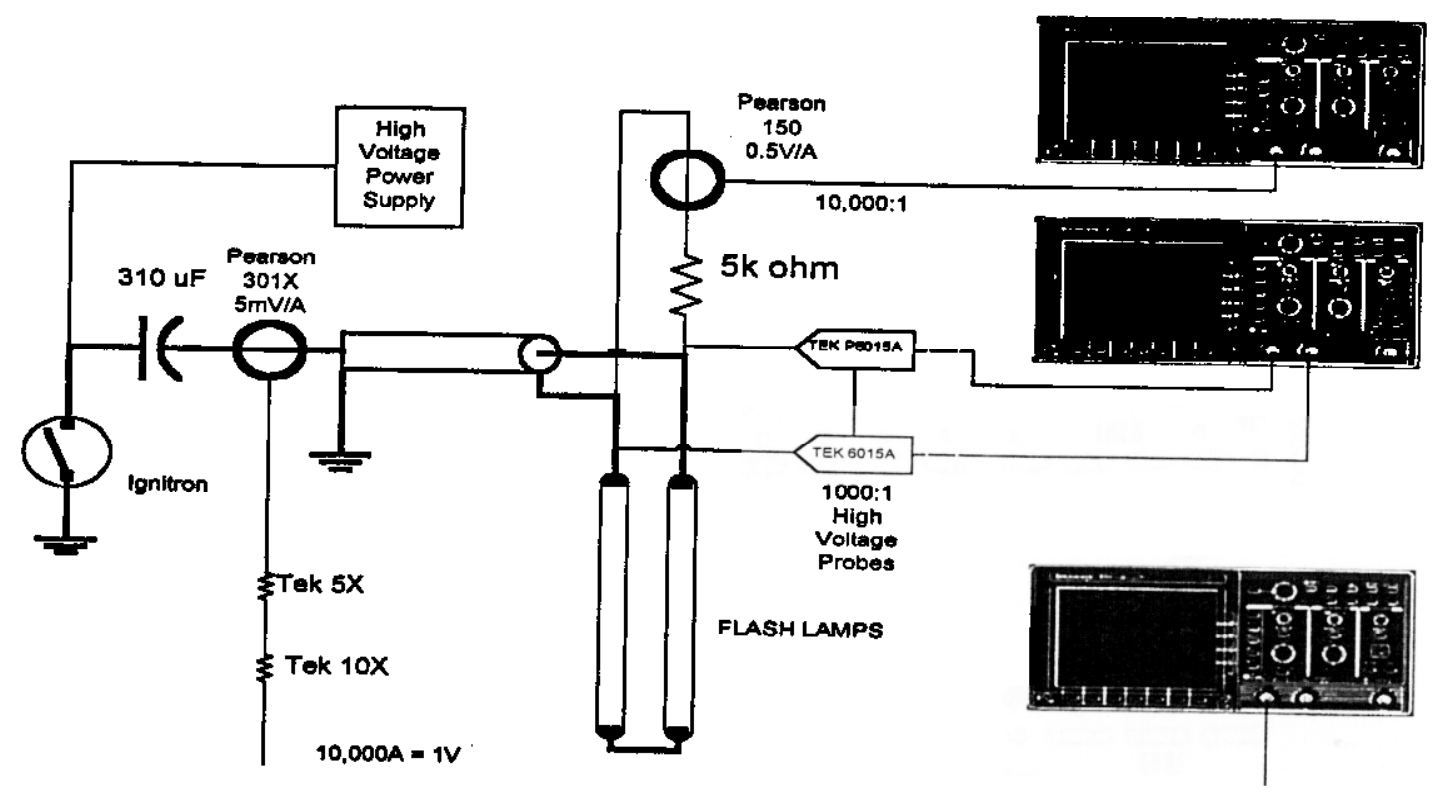

Figure 3.10. Experimental setup for measurements made on 20 flashlamp pairs. 


\subsubsection{Calibrations and error estimates}

Relative uncertainties for voltage- and current-measuring channels were estimated from the root-sum-square (rss) of the relative uncertainties of the components (i.e., probes, attenuators, and oscilloscopes), which were provided by manufacturers. See Table 3.4. Resulting uncertainties for voltage, current, and power measurements were $\pm 4.7 \%, \pm 2.3 \%$, and $+15.3 \%$, respectively.

Table 3.4. Model numbers, calibration constants, and manufacturers' estimated relative measurement uncertainty for the high-voltage probes, current probes, attenuators, and oscilloscopes. Corresponding uncertainties for voltage, current, and power measurements are $\pm 4.7 \%, \pm 2.3 \%$, and $+/ 5.3 \%$, respectively.

\begin{tabular}{|l|l|l|r|}
\hline Component & Model \# & $\begin{array}{l}\text { Calibration or } \\
\text { attenuation } \\
\text { constant }\end{array}$ & $\begin{array}{l}\text { Manufacturer's } \\
\text { estimated } \\
\text { uncertainty }\end{array}$ \\
\hline High-voltage probe & Tektronix P6015A & $1000: 1$ & 0.03 \\
\hline Current probe & Pearson 301X & $200 \mathrm{~A} N$ & 0.01 \\
\hline $\begin{array}{l}\text { Oscilloscope: } \\
\text { signal amplitude }\end{array}$ & Tektronix TDS644 & variable & 0.015 \\
\hline $\begin{array}{l}\text { Oscilloscope: } \\
\text { timebase }\end{array}$ & Tektronix TDS644 & variable & 0.0001 \\
\hline Attenuator & $\begin{array}{l}\text { Tektronix 011- } \\
0059-02\end{array}$ & $10: 1$ & 0.01 \\
\hline Attenuator & $\begin{array}{l}\text { Tektronix 011- } \\
0060-02\end{array}$ & $5: 1$ & 0.01 \\
\hline
\end{tabular}

We tested the voltage probes by connecting all eight to the same lamp pair and determining the average voltage produced by each probe over three shots. The standard deviation of the signals produced by the eight probes was $1.2 \%$, about one-third the experimental uncertainty estimated from the rss of the component uncertainties. The current probes were tested in a slightly different manner, as it was impractical to install more than two current probes on the same flashlamp-pair circuit. Rather, a single current probe, which was randomly selected from the group of available probes, was moved from circuit to circuit, for the three-shot tests. The signals produced by the moving reference probe were compared with the signals produced by the resident probes, using ratios of the probe signals averaged over a $200 \mu$ s-long period approximately centered at the time of peak current. The average ratio of the reference probe signal to resident probe signals was 1.0058 with a standard deviation of $0.75 \%$, also about one-third the rss estimate. The narrow clustering of both the voltage and current probes suggests that uncertainties provided by the manufacturers are conservative with respect to probe-to-probe variations. However, our test does not rule out a possible systematic bias in the probe calibrations.

The calibration constants were adjusted to improve experimental accuracy. Specifically, the calibration constants of the individual voltage-measurement channels (i.e., probe and oscilloscope) were adjusted to agree with the average of the other eight 
channels. Similarly, the calibration constants for the twenty current-measurement channels were adjusted to agree with the group average.

\subsubsection{Results}

The electrical energies delivered during the main pulses were converted into the explosion fraction, $f_{\mathrm{x}}$, which was calculated from measured delivered energy $E_{\text {del }}$ using the formulas

$$
f x=\frac{E d e l}{E x}
$$

and

$$
E_{\mathrm{r}}=20,000 L D \sqrt{\frac{\tau}{3}}
$$

where $E_{x}$ is the single-shot explosion energy for a flashlamp operating in the open in Joules, $\mathrm{L}$ is the flashlamp arc length in centimeters, $\mathrm{D}$ is the flashlamp bore diameter in centimeters, and $\tau$ is the $10 \%$ of peak power pulse width in seconds. The above expression for single-shot explosion energy normally applies when $\tau$ is the calculated $3(\mathrm{LC})^{0.5}$ pulselength. Measurements performed on flashlamps circuits near critical damping have shown, however, that the full-width tenth-max (FWTM) pulselength for the input power pulse is within a few percent of the calculated $3(\mathrm{LC})^{0.5}$ pulselength. Accordingly, explosion fractions were estimated using measured FWTM pulselengths.

Table 3.5 summarizes the measurement results for the main electrical pulses. At charging voltages of $21.05,24.30$, and $26.00 \mathrm{kV}$, the average main-pulse electrical energies were $26.3,34.7$, and $39.4 \mathrm{~kJ}$, respectively, corresponding to flashlamp explosion fractions of $0.149,0.200$, and 0.229 . The bank-to-lamp transfer efficiency was approximately $71 \%$, which is low compared with the $85 \%$ transfer efficiency previously achieved on the Nova laser and anticipated for the NIF. As is typical for such flashlamp circuits, the FWTM pulselength decreased slightly as the charge voltage was increased. Measured values at the 20.3,23.5, and $25.2 \mathrm{kV}$ charge voltages were 392,377 , and 370 $\mu \mathrm{s}$, respectively. 
Table 3.5. Summary of main-pulse measurements performed on 20 flashlamp circuits. Each entry represents the average value measured over three shots.

\begin{tabular}{|c|c|c|c|c|c|c|c|c|c|}
\hline $\begin{array}{l}\text { Charqe } \\
\text { voltaqe: }\end{array}$ & & $.05 \mathrm{kV}$ & & & $4.30 \mathrm{kV}$ & & & $6.00 \mathrm{kV}$ & \\
\hline Circuit & $\begin{array}{l}\text { EWmin } \\
\text { Puiselenath } \\
\text { (us) }\end{array}$ & $\begin{array}{l}\text { Enerav! } \\
\text { lamp. } \\
\text { (kid) }\end{array}$ & $\begin{array}{l}\text { Explosion } \\
\text { fraction. }\end{array}$ & $\begin{array}{c}\text { FWTM } \\
\text { Pulselenath } \\
(\mu s)\end{array}$ & $\begin{array}{c}\text { Eneravl } \\
\text { lamp } \\
(\mathrm{kJ}) \\
\end{array}$ & $\begin{array}{c}\text { Explosion } \\
\text { fraction }\end{array}$ & $\begin{array}{c}\text { FWTM } \\
\text { Pulselenath } \\
(\mu \mathrm{s})\end{array}$ & $\begin{array}{c}\text { Eneravl } \\
\text { lamp } \\
(\mathrm{kJ})\end{array}$ & $\begin{array}{c}\text { Explosion } \\
\text { fraction }\end{array}$ \\
\hline $1 A$ & 387 & 26.5 & 0.151 & 376 & 35.1 & 0.203 & - & 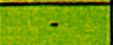 & - \\
\hline $1 C$ & 393 & 267 & 0150 & 382 & 35.2 & 0.201 & - & - & - \\
\hline ID & 392 & 265 & 0150 & 384 & 34.9 & 0.199 & $=$ & - & 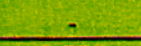 \\
\hline $2 A$ & 388 & 273 & 0.155 & 377 & 36.0 & 0.207 & 372 & 41.0 & 0.238 \\
\hline $2 B$ & 392 & 27.4 & 0.155 & 382 & 36.1 & 0.207 & 377 & 41.2 & 0.237 \\
\hline $2 C$ & 393 & 273 & 0.154 & 383 & 35.9 & 0.205 & 378 & 40.9 & 0.235 \\
\hline 20 & 394 & 271 & 0,153 & 384 & 35.4 & 0.202 & 378 & 40.6 & 0.234 \\
\hline $3 A$ & 399 & 254 & 0,142 & 375 & 33.5 & 0.192 & 372 & 37.9 & 0.220 \\
\hline 38 & 394 & 249 & 0140 & 374 & 33.0 & 0.191 & 367 & 37.2 & 0.217 \\
\hline $3 C$ & 402 & 250 & 0139 & 379 & 32.8 & 0.187 & 374 & 37.3 & 0.216 \\
\hline $4 A$ & 384 & 271 & 0.755 & 365 & 35.5 & 0.208 & 360 & 40.4 & 0.238 \\
\hline $4 B$ & 391. & & 0150 & 371 & 34.6 & 0.201 & 363 & 39.5 & 0.232 \\
\hline $4 C$ & 396 & 267 & 0.150 & 376 & 34.9 & 0.201 & 368 & 39.6 & 0.231 \\
\hline$\frac{5}{5 A}$ & 395 & 27.1 & 0.553 & 381 & 35.8 & 0.206 & 375 & 40.7 & 0.235 \\
\hline $5 B$ & 389 & 276 & 0156 & 378 & 36.3 & 0.209 & 373 & 41.3 & 0.239 \\
\hline $5 C$ & 390 & 265 & 0,150 & 376 & 35.0 & 0.202 & 373 & 39.9 & 0.231 \\
\hline 50 & 397 & 274 & 0.152 & 384 & 35.4 & 0.204 & 380 & 40.6 & 0.233 \\
\hline$\frac{}{6 A}$ & 375 & 246 & 0.142 & 359 & 31.9 & 0.188 & 353 & 36.3 & 0.216 \\
\hline $6 B$ & 393 & 247 & 0.139 & 378 & 32.6 & 0.187 & 370 & 37.3 & 0.217 \\
\hline 60 & 390. & 255 & 0.144 & 372 & 33.2 & 0.193 & 364 & 38.1 & 0.223 \\
\hline Average & 392 & 26.4 & 0.149 & 377 & 34.7 & 0.200 & 370 & 39.4 & 0.229 \\
\hline $\begin{array}{l}\text { Standard } \\
\text { deviation }\end{array}$ & 5.7 & 0.98 & 0.0056 & 6.5 & 1.34 & 0.0073 & 7.2 & 1.68 & 0.0087 \\
\hline $\begin{array}{c}\text { Nomalized } \\
\text { Standard } \\
\text { Deviation } \\
(\%)\end{array}$ & $1.5 \%$ & $3.7 \%$ & $3.8 \%$ & $1.7 \%$ & $3.9 \%$ & $3.7 \%$ & $1.9 \%$ & $4.3 \%$ & $3.8 \%$ \\
\hline
\end{tabular}

The pulse characteristics achieved at the middle charge voltage, $24.30 \mathrm{kV}$, were close to the NIF power-conditioning specifications. Specifically, the average FWTM pulselength was $377 \mu \mathrm{s}, \sim 5 \%$ greater than the NIF's specified $360 \mu \mathrm{s}$, while the average main-pulse energy was $34.7 \mathrm{~kJ}, \sim 2 \%$ greater than the NIF's specified $34 \mathrm{~kJ}$. The average flashlamp explosion fraction was 0.200 , the same as the NIF's specification. Recall that the experimental error for power and energy measurements was $\pm 5.3 \%$. Figure 3.11 shows the current, voltage, power, and impedance parameter measured on a single shot for a typical flashlamp circuit (circuit 2D on shot A80531020).

The standard deviation for lamp-to-lamp energy variations for the twenty flashlamp pairs was about $4 \%$. Most of this lamp-to-lamp variation was caused by differences between flashlamp cassettes, due to module-to-module variations in the output voltages of the high-voltage power supplies. Differences in lamp energy within the same cassette averaged $\sim 1.2 \%$, and were due to varying circuit capacitance, cable lengths, and flashlamp impedance. The average standard deviation due to shot-to-shot variations for the twenty lamp pairs was $\sim 0.7 \%$. 
Current

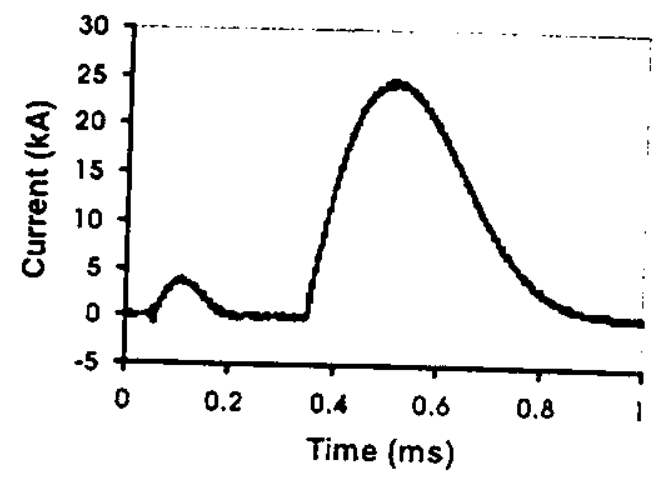

(a)

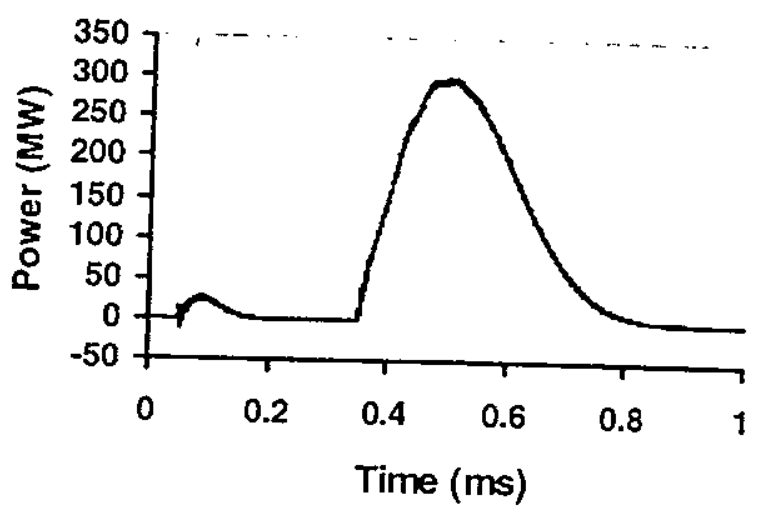

(c)

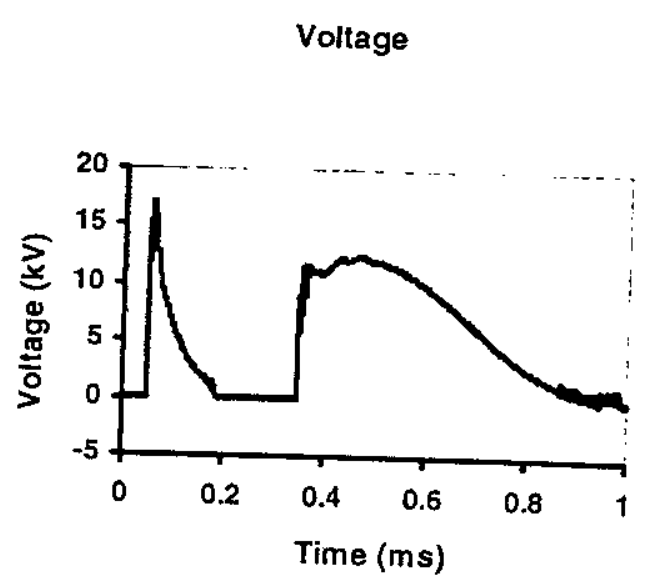

(b)

Impedance parameter

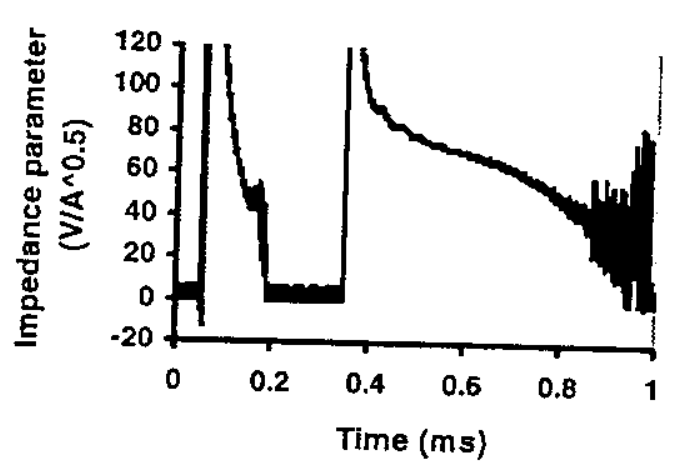

(d)

Figure 3.11. Measured (a) current, (b) voltage, (c) power, and (d) impedance parameter for an $f_{x}=$
0.2 shot. 


\section{Gain Data and Modeling}

\subsection{Gain Data Reduction}

The gain profiles measured on the NIF prototype amplifier in AMPLAB were calculated from the $C C D$ camera images provided by the large-aperture diagnostic system, which is described in Section 3.2. A total of seven images were required for each gain profile calculation. First, a cross hair was placed in the input beam, and its image on both the input and output cameras (GR and GP in Figure 3.5) was used to register the pixels on the two cameras to each other. Next, pre-shot images of the probe beam on the input camera (identified as signal $A_{p}$ ) and the output camera (signal $B_{p}$ ) were taken without firing the flashlamps. These images were used to normalize the camera outputs at zero gain. To remove the signal seen by the output camera coming from ASE and flashlamp light, a background image (signal $B_{b}$ ) was acquired at the time of peak gain where the flashlamps were fired, but the probe beam was blocked. Finally, a shot was taken with the probe beam passing through the amplifier at peak gain, and its images on both the input $\left(A_{s}\right)$ and output $\left(B_{s}\right)$ cameras were extracted.

Once all the images were correctly registered using the cross hair data, the gain profile $(\mathrm{G})$ was calculated via the expression

$$
G=\frac{\left(B_{s}-B_{b}\right)}{A_{s}} \frac{A_{p}}{B_{p}}
$$

The profile of the average gain coefficient $\alpha$ can then be expressed in terms of the logarithm of the gain:

$$
\alpha=\frac{\log (G)}{N_{p} N_{s} L}
$$

where $N_{p}$ is the number of passes through the amplifier, $N_{s}$ is the number of slabs, and $L$ is the length of the beam path in the slab. A typical gain coefficient profile (for the $\mathrm{C}$ aperture in AMPLAB, 3-long configuration, explosion fraction 20\%) obtained from the camera images in this way is presented in Figure 4.1.

It is clear from this figure that the raw AMPLAB gain data has high frequency noise in the form of diffraction patterns and interference fringes coming from imperfections in the optics. This noise had to be removed before it could be compared to the predictions of our model. However, we discovered that standard techniques to remove it such as Fourier filtering would not work, since they also distorted the gain roll-off at the edges of the aperture. 


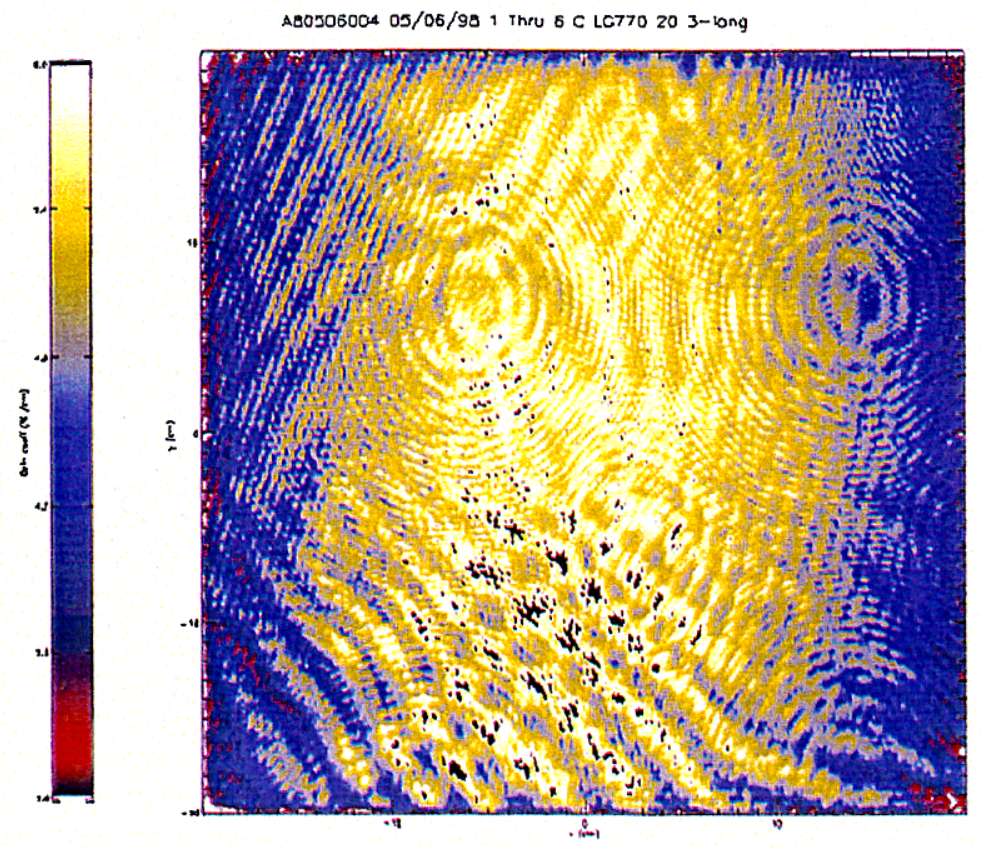

Figure 4.1. Unprocessed gain coefficient profile for AMPLAB in the 3-long configuration, $C$-aperture, $f_{x}=0.20$, calculated directly from the CCD camera images.

To solve this problem, we developed a modified boxcar-averaging technique that eliminated most of the noise without reducing the gain droop at the edges. The algorithm is illustrated schematically in Figure 4.2. The smoothed value for the gain coefficient $\left(\alpha_{i, j}\right)$ at any point was obtained by averaging over a rectangular region centered on that point:

$$
\alpha_{i, j}=\frac{1}{(2 M+1)(2 N+1)} \sum_{m=-M}^{M} \sum_{n=-N}^{N} \alpha_{i+n, j+m}
$$

In the center of the aperture, the limits $\mathrm{M}$ and $\mathrm{N}$ were fixed at a constant value $\mathrm{N}_{0}$. As an edge was approached, the corresponding limit was reduced so that we continued to average over an area centered on the point of interest. The value of the smoothing parameter $\mathrm{N} 0$ was set at 10 , (corresponding to $0.8 \%$ of the full aperture) based on a somewhat subjective examination of all of the gain data, which indicated that a lower value did not smooth the gain profile enough, and a larger value smoothed it too much. 


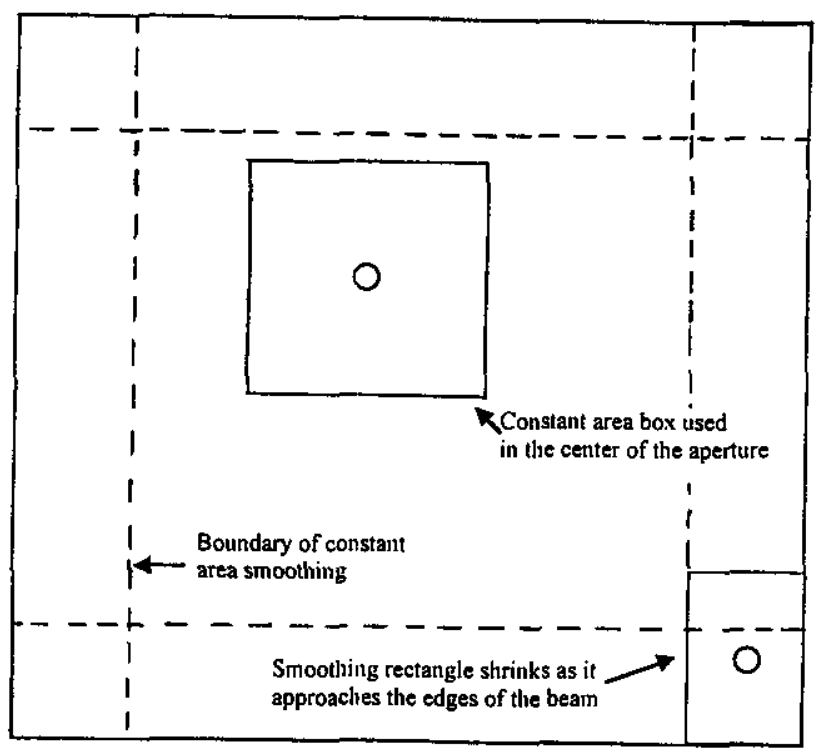

Figure 4.2. Schematic illustration of the modified boxcar-smoothing technique used to remove the high-frequency noise from the experimental gain profiles. Size of the averaging areas has been exaggerated for the purpose of illustration.

Figure 4.3 displays the smoothed version of the gain profile presented in Figure 4.1. A comparison of these two images demonstrates that our smoothing algorithm does indeed remove the noise without significantly affecting the gain roll-off at the edges of the aperture. This may be more easily illustrated in Figure 4.4, which shows the comparison between the raw and smoothed data in both horizontal and vertical line-outs.

We also obtained more accurate estimates for the experimental gain profiles in the amplifier by averaging over all of the shots that were taken for a given setup. As an example, six different measurements of the gain were taken for the $\mathrm{C}$ aperture in AMPLAB in the 3-long configuration at an explosion fraction of $20 \%$. Figure 4.5 shows the average of the smoothed gain profiles in this case. A comparison with Figure 4.3 shows that the multishot averaging further reduces the spatial noise in the gain profile. We also used this data to estimate the uncertainty in the experimental measurements by calculating a point-by-point standard deviation for the gain coefficient. The results of this analysis for the 3-long configuration are presented in Figure 4.6. The random nature of this plot suggests that a significant portion of the error may be attributable to noise that is not removed by our smoothing technique. However, the area average does give us some estimate of the overall uncertainty in the measurement, which we can use in our comparison with the model predictions. As an example, for the 3-long configuration we have discussed in this section, the overall relative error in the experimental value for the aperture-averaged gain coefficient is about $1.5 \%$. 


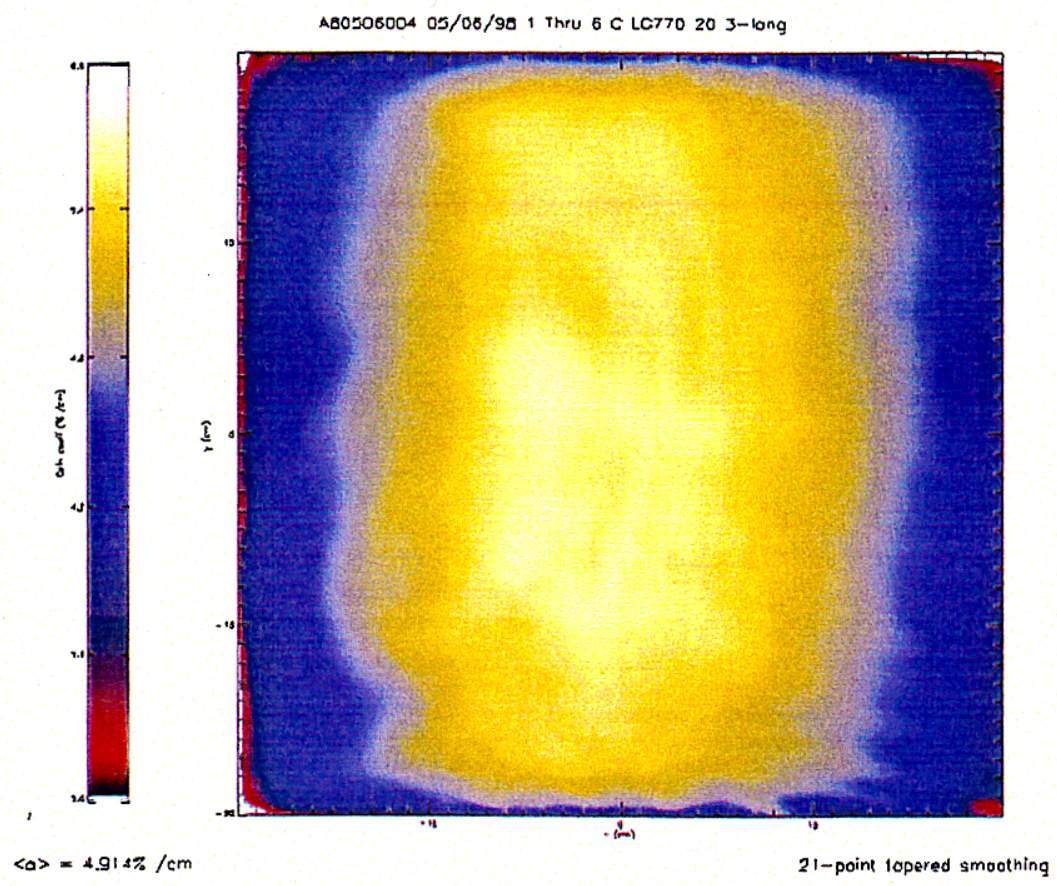

Figure 4.3. Smoothed version of the gain coefficient profile data in Figure 4.1 (AMPLAB in the 3-Iong configuration, $C$-aperture, $f_{x}=0.20$ ).
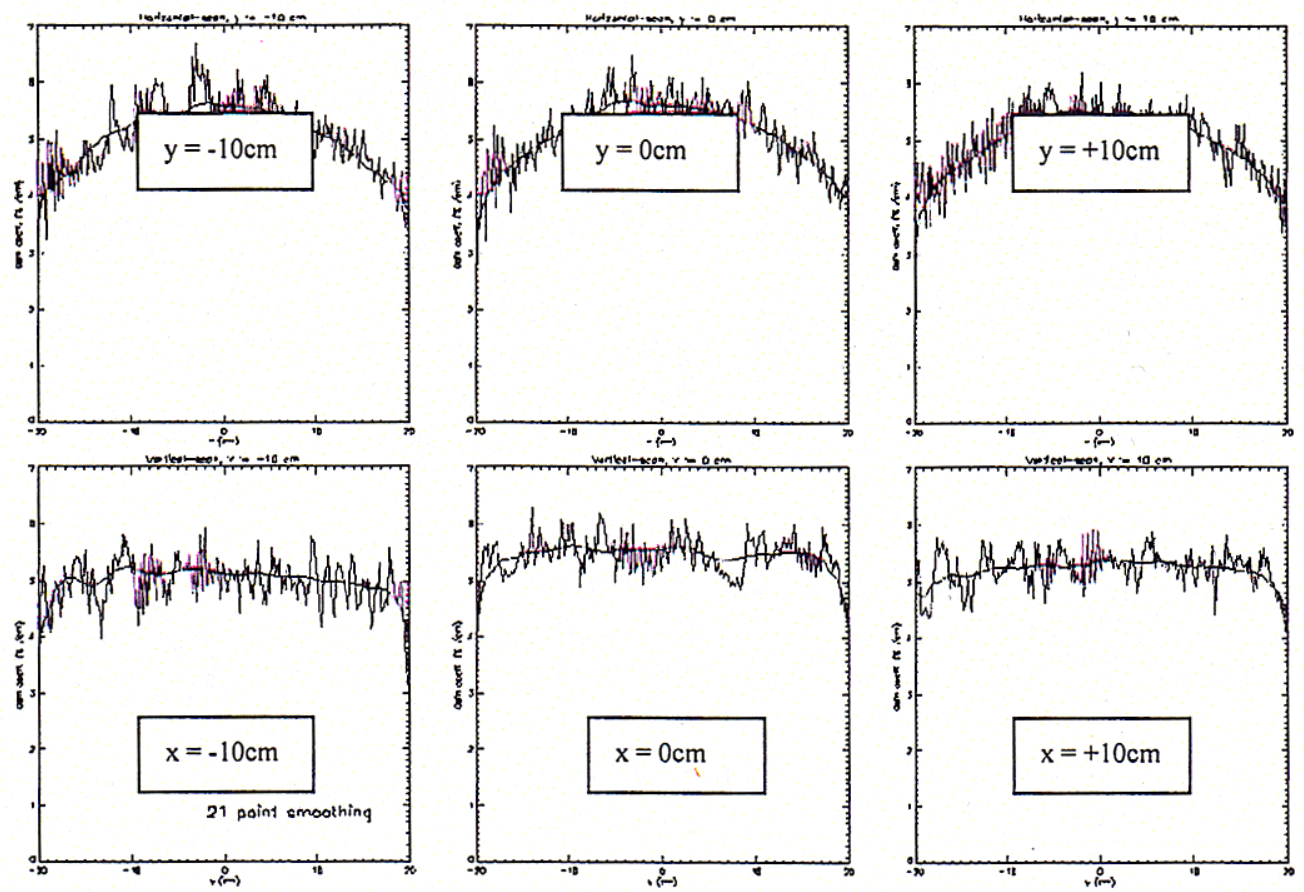

Figure 4.4. Direct comparison of the raw and smoothed gain coefficient profiles in Figures 4.1 and 4.3. Top row: horizontal line outs at $y=-10,0$, and $+10 \mathrm{~cm}$; Bottom row: Vertical line outs at $x=-$ 10,0 , and $+10 \mathrm{~cm}$. 


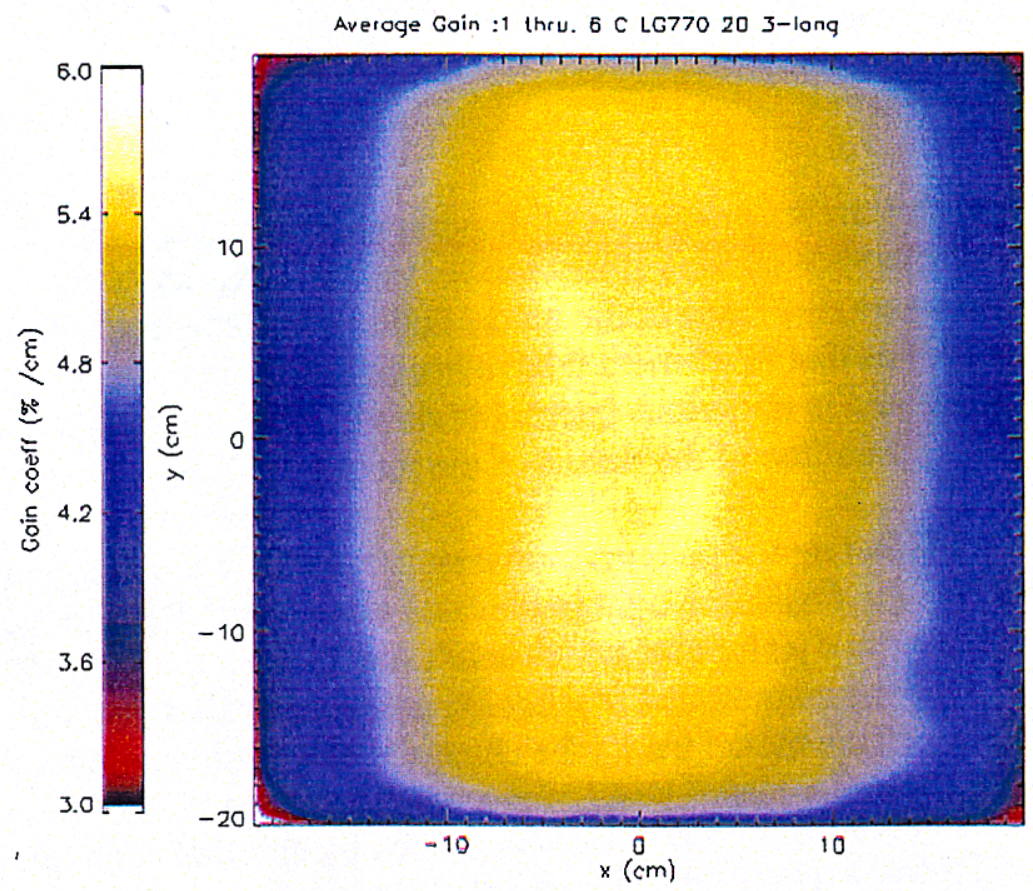

Figure 4.5. Average of six smoothed gain coefficient profiles for AMPLAB in the 3-long configuration, $C$-aperture, $f_{x}=\mathbf{0 . 2 0}$.

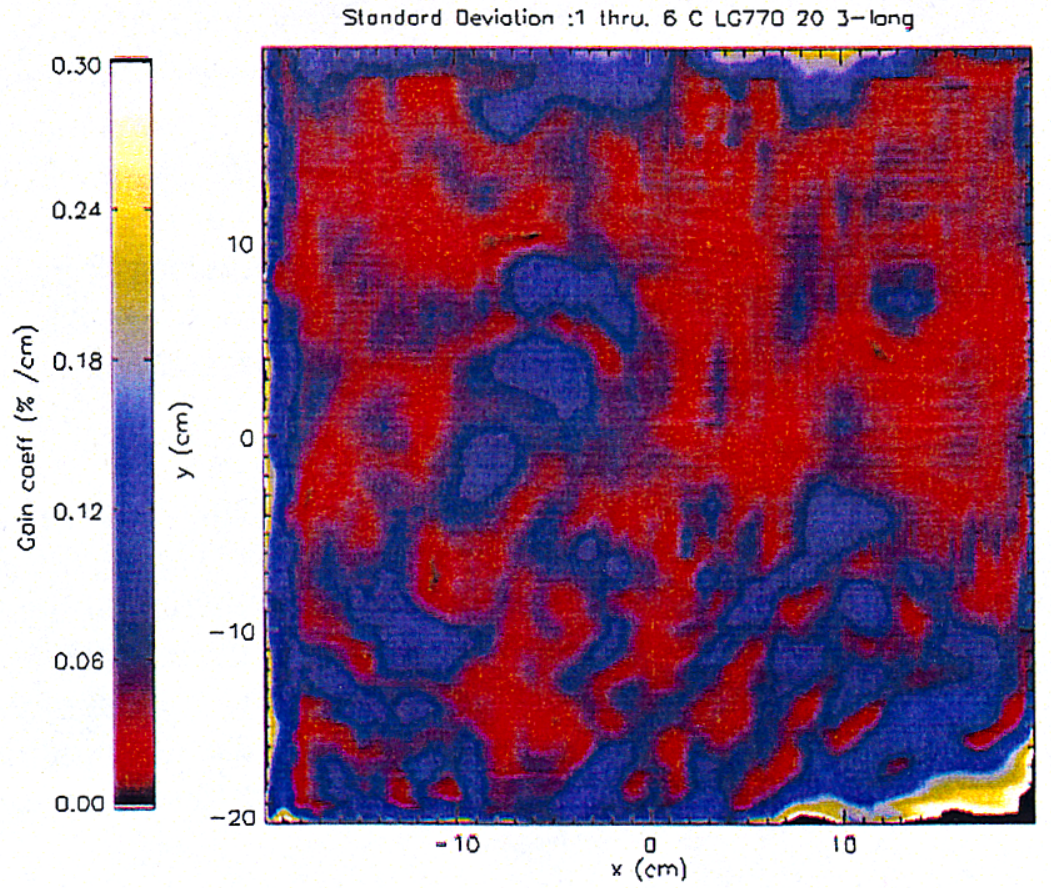

Figure 4.6. Standard deviation of six smoothed gain coefficient profiles for AMPLAB in the 3-long configuration, $C$-aperture, $\mathrm{f}_{\mathrm{x}}=\mathbf{0 . 2 0}$. 


\subsection{3-D Gain Model}

Our 3-D gain model for the prediction of the gain performance is a collaborative effort involving contributions coming from workers at both LLNL and CEA in France. Both the physical assumptions made in the model and the calculational techniques used in the computer programs that implement it have been described in detail in earlier publications [4.1,4.2], and in numerous internal reviews. Therefore, in this document we will provide only a brief outline of the details of the model.

The first step in predicting the gain performance is to calculate the pump rate of the inversion generated by the flashlamp light in the laser slabs. In our 3-D model, we do this using a reverse ray-trace technique, which sends out rays from the point of interest in the slab and determines the amount that any light sources seen by that ray contribute to the pump rate. The ray-trace model tracks the change in the spectral content of each ray as it interacts with the various surfaces and media present in the amplifier cavity. Fresnel reflection and refraction at dielectric interfaces are treated by splitting each ray into separate reflected and refracted rays when it hits a dielectric surface.

Our empirical model for the optical properties of xenon flashlamps is used to predict both the emission and absorption of the xenon plasma [4.3]. The model uses experimentally determined values for the absorption and reflectance of all of cavity components, including laser slabs, reflectors, and blastshields.

The latest version of the 3-D code also models the detailed spectral effects of a single-layer AR-coating on glass surfaces. However, preliminary calculations by the French for the AMPLAB geometry indicate that the only consequences of implementing this feature are that the overall gain coefficient goes up by about $5 \%$ (consistent with our experimental observations of the gain seen with and without AR-coatings on blastshields) with no significant change in the profile, while the run-time goes up by a factor of 3 to 4 . For these reasons, we chose not to implement this feature for the calculations in this report due to time constraints. Instead, we accounted for the presence of the AR-coating on the blastshields by multiplying the predicted gain profiles for uncoated blastshields by a factor of 1.05 .

Once the pump distribution in the slab as a function of the flashlamp loading has been determined, we calculate the peak gain coefficient by numerically solving the differential equation for the stored energy density at each point in the slab as a function of time. In addition to radiative and nonradiative spontaneous decay processes, the model also tracks the spatially and temporally dependent ASE decay rate throughout the volume of the laser slabs. To accurately calculate this ASE decay rate, we have to use a nonuniform grid of points through the thickness of the slab, with more points near the surface where the gain coefficient and the ASE decay rate is the highest.

All of the model calculations are carried out on a dedicated cluster of 28 Unix workstations. The computational load is distributed over the cluster using a public domain package (PVM) for networked parallel computing. A typical calculation of the 
pump rates for a grid containing 4950 points ( 30 horizontal $\times 15$ vertical $\times 11$ deep) to a computational accuracy of about $1 \%$ takes about 12 hours of computing time on the cluster. The calculation of the peak gain coefficient distribution takes an additional six hours to complete.

\subsection{Comparison of gain measurements to the 3D model predictions}

Gain profiles have been obtained in AMPLAB over a broad range of amplifier configurations, as illustrated in Table 4.1. Gain measurements were performed in the four apertures on one side of the central flashlamp array, which were labeled A, B, C, and $\mathrm{D}$, from top to bottom. While the gain was not measured for every possible configuration, there is sufficient data to answer the questions required to validate the 3-D model for use in predicting the gain performance of the NIF amplifier. Before we begin with the comparisons of the gain measurements to the model predictions, we will use the experimental data to check two important assumptions about the amplifier physics. We will then proceed with a set of comparisons to demonstrate the ability of the 3-D model to predict the gain profile for all the slab positions, apertures, glass types, and explosion fractions of interest. 
Table 4.1. Summary of AMPLAB gain measurements and modeling comparisons.

\begin{tabular}{|c|c|c|c|c|c|c|}
\hline $\begin{array}{l}\text { flashiamp } \\
\text { explosion } \\
\text { fraction }\end{array}$ & Configuration & Aperture & Gain file name & $\begin{array}{l}\text { Measured aperture- } \\
\text { averaged gain } \\
\text { coefficient }(\% / \mathrm{cm})\end{array}$ & $\begin{array}{l}\text { Predicted aperture- } \\
\text { averaged gain } \\
\text { coefficient }(\% / \mathrm{cm})\end{array}$ & $\begin{array}{l}\text { Reiated } \\
\text { figures }\end{array}$ \\
\hline 0.15 & $\begin{array}{l}\text { X-two slabs } \\
\text { wide }\end{array}$ & $D$ & A80219012 & 4.38 & 4.15 & $4.36,4.37$ \\
\hline 0.2 & $\begin{array}{l}\text { Diamond - } \\
\text { two slabs wide }\end{array}$ & A & $\begin{array}{l}\text { A80209004, } \\
\text { A80209010 }\end{array}$ & 4.43 & 4.41 & $4.33,4.34$ \\
\hline & & D & $\begin{array}{l}\text { A80113003, } \\
\text { A80113005 }\end{array}$ & 4.73 & 4.62 & $4.27,4.28$ \\
\hline & $\begin{array}{l}\text { Diamond - } \\
\text { one slab wide }\end{array}$ & C & $\begin{array}{l}\text { A80507002, } \\
\text { A80507004, } \\
\text { A80507008 }\end{array}$ & 4.73 & 4.77 & $4.19,4.20$ \\
\hline & $\begin{array}{l}X \text { - two slabs } \\
\text { wide }\end{array}$ & A & $\begin{array}{l}\text { A80219002, } \\
\text { A80219008 }\end{array}$ & 4.52 & 4.48 & $4.30,4.31$ \\
\hline & & B & $\begin{array}{l}\text { A80220002, } \\
\text { A80220004 (?) }\end{array}$ & 4.83 & & 4.12 \\
\hline & & C & $\begin{array}{l}\text { A80223002, } \\
\text { A80223008 (?) }\end{array}$ & 4.8 & & 4.11 \\
\hline & & D & $\begin{array}{l}\text { A80224003, } \\
\text { A80303002, } \\
\text { A80304002, } \\
\text { A80305002, } \\
A 80306002, \\
A 8031004, \\
A 80317002\end{array}$ & 4.68 & 4.8 & $4.7,4.25$ \\
\hline & $\begin{array}{l}X \text { - one-slab } \\
\text { wide }\end{array}$ & C & $\begin{array}{l}A 80406002, \\
\text { A80406004, } \\
\text { A80406006, } \\
\text { A80406008, } \\
\text { A80508002 }\end{array}$ & 4.7 & 4.8 & $4.15,4.16$ \\
\hline & & $D$ & $\begin{array}{l}\text { A80319002, } \\
\text { A80319004, } \\
\text { A80319006 }\end{array}$ & 4.7 & 4.8 & $4.8,4.25$ \\
\hline & 3-slab-long & C & $\begin{array}{l}A 80505002, \\
A 80506002, \\
A 80506004, \\
A 80515002, \\
A 80519003 \\
A 80521006\end{array}$ & & & 4.5 \\
\hline & internal slab & C & $\begin{array}{l}\text { combinations } \\
\text { of the above }\end{array}$ & 5.33 & 5.27 & $4.22,4.23$ \\
\hline 0.23 & $\begin{array}{l}\text { X-two slabs } \\
\text { wide }\end{array}$ & D & $\begin{array}{l}\text { A80224005 } \\
\text { A80224011 }\end{array}$ & 4.88 & 4.93 & $439,4.40$ \\
\hline
\end{tabular}

\subsubsection{Validation of the assumptions made in building the amplifier model}

Two assumptions about the amplifier physics were built into the 3-D model. The model assumes first that the amount of pump light that leaks through the central array from one side of the amplifier to the other is negligible. The second assumption is that the horizontal centerline is a reflection plane of symmetry for the pumping profiles. 
We can test the first assumption by looking at a comparison of one-wide vs. two-wide gain measurements. Figure 4.7 shows the smoothed and averaged gain profile for the $D$ aperture in the two-wide $X$-configuration at a flashlamp explosion fraction of $20 \%$, which had an aperture-averaged gain coeffiecent, $\langle\propto\rangle$, of $4.68 \% / \mathrm{cm}$. Figure 4.8 shows the smoothed and averaged gain profile for the corresponding one-wide configuration for which $\langle\propto\rangle$ was $4.7 \% / \mathrm{cm}$, about $0.5 \%$ greater than the two-wide average. Figure 4.9 shows the absolute value of the difference between these two profiles, which show no systematic difference between the two distributions. Figure 4.10 gives the point-by-point value of the standard deviation for the average profile for the two-wide, for which $\langle\Delta \propto\rangle$ was $0.18 \% / \mathrm{cm}$, about 9 times greater than the difference between one- and two-wide gain distribution. The random nature of the difference between the two gain profiles and the fact that its magnitude is less than the error in just one of them implies that they are equal to within the experimental uncertainty, validating our first assumption.

We can test our second assumption, symmetry about the horizontal centerline, by comparing the gain profile in the $\mathrm{C}$ aperture to a flipped version of that in the $\mathrm{B}$ aperture. Figure 4.11 shows the average gain profile for the $\mathrm{C}$ aperture in the 2 -wide $\mathrm{X}$ configuration at an explosion fraction of $20 \%$, for which $\langle\propto\rangle$ was $4.80 \% / \mathrm{cm}$. Figure 4.12 gives the corresponding average gain profile for the $\mathrm{B}$ aperture flipped about the $x$-axis, for which $\langle\propto\rangle$ was $4.83 \% / \mathrm{cm}$. Figure 4.13 shows the absolute value of the difference between the $\mathrm{C}$ aperture profile and the flipped $\mathrm{B}$ aperture, which has a random distribution. Figure 4.14 shows the point-by-point value of the standard deviation for the average profile for the flipped $B$ aperture, which has an aperture-averaged value of $0.15 \% / \mathrm{cm}$, about 5 times greater than the difference between the aperture B and C. Again, the random nature of the difference between the two gain profiles and the fact that its magnitude is slightly less than the error in just one of them implies that they are equal to within the experimental uncertainty, validating our second assumption.

\subsubsection{Comparison of the measurements and model predictions for both end and interior slabs}

\section{$X$ Configuration ( $C$ aperture)}

We begin with the gain profile in the $\mathrm{C}$ aperture in the one-wide $\mathrm{X}$-configuration at an explosion fraction of $20 \%$. Smoothing and averaging the experimental data produces the gain profile shown in Figure 4.15. It is important to note that this level of performance, with $\langle\propto\rangle=4.80 \% \mathrm{~cm}$, was obtained with slightly tarnished silver reflectors that had an average reflectance of $\sim 91 \%$ at $670 \mathrm{~nm}$, compared with $\sim 96 \%$ for pristine silver. This degradation factor was used in all of the 3-D modeling simulations for AMPLAB. 


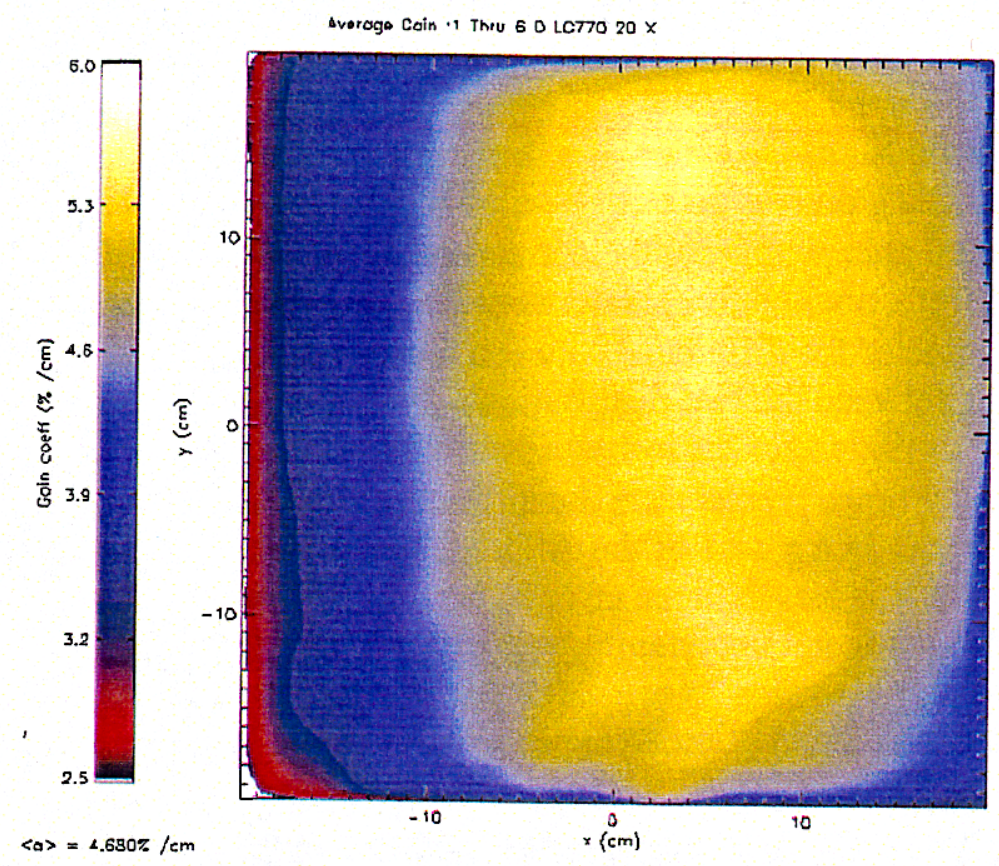

Figure 4.7. Smoothed and averaged gain profile for the $\mathrm{D}$ aperture in the two-wide $\mathrm{X}$-configuration at a flashlamp explosion fraction of $20 \%$.

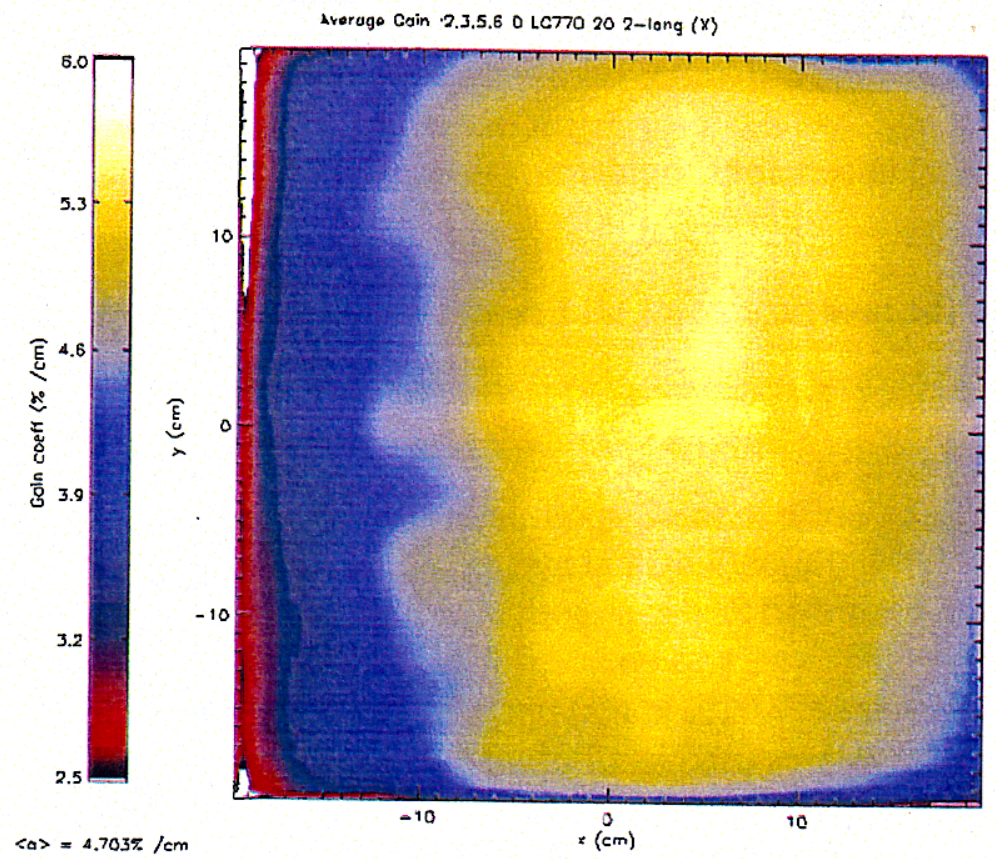

Figure 4.8. Smoothed and averaged gain profile for the $D$ aperture in the 1-wide, $X$-configuration at a flashlamp explosion fraction of $20 \%$. 


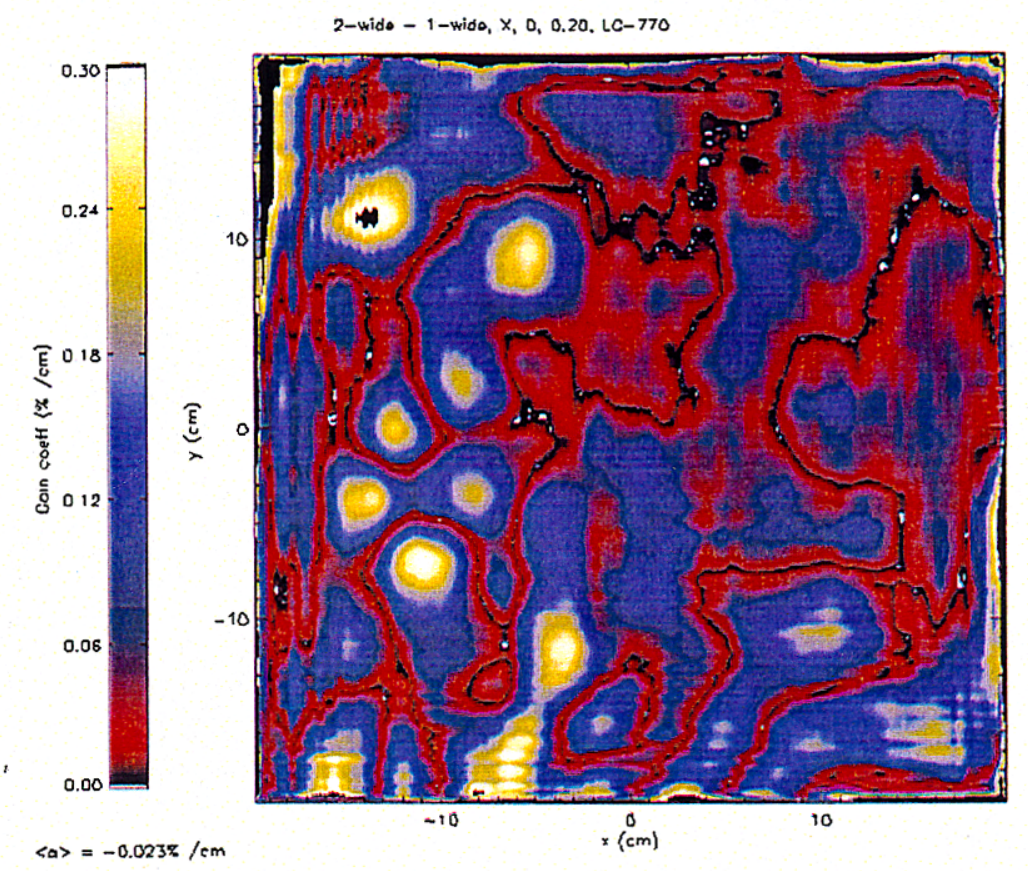

Figure 4.9. Absolute value of the difference between the two-Wide and one-Wide gain profiles in the $\mathrm{D}$ aperture in the X-configuration at a flashlamp explosion fraction of $20 \%$.

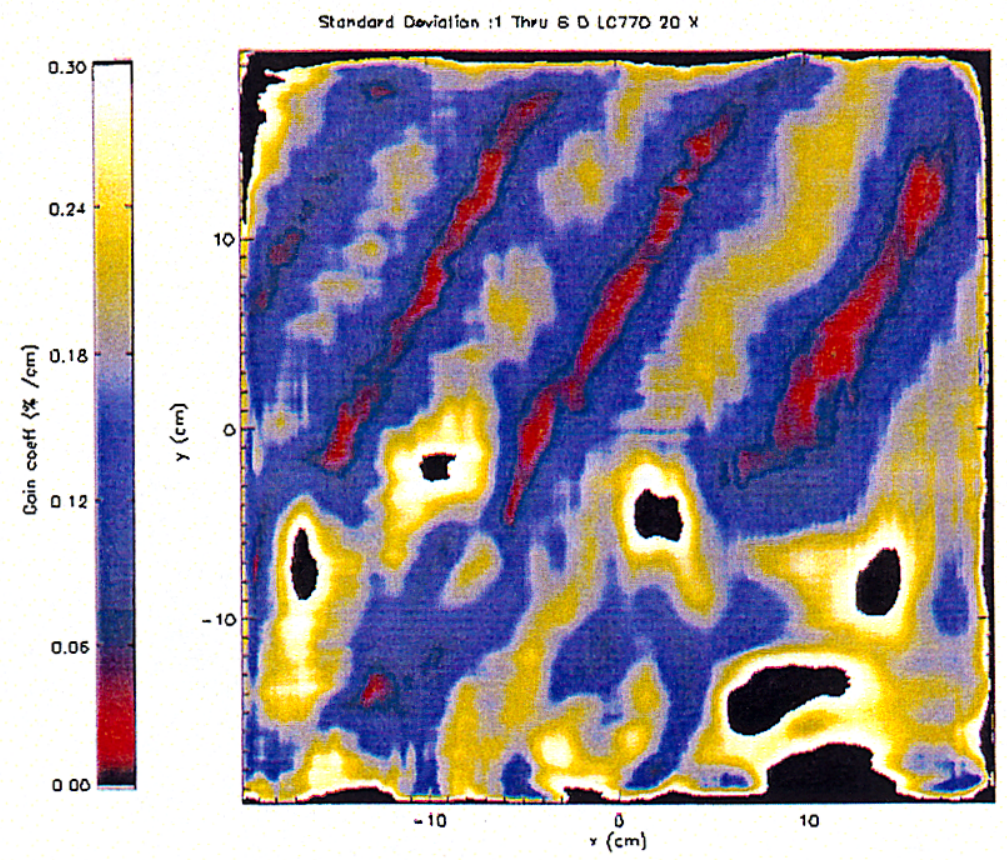

Figure 4.10. Point-by point standard deviation for the averaged gain profile in the $D$ aperture in the two-wide X-configuration at a flashlamp explosion fraction of $20 \%$. 


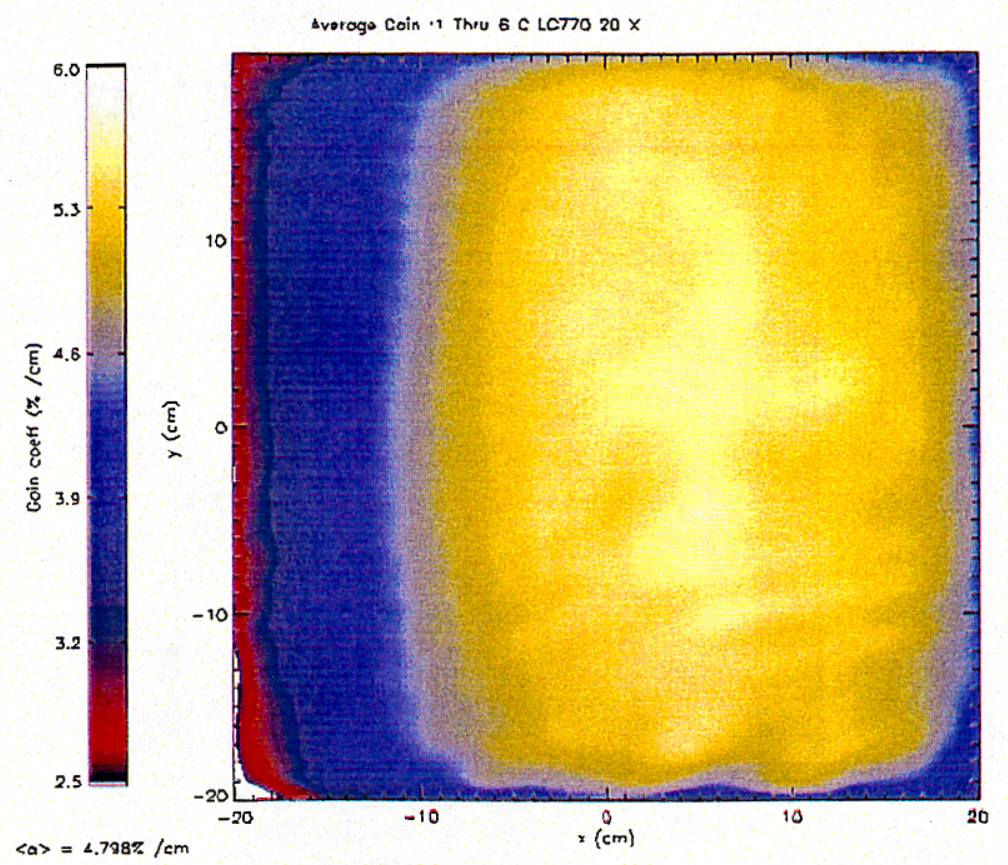

Figure 4.11. Smoothed and averaged gain profile for the $\mathrm{C}$ aperture in the two-wide $\mathrm{X}$-configuration at a flashlamp explosion fraction of $20 \%$.

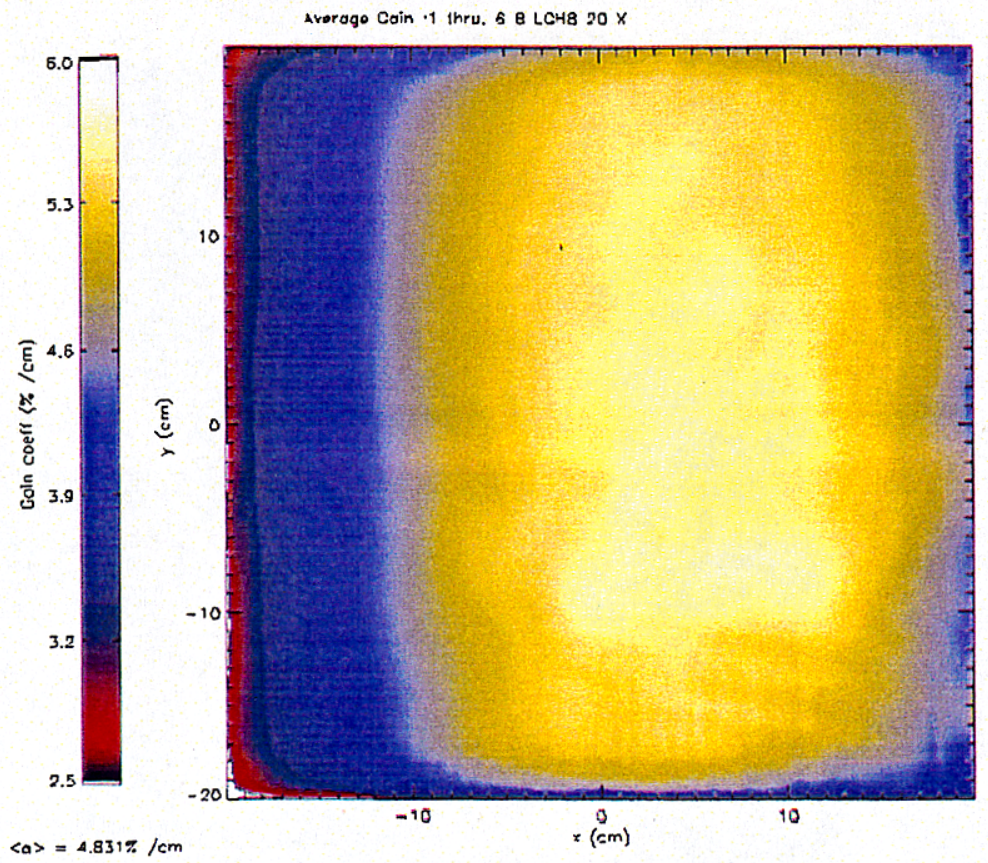

Figure 4.12. Smoothed and averaged gain profile for the $B$ aperture in the two-wide $X$-configuration at a flashlamp explosion fraction of $20 \%$, flipped about the $x$-axis. 


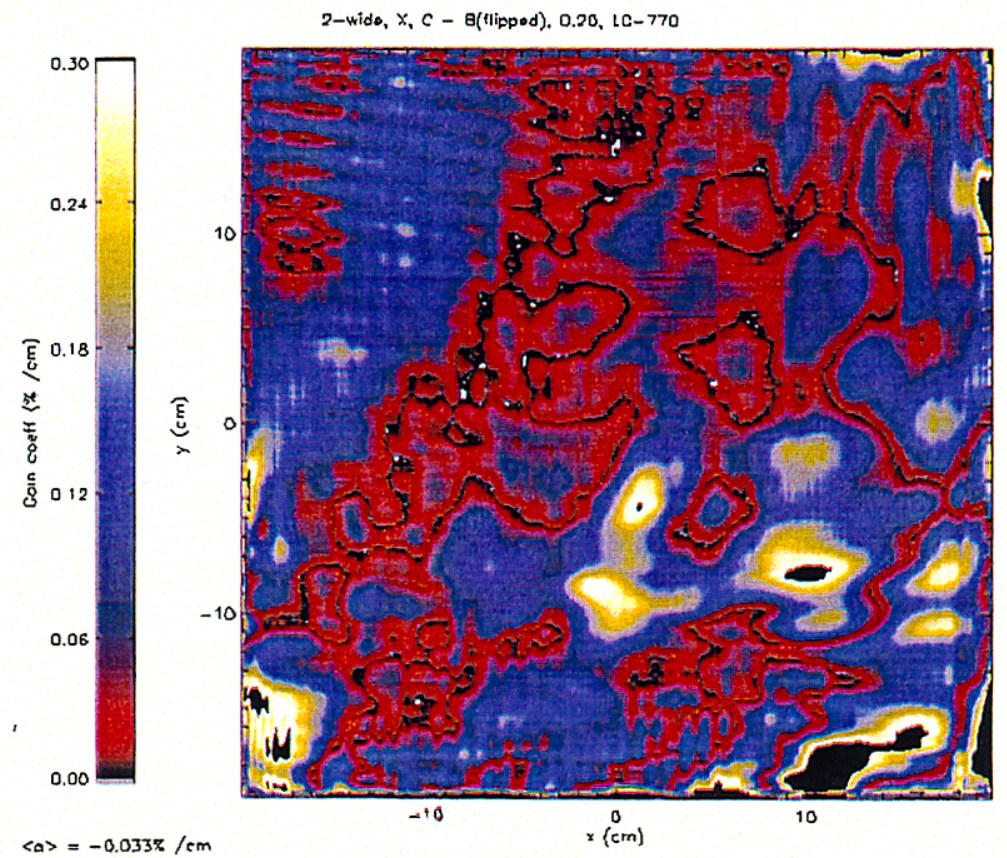

Figure 4.13. Absolute value of the difference between the $C$ and flipped $B$ aperture gain profiles in the X-configuration at a flashlamp explosion fraction of $20 \%$.

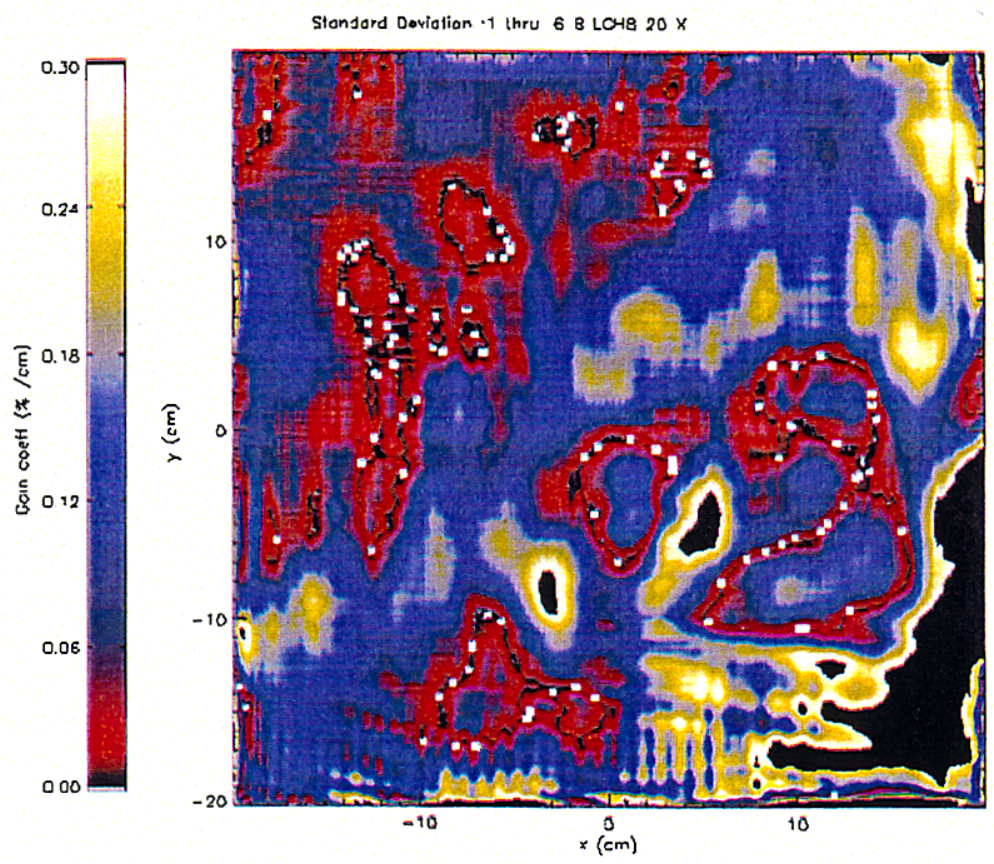

Figure 4.14. Point-by point standard deviation for the flipped averaged gain profile in the $B$ aperture in the two-wide $\mathrm{X}$-configuration at a flashlamp explosion fraction of $20 \%$. 
The 3-D model prediction for the gain profile for this configuration is presented in Figure 4.16. The predicted value of $\langle\propto\rangle$ was $4.70 \% / \mathrm{cm}, \sim 2 \%$ lower than the measured value. In comparing the measured and predicted gain profiles in this case and those that follow, it is important to note that the model plots are restricted to a range of $\pm 18.5 \mathrm{~cm}$, so they do not show any indication of the measured roll-off at the top and bottom of the aperture." We can get a better indication of the agreement between the measured and predicted profiles by looking at line-out comparisons, presented in Figure 4.17. For comparison, Figure 4.18 gives the standard deviation, $\langle\Delta \propto\rangle$, in the experimental measurement, which was $0.06 \% / \mathrm{cm}$ (relative error of $1.3 \%$ ). We see that for this case, the model agrees with the experiment to within the $\sim \pm 2 \%$ experimental over most of the aperture, but it slightly under-estimates the roll-off at the edges of the beam, where the relative local difference between the two curves rises to $6 \%$.

\section{Diamond configuration ( $\mathrm{C}$ aperture)}

A similar comparison between the measured and predicted gain for the $\mathrm{C}$ aperture in the diamond configuration is presented in Figures 4.19 through 4.21. Again, the measurement and model predictions agree to within the experimental error $( \pm 1.5 \%$ relative error, averaged over the entire profile) over most of the aperture, but there are local regions where the difference rises to as much as $6 \%$.

\section{Interior slabs ( $D$ aperture)}

We can derive an experimental estimate for the gain profile in a interior slab $\left(\alpha_{i}\right)$ in the $\mathrm{D}$ aperture by combining the average gain profiles for the X-configuration $\left(\alpha_{X}\right)$ and diamond-configuration $\left(\alpha_{d}\right)$ with that of the 3-long configuration $\left(\alpha_{3}\right)$ derived in Section 4.1 above using the relationship:

$$
\alpha_{i}=3 \alpha_{3}-\alpha_{x}-\alpha_{d}
$$

The results of this computation are presented in Figure 4.22. The model prediction for a central slab in the D aperture is presented in Figure 4.23, and line-out comparisons of the two profiles are plotted in Figure 4.24. The relative error in the experimental profile is $0.04 \% / \mathrm{cm}$, about 3 times larger than it is in the directly observed profiles as a consequence of the manipulation of equation 4.4. There is good agreement between the model and experiment over most of the aperture; in a few small regions the relative difference rises to about $8 \%$, some of which can be directly attributed to noise in the experimental signal.

\footnotetext{
"This is not a limitation of the model, but a result of the choice of grid coordinates chosen during the initial modeling runs for this report. Later runs made with the full $20-\mathrm{cm}$ coordinate range do not show the rolloff at the top and bottom of the aperture, however.
} 


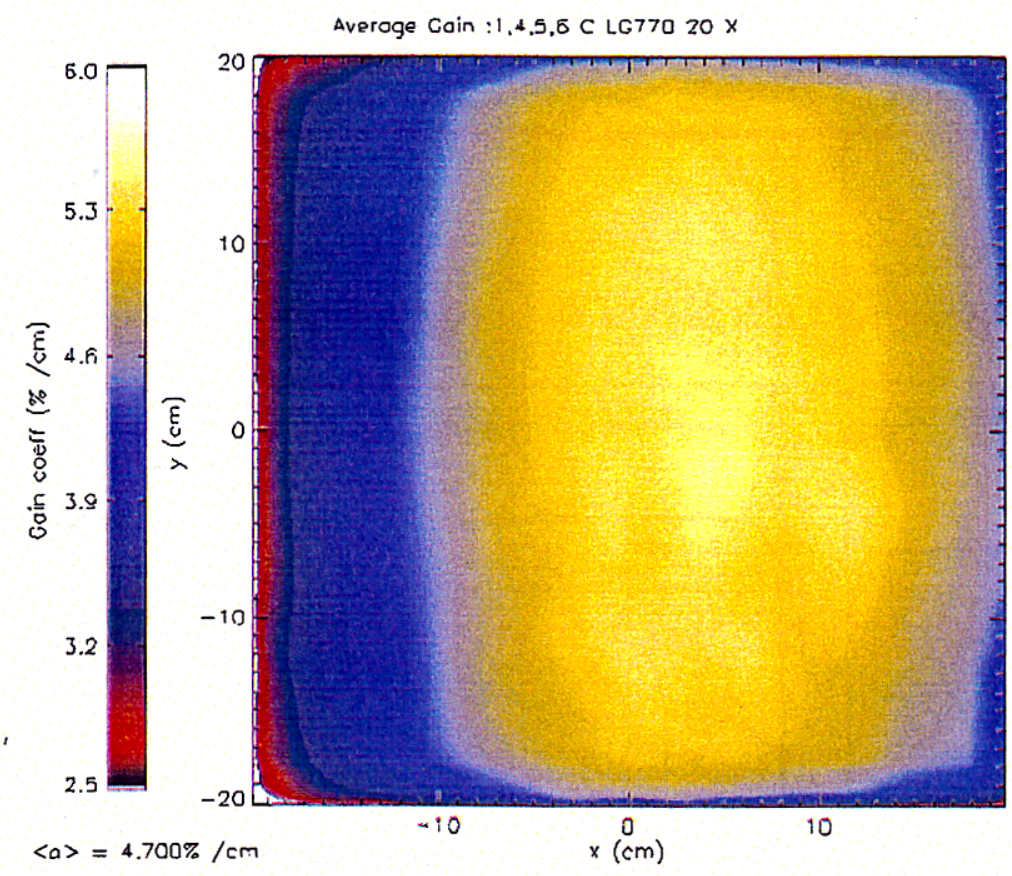

Figure 4.15. Smoothed and averaged gain profile in the $\mathrm{C}$ aperture in the one-wide $\mathrm{X}$-configuration at a flashlamp explosion fraction of $20 \%$.

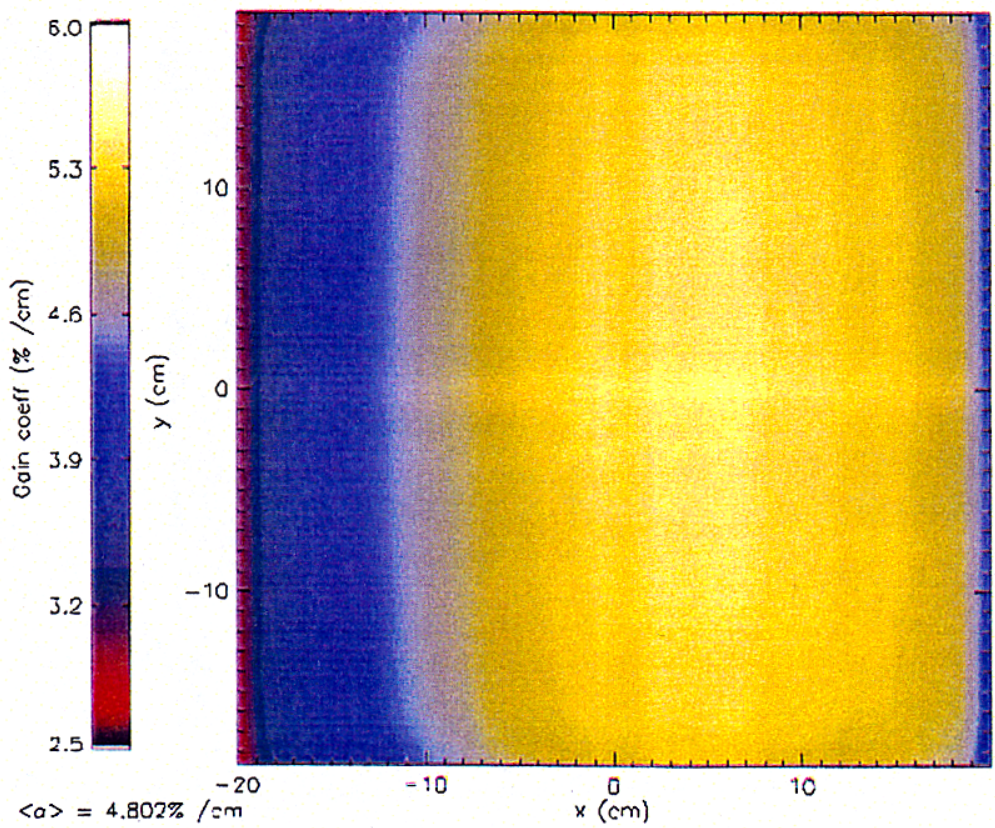

Figure 4.16. 3-D model prediction for the gain profile for the $\mathrm{C}$ aperture in the $\mathrm{X}$-configuration at a flashlamp explosion fraction of $20 \%$ (N.B.: $y$-axis limits in this plot are $\pm 18.5 \mathrm{~cm}$ ). 

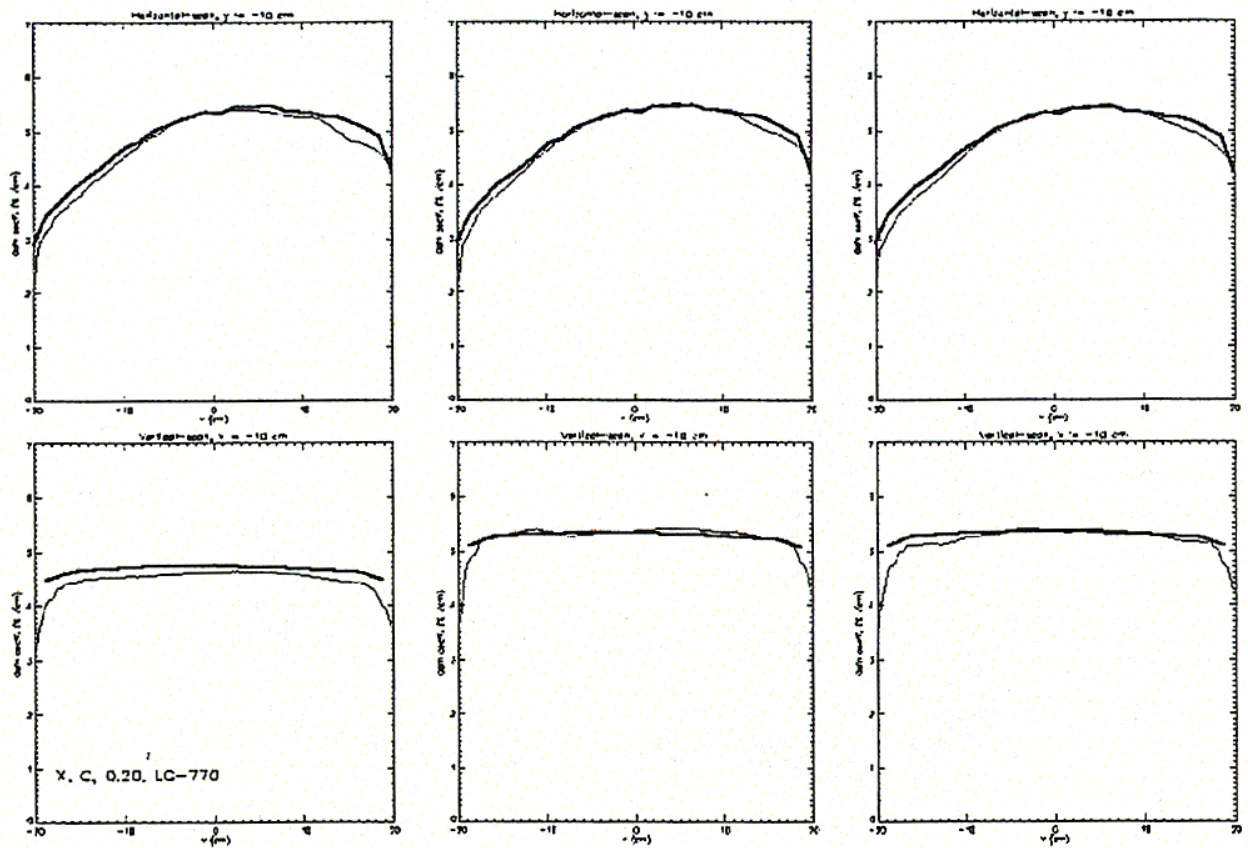

Figure 4.17. Line-out comparisons of the measured (light line) and predicted (bold line) gaincoefficient profiles for the $\mathbf{C}$ aperture in the $\mathrm{X}$ configuration, for which full-aperture gain

distributions are presented in Figures 4.16 and 4.17. Top row: horizontal line-outs at $y=-10,0$, and $+10 \mathrm{~cm}$; Bottom row: Vertical line-outs at $x=-10,0$ and $+10 \mathrm{~cm}$.

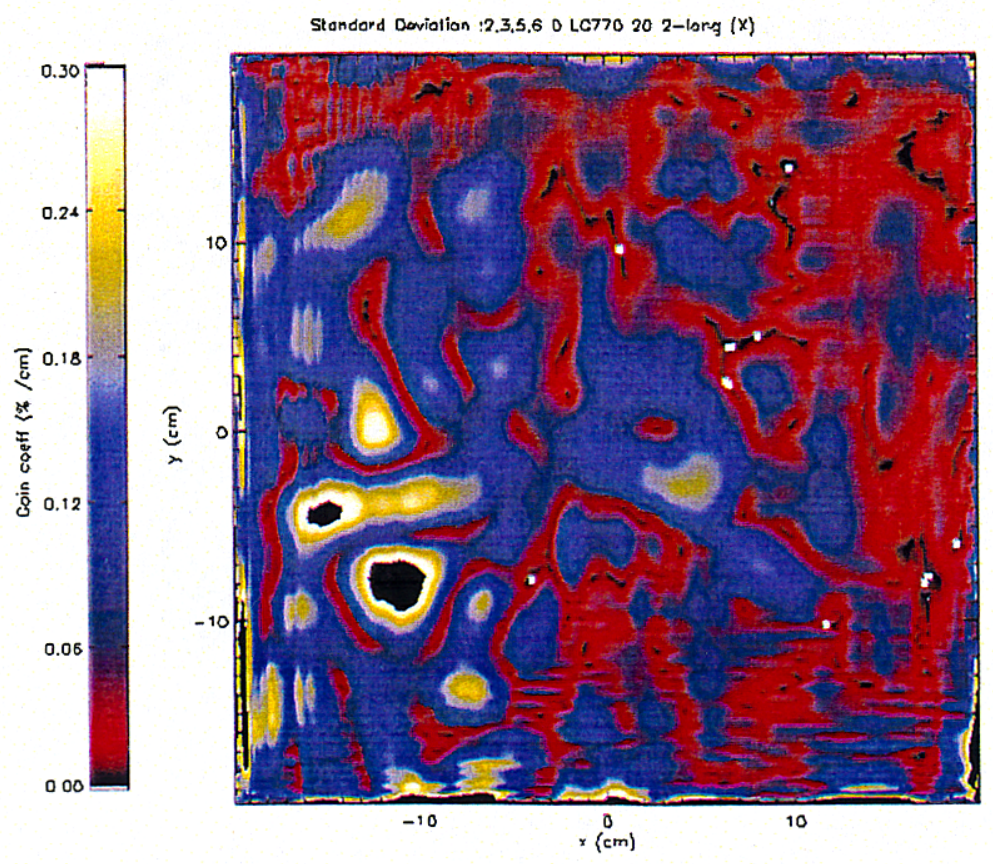

Figure 4.18. Point-by-point standard deviation for the average gain profile in the $\mathrm{C}$ aperture in the one-wide X-configuration at a flashlamp explosion fraction of $20 \%$. 


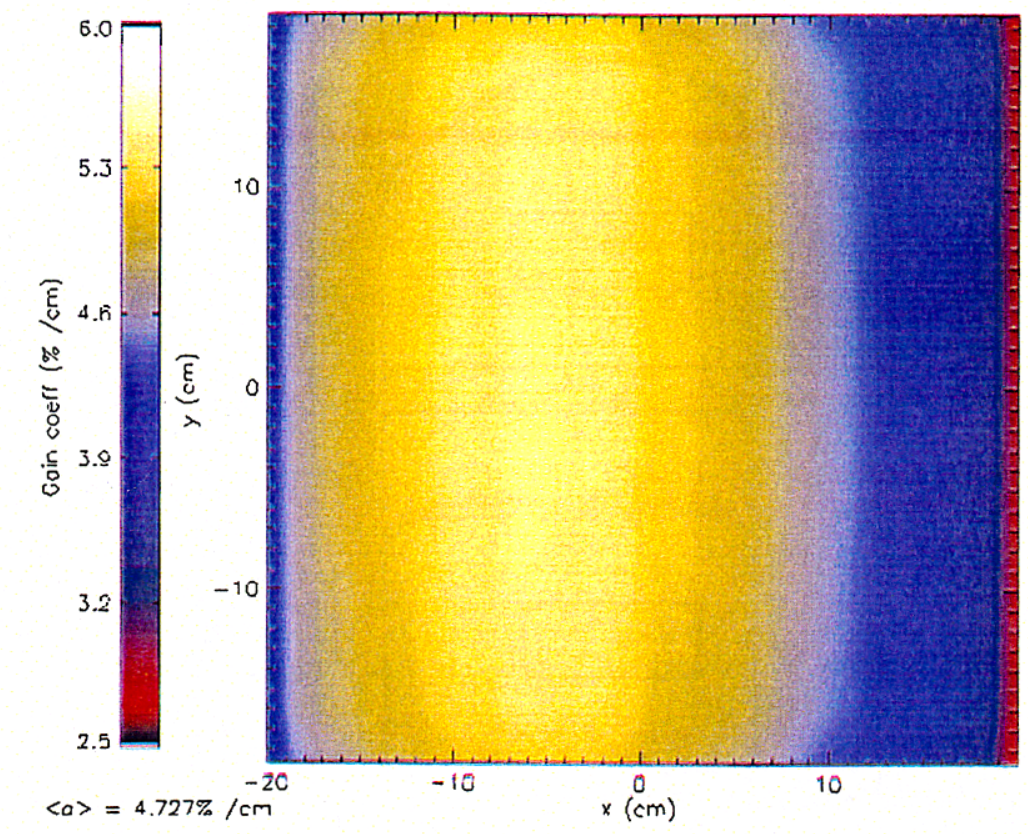

Figure 4.19. Smoothed and averaged gain profile in the $\mathrm{C}$ aperture in the one-wide diamond configuration at a flashlamp explosion fraction of $20 \%$.

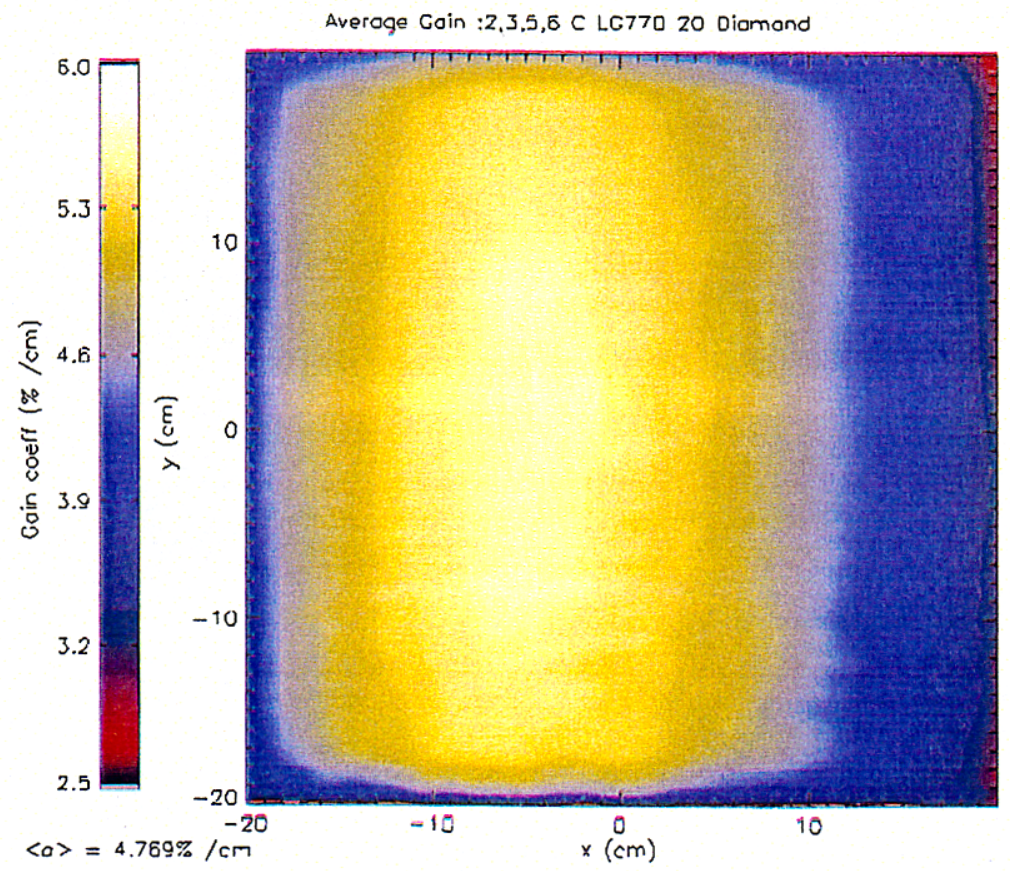

Figure 4.20.3-D model prediction for the gain profile for the $C$ aperture in the diamond configuration at a flashlamp explosion fraction of $20 \%$ (N.B.: $y$-axis limits in this plot are $\pm 18.5 \mathrm{~cm}$ ). 

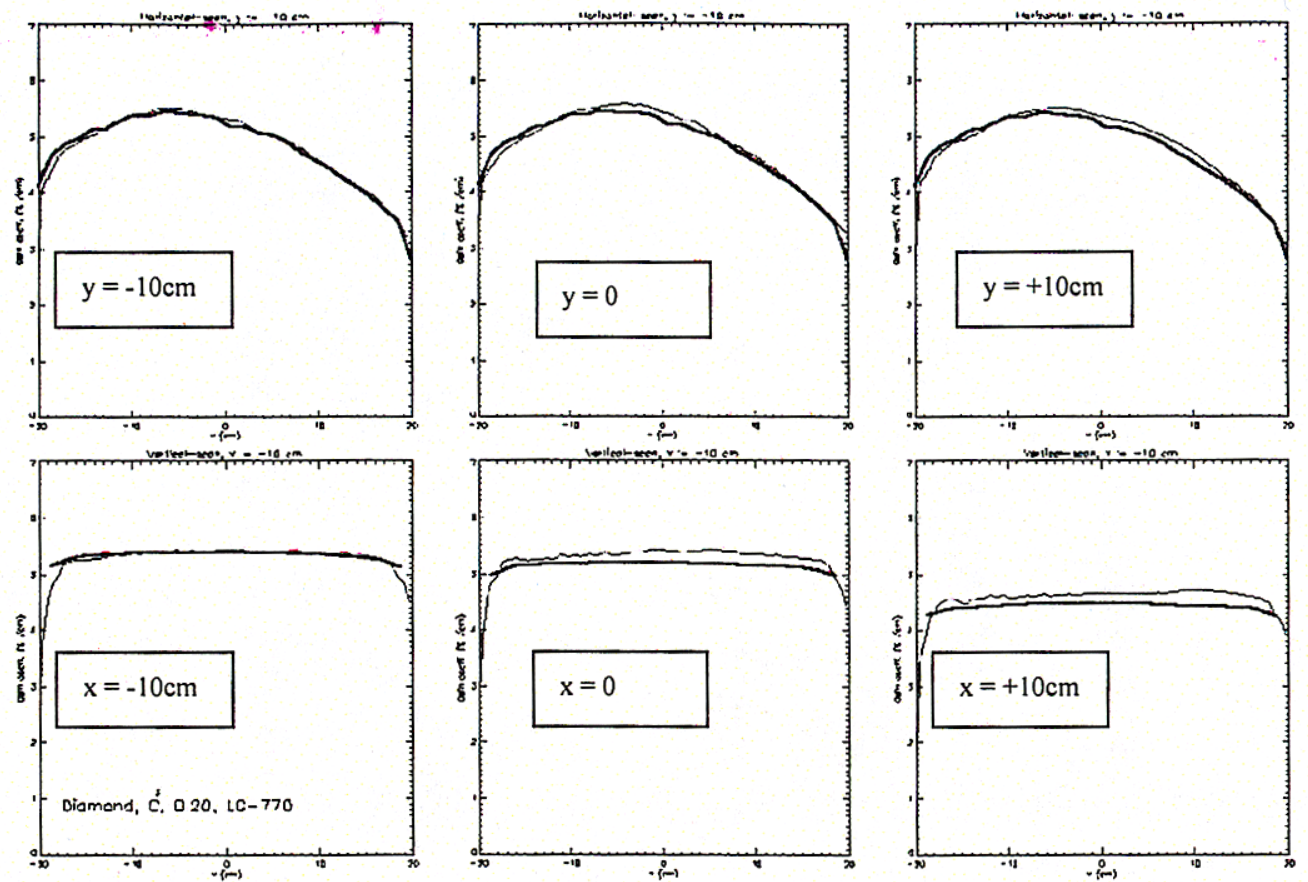

Figure 4.21. Line-out comparisons of the measured (light line) and predicted (bold line) gain coefficient profiles for the $\mathrm{C}$ aperture in the $\mathrm{X}$ configuration, for which full-aperture gain distribution are presented in Figures 4.20 and 4.21. Top row: horizontal line-outs at $y=-10,0$, and $+10 \mathrm{~cm}$; Bottom row: Vertical line-outs at $x=-10,0$ and $+10 \mathrm{~cm}$.

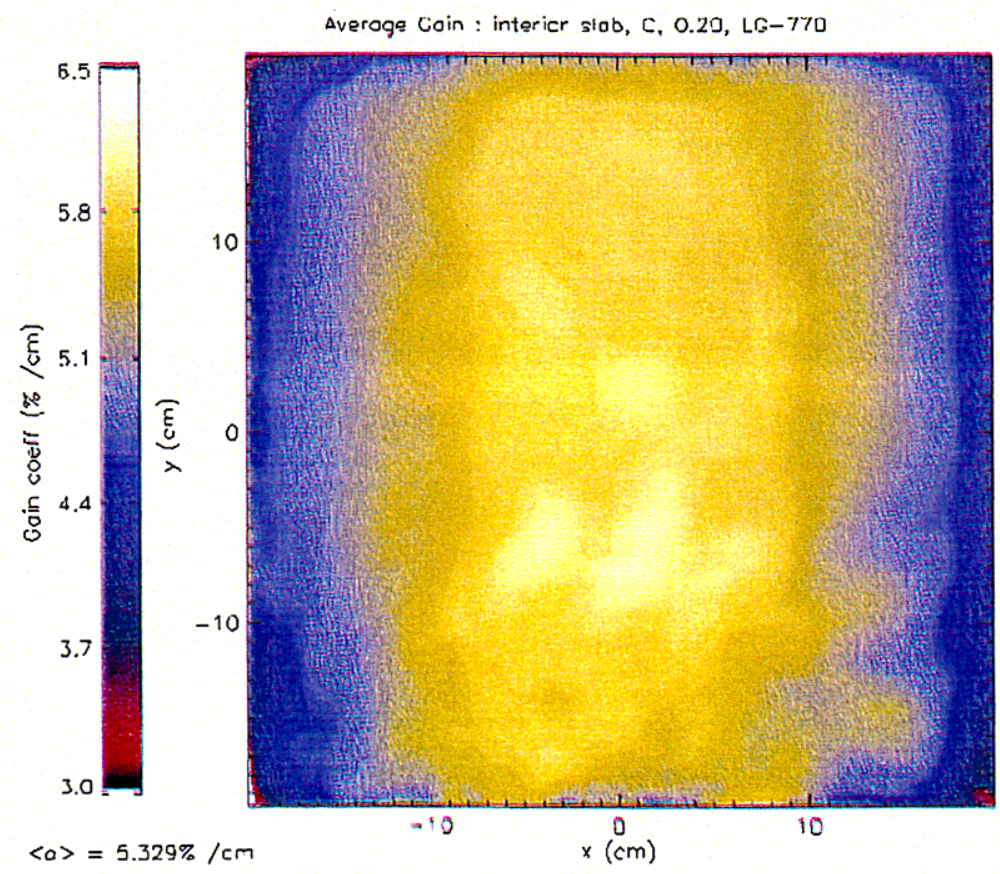

Figure 4.22. Experimental estimate for the gain profile in the $\mathrm{C}$ aperture of the interior slab for the one-wide configuration at a flashlamp explosion fraction of $20 \%$. 


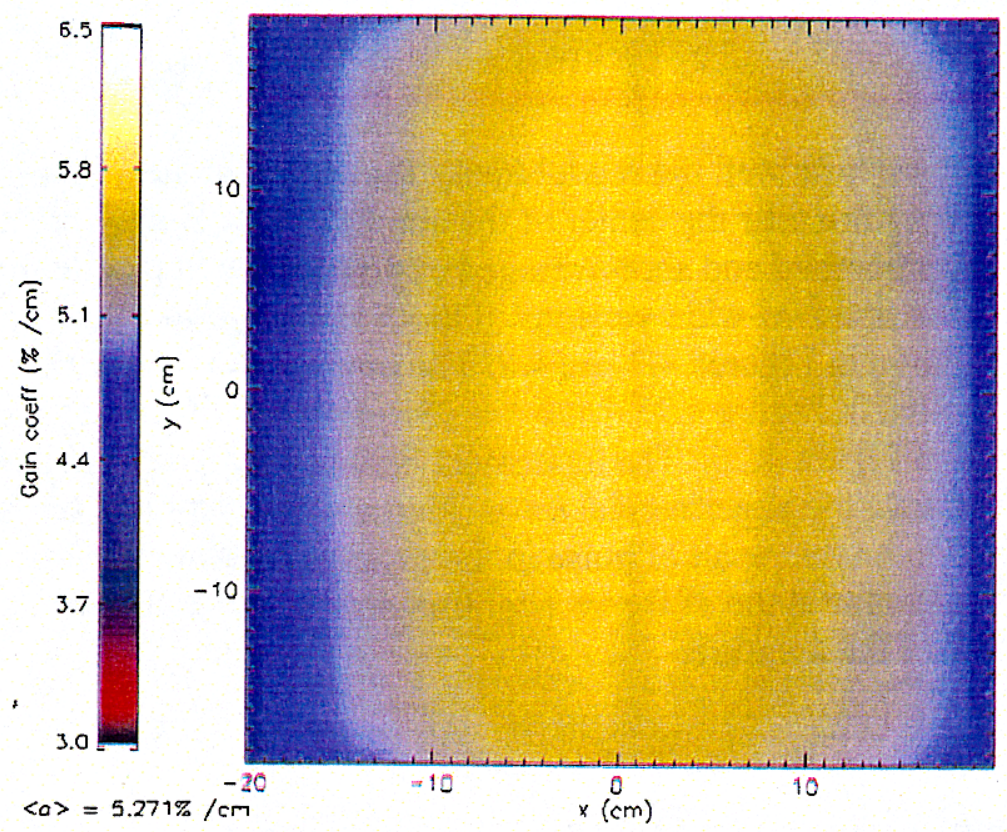

Figure 4.23. 3-D model prediction for the gain profile for the $\mathrm{C}$ aperture in an interior slab at a flashlamp explosion fraction of $20 \%$ (N.B.: $y$-axis limits in this plot are $\pm 18.5 \mathrm{~cm}$ ).
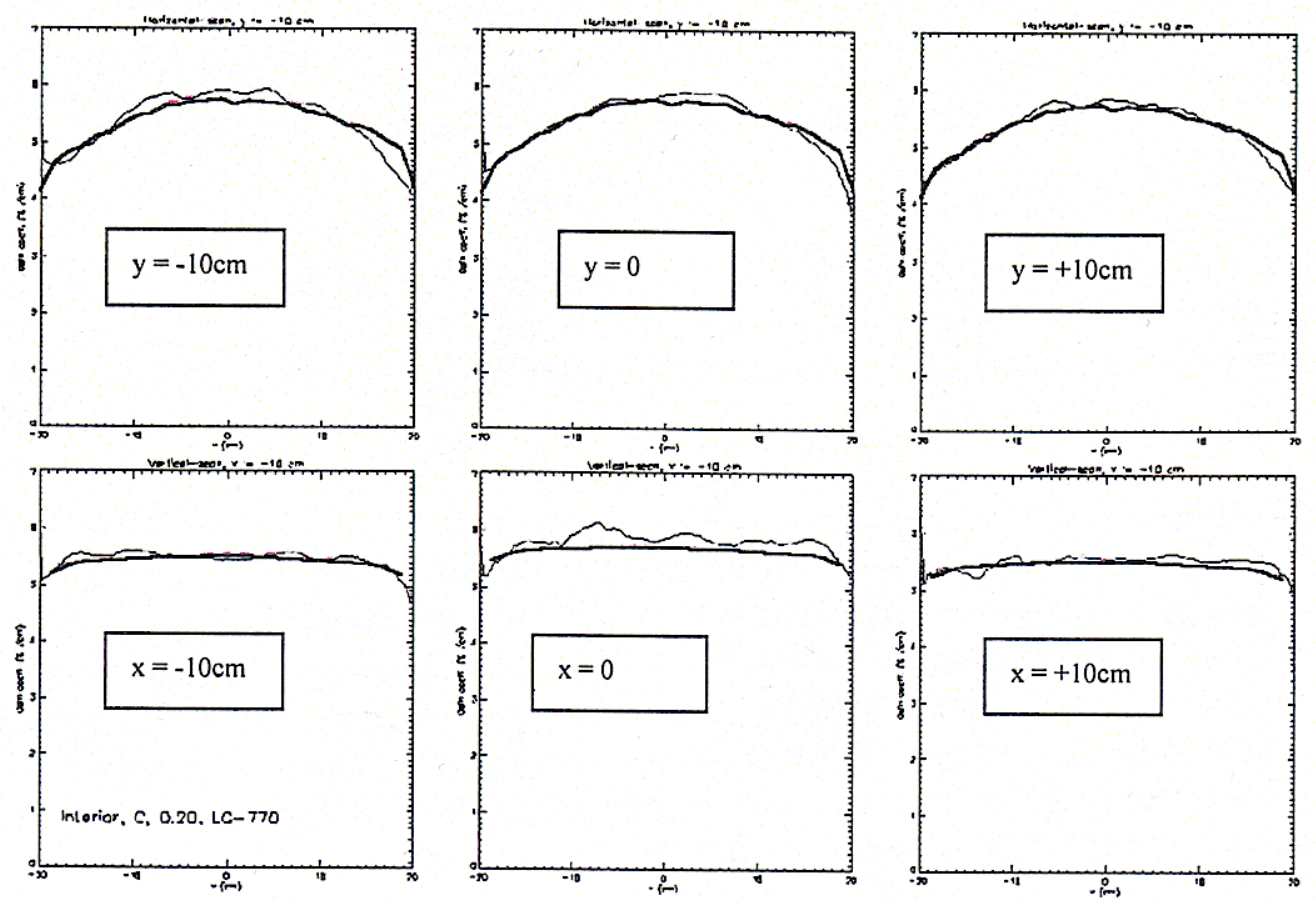

Figure 4.24. Line-out comparisons of the measured (light line) and predicted (bold line) gain coefficient profiles for the $\mathrm{C}$ aperture interior slab, for which full-aperture gain distribution are presented in Figures 4.23 and 4.24. Top row: horizontal line-outs at $y=-10,0$, and $+10 \mathrm{~cm}$; Bottom row: Vertical line-outs at $x=-10,0$ and $+10 \mathrm{~cm}$ 


\subsubsection{Comparison of the measurements and model predictions for the D (bottom) aperture}

\section{$X$ Configuration}

The next step is to see how well the model tracks the changes in the gain profile when we move from the $\mathrm{C}$ aperture near the center of the amplifier to the $\mathrm{D}$ aperture near the bottom reflector. The smoothed and shot-averaged experimental gain profile for the $\mathrm{D}$ aperture in the one-wide and two-wide versions of the $\mathrm{X}$ configuration at an explosion fraction of $20 \%$ are shown in Figures 4.7 and 4.8. The model prediction for this configuration is presented in Figure 4.25. Line-out comparisons of the measurement and prediction are plotted in Figure 4.26. The agreement between the model and the measurement is better than the experimental error (Figure 4.10) except in a few small regions, which appear to be the result of noise in the experimental signal. Notice that the model accurately predicts the slope of the vertical line-outs due to the presence of the reflector at the bottom of the aperture.

\section{Diamond Configuration}

The average experimental gain profile for this aperture and explosion fraction in the Diamond configuration is displayed in Figure 4.27. The model prediction is shown in Figure 4.28. Line-out comparisons of the two profiles are plotted in Figure 4.29. The model prediction is about $7 \%$ lower than the measurement along the vertical centerline of the aperture, but a portion of this discrepancy may come from experimental error which is larger $(\langle\Delta \propto\rangle=0.08 \% / \mathrm{cm})$ than the cases discussed above due to averaging over a smaller number of shots.

\subsubsection{Comparison of the measurements and model predictions for LHG-8 glass in the $A$ (top) aperture}

All of the measurements and calculations discussed so far have been for slabs in the lower 3 apertures (B, C, and D), which were filled with LG-770 laser glass. We now examine how well the model matches the experiment for the A aperture, which was populated with LHG-8 laser glass.

\section{$\mathrm{X}$ Configuration}

The smoothed and shot-averaged experimental gain profile for the A aperture in the two-wide $X$ configuration at an explosion fraction of $20 \%$ is shown in Figure 4.30. It is obvious from this figure that there is a large amount of noise in the experimental signal that could not be removed by the smoothing and averaging technique. This is because the two shots available for averaging in this configuration had similarly shaped, large-scale distortions. The model prediction for the gain profile is presented in Figure 4.31. Line-out comparisons of the measurement and prediction are plotted in Figure 4.32. In this case, the large distortions in the measured profile only allow us to say that the model and measurement for the average gain coefficient over the aperture agree to within the experimental error. 


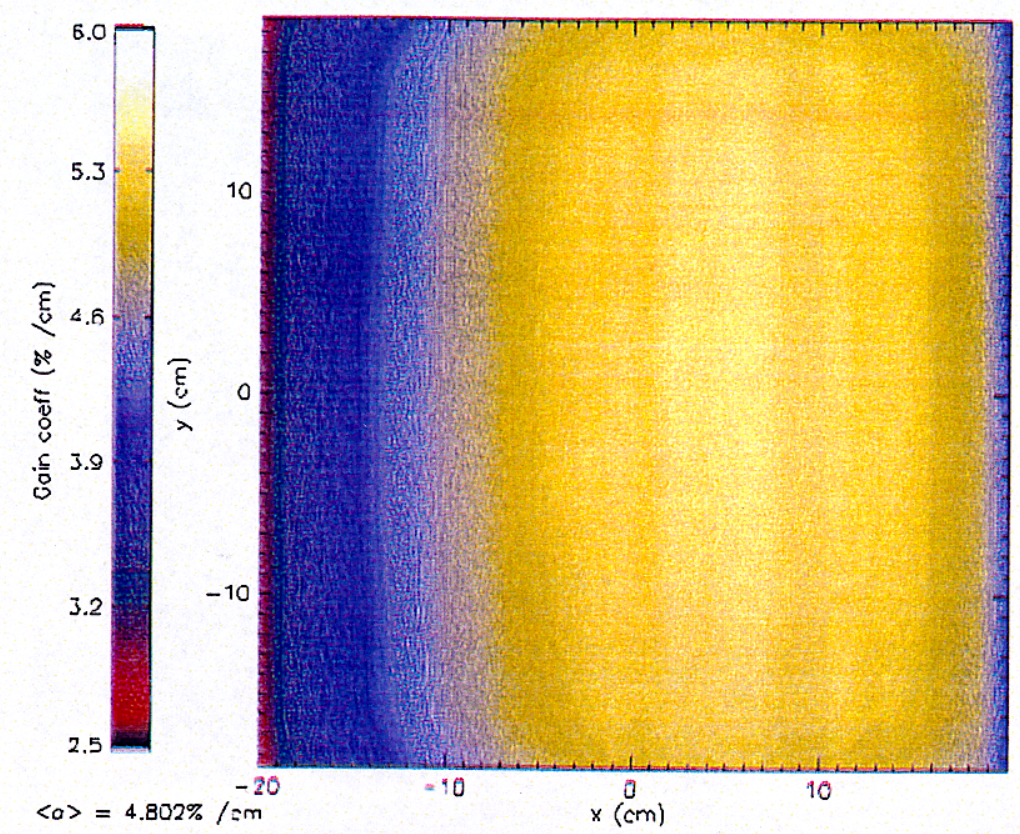

Figure 4.25. 3-D model prediction for the gain profile for the $D$ aperture in the $X$ configuration at a flashlamp explosion fraction of $20 \%$ (N.B.: $y$-axis limits in this plot are $\pm 18.5 \mathrm{~cm}$ ).
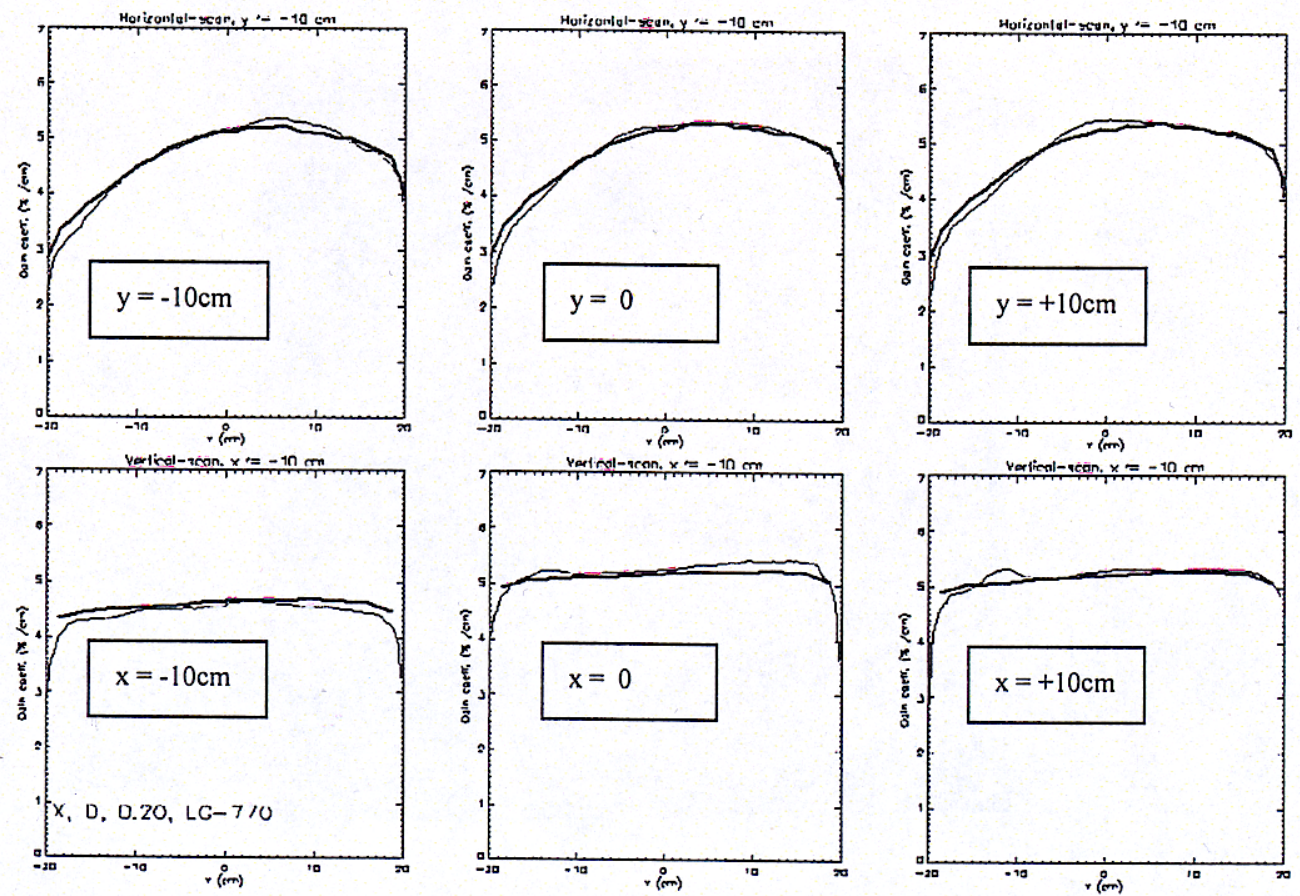

Figure 4.26. Line-out comparisons of the measured (light line) and predicted (bold line) gain coefficient profiles for the $D$ aperture in the $X$ configuration, for which full-aperture gain distribution are presented in Figures 4.8 and 4.26. Top row: horizontal line-outs at $y=-10,0$, and $+10 \mathrm{~cm}$; Bottom row: Vertical line-outs at $x=-10,0$ and $+10 \mathrm{~cm}$. 


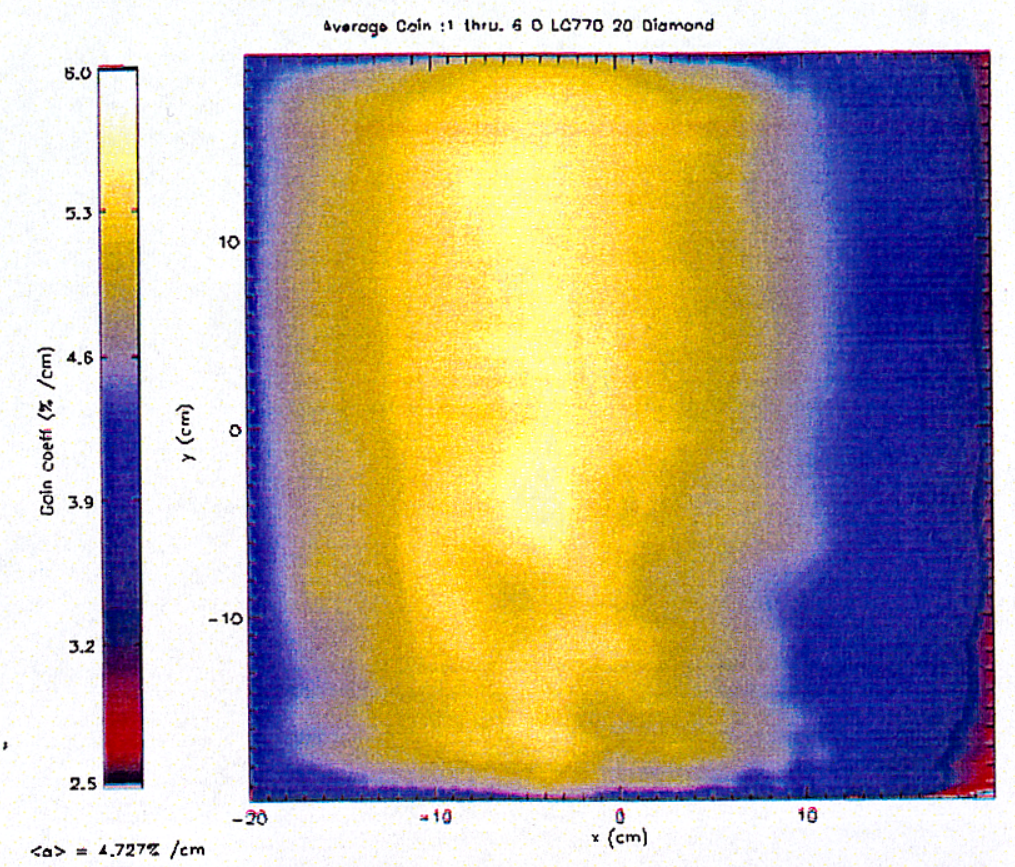

Figure 4.27. Smoothed and averaged gain profile for the $\mathrm{D}$ aperture in the two-wide Diamond configuration at a flashlamp explosion fraction of $20 \%$.

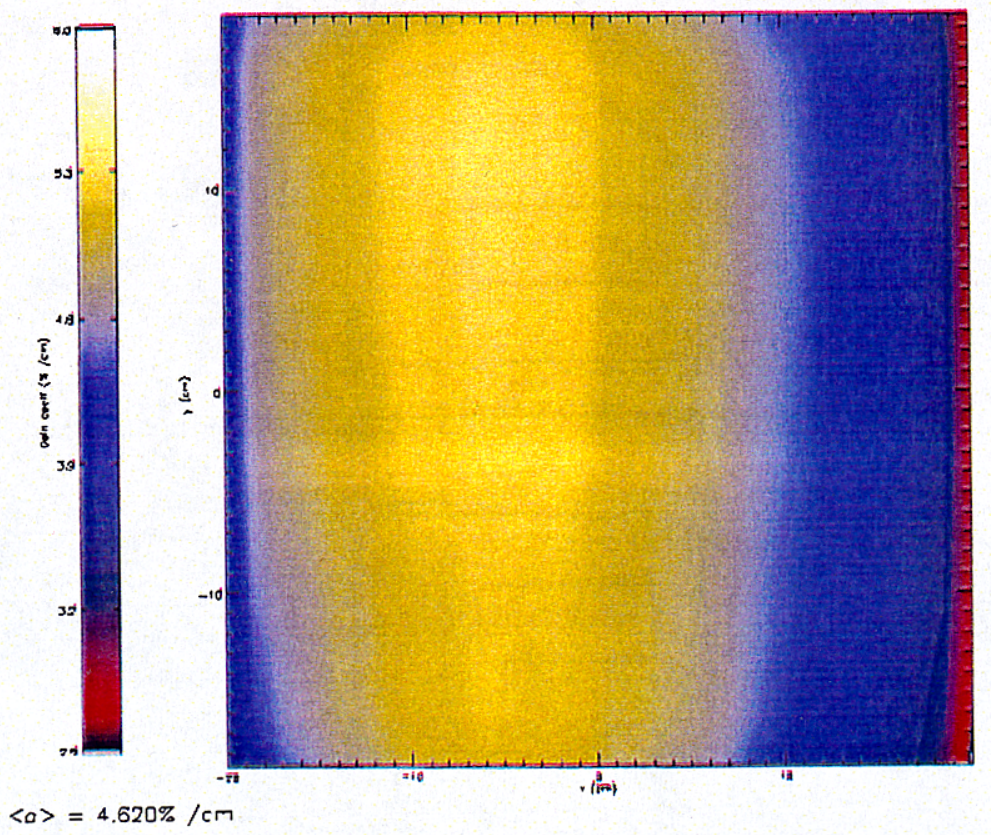

Figure 4.28. 3-D model prediction for the gain profile for the $D$ aperture in the Diamond configuration at a flashlamp explosion fraction of $20 \%$ (N.B.: $y$-axis limits in this plot are \pm $18.5 \mathrm{~cm})$. 

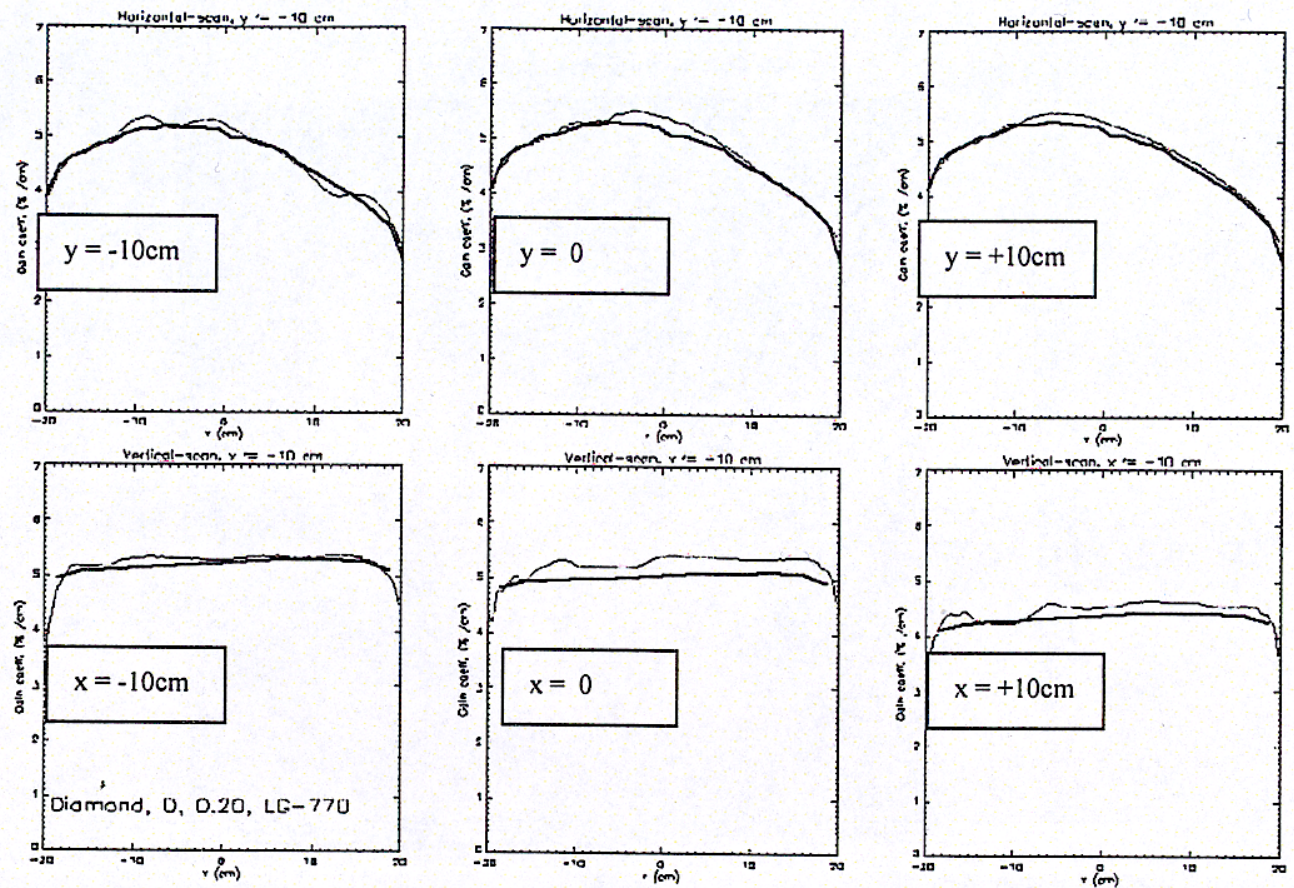

Figure 4.29. Line-out comparisons of the measured (light line) and predicted (bold line) gain coefficient profiles for the $\mathrm{D}$ aperture in the diamond configuration, for which full-aperture gain distribution are presented in Figures 4.8 and 4.26. Top row: horizontal line-outs at $y=-10,0$, and $+10 \mathrm{~cm}$; Bottom row: Vertical line-outs at $x=-10,0$ and $+10 \mathrm{~cm}$.

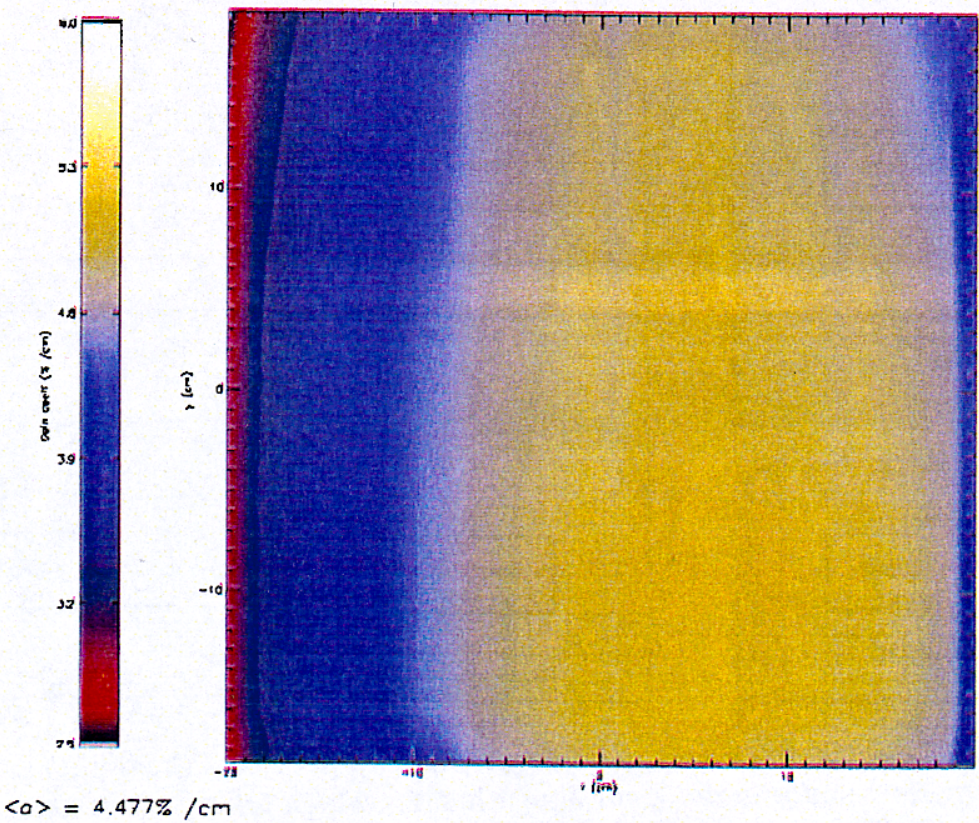

Figure 4.30. Smoothed and averaged gain profile for the $A$ aperture in the two-wide $X$ configuration at a flashlamp explosion fraction of $20 \%$. 


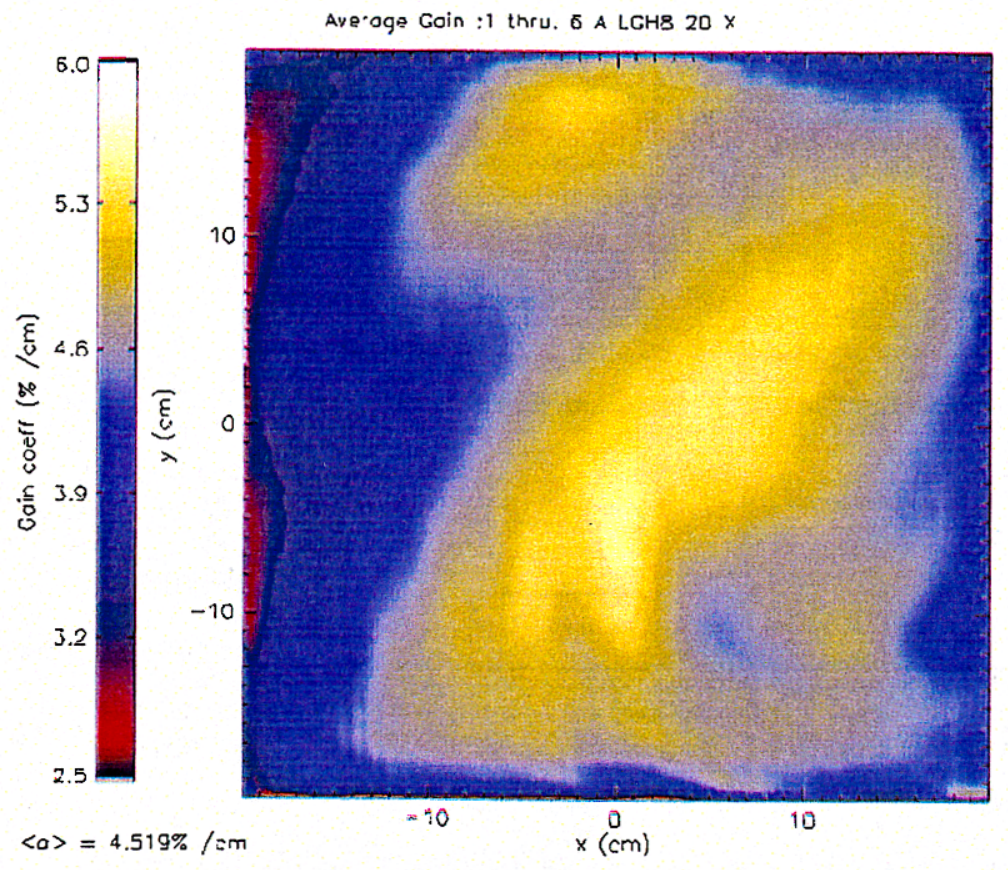

Figure 4.31. 3-D model prediction for the gain profile for the $\mathrm{A}$ aperture in the two-wide $\mathrm{X}$ configuration at a flashlamp explosion fraction of $20 \%$ (N.B.: $y$-axis limits in this plot are $\pm 18.5 \mathrm{~cm}$ ).
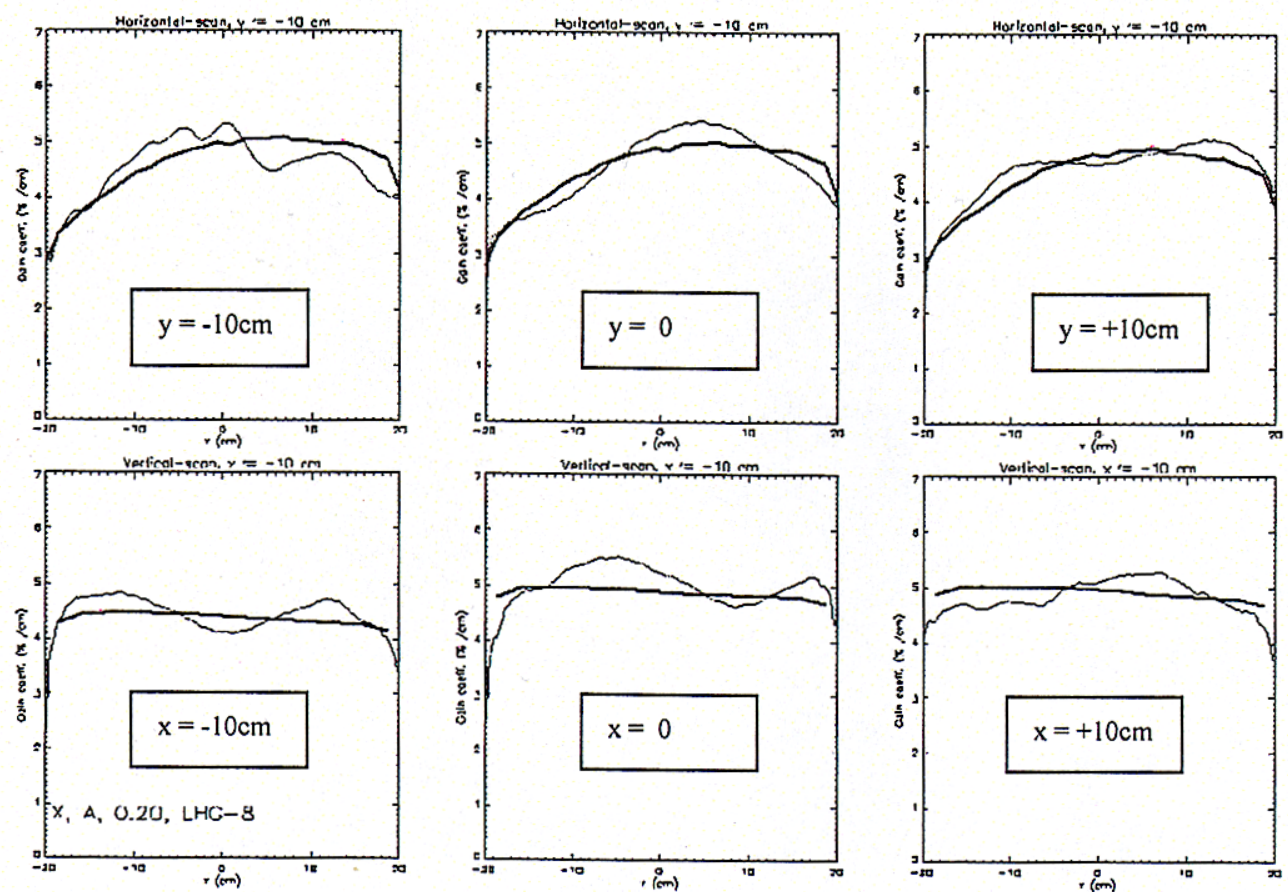

Figure 4.32. Line-out comparisons of the measured (light line) and predicted (bold line) gain coefficient profiles for the $A$ aperture in the $X$ configuration, for which full-aperture gain distribution are presented in Figures 4.31 and 4.32. Top row: horizontal line-outs at $y=-10,0$, and $+10 \mathrm{~cm}$; Bottom row: Vertical line-outs at $x=-10,0$ and $+10 \mathrm{~cm}$. 


\section{Configuration}

The situation is better for the gain profiles for the $A$ aperture in the Diamond configuration at an explosion fraction of $20 \%$. The average experimental gain profile is displayed in Figure 4.33. The model prediction for the gain is presented in Figure 4.34. Line-out comparisons of two profiles are plotted in Figure 4.35. There is good agreement between the model over most of the aperture, and the regions in which the two curves differ significantly can be identified as those in which the error in the average of the experimental gain profiles is large.

\subsubsection{Comparison of the measurements and model predictions for different explosion fractions}

The last thing that we have to check is that the model correctly predicts the gain profile at different explosion fractions. The smoothed experimental gain profile for the $D$ aperture in the two-wide $\mathrm{X}$ configuration at an explosion fraction of $15 \%$ is presented in Figure 4.36 (there is no averaging because there were no configurations for which there is more than one gain data file at an explosion fraction of $15 \%$ ). The model prediction for the gain profile is displayed in Figure 4.37. Line-out comparisons between the two profiles are plotted in Figure 4.38. The model appears to underestimate the gain in this case by about $8 \%$, but we have no data to assess the statistical relevance of this discrepancy.

The smoothed and averaged gain profile for the $\mathrm{D}$ aperture in the two-wide $\mathrm{X}$ configuration at an explosion fraction of $23 \%$ is shown in Figure 4.39. The model prediction for the gain profile for this configuration is presented in Figure 4.40. Line-out comparisons between the measurement and the model predictions are shown in Figure 4.41. While there is good agreement between the model and the measurement over most of the aperture, the model appears to underestimate the roll-off in the gain on the weak side by about $8 \%$. This may be an indication that we are slightly underestimating the ASE decay rate, but part of the disagreement may also come from the fact that the error in the measured profile is highest in this region of the aperture.

\subsubsection{Conclusions}

The comparisons illustrated above demonstrate that the 3-D model can be used to predict the gain distributions over the range of amplifier parameters relevant to NIF performance. From this analysis, we estimate that 3-D model predictions have errors of $\pm 5 \%$ for local values of the gain coefficient at specific locations in the aperture and errors of $\pm 2 \%$ for the aperture-averaged gain coefficient. 


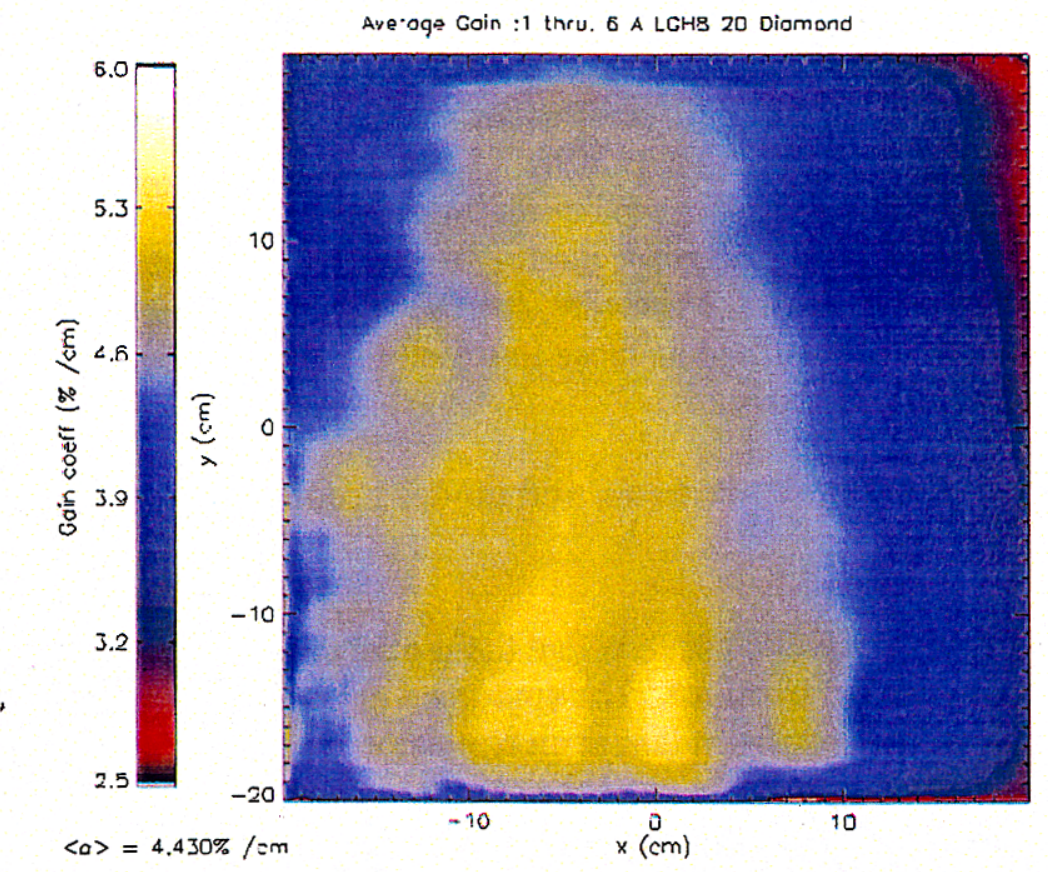

Figure 4.33. Smoothed and averaged gain profile for the $A$ aperture in the two-wide diamond configuration at a flashlamp explosion fraction of $20 \%$.

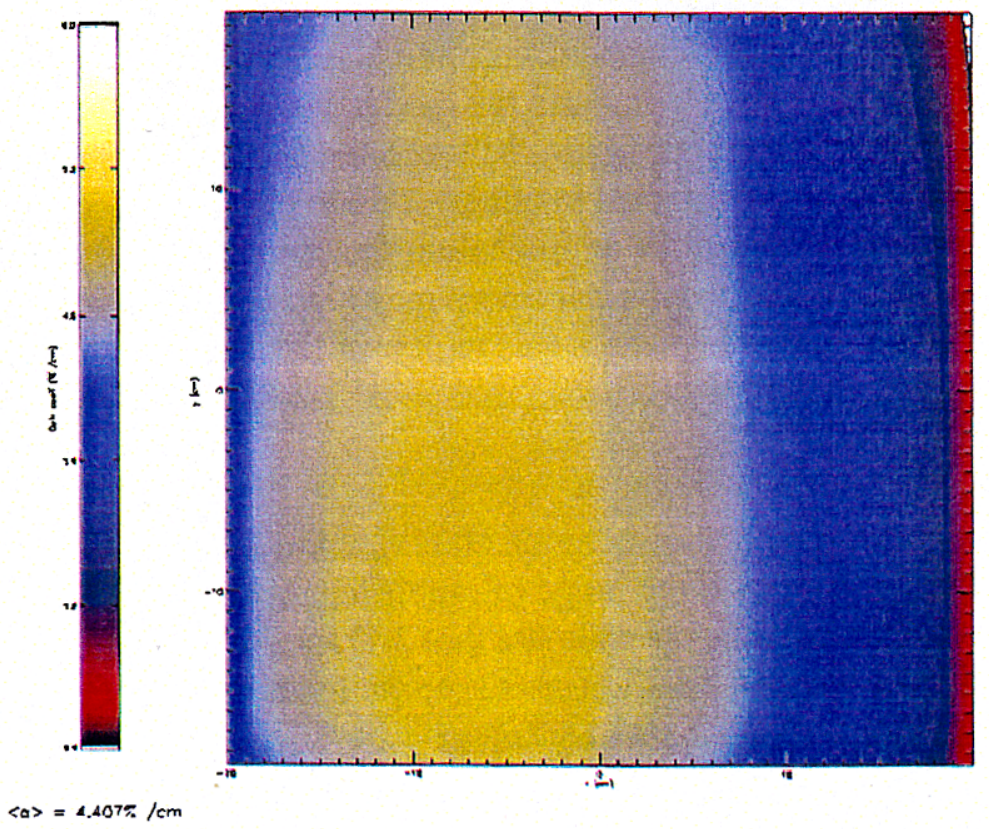

Figure 4.34. 3-D model prediction for the gain profile for the $A$ aperture in the two-wide diamond configuration at a flashlamp explosion fraction of $20 \%$ (N.B.: $y$-axis limits in this plot are $\pm 18.5 \mathrm{~cm}$ ). 

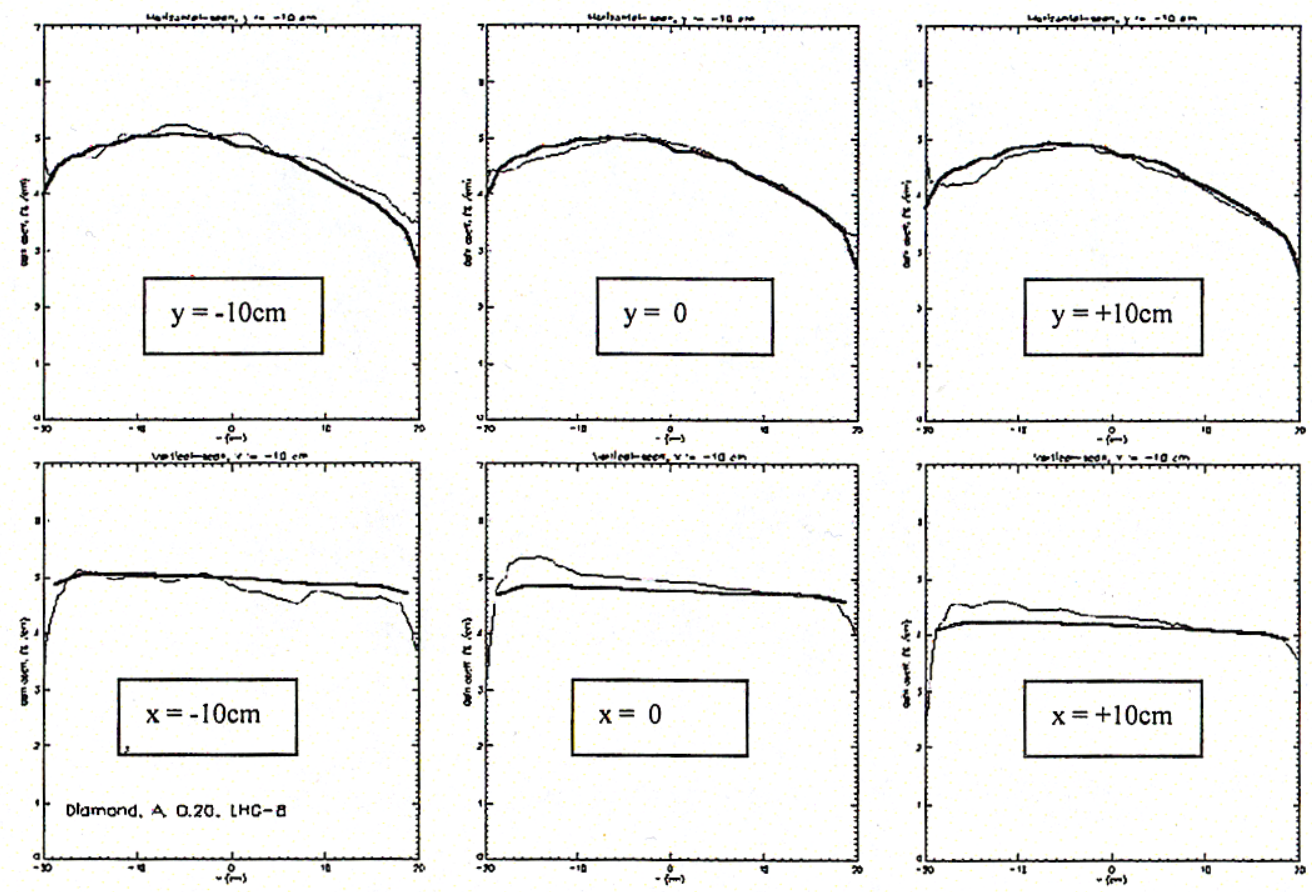

Figure 4.35. Line-out comparisons of the measured (light line) and predicted (bold line) gain coefficient profiles for the $A$ aperture in the diamond configuration, for which full-aperture gain distribution are presented ofiles in Figures 4.34 and 4.35. Top row: horizontal line-outs at $y=-10$, 0 , and $+10 \mathrm{~cm}$; Bottom row: Vertical line-outs at $x=-10,0$ and $+10 \mathrm{~cm}$.

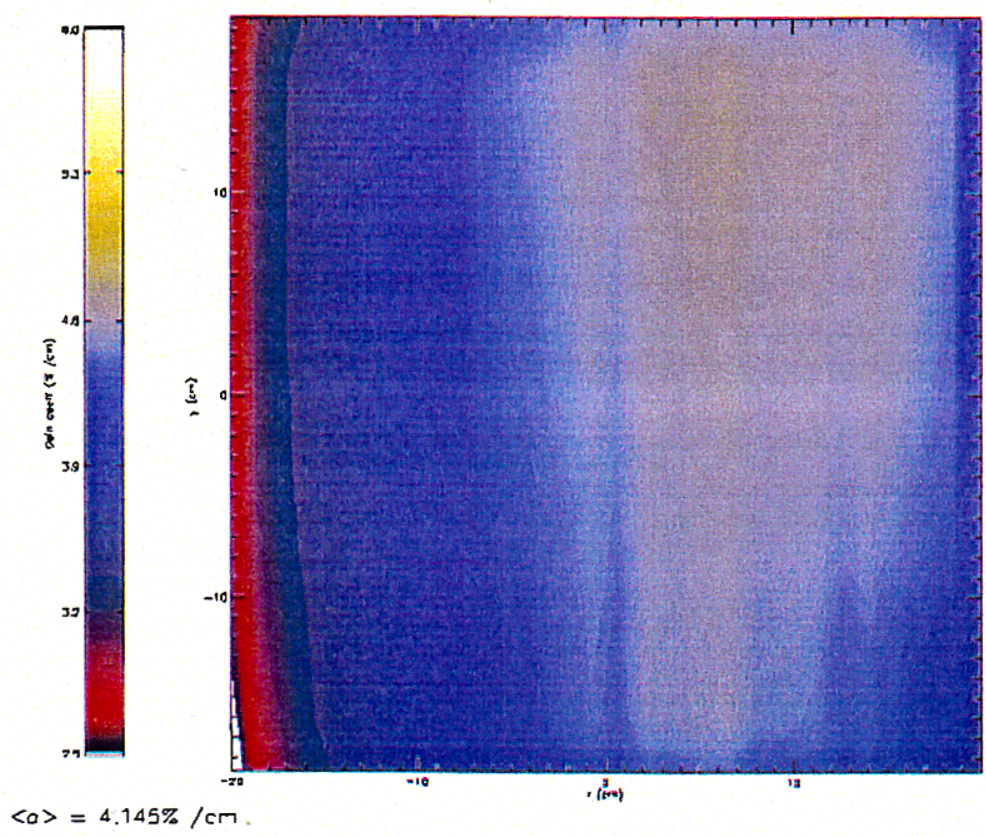

Figure 4.36. Smoothed gain profile for the $D$ aperture in the two-wide $X$ configuration at a flashlamp explosion fraction of $15 \%$. 


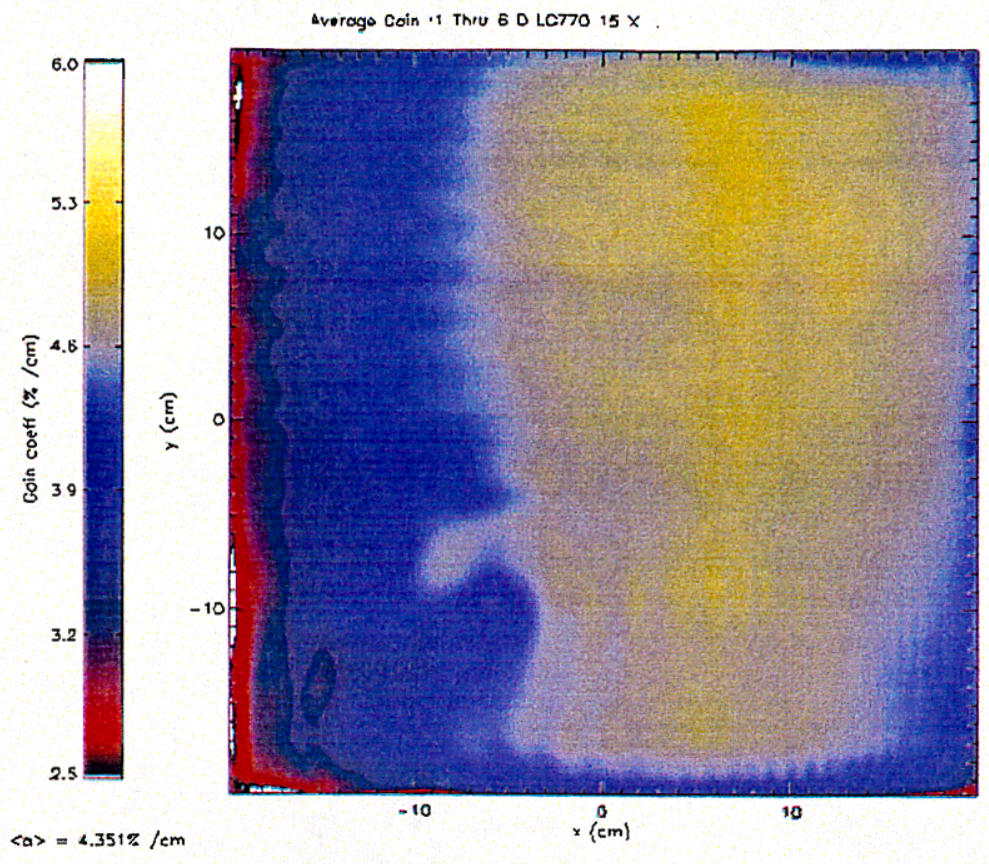

Figure 4.37. 3-D model prediction for the gain profile for the $D$ aperture in the two-wide $X$ configuration at a flashlamp explosion fraction of $15 \%$ (N.B.: $y$-axis limits in this plot are $\pm 18.5 \mathrm{~cm}$ ).
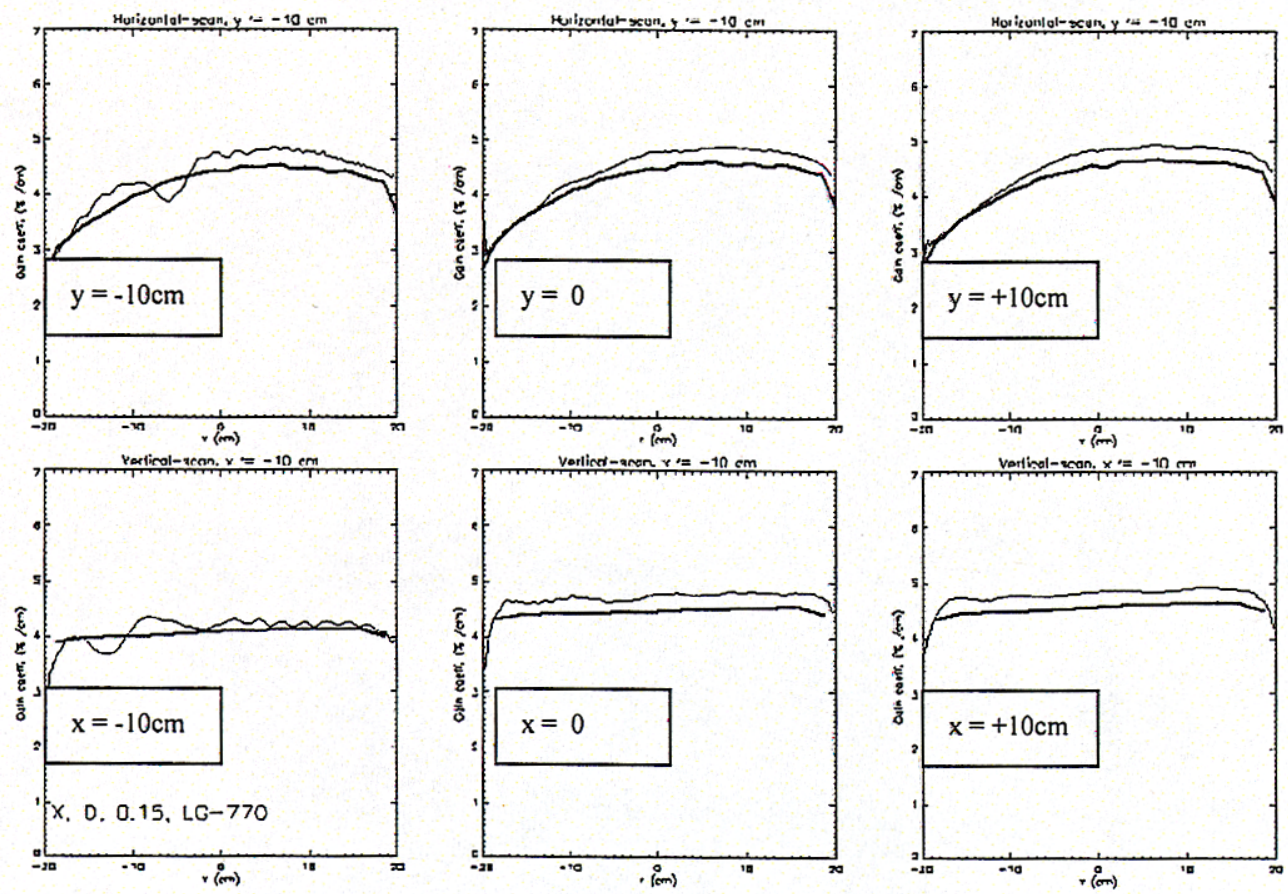

Figure 4.38. Line-out comparisons of the measured (light line) and predicted (bold line) gain coefficient profiles for the $D$ aperture in the $X$ configuration at a flashlamp explosion fraction of 0.15 , for which full-aperture gain distribution are presented in Figures 4.37 and 4.38. Top row: horizontal line-outs at $y=-10,0$, and $+10 \mathrm{~cm}$; Bottom row: Vertical line-outs at $x=-10,0$ and $+10 \mathrm{~cm}$. 


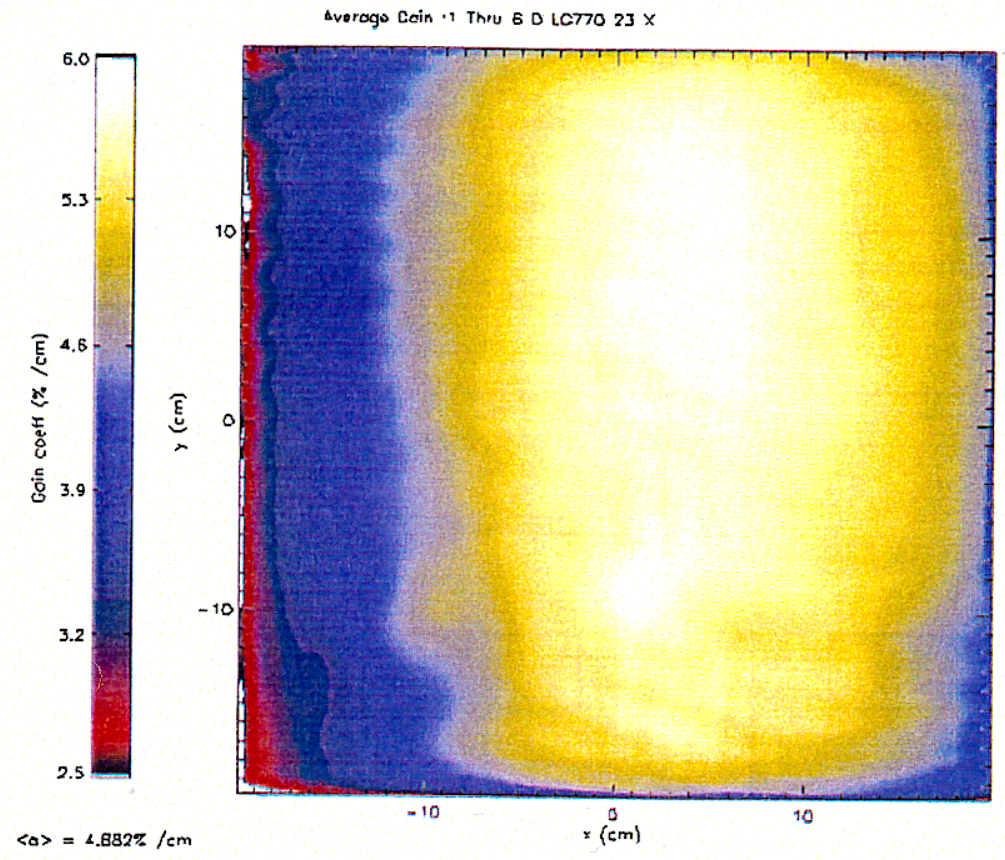

Figure 4.39. Smoothed and averaged gain profile for the $\mathrm{D}$ aperture in the two-wide $\mathrm{X}$ configuration at a flashlamp explosion fraction of $23 \%$.

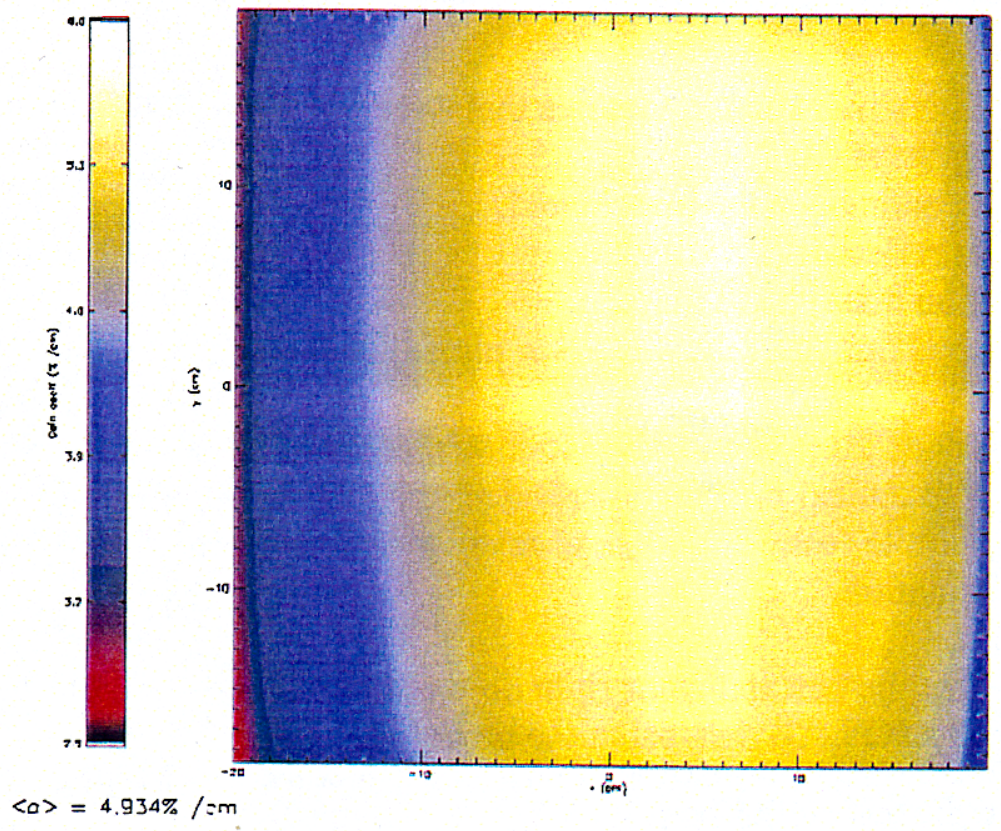

Figure 4.40. 3-D model prediction for the gain profile for the $D$ aperture in the two-wide $X$ configuration at a flashlamp explosion fraction of $23 \%$ (N.B.: $y$-axis limits in this plot are $\pm 18.5 \mathrm{~cm}$ ). 

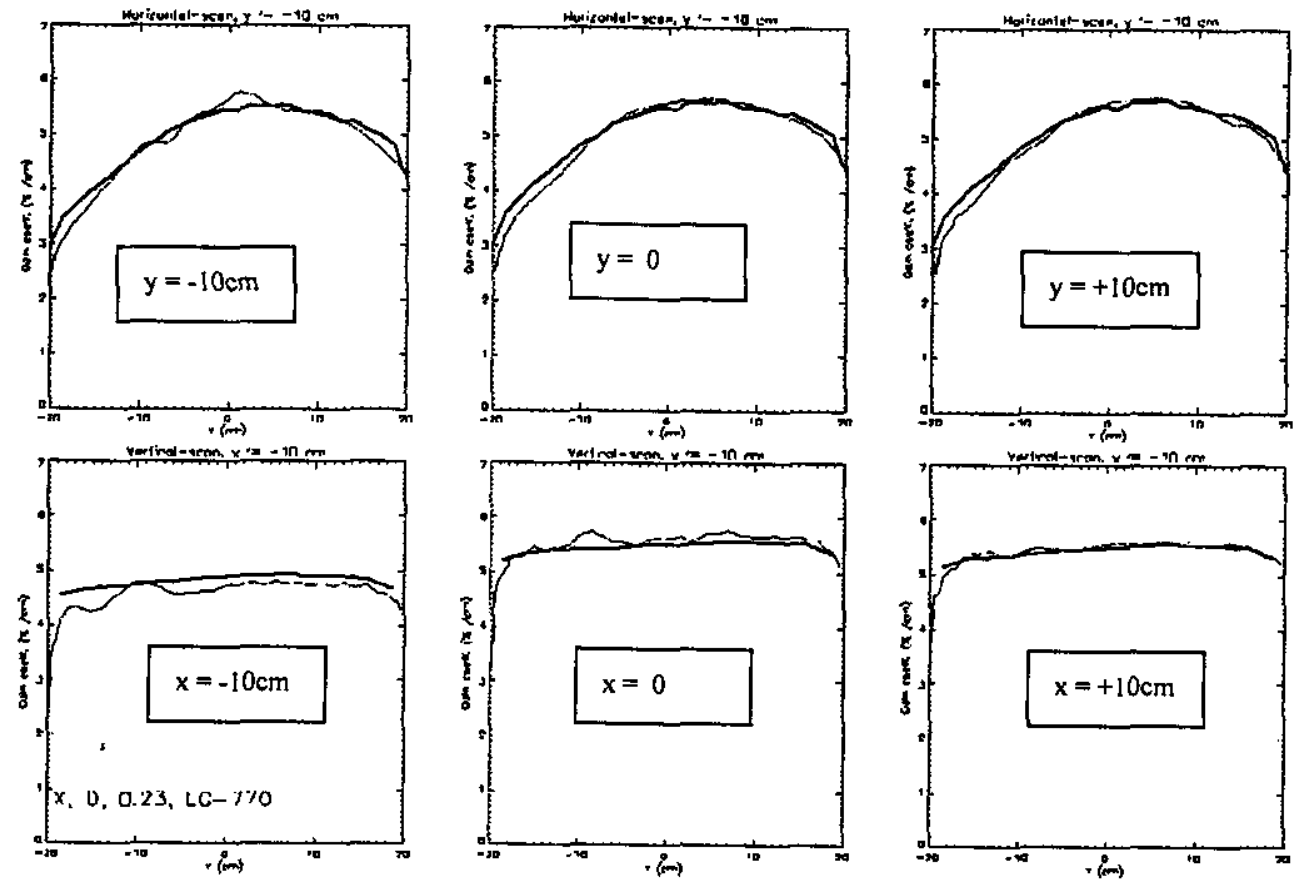

Figure 4.41. Line-out comparisons of the measured (light line) and predicted (bold line) gaincoefficient profiles for the $D$ aperture in the $X$ configuration at a flashlamp explosion fraction of 0.23 , for which full-aperture gain distribution are presented in Figures 4.40 and 4.41. Top row: horizontal line-outs at $y=-10,0$, and $+10 \mathrm{~cm}$; Bottom row: Vertical line-outs at $x=-10,0$ and $+10 \mathrm{~cm}$.

\subsection{Gain Predictions}

The NIF amplifiers will contain features that were not present in our earlier amplifiers. Among these are:

- Two-layer AR coatings on the blast shield.

- Protected silver reflectors.

- Lower-absorption blast shield glass.

- Triangular-shaped (an possibly non-silver plated) slab masks.

Our 3-D amplifier code must be modified to model some of these changes. For others, we do not currently have optical information needed to maintain model accuracy. For these reasons, we are not quite ready to do a new calculation that could accurately be labeled a "NIF amplifier simulation."

Instead, we think that the best indicator right now of the NIF performance is an extrapolation of the AMPLAB data using the model with improved silver reflectors $(96 \%$ reflectivity at $670 \mathrm{~nm}$, as opposed to $91 \%$ in AMPLAB) that are more representative of what we will have in the NIF amplifiers.

We used the 3-D code with the increased reflectivity to calculate new gain profiles for the slabs in the $\mathrm{C}$ aperture at an explosion fraction of $20 \%$. We extrapolated the calculated profiles out to the vertical edges of the aperture at $\pm 20 \mathrm{~cm}$ by assuming that there is a 
$12.5 \%$ roll-off over the last $1.33 \mathrm{~cm}$. This value for the gain roll-off was arrived at by fitting the model to the profiles measured in AMPLAB. The quality of the fit is illustrated in the agreement between the vertical line-outs in the bottom row of Figure 4.42.

The extrapolated AMPLAB gain profiles with $96 \%(670 \mathrm{~nm})$ reflectors for the $\mathrm{X}$, diamond, and interior slab positions in the $\mathrm{C}$ aperture at an explosion fraction of $20 \%$ are presented in Figures 4.43 through 4.45. As one would expect, the improved reflectivity increases the average gain coefficient. Using these gain profiles, the beam-averaged gain coefficient for a NIF chain composed of equal numbers of LG-770 and LHG-8 glass slabs is $5.23 \% \mathrm{~cm}^{-1}$. The increased reflectivity also improves the gain uniformity, as the beam-averaged peak-to-average ratio decreases to 1.07 . We attribute this result to the fact that the shaped reflectors are specifically designed to direct light to the edges of the slab to reduce the roll-off in the gain caused by ASE. The fact that the peak-to-average ratio decreases with increased reflectivity simply shows us that the reflectors are indeed serving to improve the gain uniformity.

Chain-averaged gain distributions are presented in Section 7, Implications for NIF Performance.
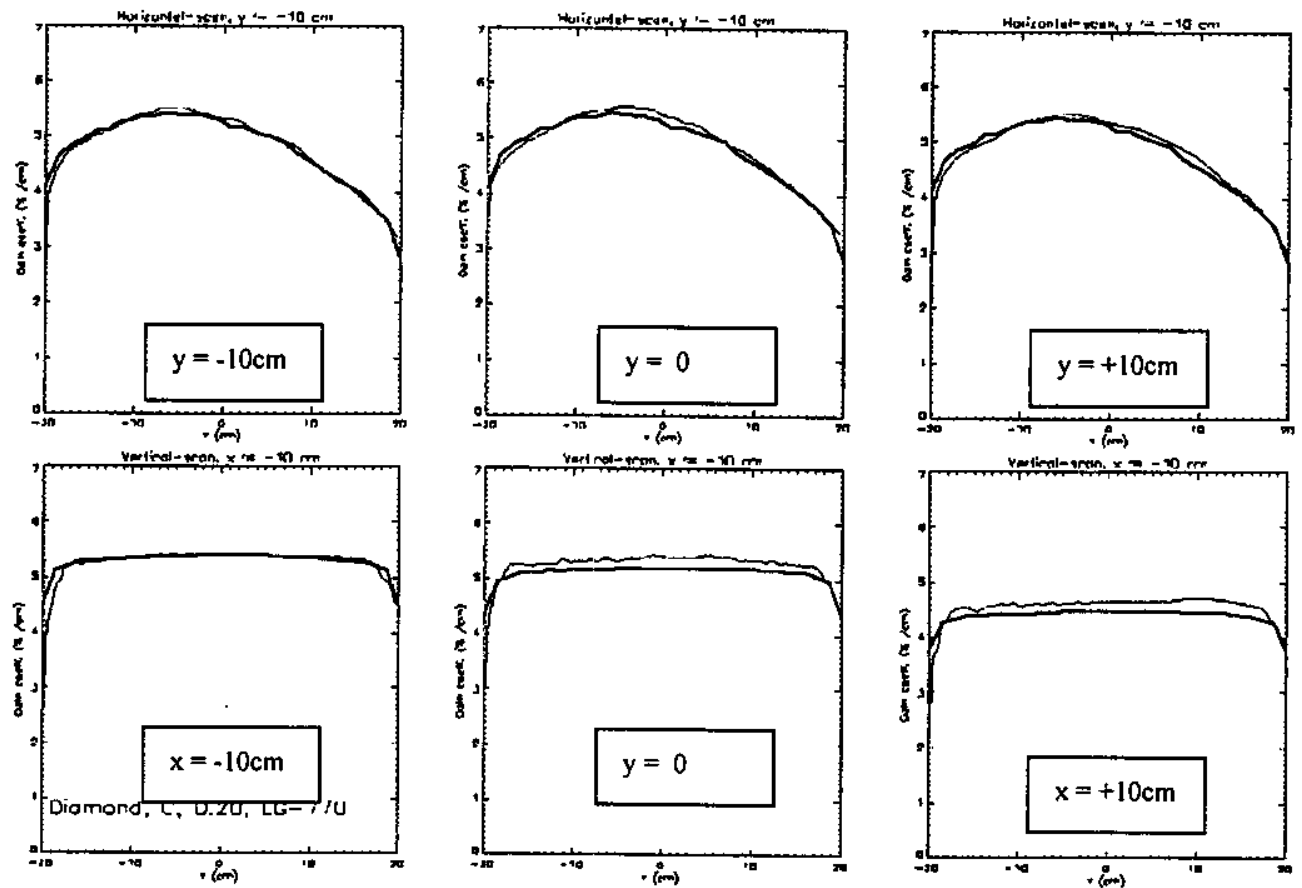

Figure 4.42. Line-out comparisons of the measured (light line) and predicted (with extrapolation out to $\pm 20 \mathrm{~cm}$ ] (bold line) gain coefficient profiles in AMPLAB for the C aperture, Diamond configuration, at an explosion fraction of $20 \%$. Top row: horizontal line-outs at $y=-10,0$, and +10 $\mathrm{cm}$; Bottom row: Vertical line-outs at $x=-10,0$ and $+10 \mathrm{~cm}$. 


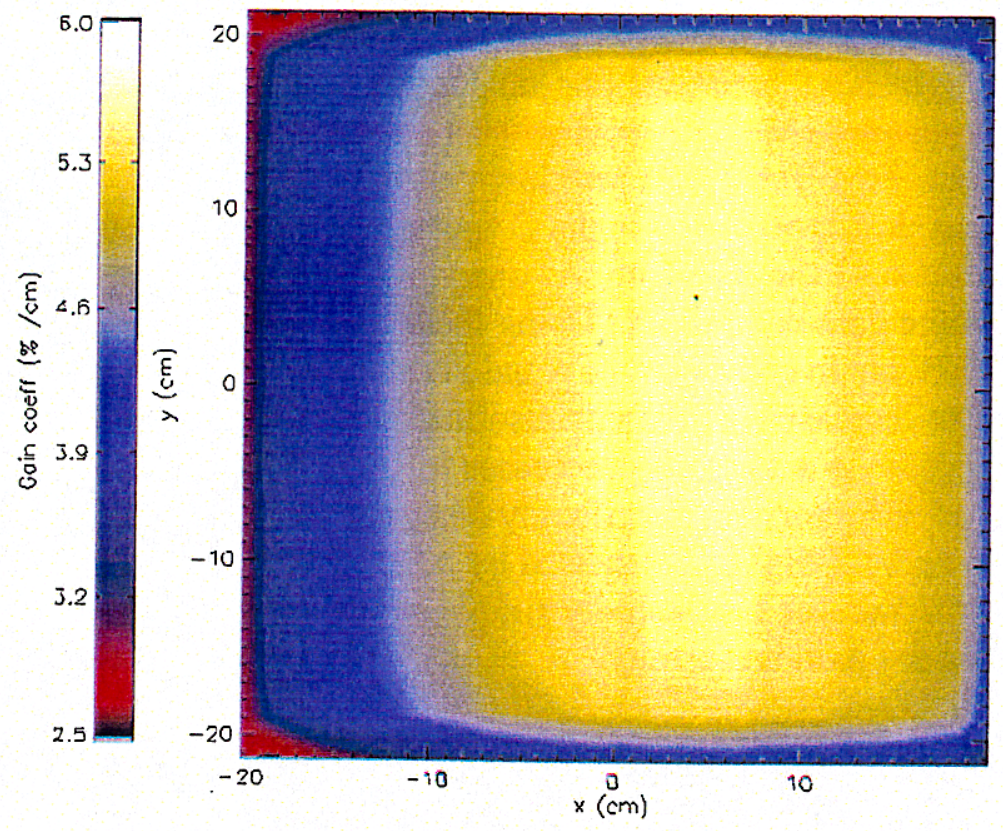

Figure 4.43. 3-D model prediction for the gain profile for the $\mathrm{C}$ aperture in an $\mathrm{X}$-type end slab at a flashlamp explosion fraction of $20 \%$ with $96 \%$ (@650 $\mathrm{nm}$ ) reflectors (N.B.: the data beyond $\pm 20 \mathrm{~cm}$ in the vertical direction is an artifact of the plotting process).

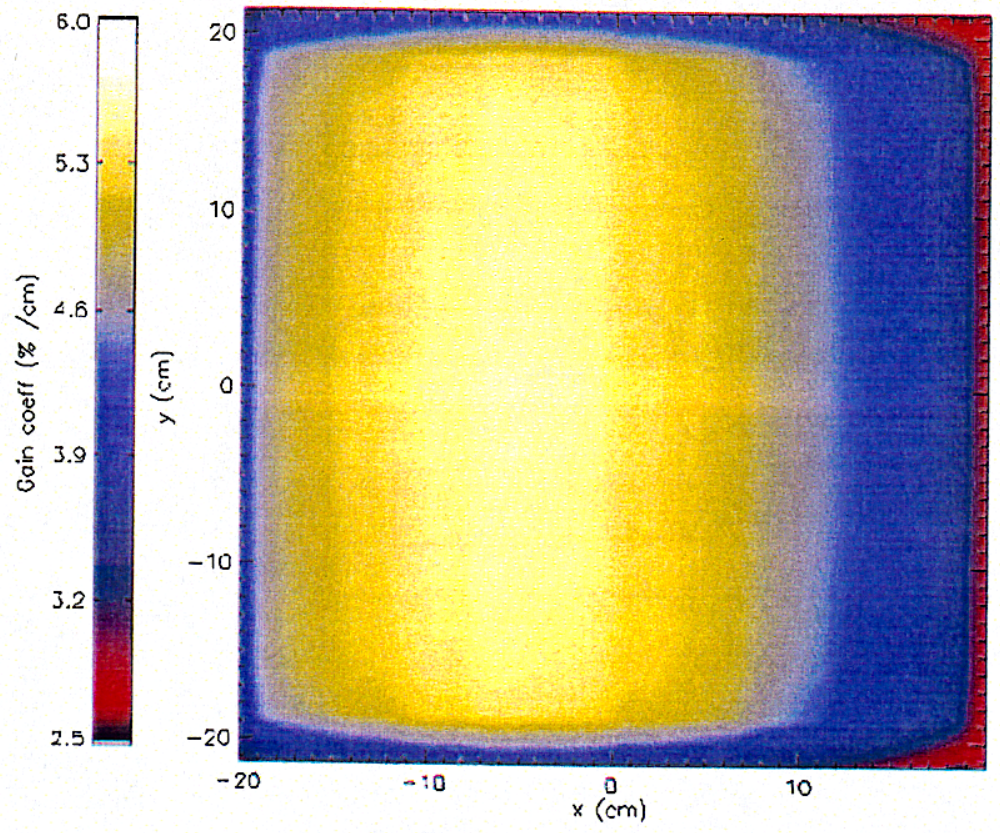

Figure 4.44. 3-D model prediction for the gain profile for the $\mathrm{C}$ aperture in an Diamond-type end slab at a flashlamp explosion fraction of $20 \%$ with $96 \%$ (650 $\mathrm{nm}$ ) reflectors (N.B.: the data beyond $\pm 20 \mathrm{~cm}$ in the vertical direction is an artifact of the plotting process). 


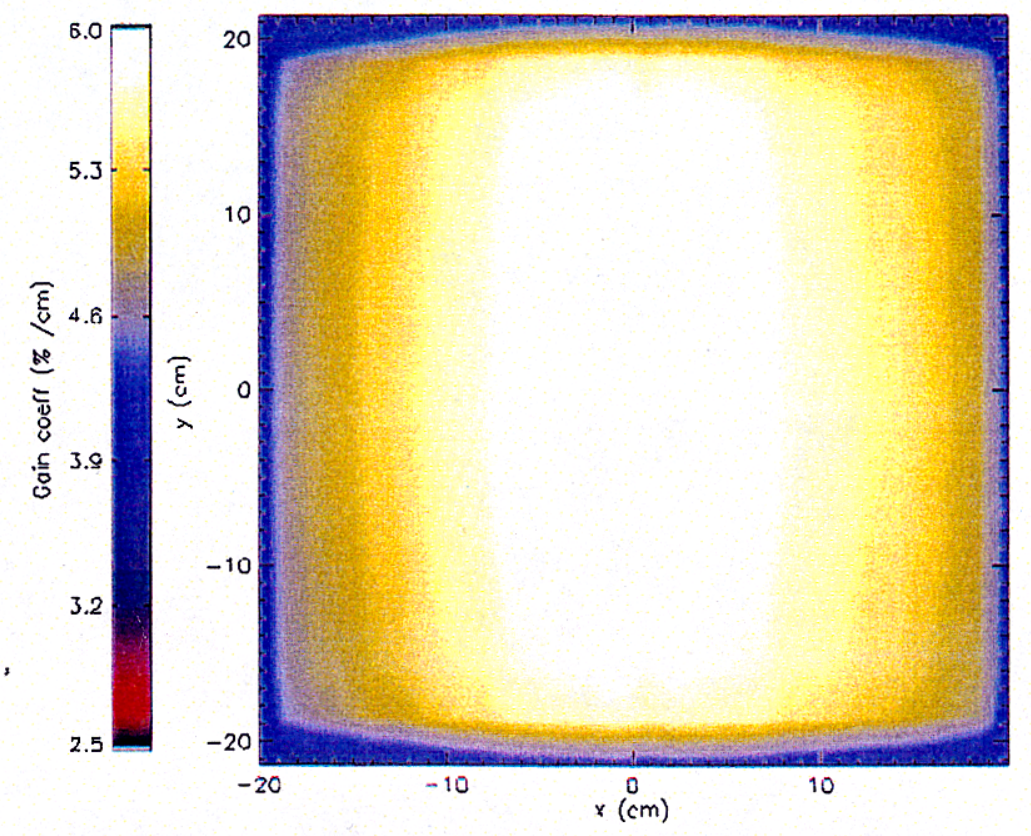

Figure 4.45. 3-D model prediction for the gain profile for the $C$ aperture, interior slab at a flashlamp explosion fraction of $20 \%$ with $96 \%$ (@650 $\mathrm{nm}$ ) reflectors (N.B.: the data beyond $\pm 20 \mathrm{~cm}$ in the vertical direction is an artifact of the plotting process). 
7 


\section{Prompt Pump-Induced Wavefront Measurements and Modeling}

This section contains prompt pump-induced wavefront distortion studies, which includes measurements made in AMPLAB, modeling, comparison of model predictions with measurements, and predictions for the NIF laser.

\subsection{Mechanism}

The NIF laser design uses $4 \times 2$ multisegment amplifiers in which four-slab-high columns of laser slabs, oriented at the Brewster angle with respect to the laser beam, are pumped by central flashlamp arrays on one side and by side flashlamp arrays on the other side. The flashlamp pump rate is nonuniform, with the regions of the slab that are nearest the flashlamps being pumped more strongly than the regions that are farther away. Because a fraction of the flashlamp pump light absorbed by the slab is converted into thermal energy, the resulting thermal expansion causes the laser slab to warp into a characteristic "S" shape [5.1] (see Figure 5.1). This slab warping occurs on a time scale comparable to the duration of the flashlamp pump pulse and causes distortion of the laser-beam wavefront. Additional wavefront distortion is produced by refractive index variations caused by pump-induced temperature variations and stress. Because the slab is oriented at the Brewster's angle and most of the slab warping occurs in the horizontal plane, the phase gradients are larger in the horizontal direction than in the vertical direction.

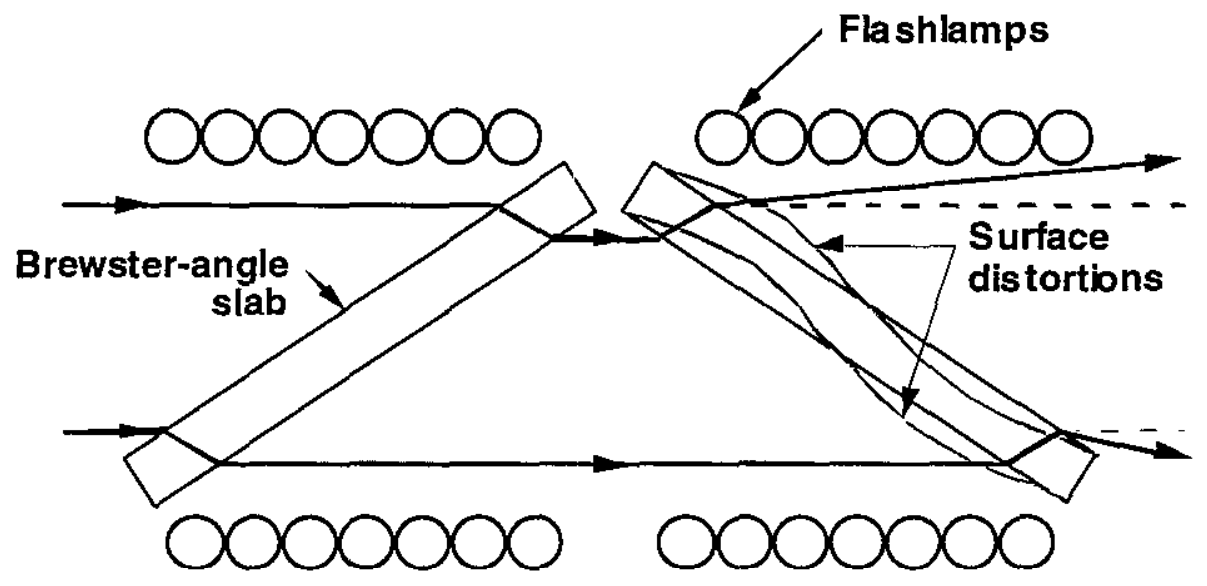

Figure 5.1. Plan view of multisegment amplifier showing geometry of Brewster angle laser slabs. Surface distortions (greatly exaggerated) caused by uneven pumping.

\subsection{Data analysis method}

As described in Section 3.2, prompt pump-induced wavefront distortion produced by the AMPLAB prototype amplifier was determined by subtracting the phase distribution 
measured at the time of peak gain with one camera from the phase distribution measured $\sim 75 \mathrm{~ms}$ before the flashlamps were fired with another camera. Phase distributions were inferred from interferograms produced with a pulsed, single-frequency $\mathrm{Nd}: Y \mathrm{YLF}$ probe laser and a Twyman-Green interferometer. The probe laser beam was double-passed through the prototype amplifier, while the reference beam was generated using a polarization-preserving, single-mode optical fiber. Two separate CCD cameras were used to record interferograms produced on successive pulses of the probe laser, which operated at a repetition rate of $13 \mathrm{~Hz}$.

Unlike traditional interferograms, which have only a few fringes across the aperture, the interferograms produced in AMPLAB typically had approximately 80 fringes, which were generated by tilting the reference beam relative to the probe beam. Thus, the phase information resided in a high-frequency carrier signal that could be readily separated from the typical low-frequency amplitude variations using a Fourier transform method [5.2, 5.3]. The algorithm used to analyze the AMPLAB interferograms performed a discrete Fourier transform on the raw CCD camera images, extracted the phase information from a predetermined region of the Fourier spectrum, relocated the extracted signal to the origin, and performed an inverse Fourier transform to generate the phase distribution.

The algorithm function returns values between $-\pi$ and $\pi$, producing $2 \pi$ discontinuities in phase that are nonphysical. These discontinuities are unwrapped into continuous phase space using a routine written by Henesian [5.4].

Recall that the prompt pump-induced wavefront distortion was determined from the difference between phase distributions inferred from the interferograms recorded on two separate cameras. One camera recorded the interferogram at peak gain, while the other recorded the interferogram $\sim 75 \mathrm{~ms}(1 / 13 \mathrm{~Hz})$ before the flashlamps were fired. Since both cameras were affected by the 8-10 waves (mainly curvature) of static distortion in the LADS optics, the measured prompt pump-induced wavefront distortion was extremely sensitive to registration errors between the two camera images. With assistance from Paul Renard, we developed an algorithm that accurately located and aligned the two images and corrected for magnification differences. Rotation errors were sufficiently small that no correction for them was required. Our technique did not require any auxiliary images, such as cross-hair images or zero-delay corrections. The registration error of 1-2 pixels that was achieved corresponded to a wavefront error of $\sim 0.01$ waves $(\mathrm{P}-\mathrm{V})$.

We also investigated various techniques that minimized the amount of edge distortion introduced by the calculation. We tried various windowing techniques and found that most reasonable windows produced about the same results. However, the AMPLAB data was analyzed using a Von Hann $\left(a \cos ^{\alpha} x\right)$ window [5.3]. 


\subsection{AMPLAB measurement results}

The prompt pump-induced wavefront measurements made in AMPLAB on diamond, $\mathrm{X}$, and 3-slab-long amplifier configurations form a self-consistent and relatively complete set from which we were able to determine the prompt pump-induced wavefront distortion of the interior slab. All measurements in this set were taken in the third aperture from the top of the amplifier (i.e., the " $\mathrm{C}$ " aperture), which was outfitted with LG-770 laser glass. The flashlamps were fired at an explosion fraction of 0.20 , the expected normal operating point for the NIF flashlamps. Three shots were taken for each condition.

Figures 5.2 and 5.3 show 3D representations of the phase distortion measured on the diamond and $\mathrm{X}$ configurations, respectively. The results have been averaged over three shots and normalized on a per-slab-pass basis. Fitting the data to a sixth-order polynomial removed high-frequency structure believed to be noise from optical defects, without removing important phase information.

Figure 5.4 shows the phase distortion for the interior slab, $\phi_{\mathrm{i}}$, which was inferred from the data using the formula

$$
\phi_{i}=\frac{1}{2} \phi_{3}-\frac{1}{4}\left(\phi_{d}+\phi_{x}\right),
$$

where $\phi_{d}, \phi_{x}$, and $\phi_{3}$ are the prompt pump-induced wavefront distortion (phase) measured on the diamond, $\mathrm{X}$, and 3-slab-long configurations, respectively.
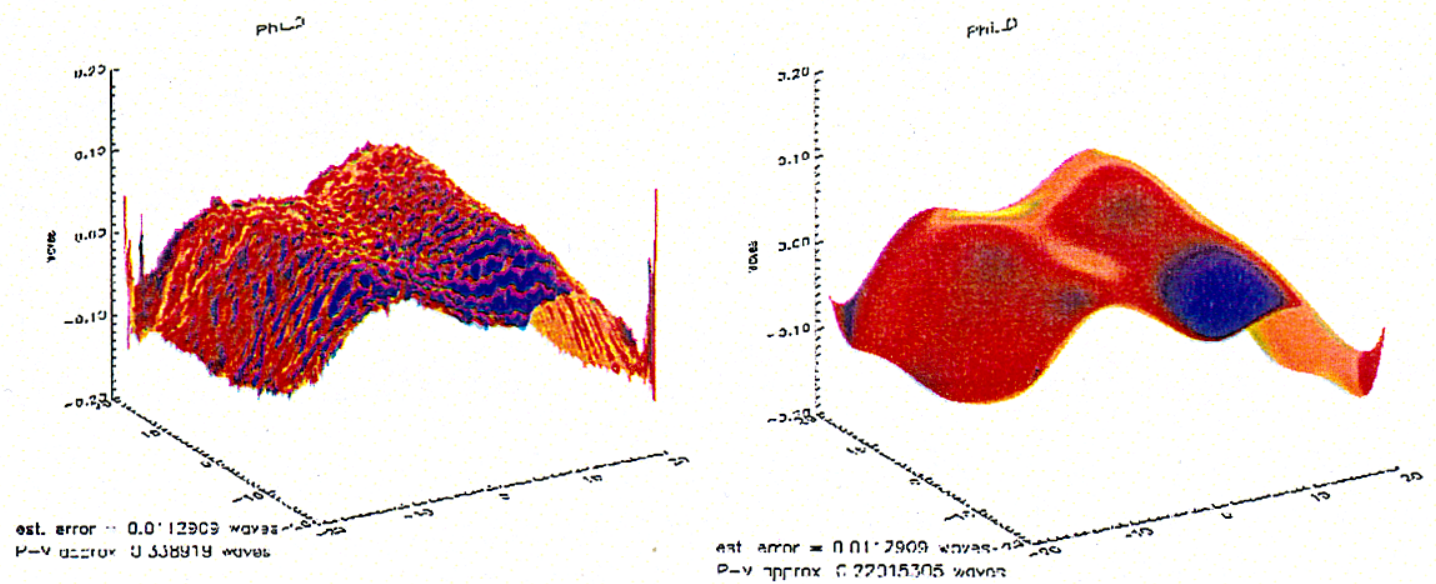

Figure 5.2. Measured phase distortion (and $6^{\text {th }}$ order polynomial fit) for diamond configuration (per slab/per pass). 

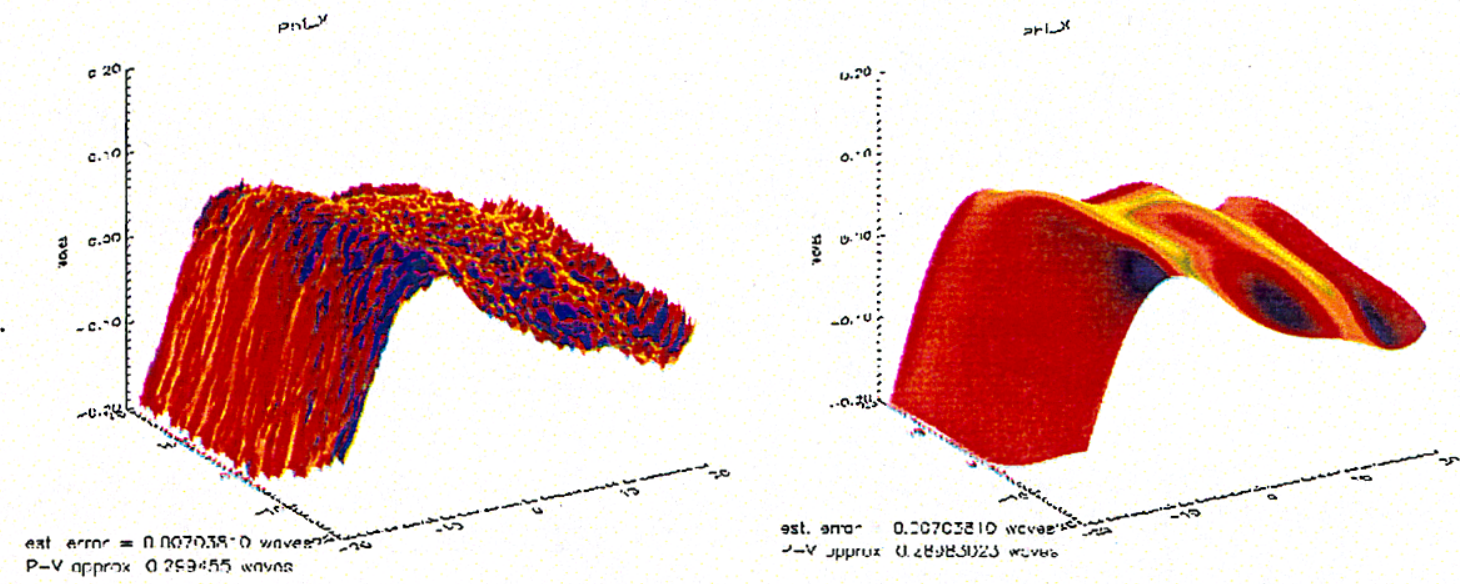

Figure 5.3. Measured phase distortion (and $6^{\text {th }}$ order polynomial fit) for $\mathrm{X}$ configuration (per slab/per pass).

The data show the expected general pattern produced by slab warping, with the phase retarded near the sides of the aperture relative to the middle, and with phase gradients larger in the horizontal direction than the vertical direction. Edge distortion, caused by the thermal expansion of the edge claddings as they absorb flashlamp light and ASE, is not evident in the data. Table 5.1 summarizes the results of these measurements, showing the $\mathrm{P}-\mathrm{V}$ value (for the sixth-order polynomial fits) and the rms gradient for the diamond, $\mathrm{X}$, and interior slab configurations.

Table 5.1. Measured results summary

\begin{tabular}{|l|c|c|l|}
\hline Configuration & $\begin{array}{c}\text { P-V distortion } \\
\text { (waves/slab/pass) }\end{array}$ & $\begin{array}{c}\text { Aperture-averaged rms } \\
\text { gradient }(\mathrm{nm} / \mathrm{cm})\end{array}$ & \multicolumn{1}{|c|}{ Data files } \\
\hline Diamond & $.22 \pm .01$ & 12.4 & $\begin{array}{l}\text { 0508C2/D2, 0508C4/D4, } \\
\text { 0508C6/D6 }\end{array}$ \\
\hline$X$ & $.29 \pm .01$ & 14.6 & $\begin{array}{l}\text { 0507C2/D2, 0507C4/D4, } \\
\text { 0507C6/D6 }\end{array}$ \\
\hline Interior & $.18 \pm .02$ & 7.9 & $\begin{array}{l}\text { 0505C2/D2, 0506C2/D2, } \\
\text { 0506C4/D4 (3-slab-long } \\
\text { data) }\end{array}$ \\
\hline
\end{tabular}

Measurements made at an explosion fraction of 0.23 were not statistically different from those made at an explosion fraction of 0.20 . These data exhibited an elevated level of background interference and were not formally included in the analysis set.

Additional measurements taken with the amplifier in the two-slab-wide $\mathrm{X}$ configuration showed wavefront distortion similar to the one-slab-wide $\mathrm{X}$ configuration. Differences in the distortions were small but statistically significant and presented 
themselves as saddle-shaped functions whose magnitude was twice that of the estimated experimental error.
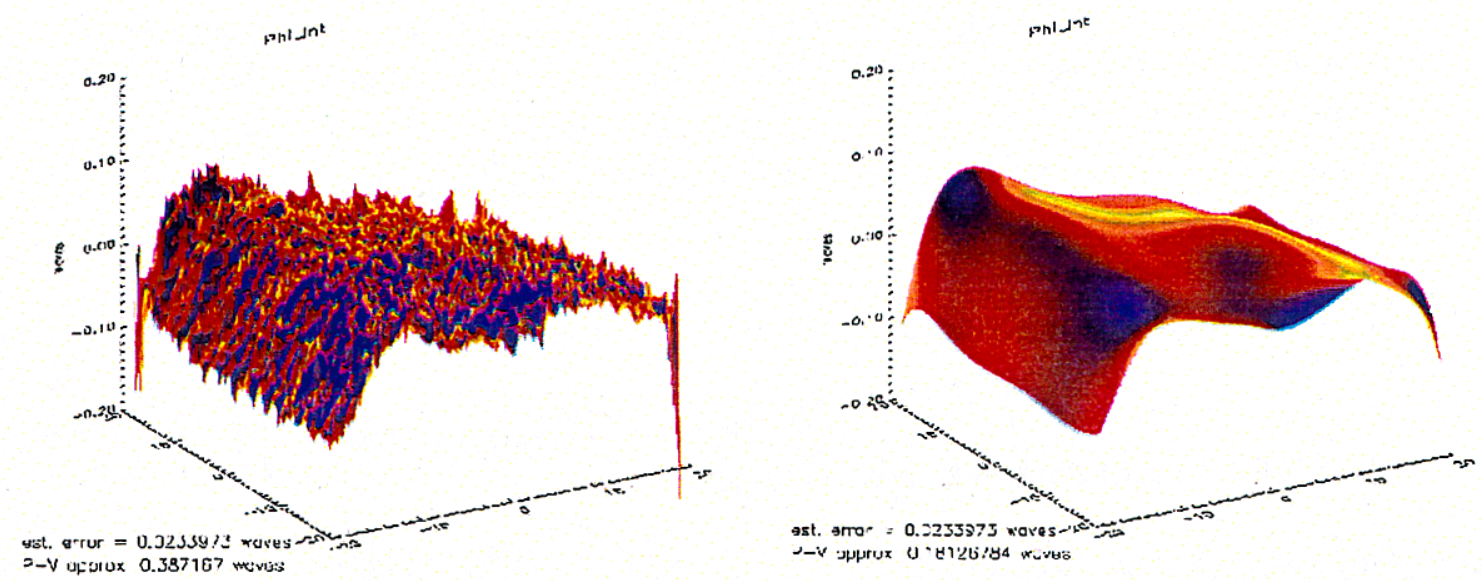

Figure 5.4. Measured phase distortion (and $6^{\text {th }}$ order polynomial fit) for interior configuration (per slab/per basis). Interior slab "measurement" was inferred from 3-log, diamond, and X measurements using Eq. 5.1.

\subsection{Pump-induced wavefront distortion model}

This section describes the model we currently use to calculate prompt pump-induced wavefront distortion of flashlamp-pumped, Brewster-angle slab amplifiers.

\section{Overview}

To calculate the wavefront distortion produced by flashlamp pumping processes, we used a suite of computer codes: Ampmodel [5.5], a 2D ray-trace code used to calculate the distribution of flashlamp pump light over the faces of the laser slabs; TOPAZ3D [5.6], to calculate the temperature distribution within the laser slab from pump distributions generated by Ampmodel; NIKE3D [5.7], to calculate the displacements and stresses from the temperature field given by TOPAZ3D; and OPL [5.8], which calculates the OPDs given the results from NIKE3D. The codes NIKE3D and TOPAZ3D are 3-dimensional finite-element analysis codes that have been in use at LLNL for over ten years. The optics code OPL is an in-house code based on the BREW code developed by Said Doss and Robert Gelinas.

\section{Temperature calculations}

We used a semi-empirical approach to calculate slab temperature rise, in which the thermal power density (in units of $\mathrm{W} / \mathrm{cm}^{3}$ ) is approximated with the calculated local $\mathrm{Nd}$-pumping rate multiplied by a scaling factor. The scaling factor was adjusted to give the best agreement between predicted and measured prompt pump-induced wavefront distributions. This "scaling" approach empirically handles several effects that could significantly affect the distribution of thermal power: depumping by amplified 
spontaneous emission, redistribution of energy by emission and reabsorption of $880-\mathrm{nm}$ fluorescence, absorption of ultraviolet and infrared radiation emitted by the flashlamps, and possible absorption of flashlamp light by excited Nd ions. Further, the Nd pumpingrate calculation did not expressly account for blue-shifting of the flashlamp emission as flashlamp electrical input power is increased. Thus, our method tends to underestimate wavefront distortion as flashlamp explosion fration is increased, or as flashlamp pulselength is decreased.

Appendix A provides a more detailed description of our method for calculating slab temperature rise.

\section{Determination of displacements and stresses}

The results of the slab temperature calculations were used in the code NIKE3D to calculate the resulting displacements and stresses. The displacements were small, on the order of $1 \mu \mathrm{m}$ and well within the linear elastic regime. Further, the temperature rise of the slab was only of order one degree Celsius. Therefore, we used a thermoelastic model with room temperature values for Poisson's Ratio, Young's Modulus, and the thermal expansion coefficient $[5.9,5.10]$.

Another important input to the NIKE3D code calculations was the boundary conditions. In the NIF prototype and production amplifiers, slabs rest on corrugated metal strips called Marcel springs. To simulate contact between the slab and the Marcel spring, the bottom nodes of the slab were merged to the top nodes of a strip of metal one element $(2.5 \mathrm{~mm})$ thick, while the bottom nodes of the metal strip were held fixed. The sides and top of the slab were allowed to move freely.

NIKE3D calculated the displacements and stresses as a function of time during the course of the pump pulse. Typically, the code was run only up to the time of peak gain, which is the time the probe beam was propagated through the prototype amplifier for most wavefront measurements.

\section{Determination of optical path differences (OPDs)}

To calculate the optical path length $L$ for rays passing through the slab, we used the code OPL, which integrated the equation

$$
L=\int n(x, y, z) d s,
$$

where $\mathrm{n}$ is the spatially varying refractive index and $\mathrm{s}$ is the distance along the ray path. There are two main sources of OPDs: (1) variations in path length caused by mechanical motion of the slab and (2) variations in path length caused by refractive index changes. 
The variations in path length caused by slab mechanical motion were estimated from the spatially varying displacements calculated in NIKE3D. Specifically, points $x, y$, and $z$ on the slab were translated according to the rules

$$
\begin{aligned}
& x \rightarrow x+u(x, y, z, t) \\
& y \rightarrow y+v(x, y, z, t), \\
& z \rightarrow z+w(x, y, z, t)
\end{aligned}
$$

where $u, v$, and $w$ were the displacements calculated with NIKE3D.

Two effects were taken into account to calculate the spatially varying refractive index: (1) the variation of refractive index with temperature and (2) the variation of refractive index with stress (stress-optic effect), i.e.,

$$
\mathrm{n}(\mathrm{x}, \mathrm{y}, \mathrm{z})=\mathrm{n}_{0}+(\mathrm{dn} / \mathrm{dT}) \Delta \mathrm{T}(\mathrm{x}, \mathrm{y}, \mathrm{z})+(\mathrm{dn} / \mathrm{d} \sigma) \Delta \sigma(\mathrm{x}, \mathrm{y}, \mathrm{z}),
$$

where we have symbolically written the change in refractive index due to stress as $(\mathrm{dn} / \mathrm{d} \sigma)$, and $\Delta \mathrm{T}$ and $\Delta \sigma$ are functions of time.

The sequence of events in calculating the OPD is as follows. The OPL code reads in the finite-element geometry from the NIKE3D output (plot) file. Each hexahedron finite element in the mesh is then broken up into six four-node tetrahedra. After OPL generates a connectivity matrix for these tetrahedra, Eq. (5.4) is used to calculate the refractive index at each node in the mesh. The refractive index is linearized within each tetrahedron according to

$$
n(x, y, z)=a+b x+c y+d z .
$$

The four unknowns in Eq. (5.5) are uniquely determined by the values of the refractive index at the four nodes of a given tetrahedron. With the refractive index linearized as in Eq. (5.5), we can then analytically solve the Eikonal equation [5.11] for the ray path within a tetrahedron

$$
\frac{d}{d s}\left(n \frac{d \bar{r}}{d s}\right)=\nabla n
$$

where $s$ is the distance along the ray path, and $r$ is the position vector of the ray. The connectivity matrix is used to determine which tetrahedra the ray enters and consequently the nodes at which the refractive index needs to be evaluated to calculate the unknowns in Eq. (5.5). We then track the ray as it propagates through the tetrahedra along the ray path, summing the optical path length as the ray propagates. 
In addition to calculating the optical path length, we can also calculate the depolarization a ray experiences as it propagates through the slab. We do this by calculating the Jones matrix [5.12] for each tetrahedron, which is assumed to act as a linear retarder. The final amount of retardation (and hence depolarization) is given by the product of all the individual Jones matrices for a given ray path.

\section{Example: AMPLAB diamond configuration}

Figure 5.5a shows the calculated OPD for the NIF prototype amplifier in the diamond configuration, while Figures $5.5 \mathrm{~b}, 5.5 \mathrm{c}$, and $5.5 \mathrm{~d}$ show the individual contributions from mechanical deformation, refractive index variations due to temperature variations, and refractive index variations due to stress, respectively. Comparison of Fig. 5.5a with Fig.5.5b shows that mechanical deformation accounts for nearly all the calculated wavefront distortion. Figures $5.5 \mathrm{c}$ and $5.5 \mathrm{~d}$ confirm that refractive index variations due to temperature and stress are relatively minor contributors to the overall OPD.

Fig. 5.6 shows the $\mathrm{P}$ to $\mathrm{S}$ depolarization (on a per-slab basis) for the AMPLAB diamond configuration. As expected, the greatest amount of depolarization occurs in the corners, where the two pieces of edge cladding meet; it is there where the greatest amount of stress occurs. Note the similarity of the stress distribution in Fig. 5.5d and depolarization distribution in Fig. 5.6. The overall depolarization is small, well within its specification of less than $0.05 \%$ averaged over the aperture.

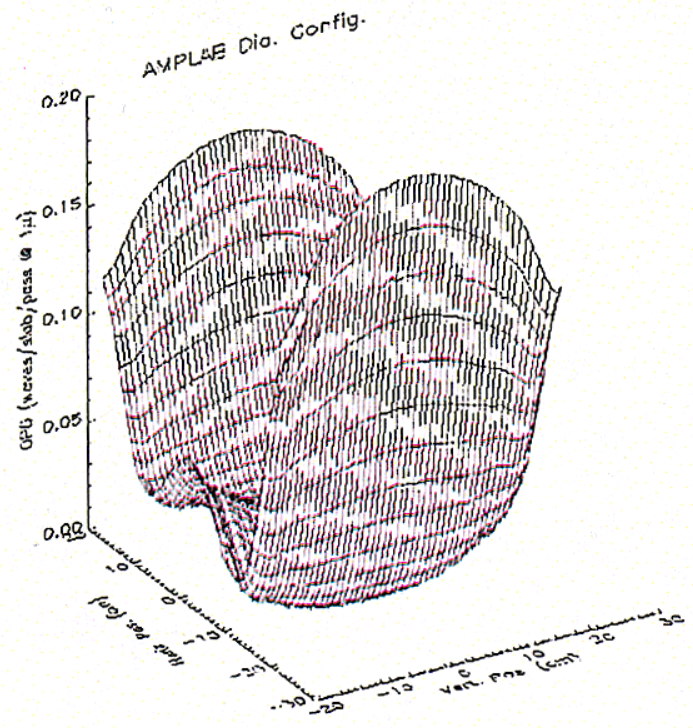

(a)

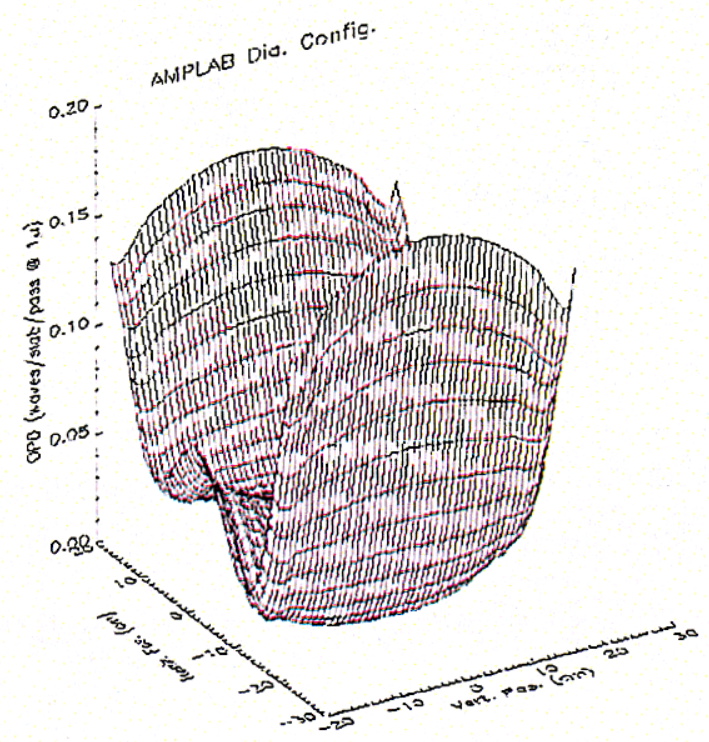

(b)

Figure 5.5 (a) and (b). OPD calculated for the AMPLAB diamond configuration, $f_{x}=0.2$ : (a) all effects, (b) displacement effects only. 
ANPLAB Dir. Config.

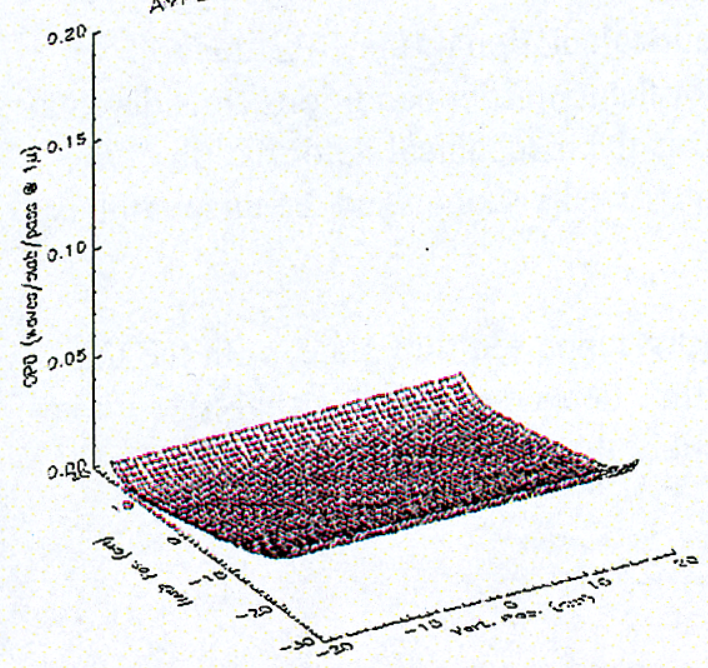

(c)

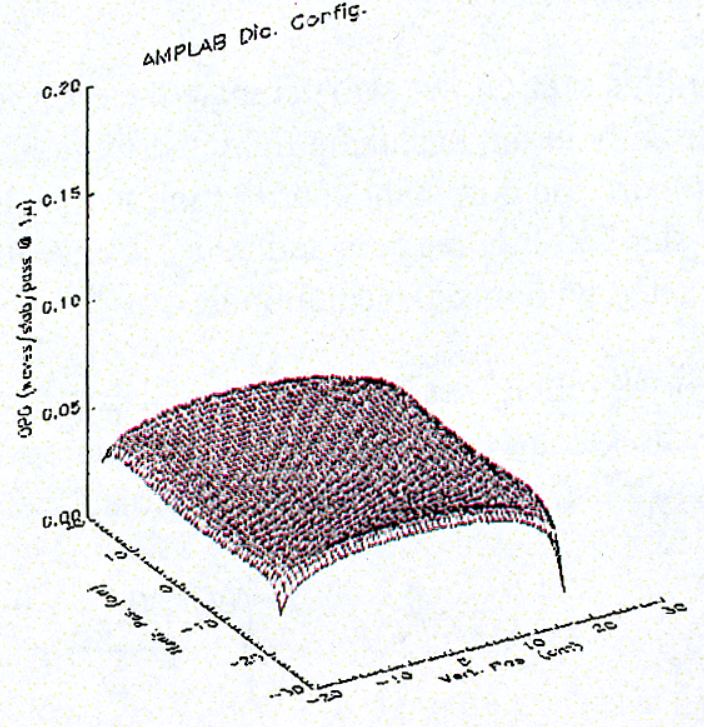

(d)

Figure 5.5 (cont.). OPD calculated for the AMPLAB diamond configuration, $f_{x}=0.2:(c) d n / d T$ effects only and (d) stress effects only.

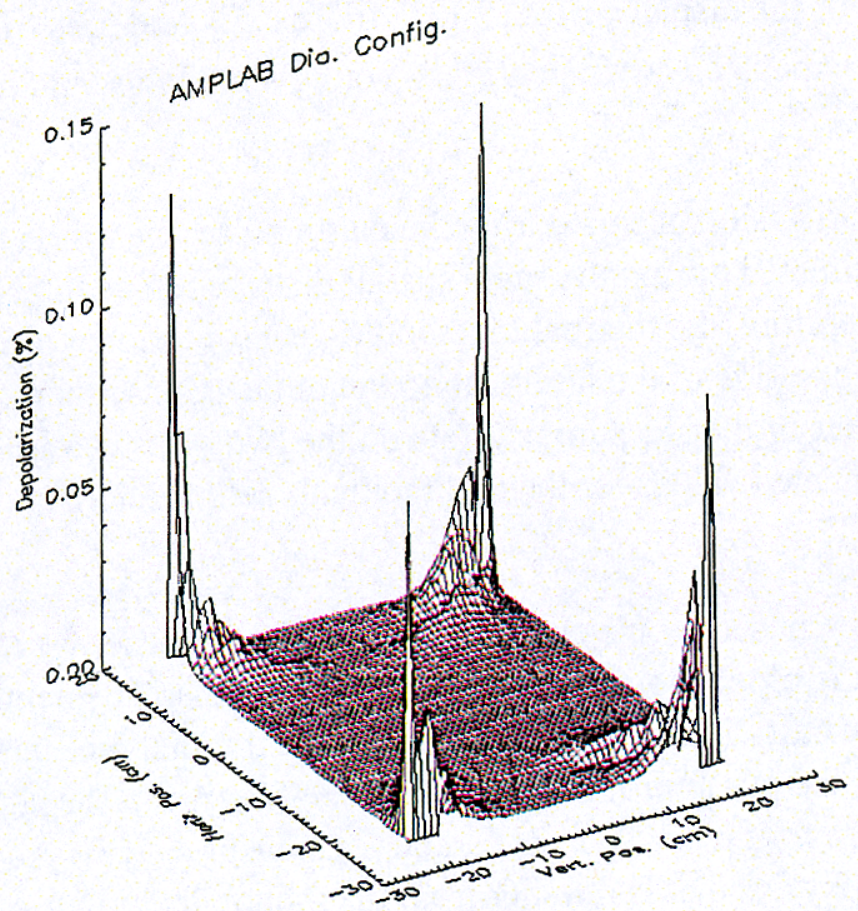

Figure 5.6 Depolarization calculated for the AMPLAB diamond configuration, $f_{x}=\mathbf{0 . 2}$.

79 


\section{Error analysis}

In this section, we will estimate the error in our calculation of the OPD due to uncertainty in the flashlamp light distribution. For the amplifier conditions considered in this report, the dominant contribution to the OPD is the mechanical deformation of the laser slab (cf. Figures 5.5a and 5.5b). Consequently, we have analyzed the uncertainties associated with mechanical motion.

For simplicity, assume the laser slab is a simply supported thin plate, with the thin dimension along the z-axis. We will neglect any time dependence in this analysis. It may be shown that the equation for $w$, the displacement in the $\mathrm{z}$-direction, is given by

$$
\nabla^{2} w(x, y)=-\left(\frac{M_{T}(x, y)}{1-\mathbf{v}}\right)
$$

where the thermal moment, defined as

$$
M_{T}(x, y)=\alpha E \int_{0}^{h}(z-h / 2) T(x, y, z) d z
$$

is the source function for the displacement [5.13]. In Eqs. 5.7 and 5.8, $v$ is Poisson's Ratio, $\alpha$ is the thermal expansion coefficient, $E$ is Young's modulus, and $h$ is the slab thickness.

Since little thermal diffusion occurs during the pump pulse, the driving term $T(x, y, z)$ in Eq. 5.12 was approximated during the same spatial distribution as the $\mathrm{Nd}$ pumping rate as described above. Therefore, the thermal moment $M_{T}$ is proportional to the difference in Nd-pumping rate from one side of the laser slab to the other. Consequently, small uncertainties in the values of the Nd-pumping rate on the two sides can lead to large uncertainties in the calculated values for thermal moment, deformation, and wavefront distortion.

To estimate the error caused by uncertainties in the pump profile, we make use of the fact that the phase front is proportional to the gradient of the displacement. From Eq. (5.7), we see that this quantity is simply the integral of the thermal moment, given by Eq. (5.8). If we assume that the pump profiles can vary by $+-5 \%$, it can be shown that the variation in the P-V value for the phase front can be as much as $+-25 \%$. Additional uncertainty arises from physics that is missing from the model, including ASE decay, fluorescence redistribution, ultraviolet and infrared absorption, and flashlamp spectral shifts as noted earlier. 


\subsection{Comparison of model predictions with AMPLAB measurements}

This section presents for comparison both model predictions with wavefront measurements made on the NIF prototype amplifier in AMPLAB. Unless noted otherwise, the AMPLAB measurements were performed in the third aperture from the top (the " $\mathrm{C}$ " aperture) with the flashlamps fired at an explosion fraction of 0.2. Also, all model predictions were made using the same value of the parameter $\mathrm{A}$, used to scale the thermal power density.

Figures 5.7-5.9 show 3D plots of the predicted and measured wavefronts for the diamond, $\mathrm{X}$, and interior slabs respectively. The predicted wavefronts have nearly the same shapes as the measured wavefronts, which were fit to sixth-order polynomials as described above. A 3D plot of the differences between the measured and calculated wavefronts are shown in Figures 5.10 and 5.11 for the diamond and X configurations respectively. The rms aperture-averaged difference is .012 waves for the diamond configuration and .021 waves for the $\mathrm{X}$ configuration, less than $8 \%$ of the $\mathrm{P}-\mathrm{V}$ value in the aperture.

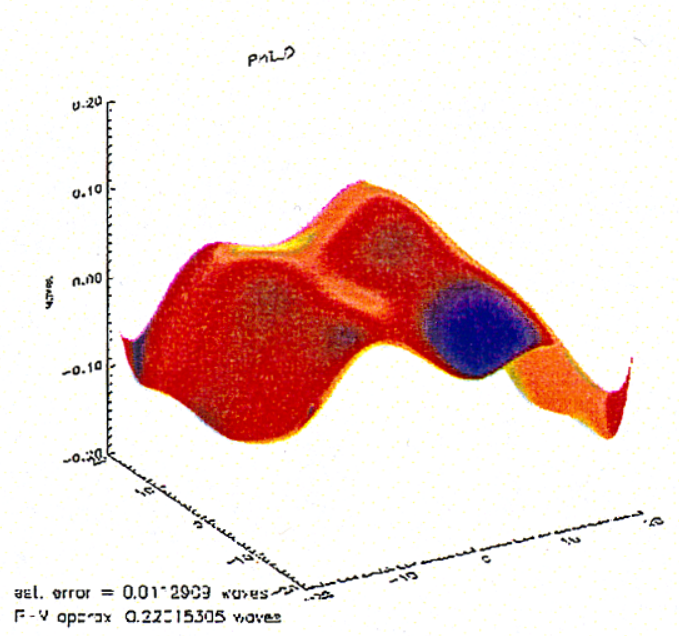

\section{Diamond configuration}

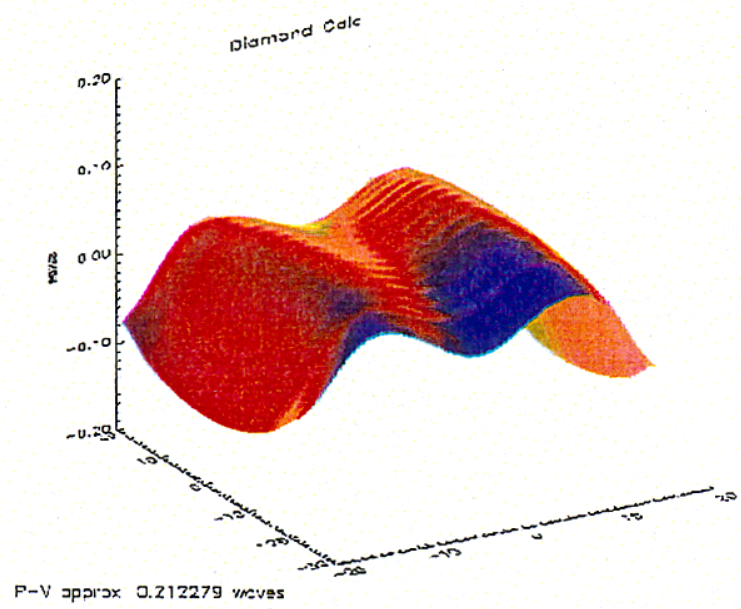

Figure 5.7. Measured (upper left) and calculated (lower right) wavefront for the AMPLAB diamond configuration. 


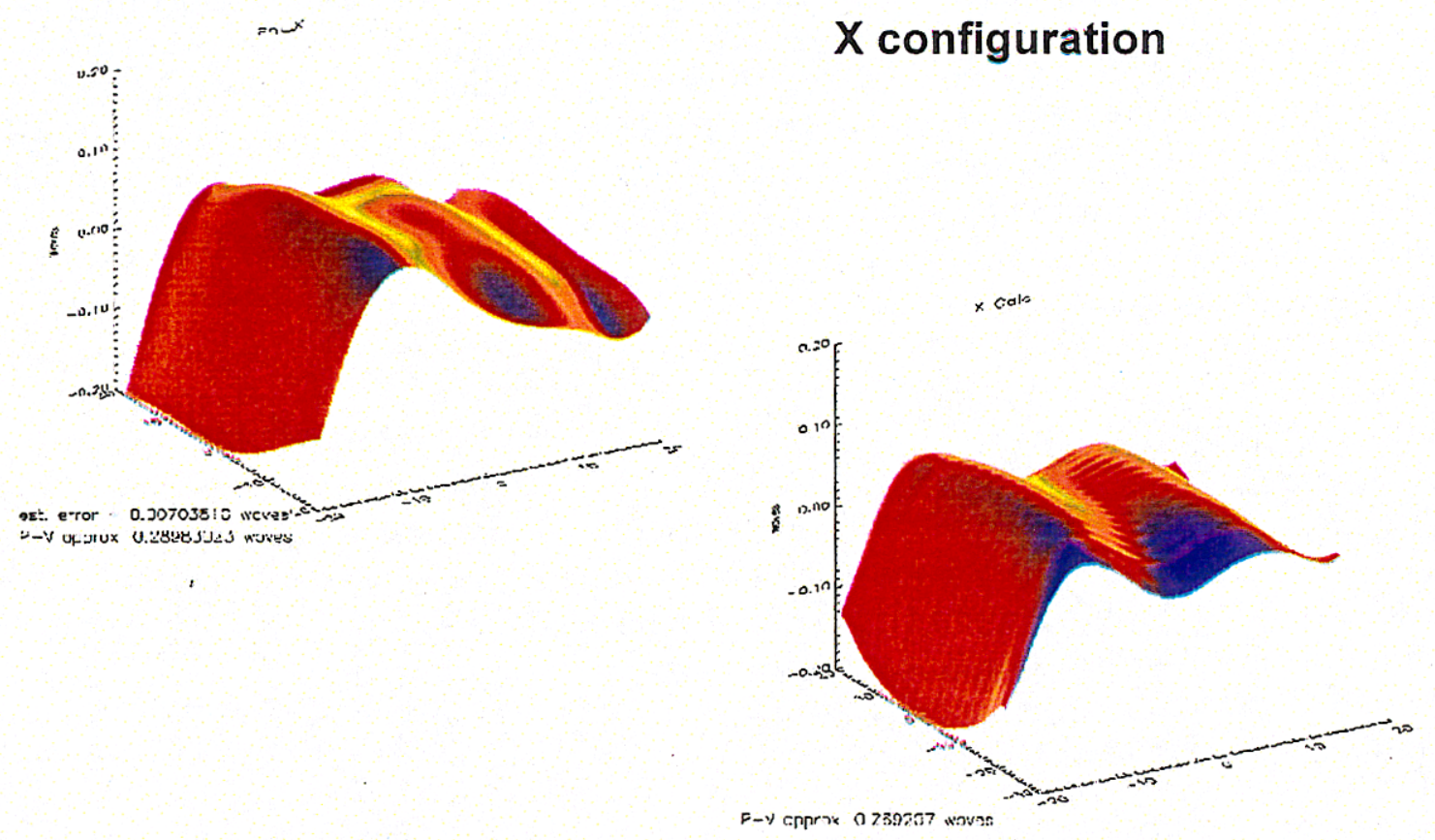

Figure 5.8 Measured (upper left) and calculated (lower right) wavefront for the AMPLAB X configuration.

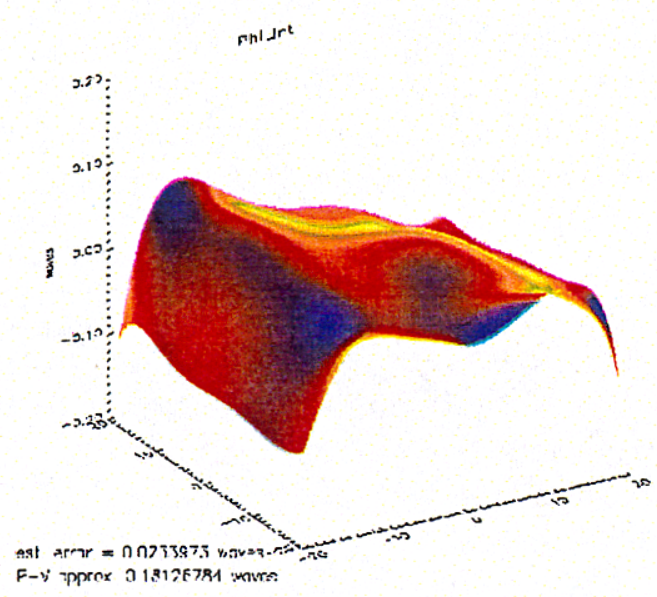

*Interior slab "measurement" inferred from 3-long, diamond and $X$ measurements according to formula:

$$
\Phi_{\text {int }}=\Phi_{\text {3-long,meas }} / 2-\left(\Phi_{\text {diamond,meas }}+\Phi_{x, \text { meas }}\right) / 4
$$

\section{Interior slab}

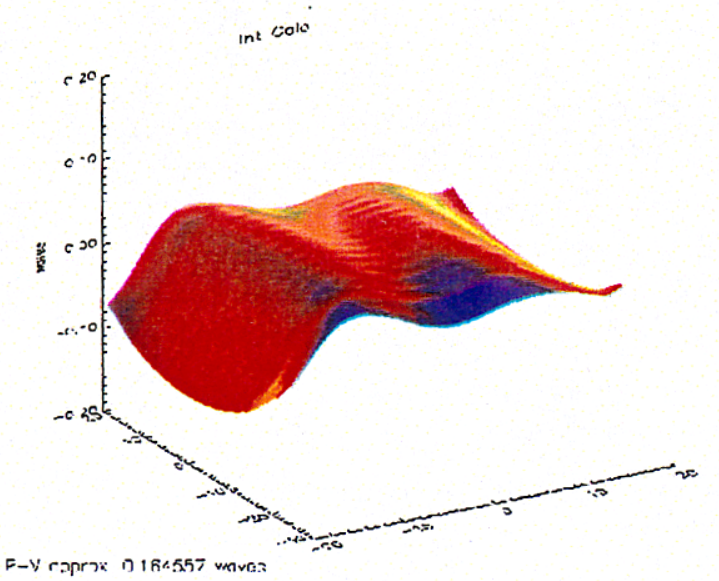

Figure 5.9. Measured (upper left) and calculated (lower right) wavefront for the AMPLAB interior configuration. 


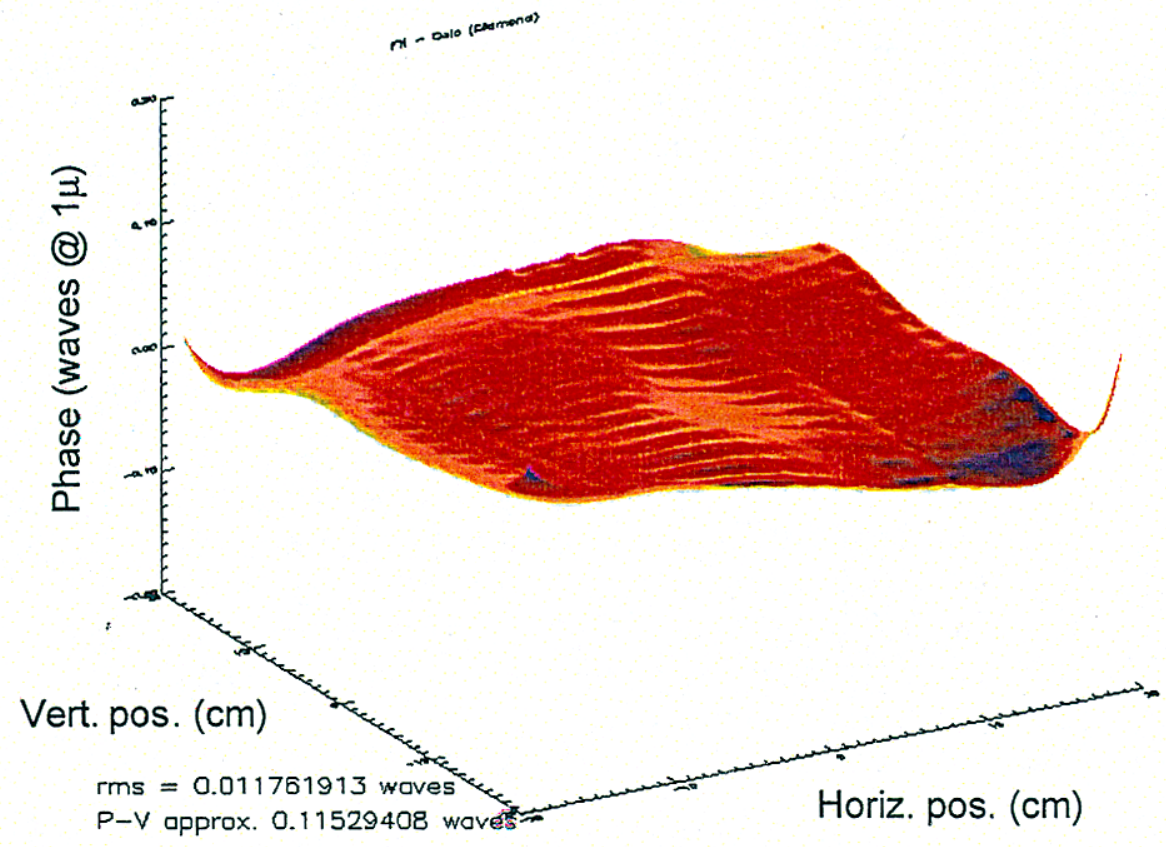

Figure 5.10. Difference between calculated and measured wavefront: AMPLAB diamond configuration.

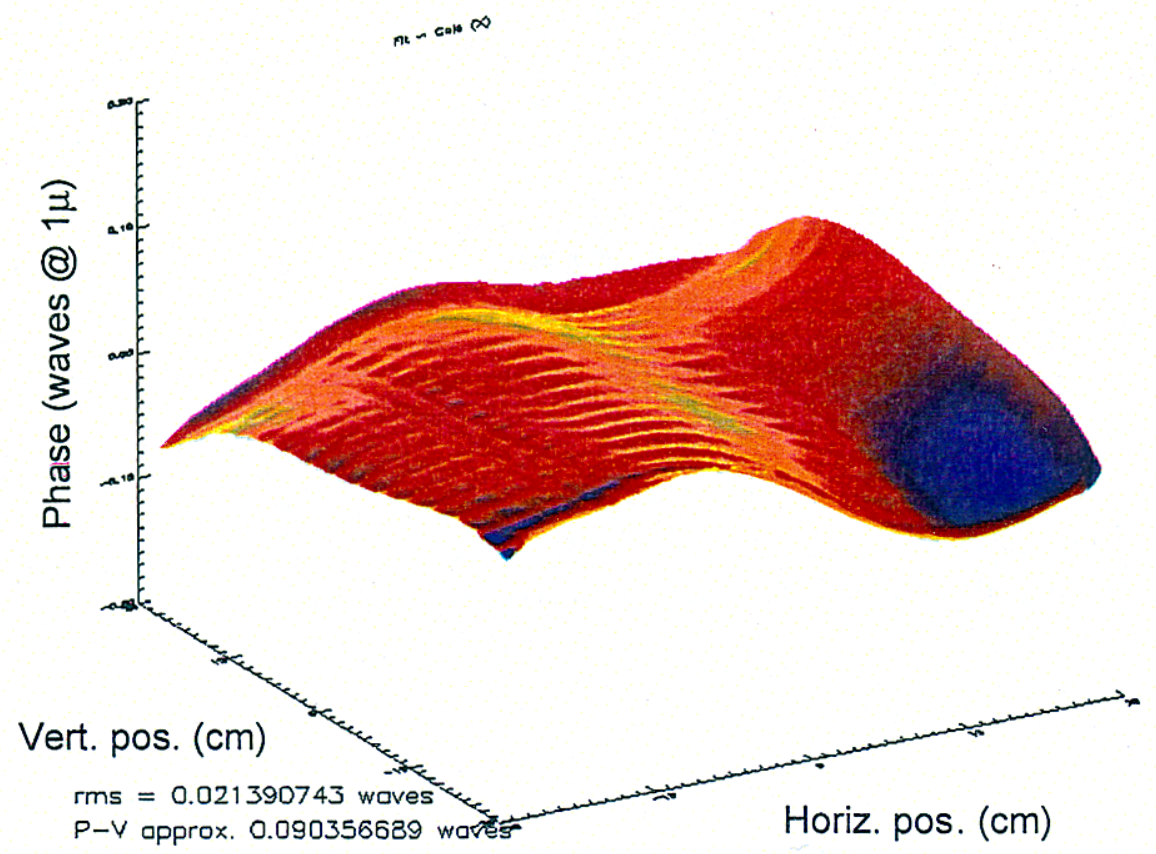

Figure 5.11. Difference between calculated and measured wavefront: AMPLAB X configuration. 
Horizontal and vertical line-outs allow differences between predictions and measurements to be seen more readily than in 3D plots. Figures 5.12 (a), (b), (c), and (d) show predicted and measured phase fronts vs horizontal position for the diamond, X, 3-slab-long, and interior configurations, respectively. In Figure 5.13 (a), (b), and (c), we show the results for the predicted and measured phase fronts vs vertical position for the diamond, $\mathrm{X}$, and interior configurations respectively. In all four cases, the predictions agree with the measurements within the experimental error, which was estimated from the aperture-averaged rms variation evaluated over three shots. A summary of the measured and calculated results is shown in Table 5.2.

Table 5.2. AMPLAB Measured/Calculated Summary

\begin{tabular}{|l|c|c|}
\hline Configuration & $\begin{array}{c}\text { Measured P-V } \\
\text { wavefront distortion } \\
\text { (waves/slab/pass) }\end{array}$ & $\begin{array}{c}\text { Calculated P-V wavefront } \\
\text { distortion (for <LG-770) } \\
\text { (waves/slab/pass) }\end{array}$ \\
\hline Diamond & $.22 \pm .03$ & $.21 \pm .03$ \\
\hline $\mathrm{X}$ & $.29 \pm .03$ & $.27 \pm .04$ \\
\hline Interior & $.18 \pm .03$ & $.16 \pm .02$ \\
\hline
\end{tabular}

AMPLAB - Diamond Config.

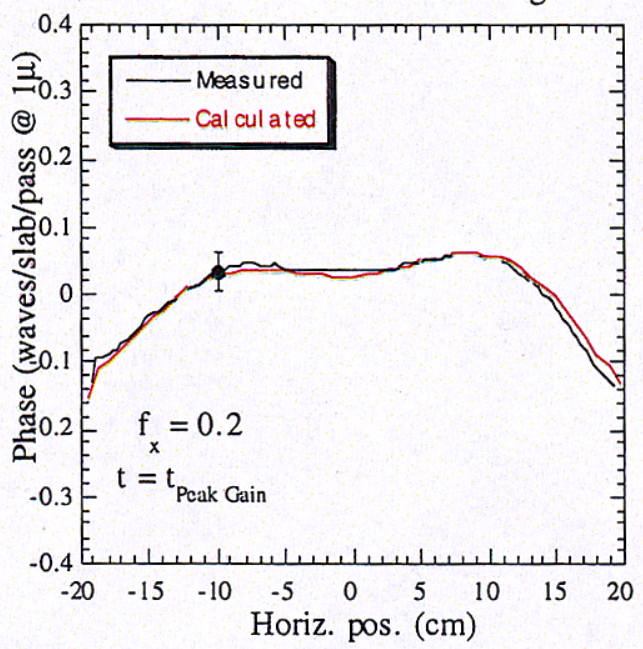

(a)

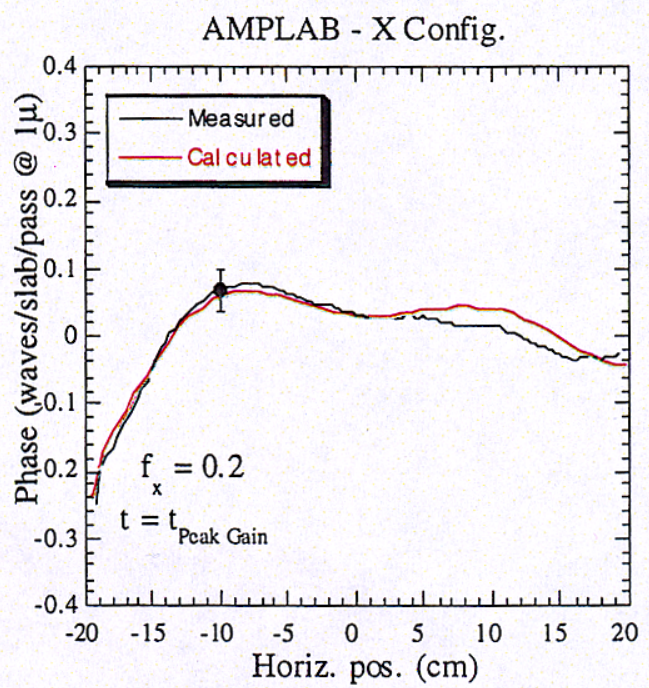

(b)

Figure 5.12. Measured and calculated wavefront, horizontal component-AMPLAB: (a) diamond configuration, (b) $\mathrm{X}$ configuration. Error bar shows typical error for measurement. 
AMPLAB - 3-long config.

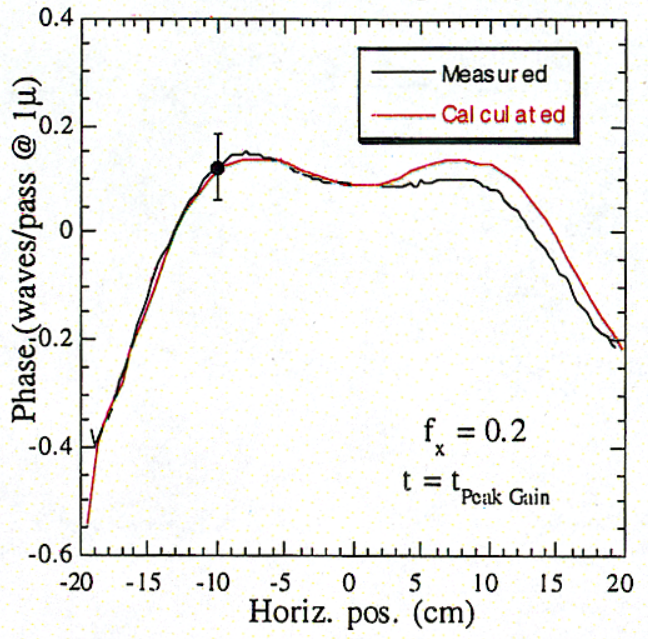

(c)

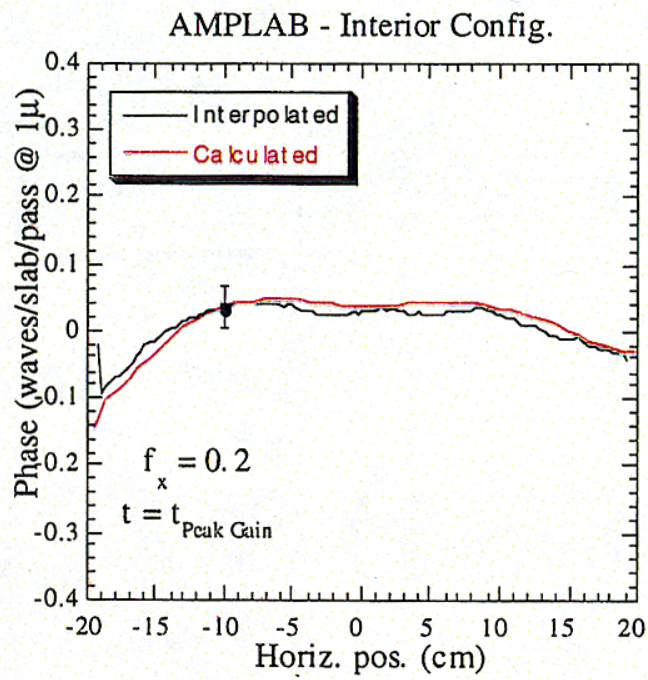

(d)

Figure 5.12 (cont.). Measured and calculated wavefront, horizontal component-AMPLAB: (c) 3-slab-long configuration, and (d) interior configuration-interpolated from 3-long, diamond, and $X$ results.

AMPLAB - Diamond Config.

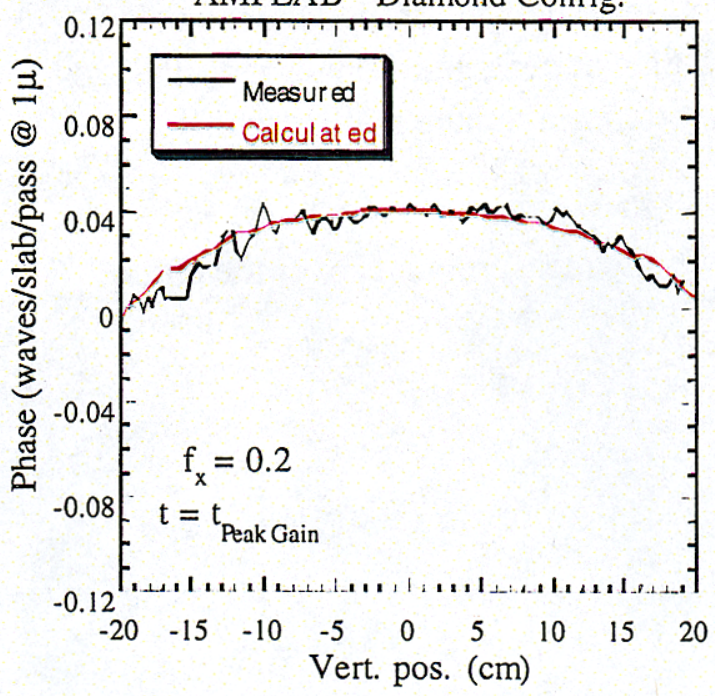

(a)

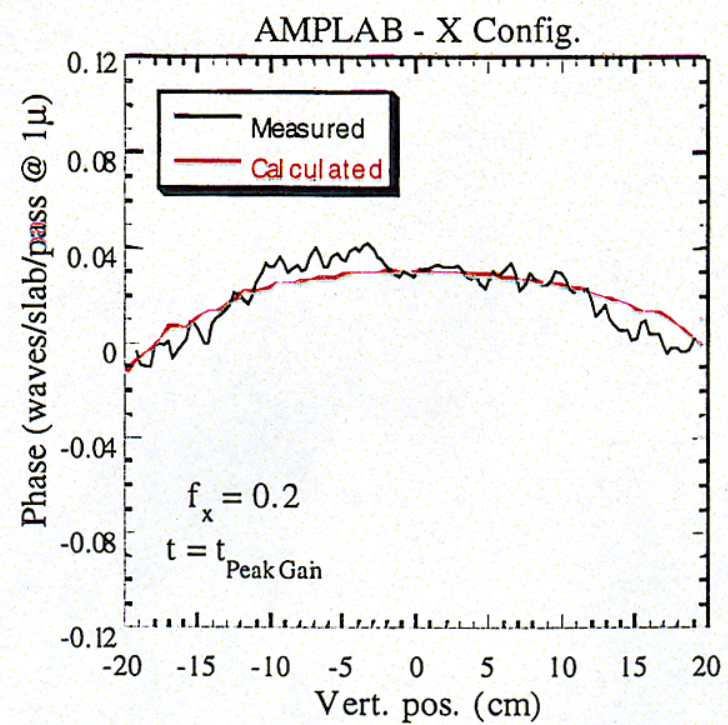

(b)

Figure 5.13. Measured can calculated wavefront vs vertical position-AMPLAB (a) diamond configuration and (b) $\mathrm{X}$ configuration. Line-outs taken at horizontal midplane. 


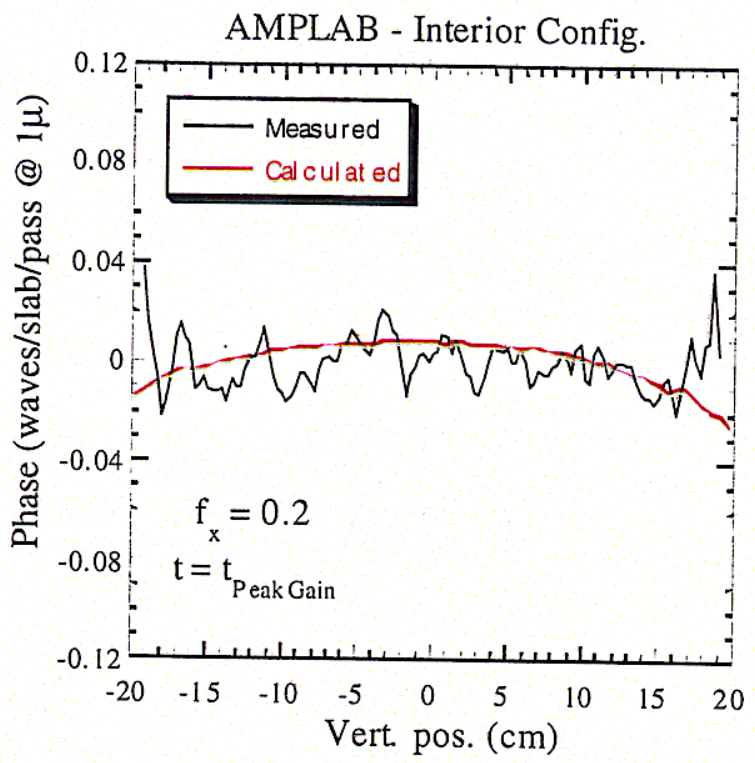

(c)

Figure 5.13 (cont.). Measured can calculated wavefront vs vertical position-AMPLAB (c) interior configuration. Line-outs taken at horizontal midplane.

We also compared the model predictions with wavefront measurements made at various times $(100,200,300$, and $500 \mu$ s) after the time of peak gain. See Figures 5.14 (a)-(e). This series of graphs shows the growth of wavefront distortion as the slabs continue to expand and distort after the flashlamp pulse. The wavefront distortion (P-V) was about three times greater $500 \mu$ s after peak gain than at peak gain. The shape change reflects the fact that several vibrational modes with different fundamental frequencies were excited.

The model predictions are in good agreement with the measurements over the entire time studied. 


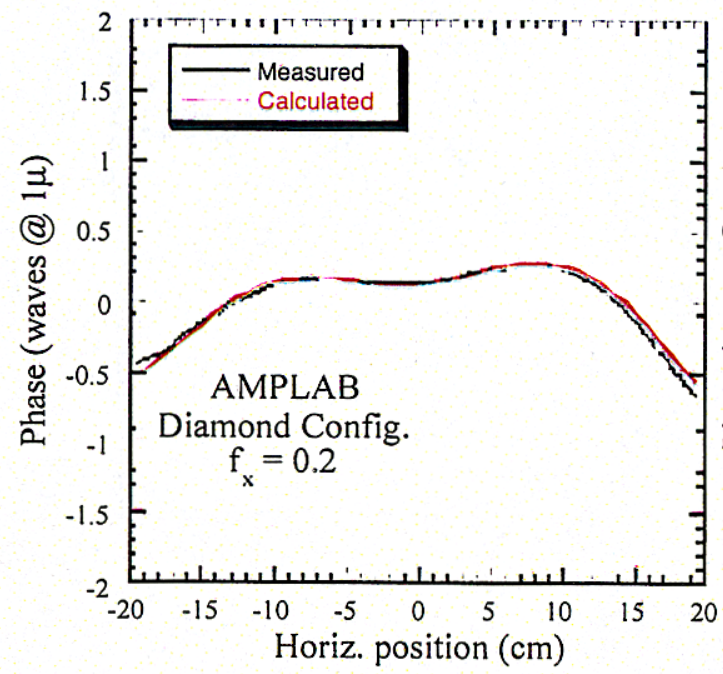

(a)

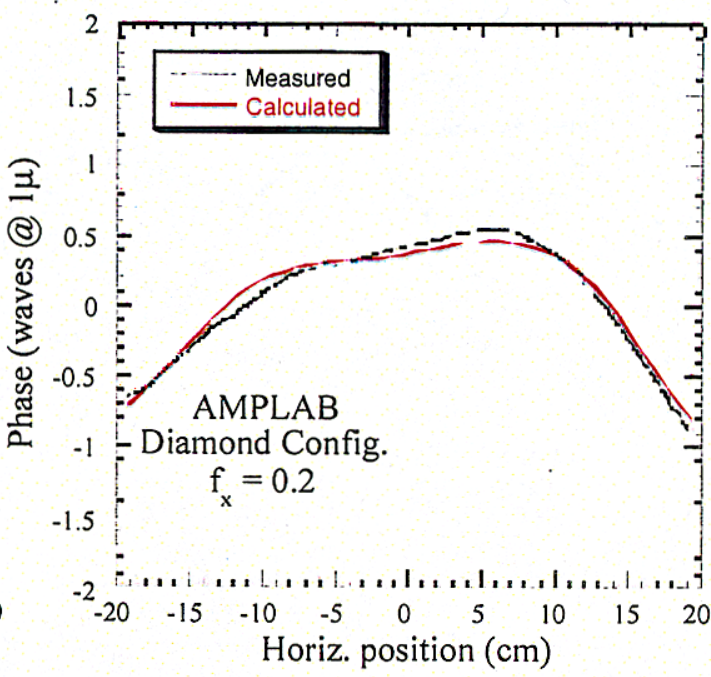

(b)

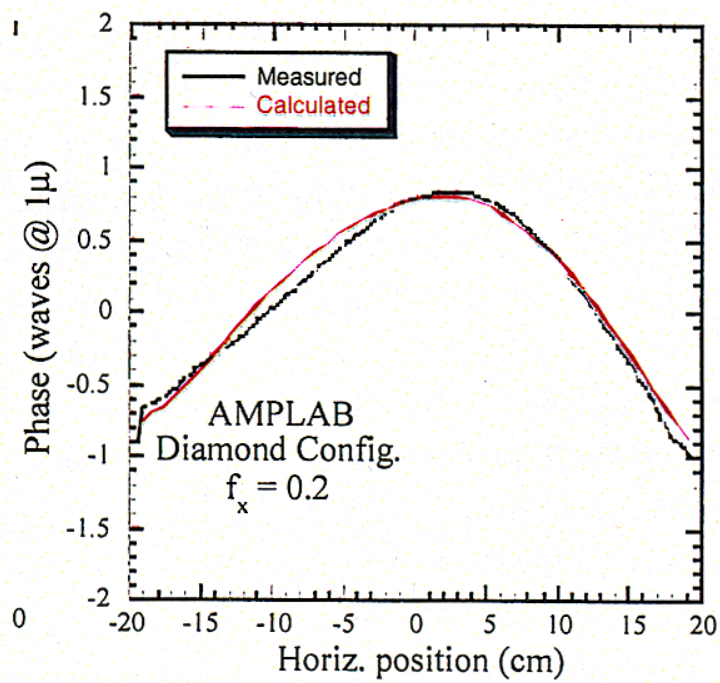

(c)

Figure 5.14. Measured and calculated wavefront versus horizontal for the AMPLAB, diamond configuration: measurements taken at (a) $t=t_{\text {penk gain }}$, (b) $t=t_{\text {peak gain }}+0.1 \mathrm{~ms}$, and (c) $t=t_{\text {peak gain }}+$ $0.1 \mathrm{~ms}$ and (d) $\mathrm{t}=\mathrm{t}_{\text {peak gain }}+0.2 \mathrm{~ms}$. 


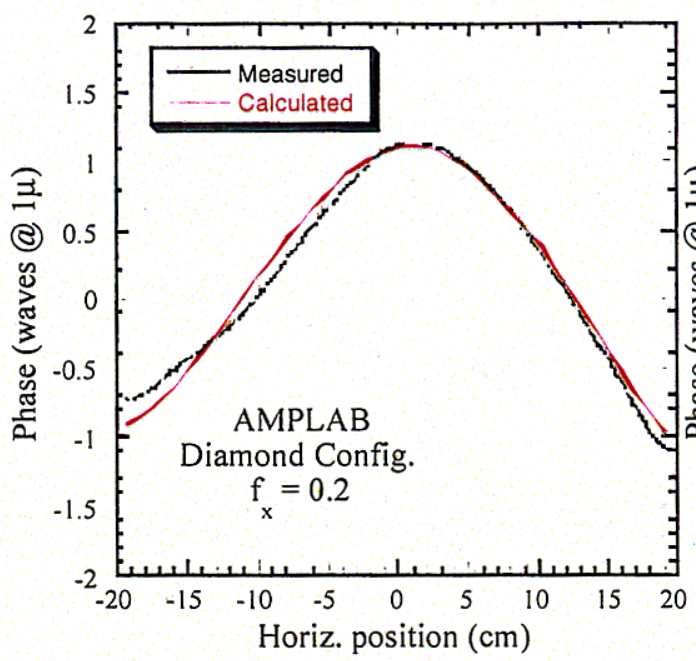

(d)

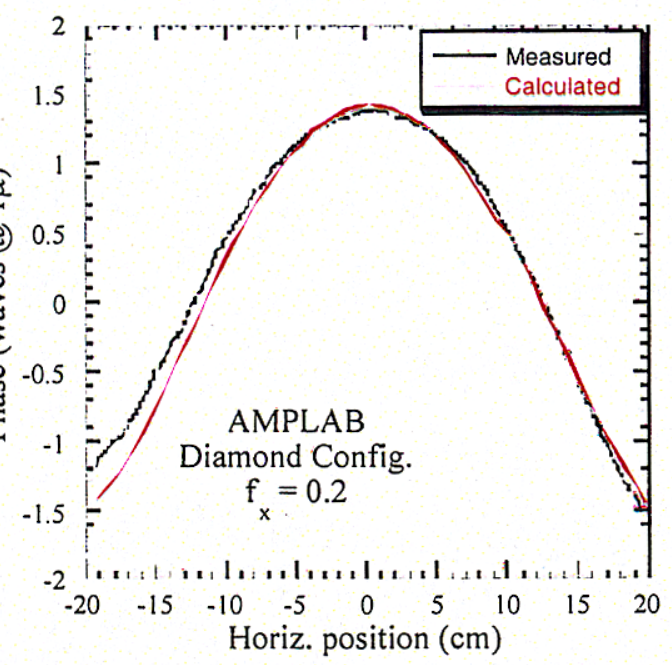

(e)

Figure 5.14 (cont.). Measured and calculated wavefront vs. horizontal position for the AMPLAB diamond configuration: measurements taken at (d) $) t=t_{\text {peak gain }}+0.3 \mathrm{~ms}$ and (e) $t=t_{\text {peak gain }}+0.5 \mathrm{~ms}$.

\subsection{Comparison of model predictions with Beamlet measurements}

Wegner et al. used a full-aperture radial shear interferometer to measure prompt pump-induced wavefront distortion produced by the entire Beamlet multipassed chain [5.15]. Figures 5.15 and 5.16 show horizontal and vertical line-outs, respectively, for the predicted and measured phase. The modeling took into account the Beamlet amplifier reflector shapes, numbers of flashlamps, flashlamp bore diameter, flashlamp pulselength (except for blue-shifting, as described above), and laser-glass proportion. The source-term scaling factor "A" (see Appendix A) used for the Beamlet predictions was set at the same value used for AMPLAB modeling. The P-V magniturde of the predicted wavefront is consistent with the measured wavefront when the estimated \pm 0.1 -wave experimental uncertainty is taken into account. It is noteworthy that the AMPLAB and Beamlet data can be modeled using the same scaling factor, offering greater confidence in its magnitude. However, it is perplexing why the predicted and measured wavefronts have significant differences for Beamlet, while there are such small differences for AMPLAB. Errors in the predicted pump profiles could account for a large fraction of the discrepancy. Another notion is that measurements were affected by surface distortions induced by flashlamp light. 
Beamlet system wavefront - horiz. component

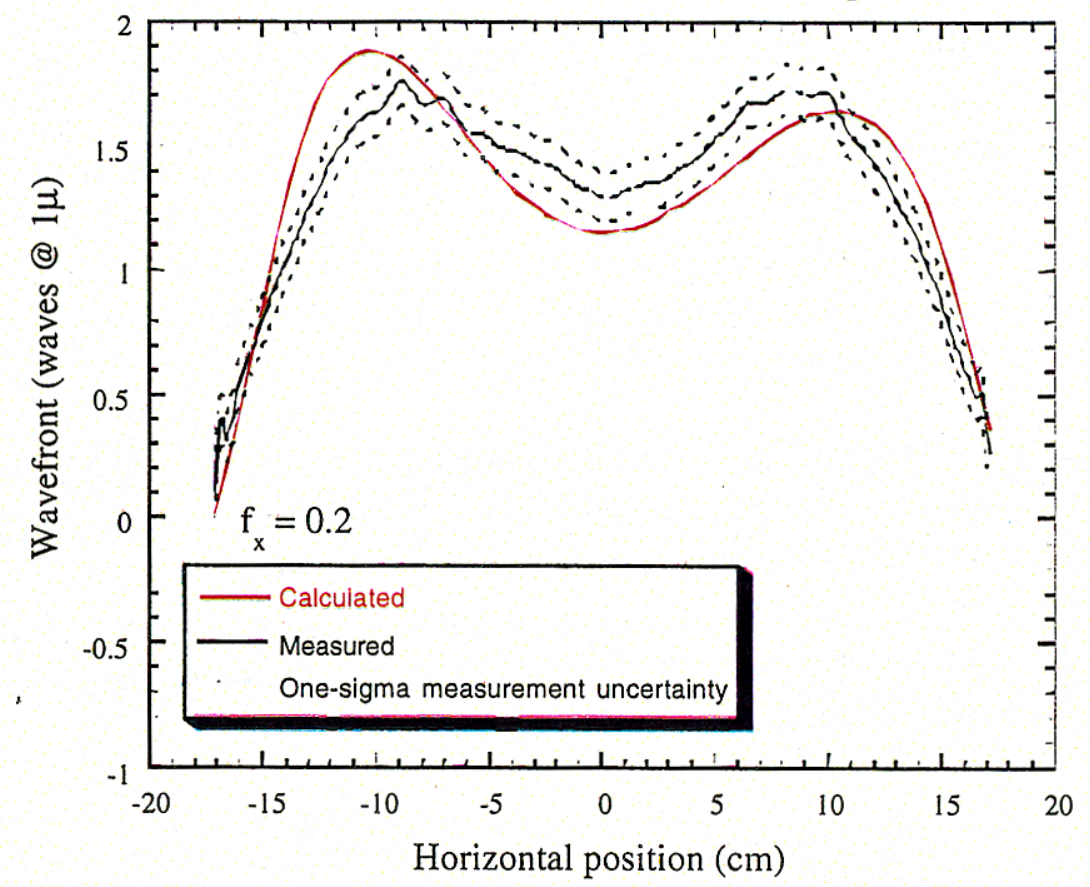

Figure 5.15. Measured and calculated Beamlet system wavefront-horizontal component. Calculated values use an AMPLAB-based source-term scaling factor.

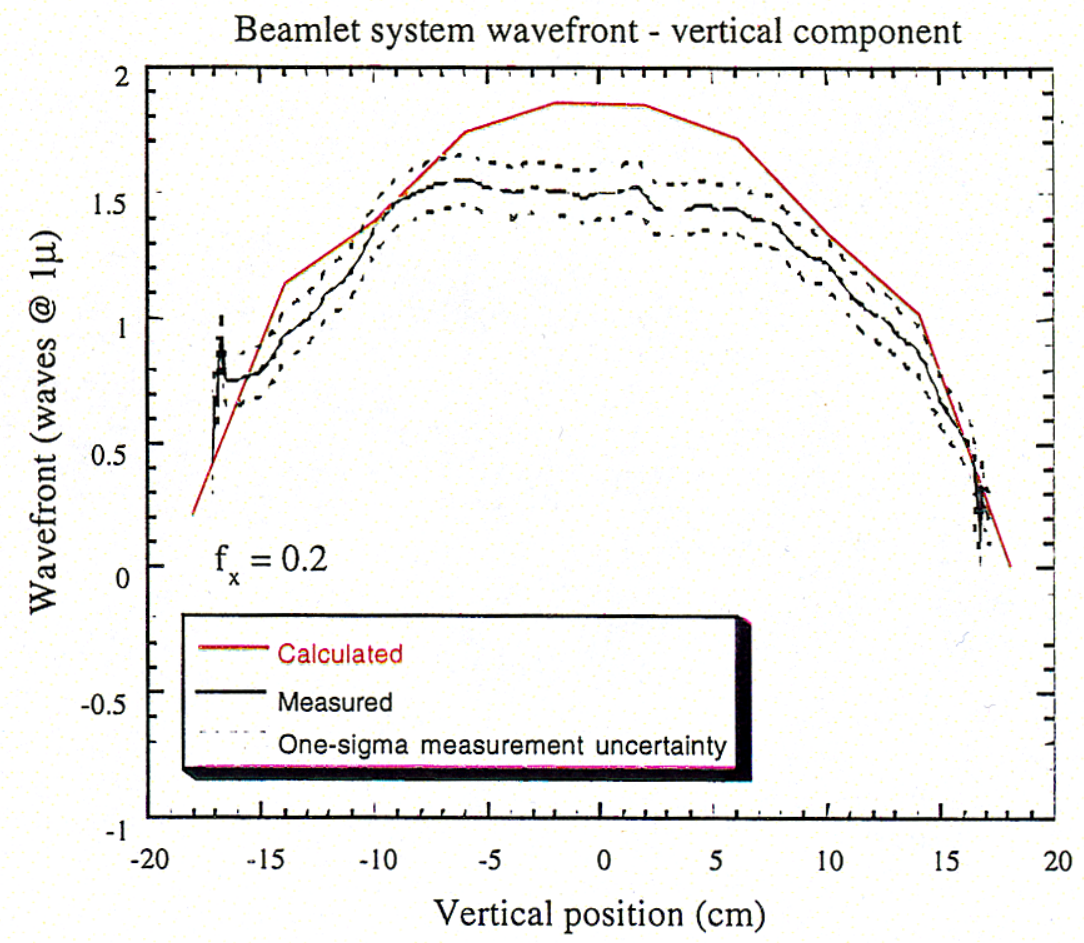

Figure 5.16. Measured and calculated Beamlet system wavefront-vertical component. Calculated values use a source-term scaling factor based on AMPLAB measurements. 


\subsection{Predictions for NIF}

With the model calibrated to the AMPLAB measurements, we predicted the prompt pump-induced wavefront distortion for a NIF laser beam. We ran our models for both LHG-8 and LG-770 laser glass using NIF baseline flashlamp energy, flashlamp pulselength, and reflector geometries. The difference in performance between the two glasses is small: a system composed entirely of LG-770 would have a P-V wavefront distortion about $10 \%$ greater than a system composed entirely of LHG-8. This difference is due to the different material constants between LHG-8 and LG-770 [5.10].

Figures 5.17 (a) and (b) show the predicted wavefront distortion for the diamond and $\mathrm{X}$ configuration, respectively. The results shown are on a per slab/pass basis. In Figures 5.18 (a) and (b), we show the results for the interior configuration and for the entire system (50/50 mix of LHG-8 and LG-770 laser glasses). The system wavefront was calculated for the 11-0-5 (baseline) configuration taking into account the image flip in going from the booster amplifiers to the cavity amplifiers.

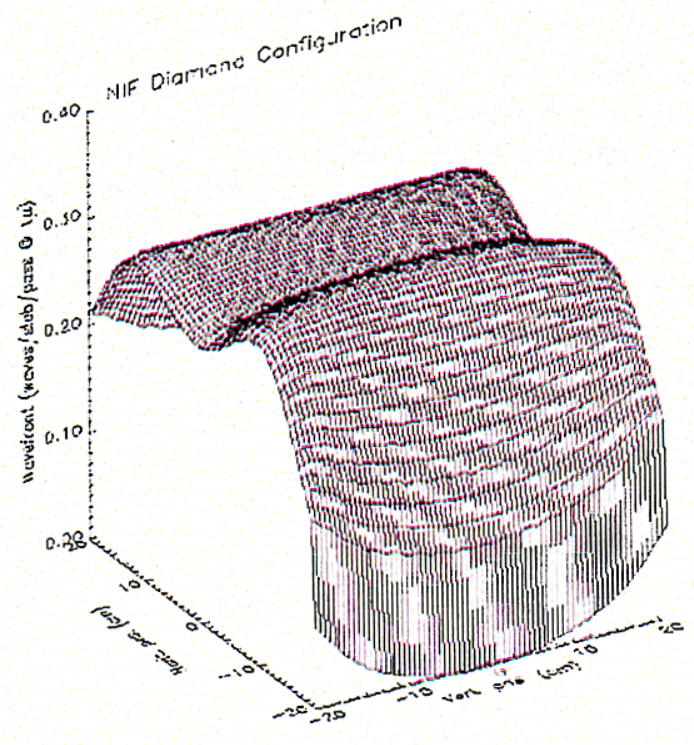

(a)

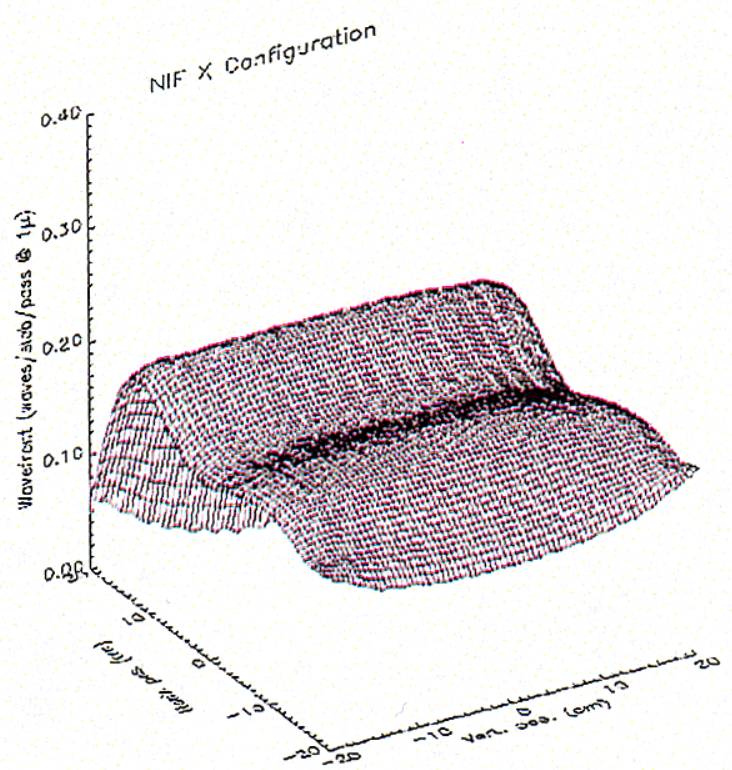

(D)

Figure 5.17. NIF wavefront predictions at $f_{x}=0.2$ for LG-770, for laser glass: (a) diamond configuration and (b) $\mathrm{X}$ configuration. 


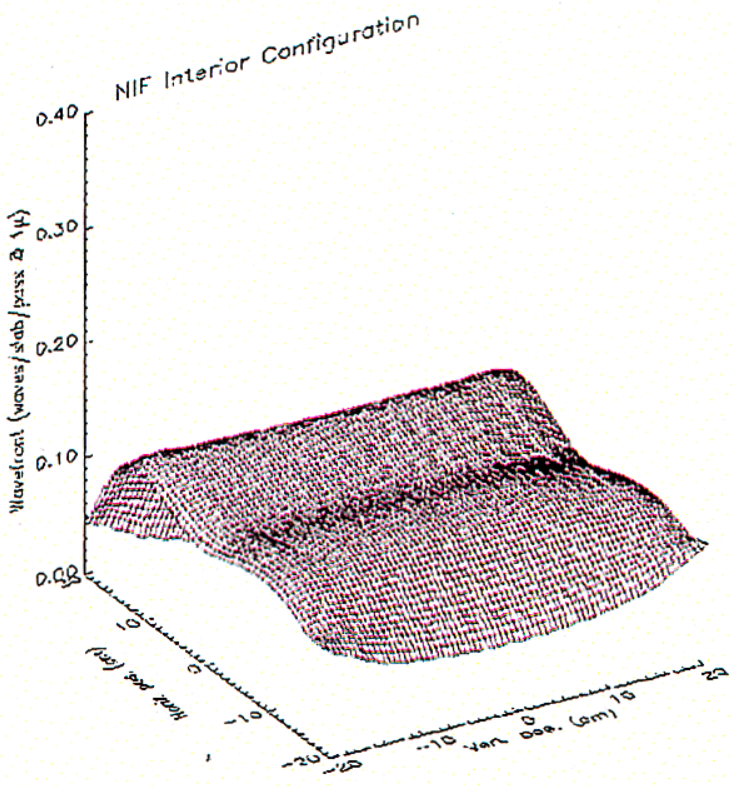

(a)

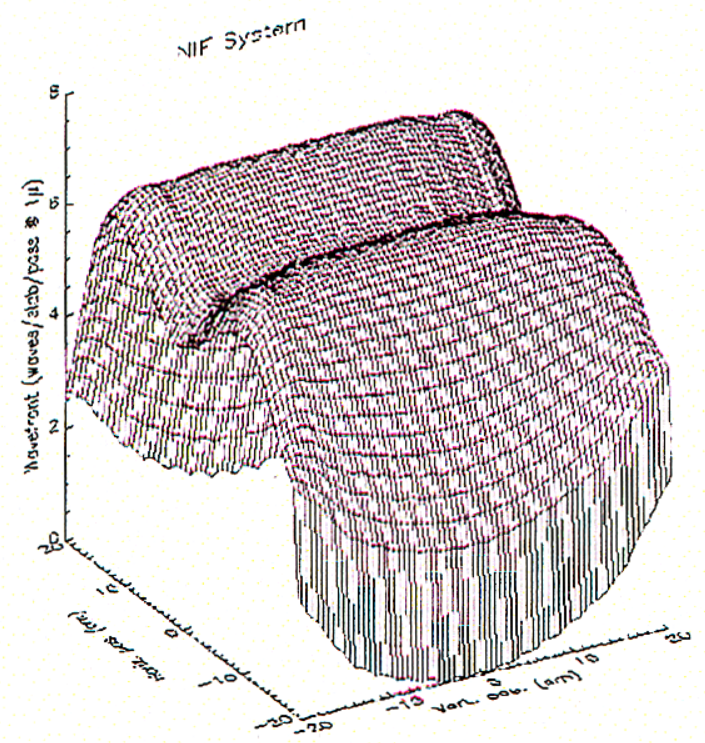

(b)

Figure 5.18. NIF wavefront predictions at $f_{x}=0.2$, (a) interior configuration (LG-770 laser glass) and (b) system (50/50 LHG-8/LG-770 laser glass).

In Figures 5.19 and 5.20, we show the predicted wavefront vs horizontal position at the vertical midplane, corresponding to the results shown in Figures 5.17 and 5.18. For comparison, the calculated AMPLAB results are plotted as well. The wavefronts are similar in $\mathrm{P}-\mathrm{V}$, but there are slight differences in shape, especially near the side array (on the left side of the plot in the figure). These differences can be traced to the differences in pump cavity design between the NIF and AMPLAB. Figures 5.21 and 5.22 show a plan view of the pump cavity for AMPLAB and NIF respectively. As indicated on Figure 5.22 , the central and side flashlamp arrays for the NIF are displaced by $1 \mathrm{~cm}$ in the direction parallel to the beam. Further, the target points (indicated by lines emanating from the flashlamps) are different for the NIF geometry, especially for the central array. These differences result in different reflector shapes and consequently slightly different pump profiles.(see Figure 5.23). Figure 5.24 shows the predicted wavefront vs vertical position at the horizontal midplane. The wavefront is slightly asymmetric about the midplane due to the fact that the slab sets on one of its faces and consequently not all surfaces are free. This effect was noticed in the Beamlet results [cf. Figure 5.16] and was taken into account in our model by adjusting the boundary conditions, as described above. The wavefront distortion is approximately 6.0 waves for the horizontal lineout and 1.0 waves for the vertical line-out not taking into account vignetting and beam size. 


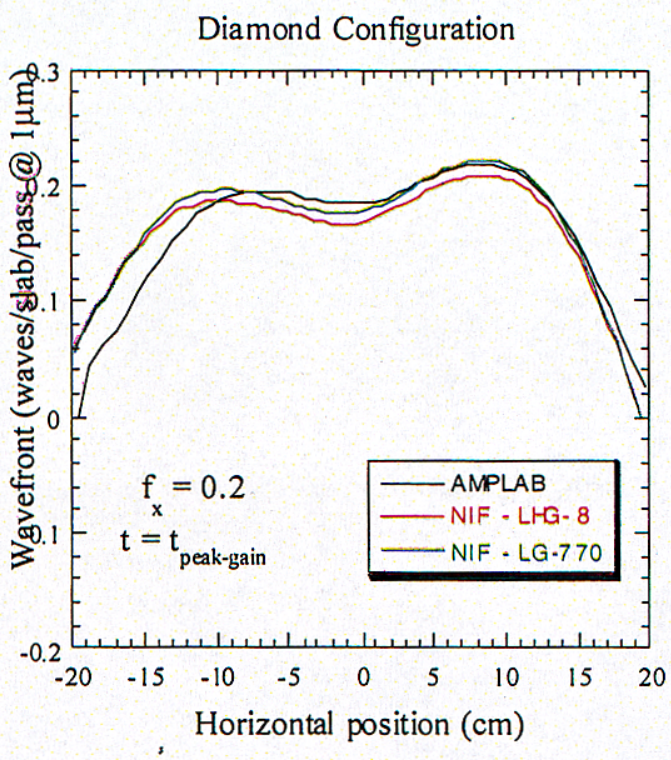

(a)

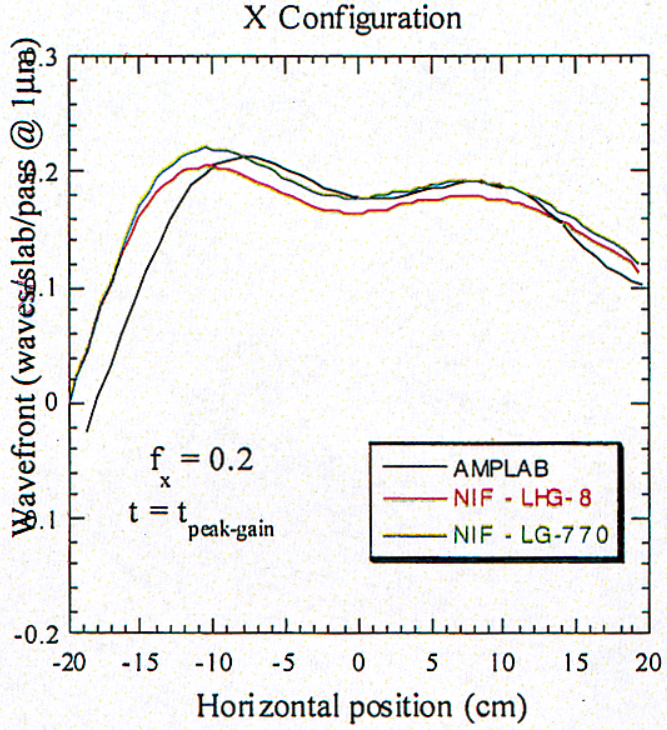

(b)

Figure 5.19. NIF wavefront predictions, horizontal line-out at vertical midplane for two laser glasses: (a) diamond configuration and (b) $\mathrm{X}$ configuration. Also shown for comparison are the AMPLAB calculations.

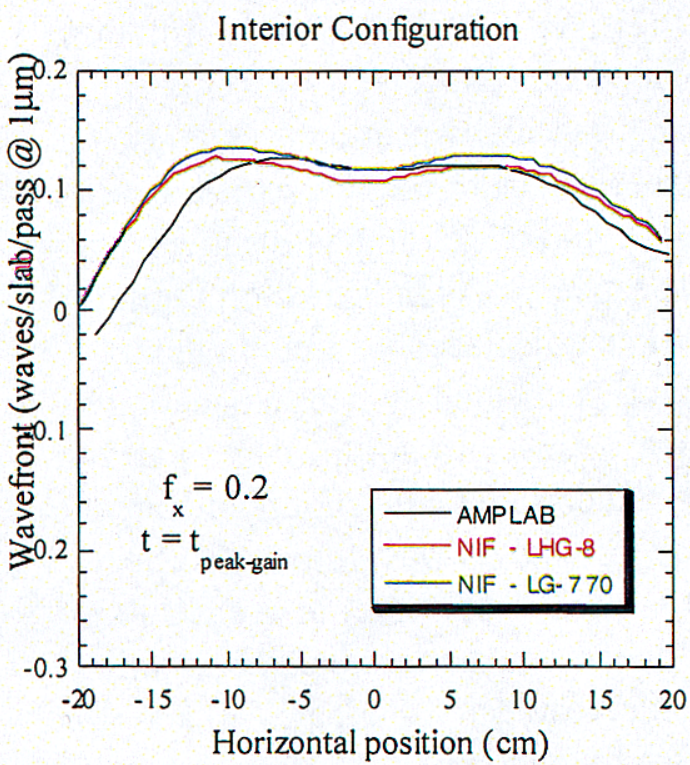

(a)

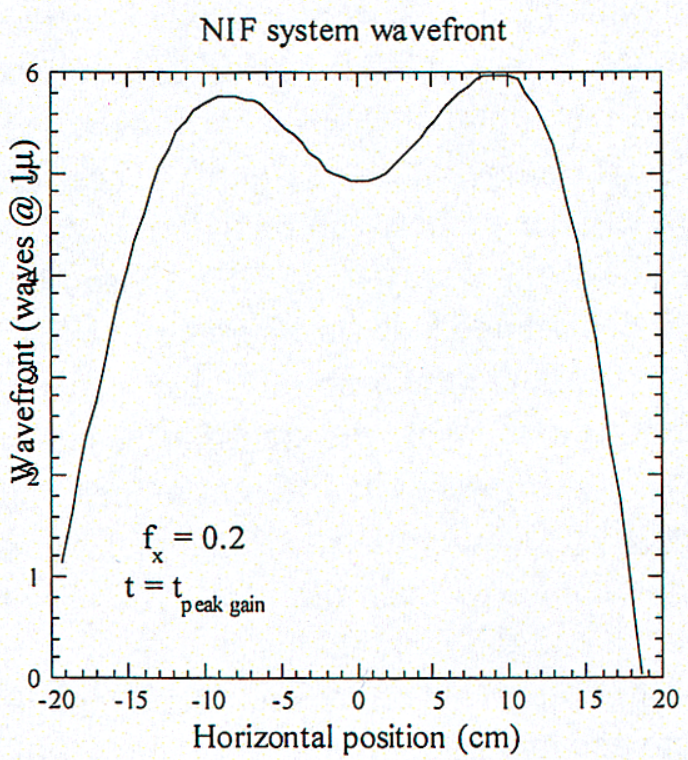

(b)

Figure 5.20. NIF wavefront predictions, horizontal line-out at vertical midplane: (a) interior configuration with AMPLAB calculations for comparison and (b) system (50/50 LHG-8/LG-770 laser glass). 


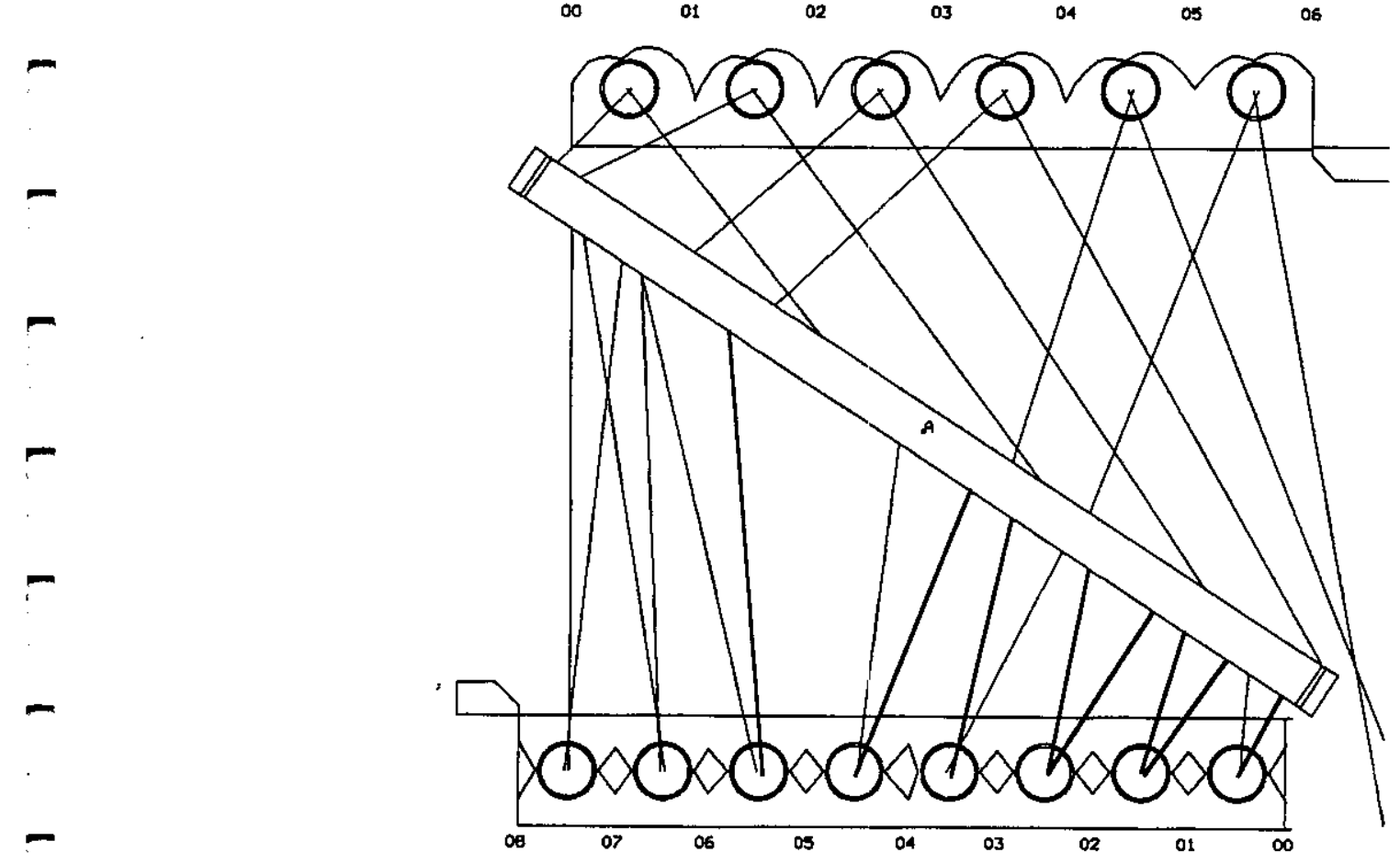

Figure 5.21. Plan view of AMPLAB pump cavity. Lines indicate target points on slab from a given lamp.
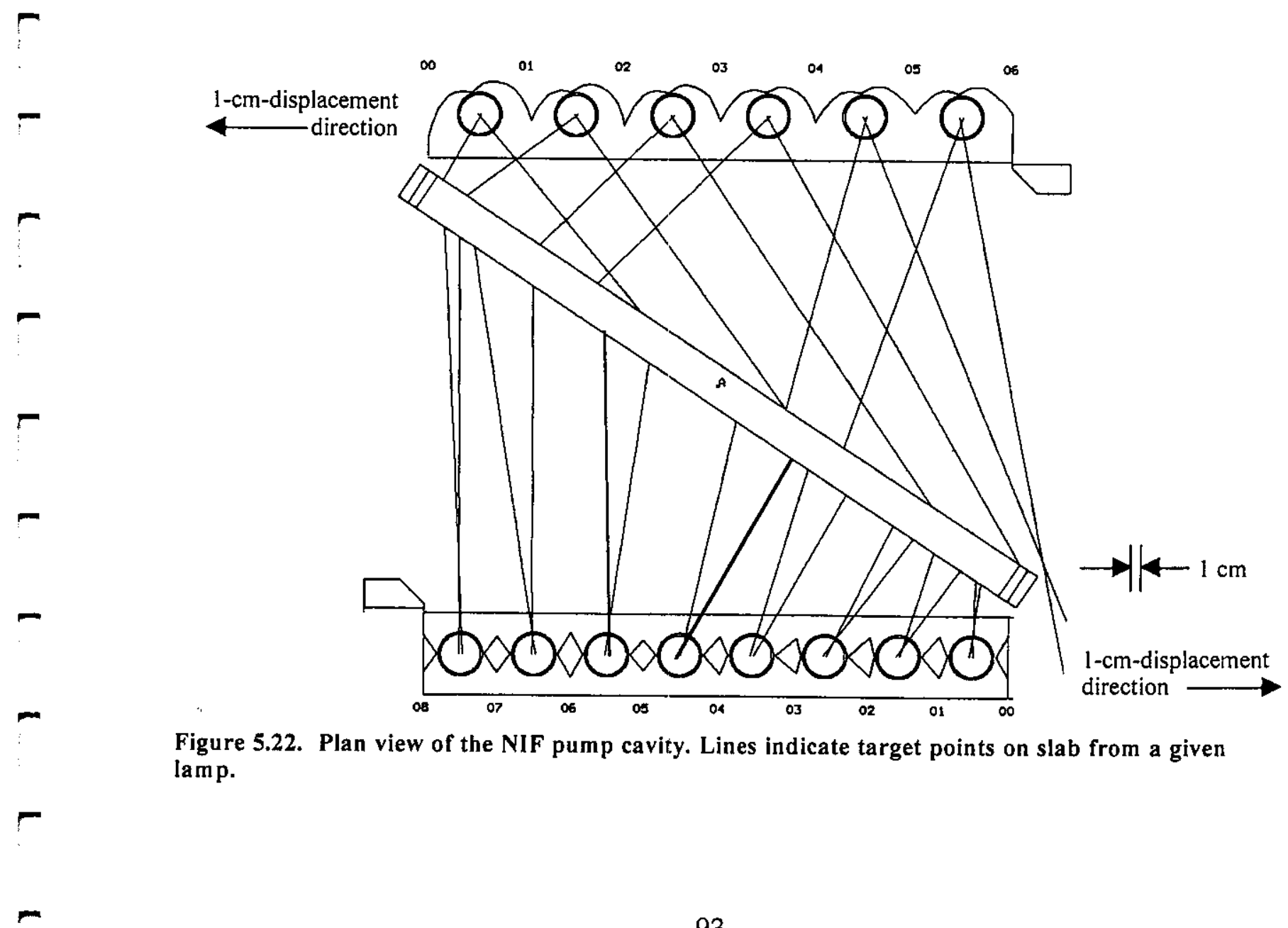

Figure 5.22. Plan view of the NIF pump cavity. Lines indicate target points on slab from a given lamp. 


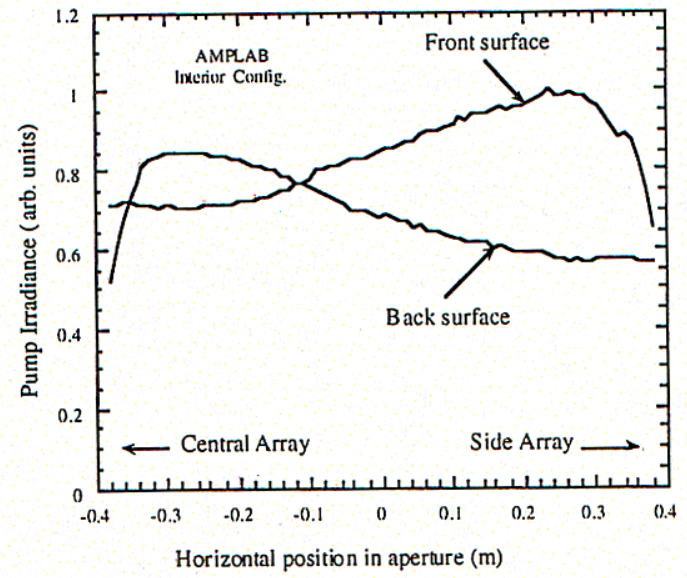

(a)

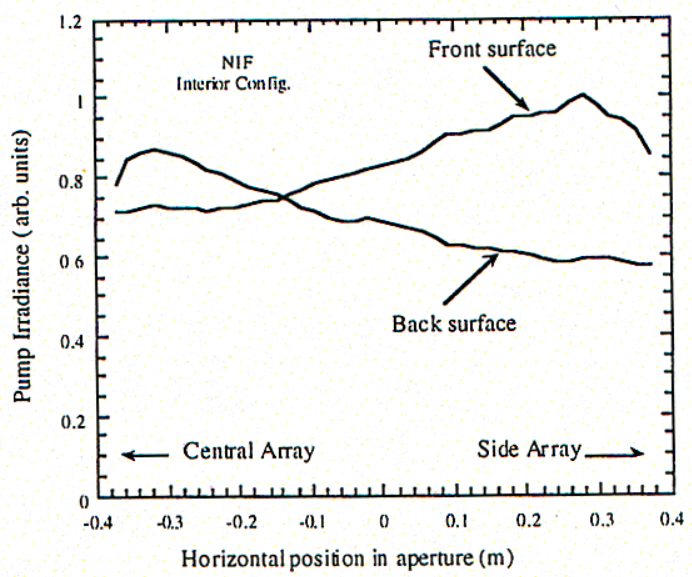

(b)

Figure 5.23. Calculated flashlamp pump-light distributions for interior slabs (a) NIF prototype amplifier tested in AMPLAB and (b) NIF baseline design.

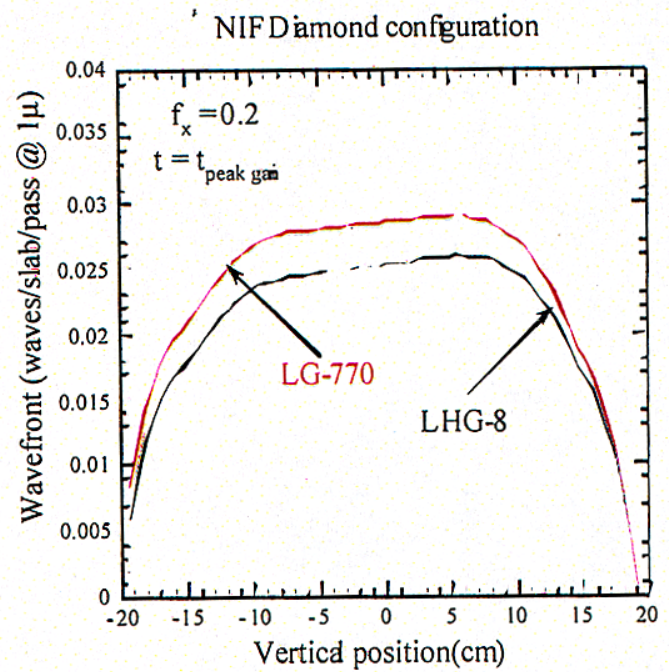

(a)

NIF interior configuration

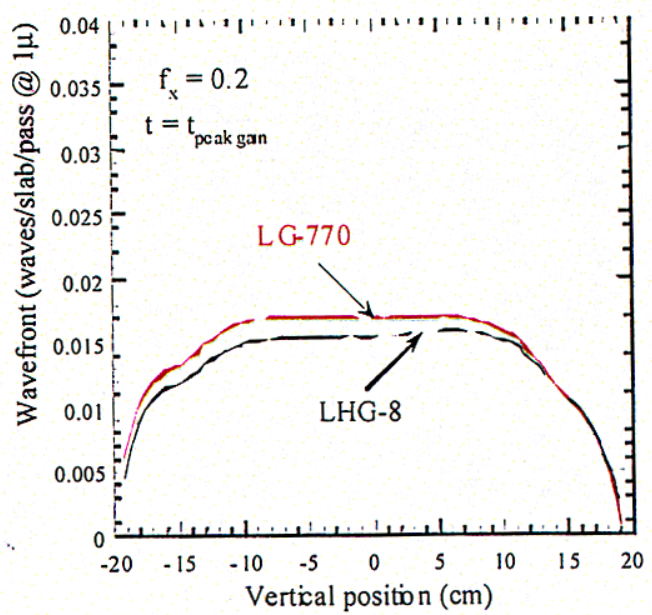

(c)

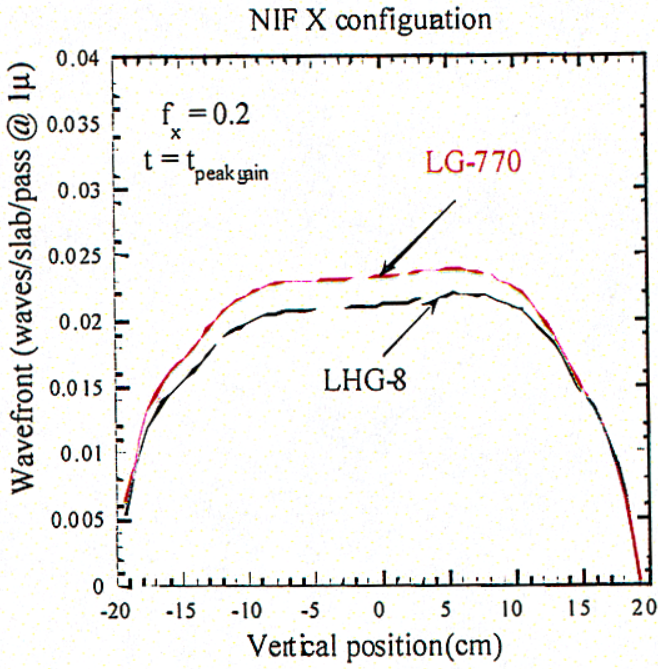

(b)

NIF system (50/50 LHG-8/LG-770)

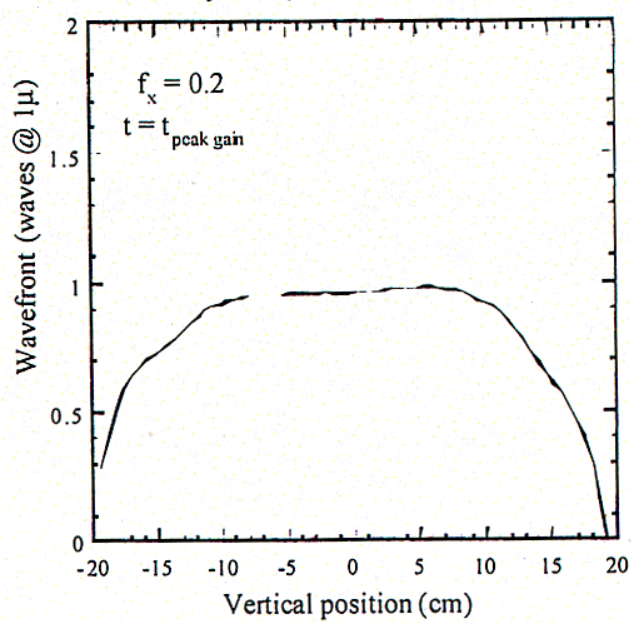

(d)

Figure 5.24. NIF wavefront predictions, vertical line-out at horizontal midplane for two laser glasses: (a) diamond configuration; (b) X configuration; (c) interior slab; and (d) NIF laser chain. 
We used PROP92 to sum the distortion of the slabs in a NIF beamline while taking into account the change in the position of the beam in the aperture from pass to pass. The results of this calculation in Figures 5.25 (a)-(d) show, respectively, a surface plot of the system wavefront distortion, the corresponding contour plot, and horizontal and vertical line-outs [5.20]. Over the $35.6 \mathrm{~cm} \times 35.6 \mathrm{~cm}$ beam area defined by the half-intensity points, the wavefront distortion is 5.5 waves $\mathrm{P}-\mathrm{V}$. We estimate the uncertainty in this prediction for NIF to be \pm 1.0 waves, or $\pm 20 \%$. This error estimate represents the sum (in quadrature) of the two most significant sources of error: the $\pm 12 \%$ uncertainty in measured wavefront distortion of interior slabs in $A M P L A B$, and an additional $\pm 15 \%$ uncertainty in the model extrapolation from AMPLAB to the NIF.
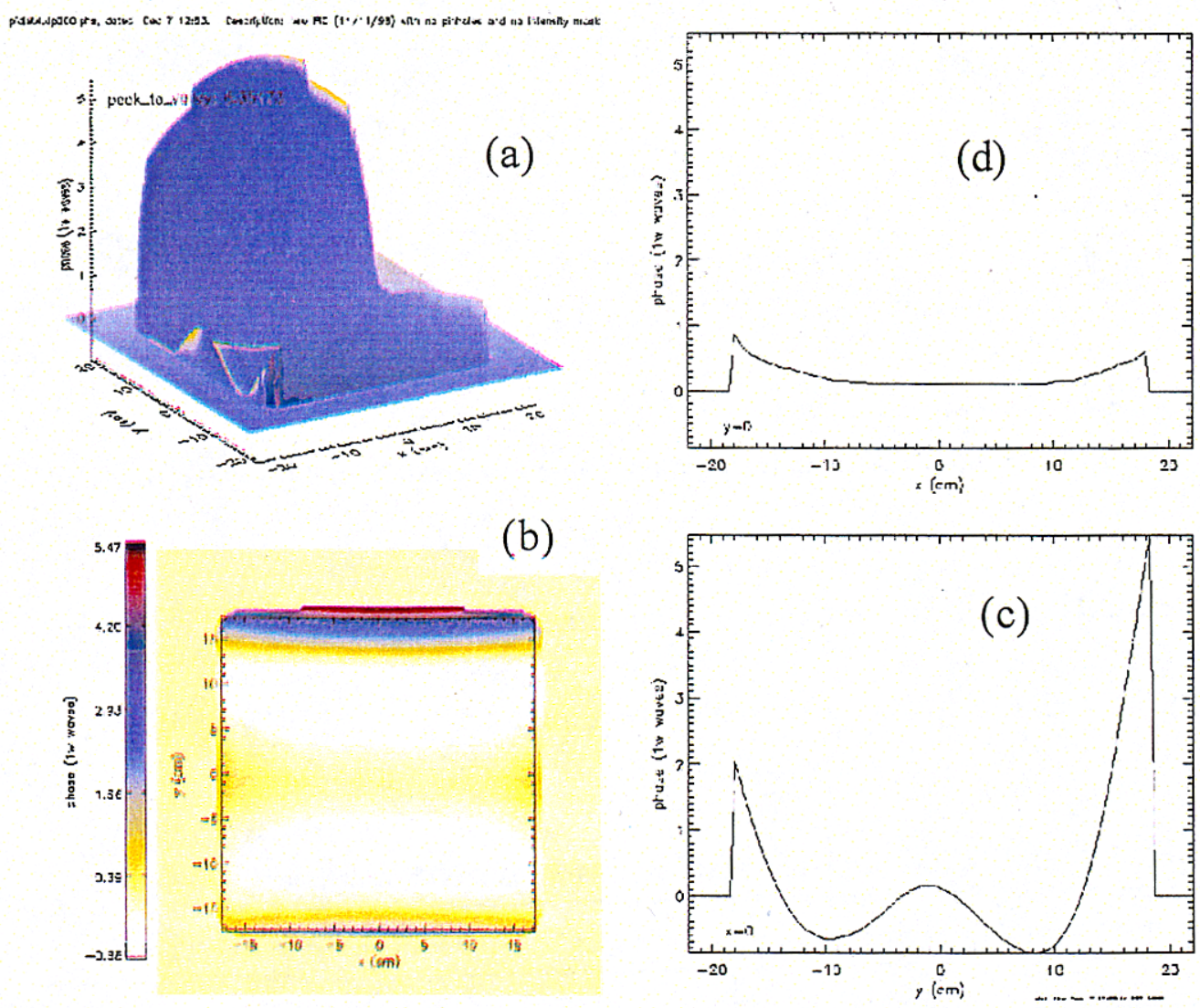

Figure 5.25. Predicted prompt wavefront distortions for the NIF beamline with $\mathbf{5 0 / 5 0}$ mix of LHG-8 and LG-770: (a) surface plot, (b) contour plot, (c) horizontal line-out at vertical midplane, and (d) vertical line-out at horizontal midplant.

The calculations presented above were for the topmost slab in a column of four. To investigate the effects of slab loading, we performed a calculation for the lowest slab in a column of four. In this case, the top surface of the slab is loaded by the weight of the other three slabs. The results of this calculation are shown in Figure 5.29 (a), which shows the static wavefront distortion contribution. The additional distortion caused by the weight of the other slabs is negligible and results in essentially a wedge of at most .006 waves. Figure 5.29 (b) shows the additional depolarization as a result of the loading, 
which was less than $.00015 \%$ at every point in the aperture. The above calculations were performed for the case of a uniformly distributed load. If the load is not uniformly distributed, i.e., there is point loading or loading over a small area, then significantly more wavefront distortion and depolarization can occur.

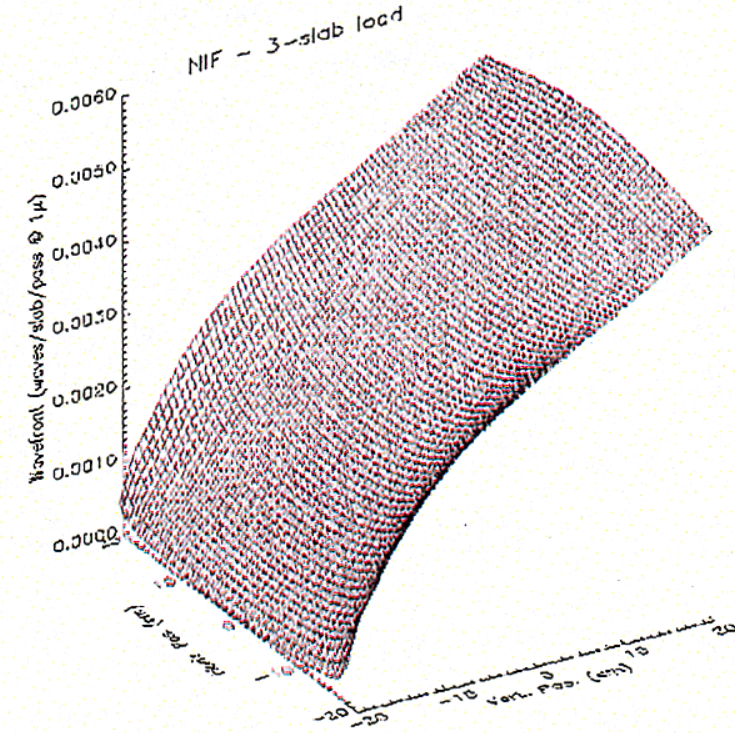

(a)

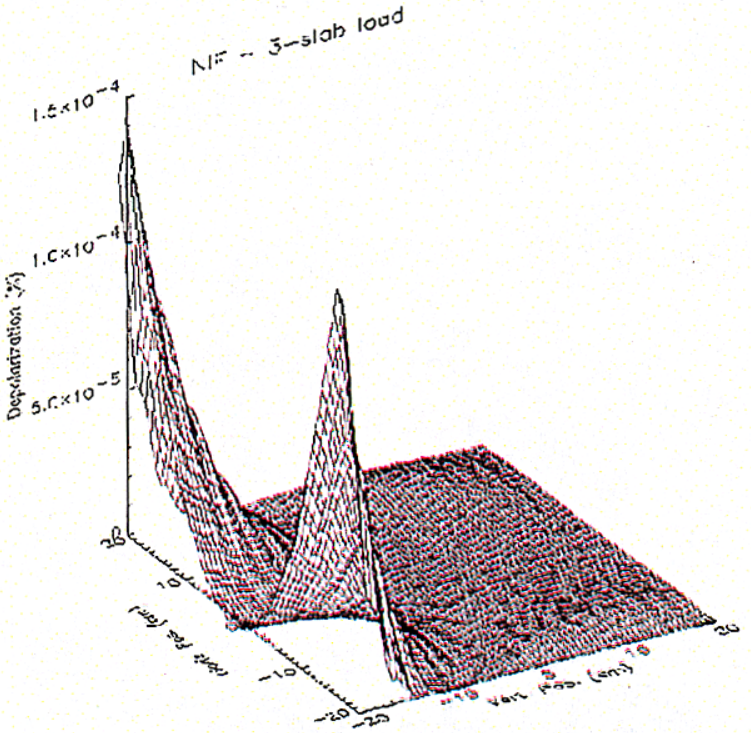

(b)

Figure 5.26 (a) and (b). Effects of a static, uniformly distributed three-slab load (a) wavefront and (b) depolarization.

\section{Compliance with NIF amplifier optical performance requirements}

As presently written, the optical performance requirements for the NIF amplifiers are as shown in Table 5.3.

Table 5.3. Optical performance requirments for the NIF amplifiers.

\begin{tabular}{|c|c|}
\hline$\Delta$ OPD across aperture - waves/slab/pass & frequency bin \\
\hline$<0.025$ & $0.0-0.5$ cycles \\
\hline$<0.05$ & $0.5-1.5$ cycles \\
\hline$<0.0125$ & $1.5-12$ cycles \\
\hline
\end{tabular}

To see if we meet the NIF requirements, we did a simple two-term Fourier decomposition of the horizontal wavefront component shown in Figure 5.23. If one writes

$$
\varphi \propto \sum_{n=1}^{2} a_{n} \sin \left\{\omega_{n} x\right\}+\sum_{n=1}^{2} b_{n} \cos \left\{\Omega_{n} x\right\}
$$

then the values for the various constants are as shown in Table 5.4. 
Table 5.4. Values for constants in Eq. (5.9).

\begin{tabular}{|c|c|c|c|c|c|c|}
\hline $\mathrm{n}$ & $\begin{array}{c}\mathrm{a}_{\mathrm{n}} \\
\text { (waves/slab } \\
/ \text { pass) }\end{array}$ & $\begin{array}{c}\mathrm{b}_{\mathrm{n}} \\
\text { (waves/slab } \\
/ \mathrm{pass})\end{array}$ & $\omega_{\mathrm{n}}$ & $\Omega_{\mathrm{n}}$ & $\begin{array}{c}\text { \# of cycles } \\
\text { across } \\
\text { aperture } \\
\text { for } \omega_{\mathrm{n}}\end{array}$ & $\begin{array}{c}\text { \# of cycles } \\
\text { across } \\
\text { aperture } \\
\text { for } \Omega_{\mathrm{n}}\end{array}$ \\
\hline 1 & .05 & .054 & .244 & .161 & 1.5 & 1.0 \\
\hline 2 & .0013 & .008 & .584 & .36 & 3.7 & 2.3 \\
\hline
\end{tabular}

We see that the $\mathrm{n}=1$ component contains frequencies that fall into the $0.5-1.5$ cycle bin. The total contribution to the wavefront from this region is .104 waves/slab/pass, roughly twice the specification. In the frequency range $1.5-12$ cycles, the total contribution to the wavefront is .0093 waves/slab/pass, within the specification of .0125 waves/slab/pass.

\subsection{Summary and conclusions for prompt pump-induced wavefront distortion}

We have presented the results of detailed analysis and modeling of the AMPLAB data. We have concentrated on the $\mathrm{C}$ aperture (LG-770 laser glass) at an explosion fraction of 0.2 , when the slab data is available. Table 5.2 above summarizes our analysis of the AMPLAB data.

We have also presented a description of our prompt pump-induced wavefront model. We have found that the model, to within a scaling factor, accurately matches the AMPLAB experimental data in all configurations and at various selected times ranging from the time of peak gain to $0.5 \mathrm{~ms}$ after peak gain. Using the model, we predict that each NIF beamline will have $5.5 \pm 1.0$ waves (P-V) of low-order wavefront distortion, with about five times greater phase variation in the horizontal direction than in the vertical direction. 


\section{7}

7 


\section{Thermal Recovery Measurements and Modeling}

Characterization of thermal recovery of the NIF amplifiers has involved detailed numerical modeling and AMPLAB experiments. All efforts were geared toward ensuring that the NIF amplifiers would achieve thermal recovery within seven hours, and have the potential for a three-hour recovery required to meet the desired increased shot-rate condition. In the sections of this chapter, the NIF thermal recovery mechanisms and requirements are reviewed, the numerical models and experiments are described, and the projection of NIF optical performance is presented.

\subsection{Thermal recovery mechanisms and requirements}

In the NIF, the principal mechanism for extracting waste heat from the laser slabs is convective cooling in the flashlamp cassettes. Gas flow at ambient temperature and pressure is used to extract the waste heat from the flashlamps and blastshields, thereby creating a cold barrier to radiatively extract the slab waste heat. The flashlamp cassette flow arrangement is shown in Figure 6.1, which depicts the down-up flow geometry. Gas injected at the tops of alternating cassettes flows down the flashlamps. After flowing across to the neighboring cassettes, the gas flows upward over a second set of flashlamps and is exhausted through the top. The NIF baseline flow rate is 20 -cfm local flow rate per lamp, as documented in Ref. 6.1. Since the gas is effectively double passed in the downup geometry, the NIF cooling requirement is $10 \mathrm{cfm}$ per actual lamp.

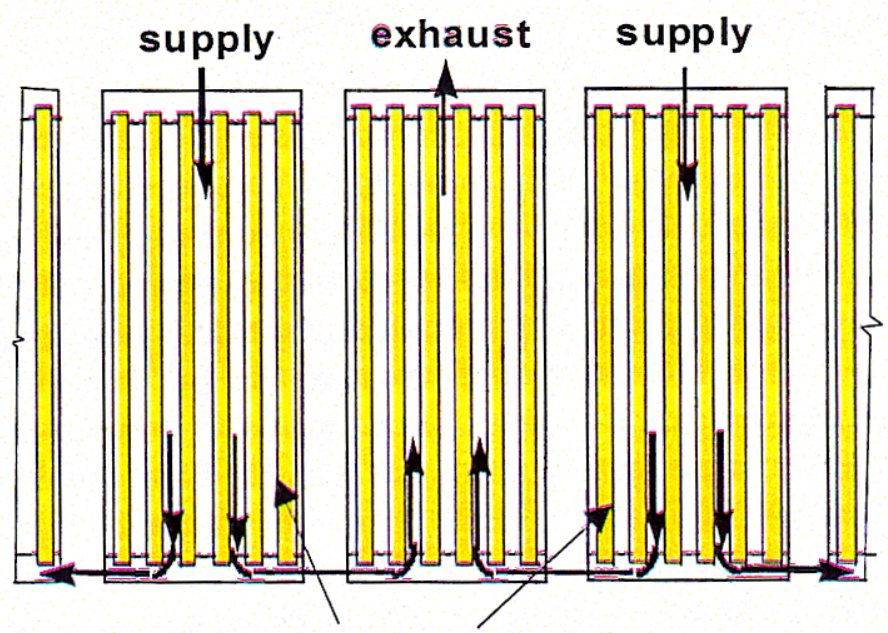

Figure 6.1. Depiction of the cooling flow arrangement in the NIF flashlamp cassettes. The supply and exhaust ports alternate, so that the gas is "double-passed" through the cassettes.

Historically we have grouped optical distortions into two categories: (1) distortions in the laser slabs due to temperature gradients, and (2) distortions in the gas columns within and neighboring the amplifier that are due to temperature-difference-driven convection currents. The recovery requirements for each category are as follows: 
Gas column

distortions:

The NIF design requirement is that the added beam

divergence due to thermal-recovery-driven convection currents shall not exceed $5 \mu \mathrm{rad}$ at the end of the recovery period.

Slab distortions: The thermal-gradient-driven optical distortion in the slabs shall not exceed 0.04 waves/slab/pass $(\mathrm{P}-\mathrm{V})$ at the end of the recovery period. For this coherently additive distortion, this translates to 2.2 waves for the 54 "effective" slabs in the multipass NIF architecture.

For the NIF baseline shot rate of one shot every eight hours, the requirement is that the above optical distortion limits be achieved within seven hours after the start of recovery. For the accelerated shot rate of one shot every four hours, the thermal recovery constraint is reduced to three hours. Both time limits are predicated on allowing one hour for final alignment where the cooling system is turned off to eliminate flow-induced vibration disturbances.

\subsection{Numerical models}

Two thermal models have been developed to characterize the thermal and optical behavior of the AMPLAB prototype amplifier module and the NIF amplifiers. Below is a detailed description of each of the model sets.

\section{Lumped-mass thermal model}

The lumped mass model treats the amplifier as a set of discrete entities, as described in Figure 6.2. The main entities are laser slabs, central cassette blastshield, side cassette blastshield, central cassette flashlamps, side cassette flashlamps, and flashlamp cassette reflectors. Radiative heat transfer is the only allowed mechanism for removing the slab waste heat. This model, which is described in Ref. 6.1, treats each of the entities as a lumped mass, with analytically prescribed radiation exchange factors and analytically prescribed forced convection heat transfer coefficients dictating the heat transfer processes in the flashlamp cassettes. The purpose of this model is to describe the overall global temperature behavior of the system, primarily as a check on the detailed threedimensional model described in the next section. Since the entities represent lumped masses, this model is incapable of characterizing the temperature distribution within an entity. A characterization of the energy exchange processes incorporated into this model is given in Figure 6.3. In instances where the slab is adjacent to a "downstream" flashlamp cassette, upstream cassette effects are included by incorporating the thermal mass and energy of the lamps, blastshields, and reflectors of the upstream cassette. In this manner, we account for the heating of the gas in an upstream cassette prior to reaching the cassette of interest. 


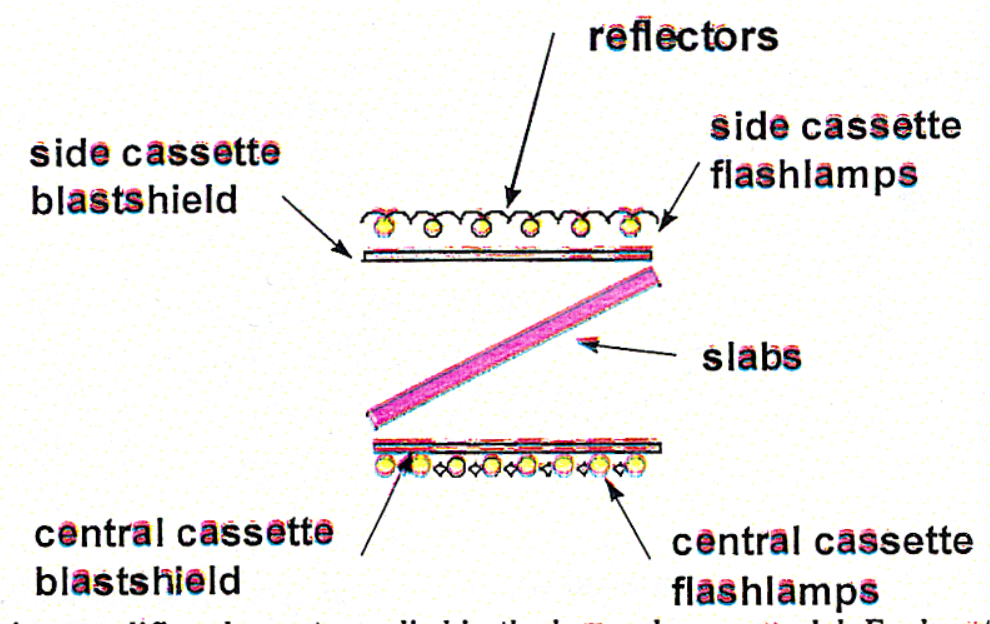

Figure 6.2. The major amplifier elements applied in the lumped-mass model. Each entity represents the lumping of the masses of all amplifier elements in that category.

\section{Three-dimensional thermo/mechanical/optical Model}

This model actually consists of a sequence of four (4) computer codes $[6.2,6.3,6.4$, and 6.5]. This model includes details of the amplifier geometry, including reflector shapes, discrete flashlamps, slab holder frame, and the slab masks. Additionally, details of the slab, including the edge cladding, are incorporated into the model.

The first step in the calculation sequence is the evaluation of the radiation exchange factors, using a Monte-Carlo algorithm [6.2]. The finite-element mesh generated for the heat transfer calculation is used in this calculation. Then the temperature distribution in the system for the entire recovery cycle duration is calculated, using a finite-element heat transfer computer program [6.3]. The heat transfer calculation starts with specified temperatures, based at this time on experimental data. The temperature distribution is then used as input to a finite-element mechanics calculation to evaluate deformation and stress in the laser slabs [6.4]. The final step in the calculation sequence is to use the calculated temperature, deformation, and stress distributions in a ray-trace algorithm to evaluate the optical path length (OPL) variations across the aperture [6.5]. The effects of $\mathrm{dn} / \mathrm{dT}$, displacement, and stress are included in the OPL evaluation.

As noted above, in this model sequence the radiation exchange factors are evaluated in detail. Because this model does not contain details of the fluid transport processes in the flashlamp cassettes, analytically derived heat transfer coefficients are prescribed for each surface in contact with the flashlamp cooling fluid. This model provides details of the temperature, displacement and stress distributions within the slab as well as temperature distributions on all other entities in the system. The only constraints on spatial fidelity are memory limitations placed by the computer platforms. Additional details of this model are provided in Ref. 6.6. 


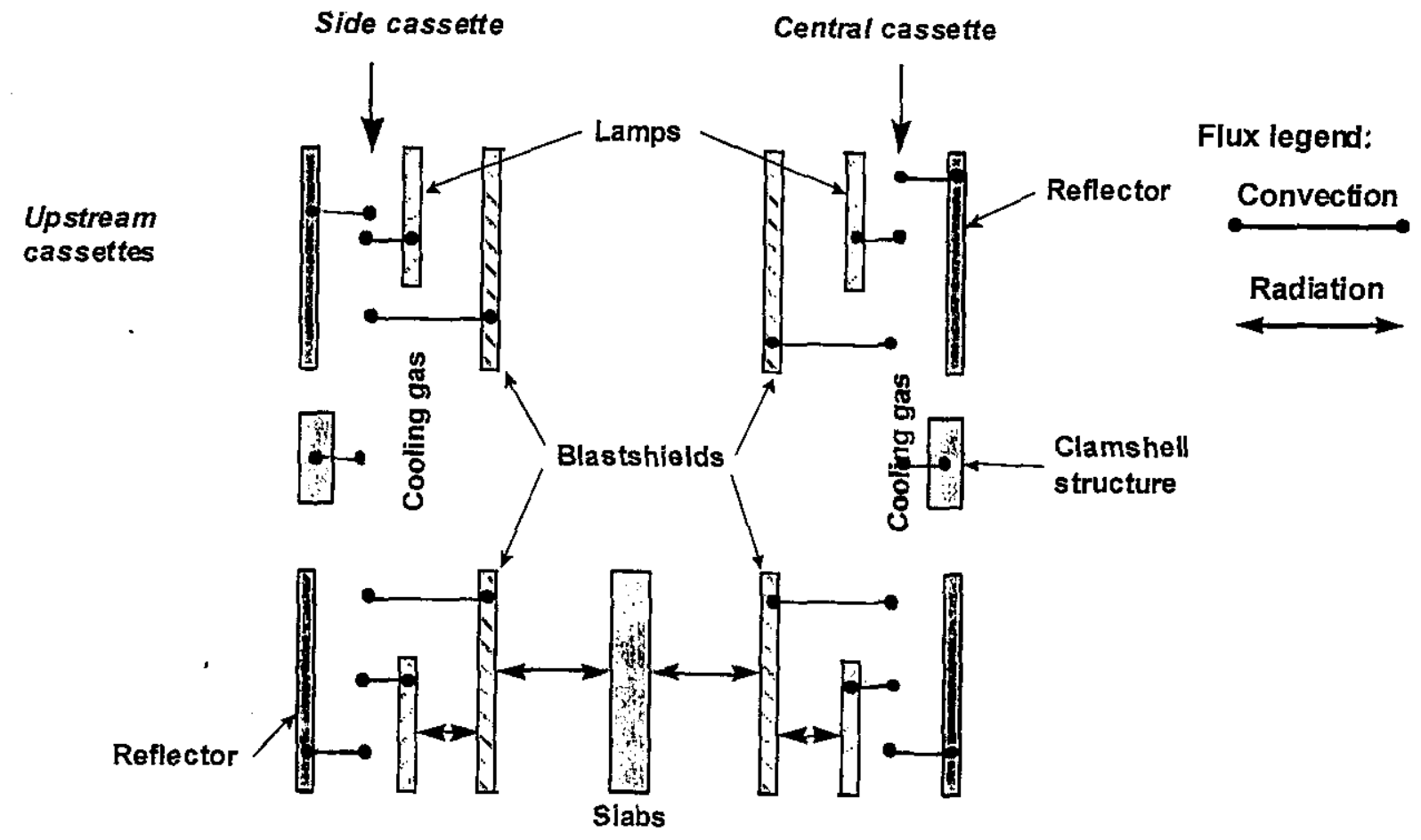

Figure 6.3. An entity and energy flow diagram for the lumped mass model. Note that the effect of the upstream elements on the cooling fluid is included in detail.

\section{Model geometry variations}

There are a number of geometry and optical element differences between NIF and AMPLAB that required incorporation into the models. These are depicted in Figure 6.4. In AMPLAB the thermal cassette was a combination of 34-mm- and 40-mmthick slabs, whereas for both the optical side of AMPLAB and the NIF, the slab thickness was $40 \mathrm{~mm}$. Additionally, the side cassette reflector on the thermal side of $A M P L A B$ was flat, while all other side cassette reflectors (both AMPLAB and NIF) used the "involute" shape.

In AMPLAB, the optical and thermal measurements were made in a 2-slab-long configuration. Thus, all slabs in this case are end slabs. That is, they are all exposed to the beamtubes. In the NIF, however, the main amplifier has nine interior slabs, and the power amplifer has three interior slabs. To accommodate this geometry variation in the model, as shown in Figure $6.4 \mathrm{~b}$, radiation surface properties were varied. For interior slabs, both end planes were treated as symmetry boundaries (perfect reflectors). For end slab conditions, one end plane was treated as a "black" surface to simulate the effect of the adjacent long beamtube. 


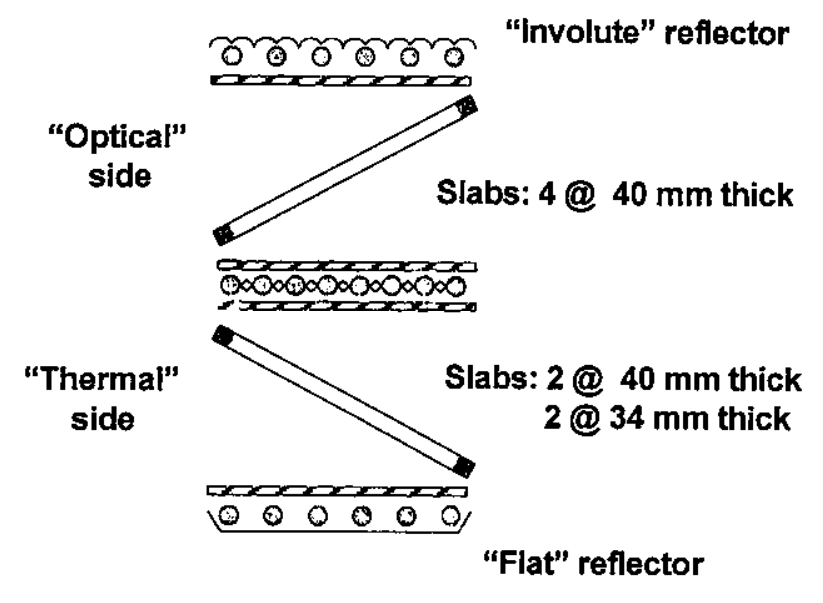

(a)

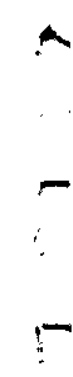

Interior slabs: Mirror (symmetry) plane

(b)

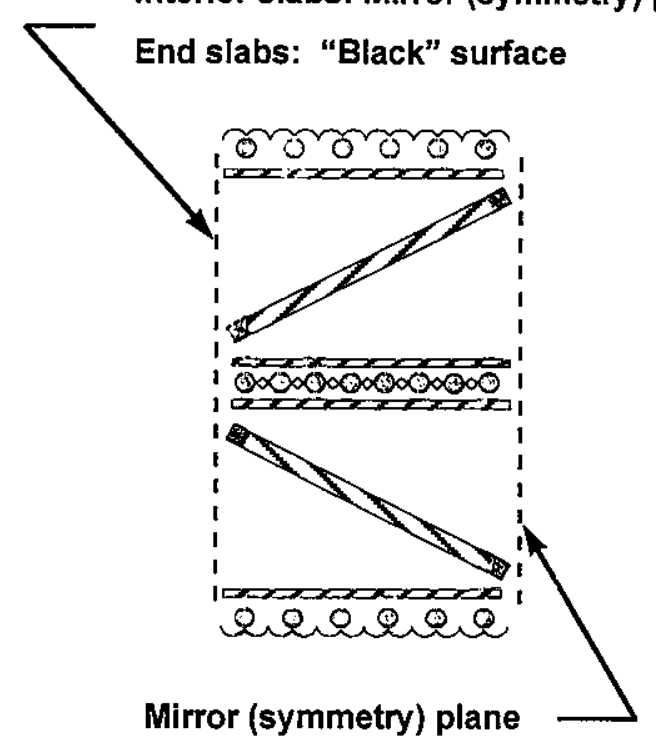

Figure 6.4. Depiction of geometry and boundary condition differences used in the model calculations. (a) geometry differences between "optical" and "thermal" sides of AMPLAB and (b) boundary conditions applied to the end planes to simulate interior and end slabs of the amplifier.

\subsection{AMPLAB temperature measurements}

A key aspect in characterizing NIF amplifier thermal recovery was the instrumentation of AMPLAB to measure temperatures in the amplifier cavity. AMPLAB is a facility designed to test the optical and thermal performance of the NIF amplifiers. It consists of a NIF-like, two-slab-wide configuration, but is only two slabs long in the optical propagation direction. The four slabs in each cassette are labeled A, B, C, and D from the top. In one slab cassette, in what is termed the thermal measurement side (see Figure 6.5a) three slabs were instrumented with temperature measuring devices $(\mathrm{A}, \mathrm{C}$, and $\mathrm{D}$, as shown in Figure $6.5 \mathrm{~b}$ ). The associated side cassette blastshield and a side cassette flashlamp were similarly instrumented. 
(a)

(b)

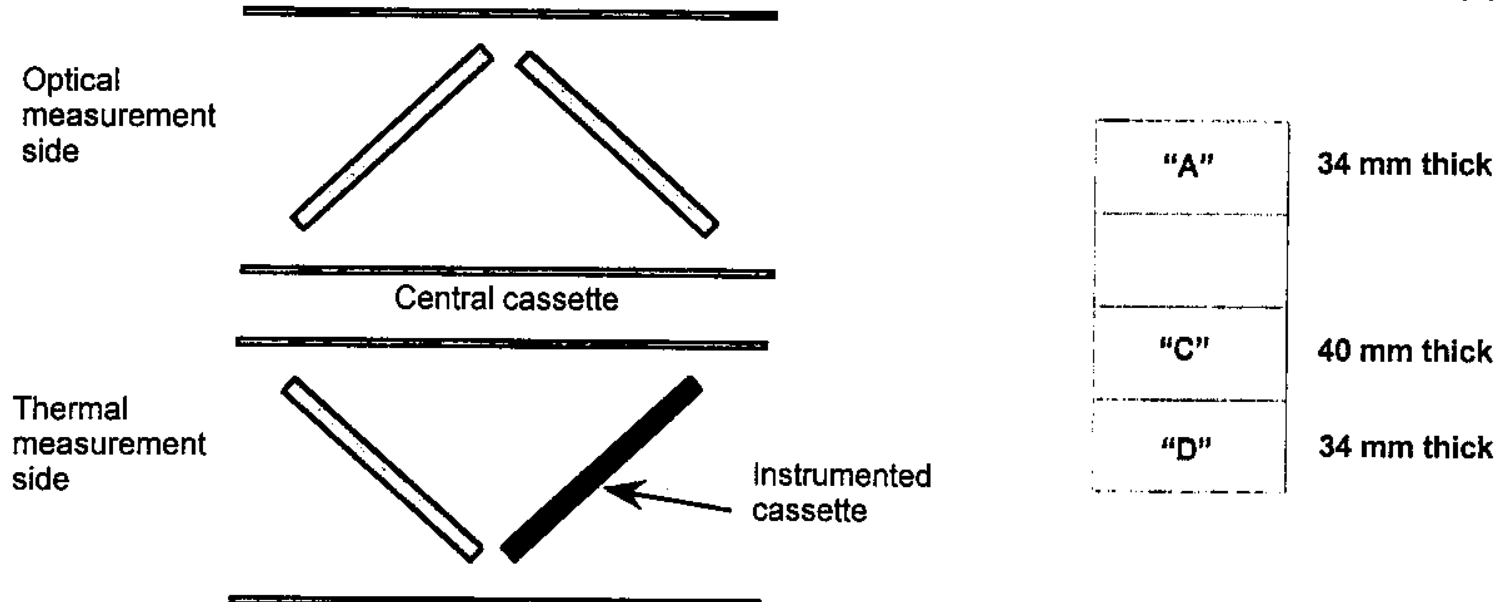

Figure 6.5. Schematics of the instrumented AMPLAB slab cassette. One slab unit was instrumented with thermocouples to measure temperatures in the slabs during the thermal recovery cycle. (a) plan-view schematic of the AMPLAB experimental configuration. (b) slab position nomenclature, and slab thickness in the instrumented cassette.

A total of 59 type-E thermocouples and one fiber-optic probe were placed in the slabs. Typical locations of the thermocouples and fiber probe are shown in Figure 6.6a. The thermocouples were placed in $1-\mathrm{mm}$ diameter holes drilled through the slab, with the junction at the mid-thickness of the slab (see Figure 6.6b). The fiber probe was placed in a blind hole drilled halfway through the slab. Four thermocouples were in contact with the flashlamp side of the blastshield, and four thermocouples and one fiber probe were in contact with one side cassette flashlamp. Thermocouples were selected (over thermisters) since they do not provide an added heat source that could alter the temperature reading in the low thermal conductivity glass slabs. It was estimated that the thermocouple uncertainty was less than $\pm 0.05^{\circ} \mathrm{C}$. This instrumented cassette contained a combination of 34-mm-thick and $40-\mathrm{mm}$-thick slabs. All other slabs in the facility were $40 \mathrm{~mm}$ thick, which is the NIF thickness specification.

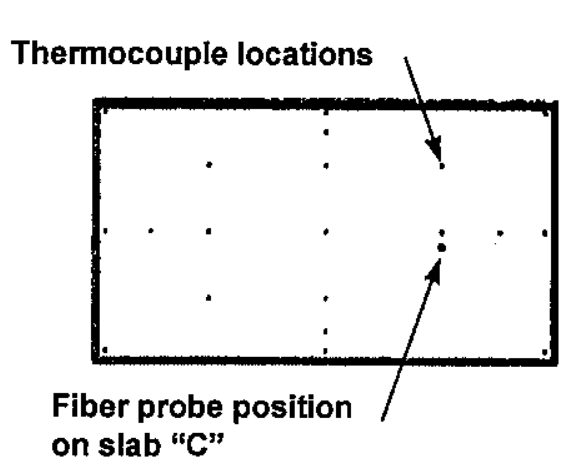

(a)

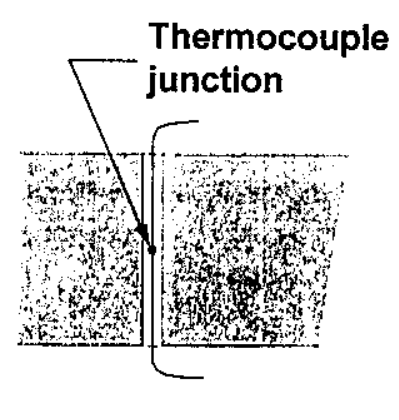

(b)

Figure 6.6. Details of thermocouple placement in the slabs of the instrumented cassette. (a) Typical locations of the thermocouples in slabs " $A$ ", "B", and " $C$ ", and (b) depiction of the thermocouple location in the through-hole. 
Because of high electrical currents in the flashlamps, the thermocouples were disconnected from the data acquisition system during the flashlamp firing. The thermocouples were reconnected from 3 to 5 minutes after the flashlamp firing, which allowed sufficient time to assess the integrity of the lamps. This also corresponded to the time when the flashlamp cooling system was turned on. This delay in activation of the cooling system is similar to that projected for the NIF.

The starting temperatures for the thermal recovery model calculations were taken directly from AMPLAB measurements. Figure 6.7 shows fiber probe measurements of the slab temperature for three successive firings of the flashlamps. For these shots, the amplifier was in the diamond configuration. With each firing of the lamps, the probe tip was preferentially heated. This over-driving of the probe temperature required about six minutes to decay out. The approach for extrapolating back to shot time to correct for the prompt probe heating is shown in Figure 6.7. In this instance, the slab temperature rise was nominally $0.65^{\circ} \mathrm{C}$. A similar approach was used to obtain starting temperatures for the flashlamps and blastshields. In the case of the flashlamps, however, this projection approach is less accurate because of the large radiative losses that occur due to the high lamp temperature.

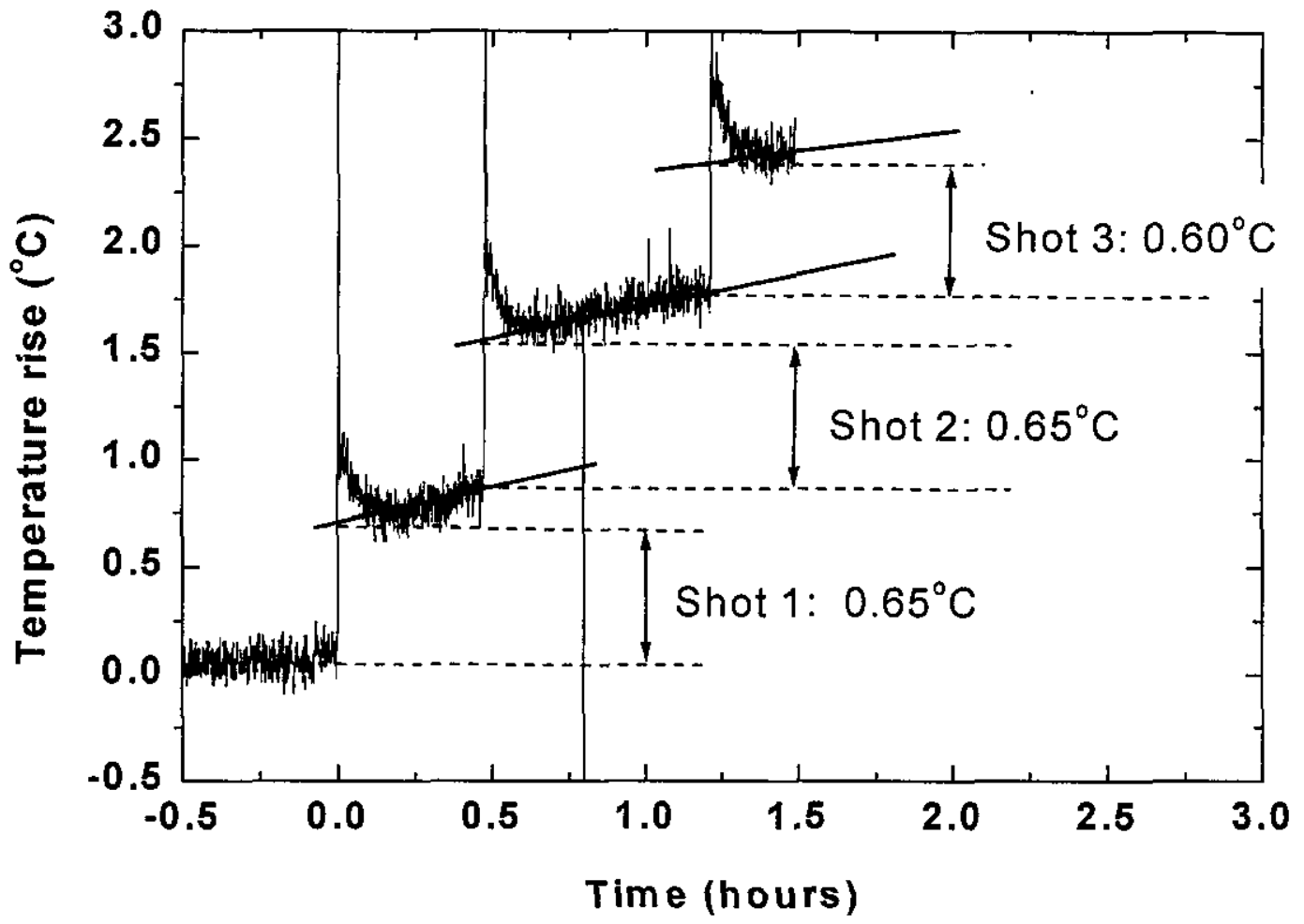

Figure 6.7. Slab temperature rise as measured by the fiber probe, over three successive shots. Also shown is the projection of the temperature back to shot time to correct for the prompt heating of the probe. Flashlamp cooling was absent in these tests. The amplifier was in the diamond configuration.

Table 6.1 summarizes the starting temperature conditions applied in both the lumpedmass model and the three-dimensional model, where flashlamp, edge cladding, blastshield, and slab temperatures are given for AMPLAB slabs and NIF end and interior slabs. Several key features are noted. The first feature is the starting temperature 
differences for the AMPLAB 34-mm- and 40-mm-thick slabs. It was determined that simple thickness scaling was appropriate since the slabs are optically thick to the flashlamp light. That is to say, the temperature difference between the 34-mm- and 40mm-thick slabs was $15 \%(40 \mathrm{~mm} / 34 \mathrm{~mm})$. Additionally, the interior slab temperature was $11 \%$ greater than the end slabs due to pumping differences. Finally, the flashlamps in the AMPLAB thermal side cassette were judged to be $4^{\circ} \mathrm{C}$ hotter than the flashlamps in the optical side cassette because of differences in the reflector designs.

Table 6.1. Initial temperature conditions used in the AMPLAB and NIF thermal recovery calculations.

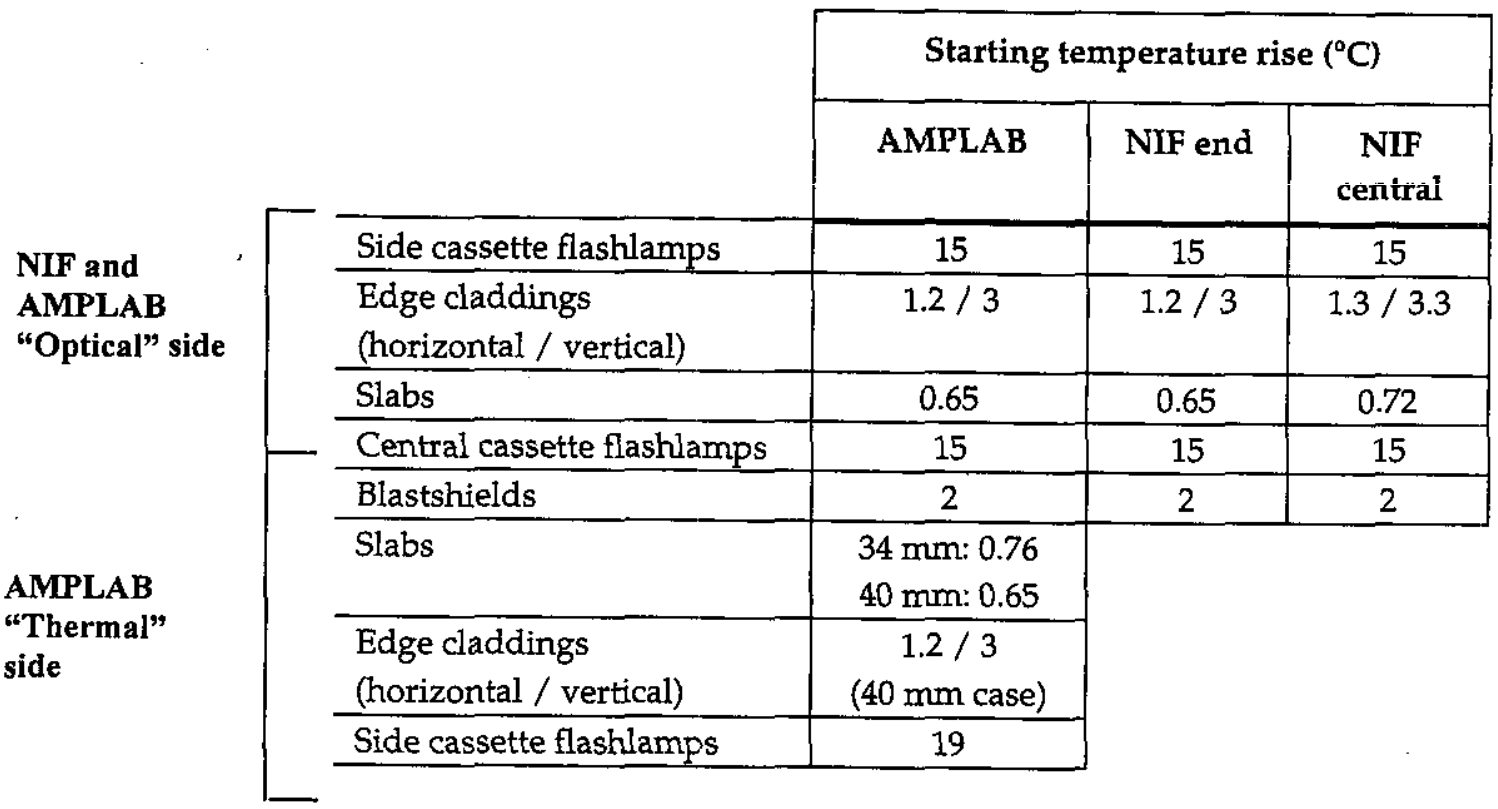

Figure 6.8 gives representative temperature distributions across the " $\mathrm{A}$ " and " $\mathrm{D}$ " apertures for a shot at $20 \%$ flashlamp explosion fraction followed by forced gas cooling of the flashlamps with ambient-temperature air. Several key observations are:

1. Recovery is clearly evident through the reduction in temperature during the first eight hours after the shot.

2. Strong coupling between the edge cladding and the slab masks is evidenced by the rapid reduction in temperatures at the edges of the slab. Note that the initial edge-cladding average temperature rise on the vertical edge cladding elements was roughly $3^{\circ} \mathrm{C}$.

3. An asymmetry is evident in both slabs, but more prominently in slab " $D$ ". In both figures, the right edge of the aperture is nearest the central cassette, which was more aggressively cooled than the side cassette. This likely accounts for the asymmetry.

4. The residual temperature after 10 hours is indicative of a slightly warmer than ambient flashlamp cooling gas. In this particular experiment, a chiller was used to establish the cooling gas temperature, thus providing the potential for a slight temperature offset. 


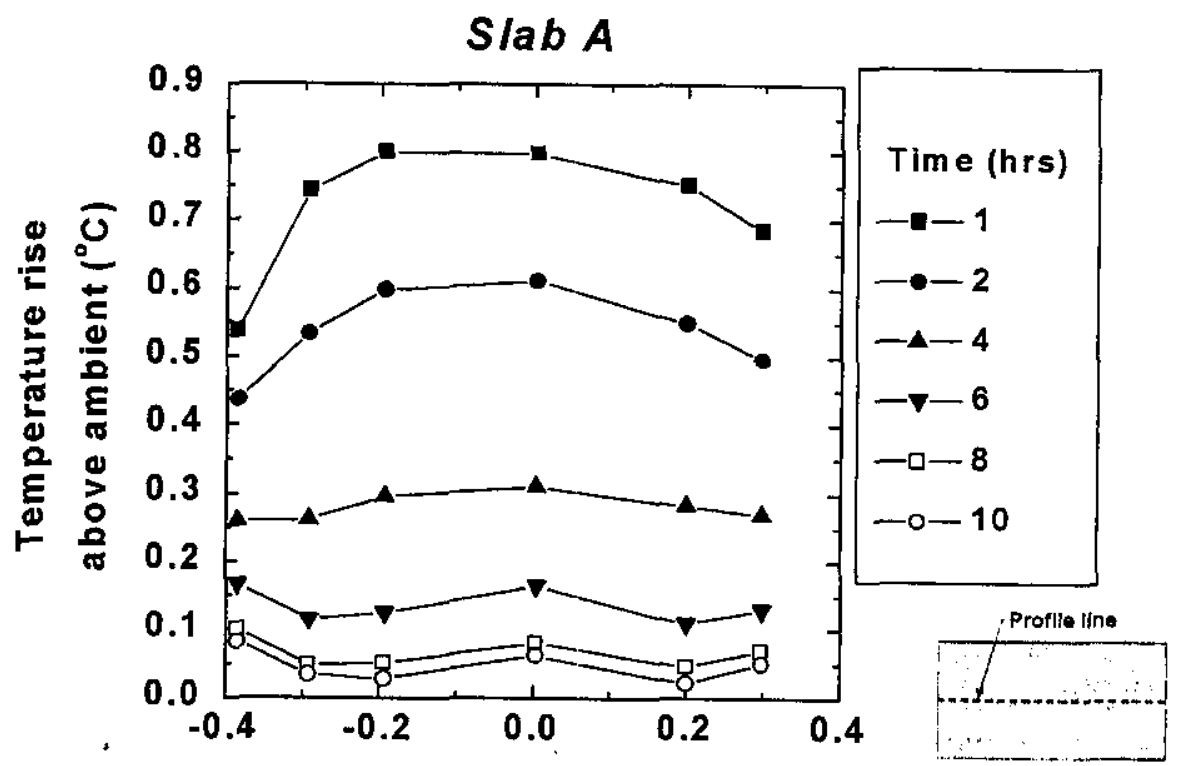

Distance from slab centerline (m)

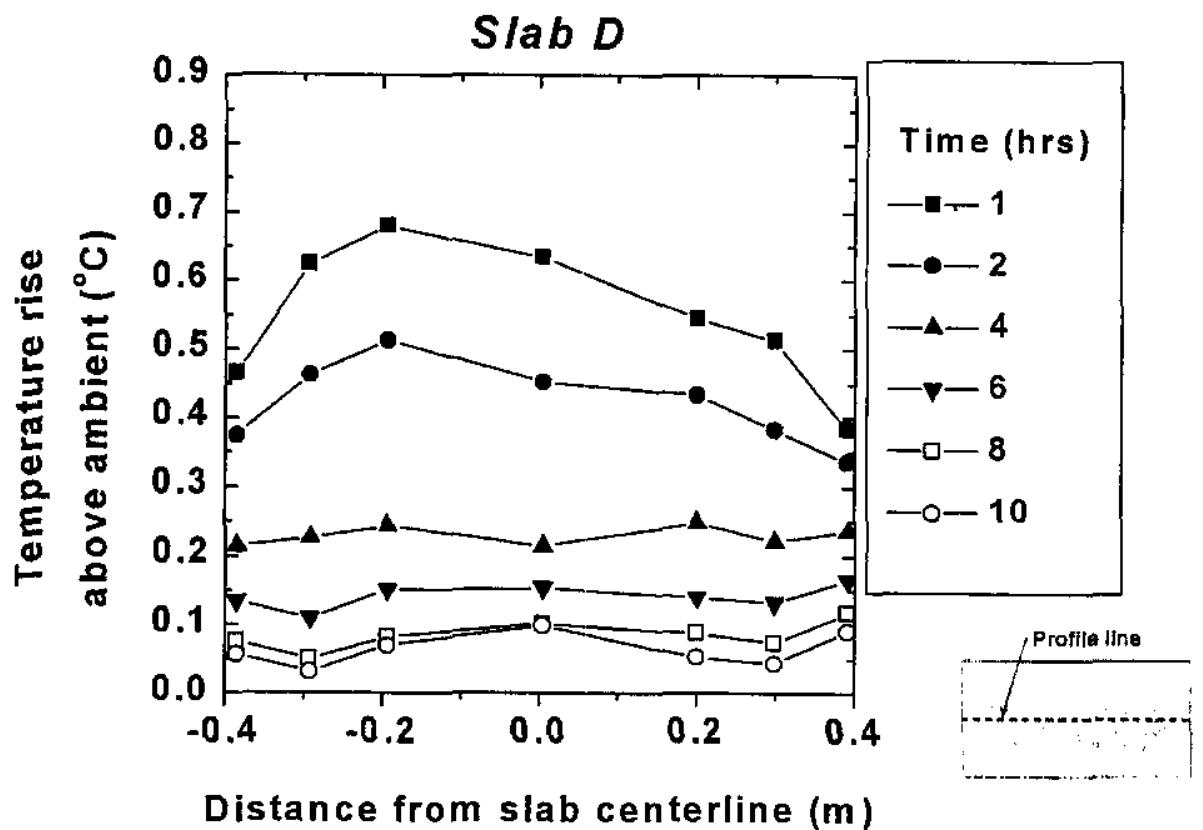

Figure 6.8. Experimentally measured temperature profiles across the long dimension of the laser slab. All times are measured from shot time. Note that both slabs were $34 \mathrm{~mm}$ thick, and the amplifier was in the diamond configuration. (a) Slab A temperature distributions and (b) slab D temperature distributions.

\subsection{Comparison of AMPLAB temperature measurements with model predictions}

Since the flashlamps are the hottest element in the amplifier, and thus represent the dominant source of residual heat that could be transported to the laser slabs, it is important that the convection coefficient values applied to the flashlamps be correct. In 
the AMPLAB tests, the temperature of a side cassette flashlamp was measured using both thermocouples and a fiber probe. Figure 6.9 gives the comparison of model predictions to the measured temperatures. There is excellent agreement between the two experimental measurements, thus giving high confidence in the experimental values. Because the thermocouples were disconnected during the flashlamp firing, the first six minutes of thermocouple data were lost in this case. Also, because the fiber probe was exposed to flashlamp light, it was overdriven and required several minutes to equilibrate with the slab, again resulting in the loss of early time data. Because of this early-time loss of data, the starting temperature for the flashlamps used in the calculation was obtained from uncooled data using the projection approach discussed earlier (see Figure 6.7). Returning once again to Figure 6.9 , there is reasonably good agreement between the measured temperature and the model prediction. At times less than 0.6 hours, there is at most a $20 \%$ difference, with the model results being conservative. These differences are likely due to slight discrepancies in the heat transfer coefficient as well as to inaccuracies in upstream component effects on the cooling fluid. However, this generally good agreement gives us confidence in the cooling parameters associated with the cooling gas flow.

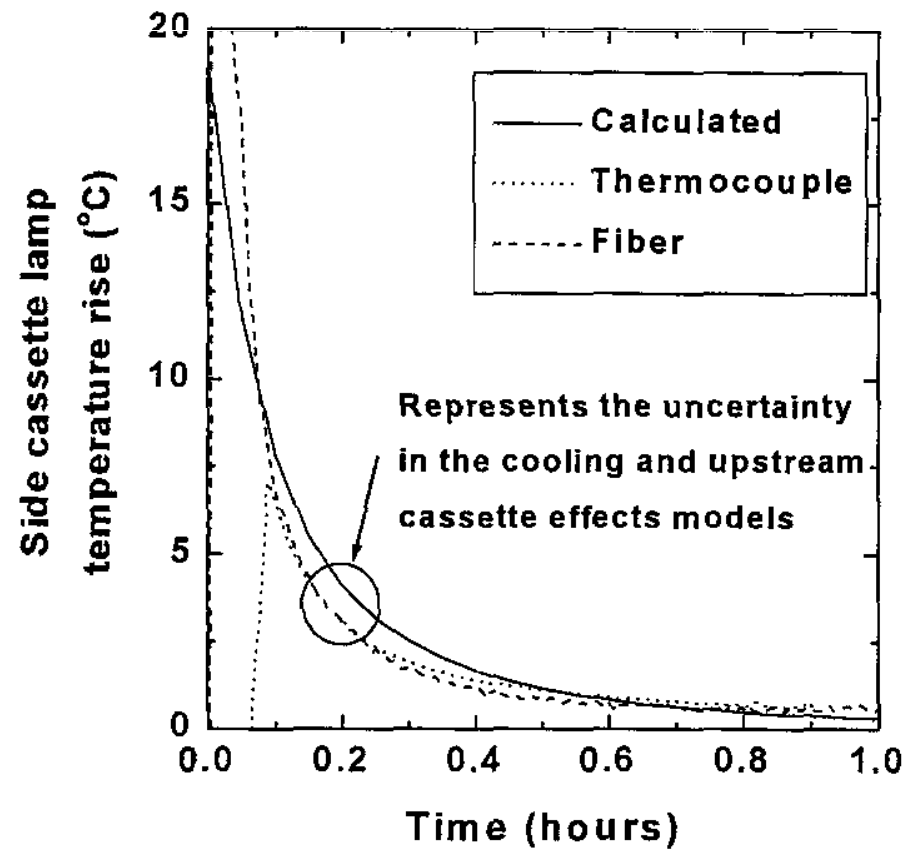

Figure 6.9. Comparison of the calculated and measured flashlamp temperature for an AMPLAB ambient cooled recovery case. Measured values using both thermocouples and the fiber probe are shown.

Figs. 6.10 and 6.11 address the issue of thermal contact at the slab edges. This is particularly important since the measured edge cladding average starting temperature was as large as $3^{\circ} \mathrm{C}$, while the neighboring slab temperature is only roughly $0.65^{\circ} \mathrm{C}$. This edge effect was evidenced in the data of Figure 6.8. Without proper removal of this waste heat from the edge cladding, this temperature difference can greatly contribute to the overall slab distortions. Figure $6.10 \mathrm{a}$ depicts the geometry near the edges of the slab, where the edge cladding is thermally coupled to the cassette frame and mask structure by 
conduction through thin air gaps. Figure $6.10 \mathrm{~b}$ shows the initial temperature distribution in the edge cladding and slab due to pumping and ASE absorption, calculated using the absorption coefficient for $1.05-\mu \mathrm{m}$ radiation. This geometry and initial temperature condition were applied in two-dimensional heat transfer [6.7] simulations to determine the appropriate value for the air-filled gap thermal resistance. Results are presented in Figure 6.11, where the experimentally measured edge cladding temperature is compared to numerical predictions for two values of the air gap resistance. These results indicate that a gap conductance of $30 \mathrm{~W} / \mathrm{m}^{2}-\mathrm{K}$, which corresponds to a 1-mm-thick air gap, most closely matches the experimental data. The higher conductance value corresponds to a $0.33-\mathrm{mm}$ thick air gap, and is clearly incorrect. It is important to note that from examination of the assembly drawings of the thermal cassette components, the gap would be projected to be from 0.5 to $1 \mathrm{~mm}$ thick, which is consistent with the thermal results.

(a)
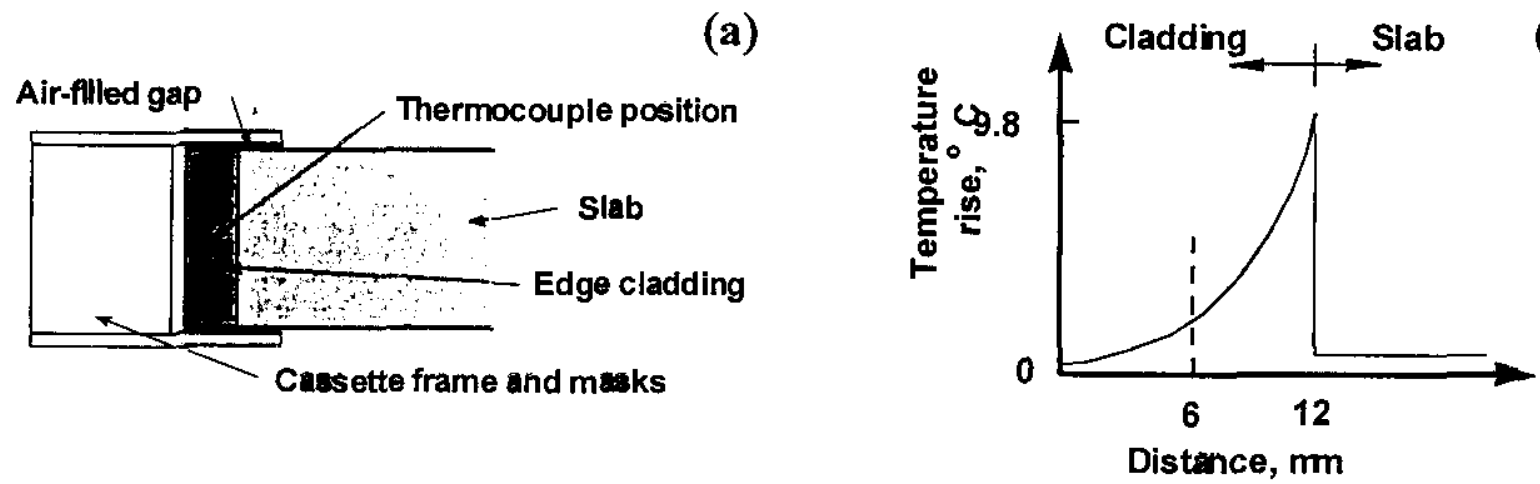

(b)

Figure 6.10. The geometry and edge cladding temperature profile used in the calculations to quantify the air-filled gap thermal impedance.

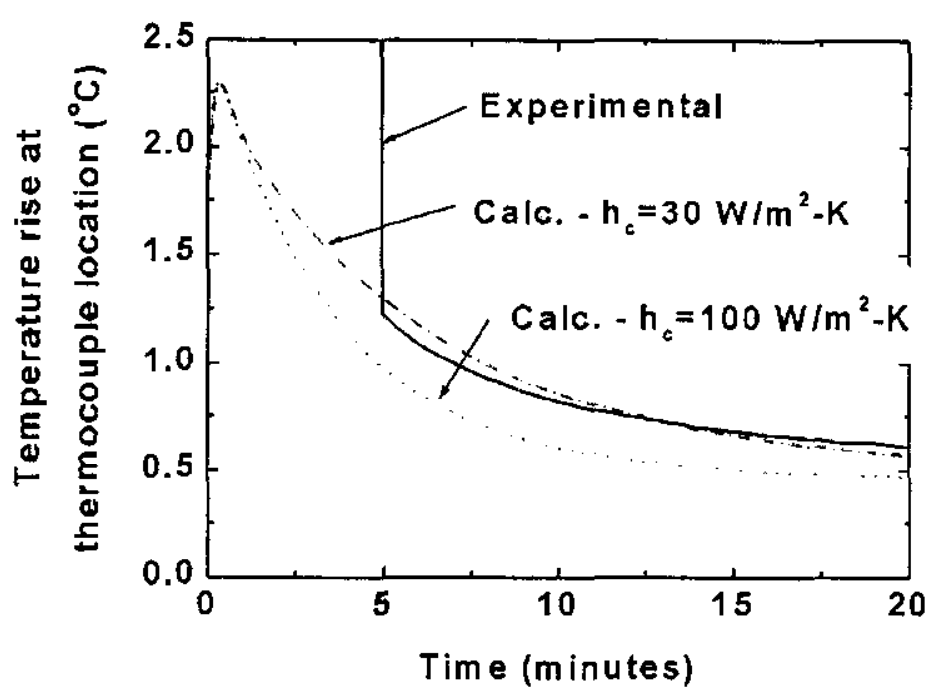

Figure 6.11. Comparison of calculated and measured temperature at the mid-point of the side edge cladding. 
Part of the purpose of developing two numerical models was for model validation purposes. Figure 6.12 compares predicted average slab temperatures for both interior and end slabs using the lumped-mass model and the full three-dimensional thermal model. In this calculation set the starting slab temperature was specified as $0.65^{\circ} \mathrm{C}$ above ambient for both slab types. Clearly there is excellent agreement between the two vastly different models. This gives us good confidence in the simple algebraic relationships that were used to specify the radiation exchange factors in the lumped-mass model, as well as the confidence in the Monte-Carlo calculations used to develop the exchange factors for the 3-D thermal model.

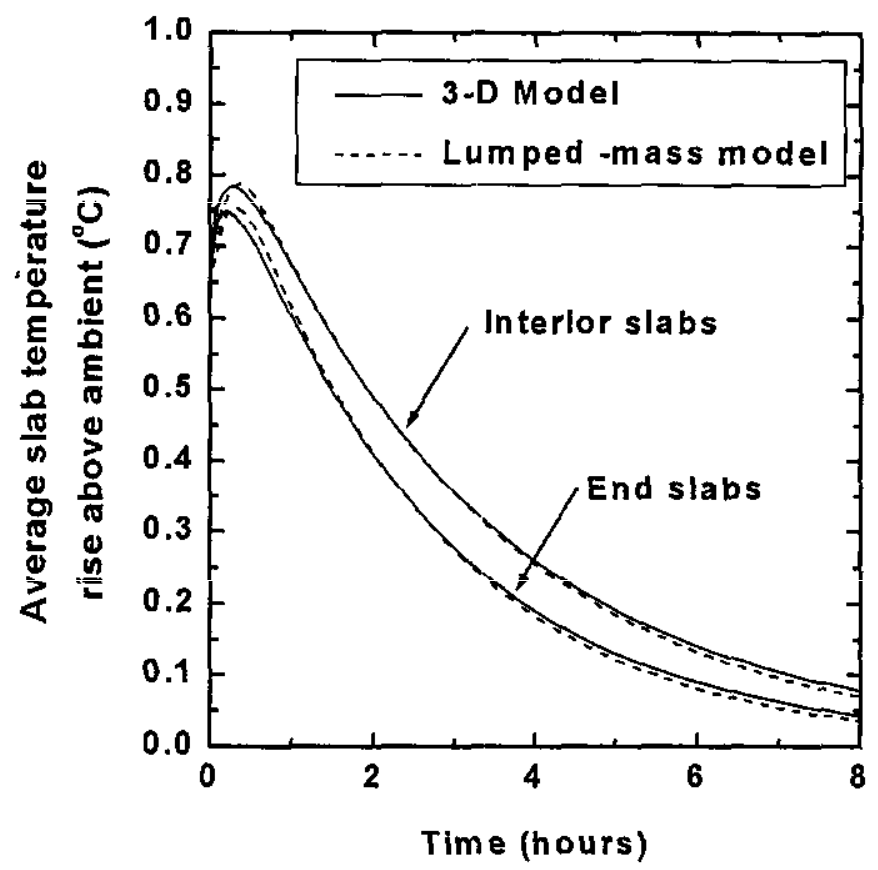

Figure 6.12. Comparison of slab average temperature obtained using the two numerical models. Both interior and end slab conditions are given.

Figures 6.13 and 6.14 compare model results with experimentally measured slab temperatures for two different cooling cycles. Figure 6.13 gives results for the ambient cooled case for both the $34-\mathrm{mm}$ - and $40-\mathrm{mm}$-thick slabs. In this instance, results from the lumped-mass model are used since it properly accounts for the effect of upstream cassette components. Clearly evident is the excellent agreement between model and experiment after the first hour of recovery. Also evident, however, is as much as a $20 \%$ difference seen in the early stages of the recovery process. This early-time disagreement has not been resolved, with possible sources being incorrect values for the blastshield starting temperature, incorrect blastshield and slab emissivities, or errors in the experimental data. This discrepancy may be explored further in follow-on work.

An example of chilled-gas recovery is given in Figure 6.14. In this instance, the cooling gas inlet temperature was reduced by $0.5^{\circ} \mathrm{C}$ for the first 2.6 hours of recovery, followed by rewarming to ambient conditions. Figure $6.14 \mathrm{~b}$ gives the comparison 
between model and experimental results. Again the agreement is excellent except for the first hour of recovery. Also shown on this figure is the corresponding ambient cooled model profile. It is seen that with a mere $0.5^{\circ} \mathrm{C}$ reduction in the cooling fluid temperature, recovery is decreased by as much as 2 hours. This enhancement is due to the nearly doubling of the radiation exchange temperature difference during much of the recovery cycle. Because of this enhancement, chilled-gas cooling is a baseline feature of the NIF design.

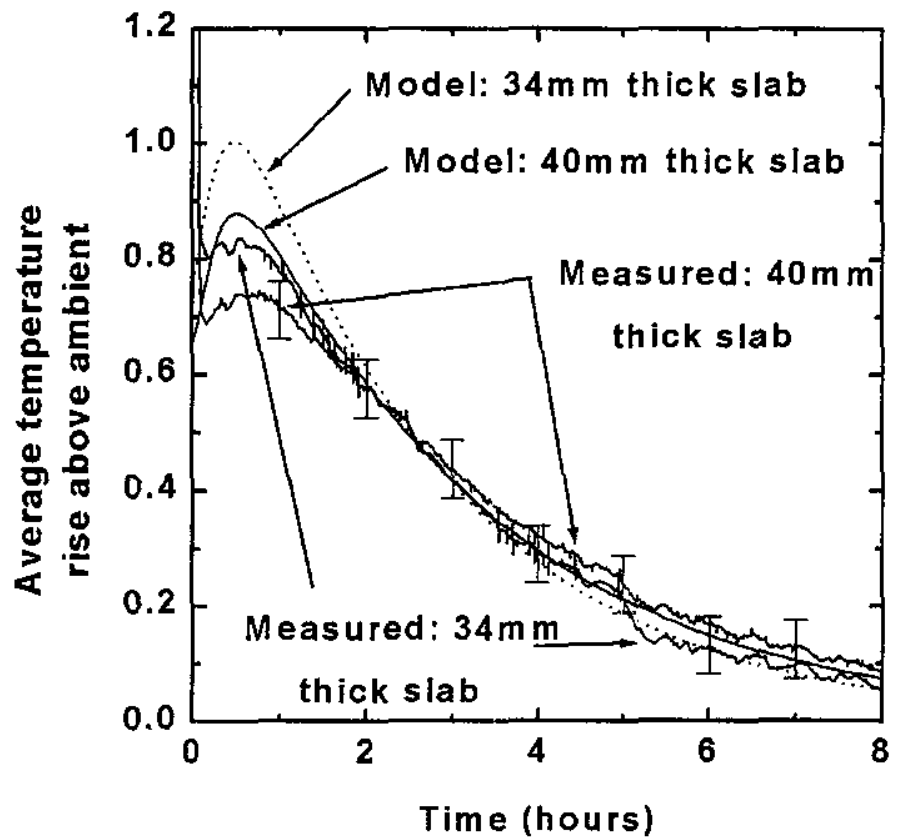

Figure 6.13. Comparison of model predictions of the slab average temperature rise to AMPLAB measured values. Results are given for both $34 \mathrm{~mm}$ thick and $40 \mathrm{~mm}$ thick slabs.

To allow use of uncooled AMPLAB data, the lumped-mass model was extended to accommodate stagnant cooling conditions. Natural convection coupling of energy from the flashlamps to the blastshield and reflectors was incorporated. Because of the absence of parasitic side-wall losses in the thermal model, this model adjustment is most accurate for end-slab geometries where radiation from the glass elements to the beamtube structure is the primary system energy loss mechanism. Figure 6.15 gives a comparison of model results to experimentally measured slab average temperatures for the amplifier diamond configuration. The agreement is reasonably good during the entire recovery cycle. Clearly evident, however, is stratification in the system due to the buoyantly driven convection currents in the flashlamp and slab cavities. During the early stages of recovery, there is a clear increase in temperature in going from the bottom aperture (" $D$ ") to the top aperture (" $A$ "). This is likely to be primarily due to stratification in the flashlamp cassettes, which leads to top-to-bottom variations in the blastshield temperature. 
$\dot{\mathrm{V}}$
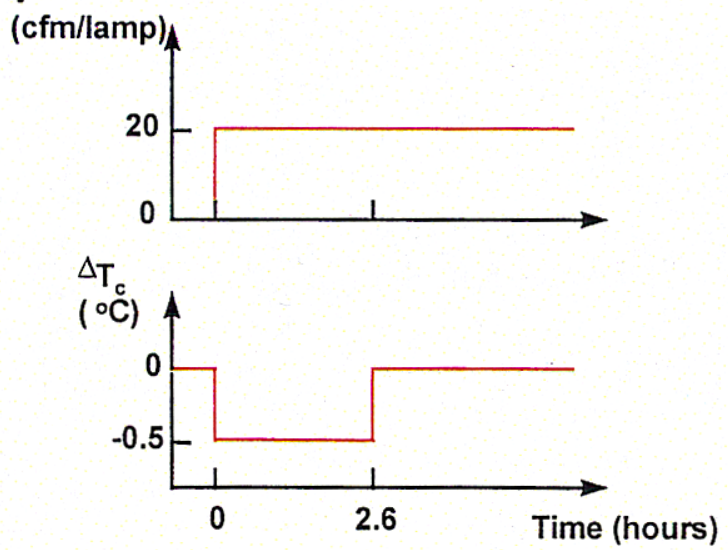

(a)

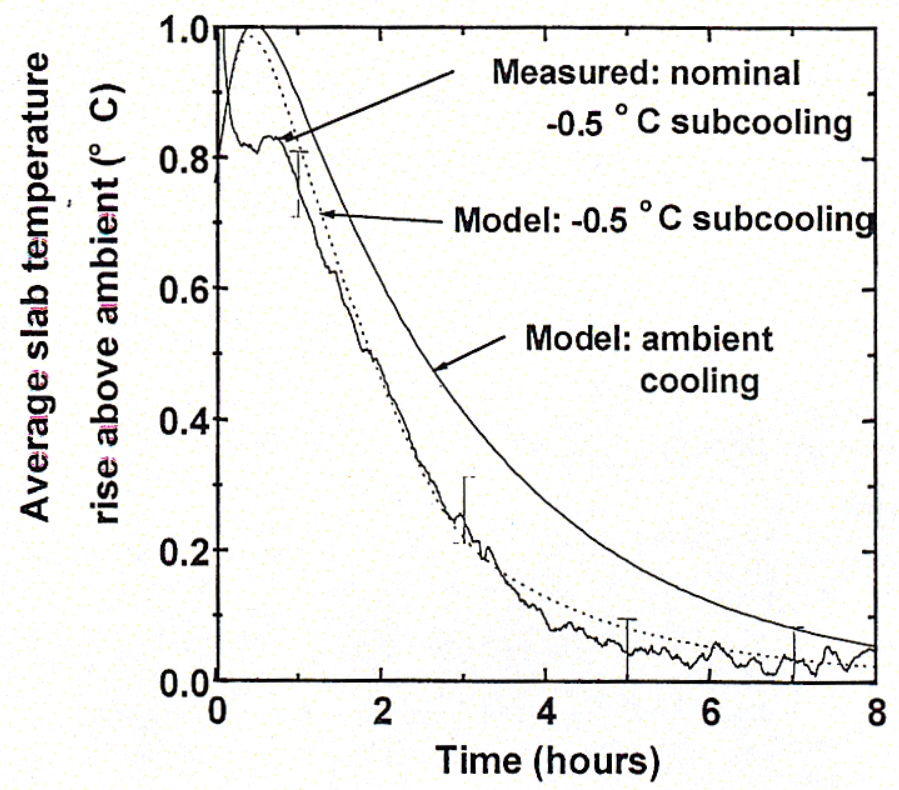

(b)

Figure 6.14. Temperature recovery of the AMPLAB slabs using chilled cooling gas in the flashlamp cassette. (a) Temperature and flow velocity profiles used in the numerical simulations of chilled gas cooling. This is the nominal profile used in the AMPLAB experiment. (b) comparison of calculated and measured slab temperatures.

\subsection{Laser slab wavefront recovery}

Sample model results of the three-dimensional thermo/mechanical/optical calculation suite are given in Figures 6.16 through 6.18. Figure 6.16 shows the temperature distribution on one of the large faces of the laser slab, after 8 hours of cooling with ambient temperature gas after a shot. The central iso-line is for a temperature $0.1075^{\circ} \mathrm{C}$ above ambient. The iso-line increments are $0.0025^{\circ} \mathrm{C}$. The hottest temperatures are along the top and bottom edge claddings that are not as thermally well heat-sunk as the side regions. The left-to-right temperature asymmetry is due to the orientation of the slab with respect to the blastshields. One end of the face is more closely coupled to the facing blastshield than the other end. The corresponding slab distortion is shown in Figure 6.17. Because of cooling rate differences between the central and side cassettes, the slab is warped into the shape shown. The final step in the sequence is the evaluation of the 
optical distortions. Figure 6.18 shows the tilt-corrected phase distribution across the aperture. The tilt-corrected quantity is of interest in this investigation since the thermal recovery criteria addressed in Section 6.1 relates to deformable mirror limits, which are associated with non-tilt components of the total wavefront error. Note that the optical distortion is low order, thus amenable to deformable mirror correction.

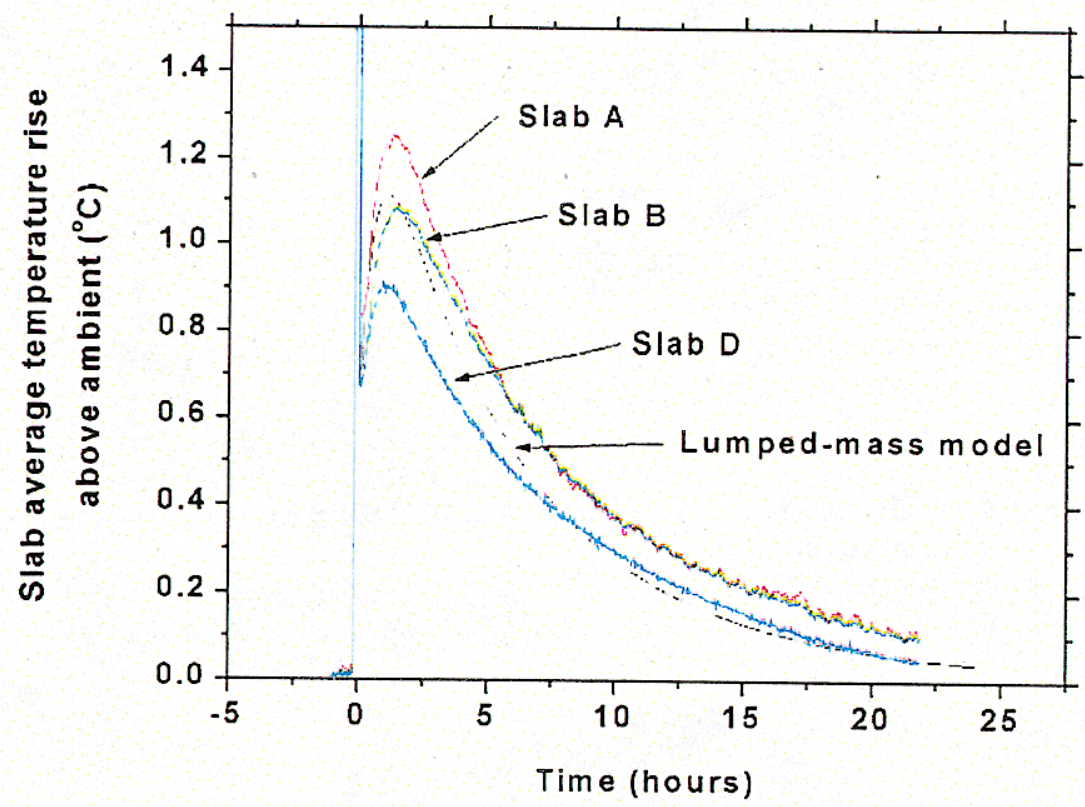

Figure 6.15. Comparison of measured and calculated slab temperatures for the case of passive cooling of the flashlamp cassettes. Because the lumped-mass model lacks spatial detail, the differences between the top and bottom slabs could not be modeled. In the experiments, the A and D slabs were $34 \mathrm{~mm}$ thick, while the B slab was $40 \mathrm{~mm}$ thick.

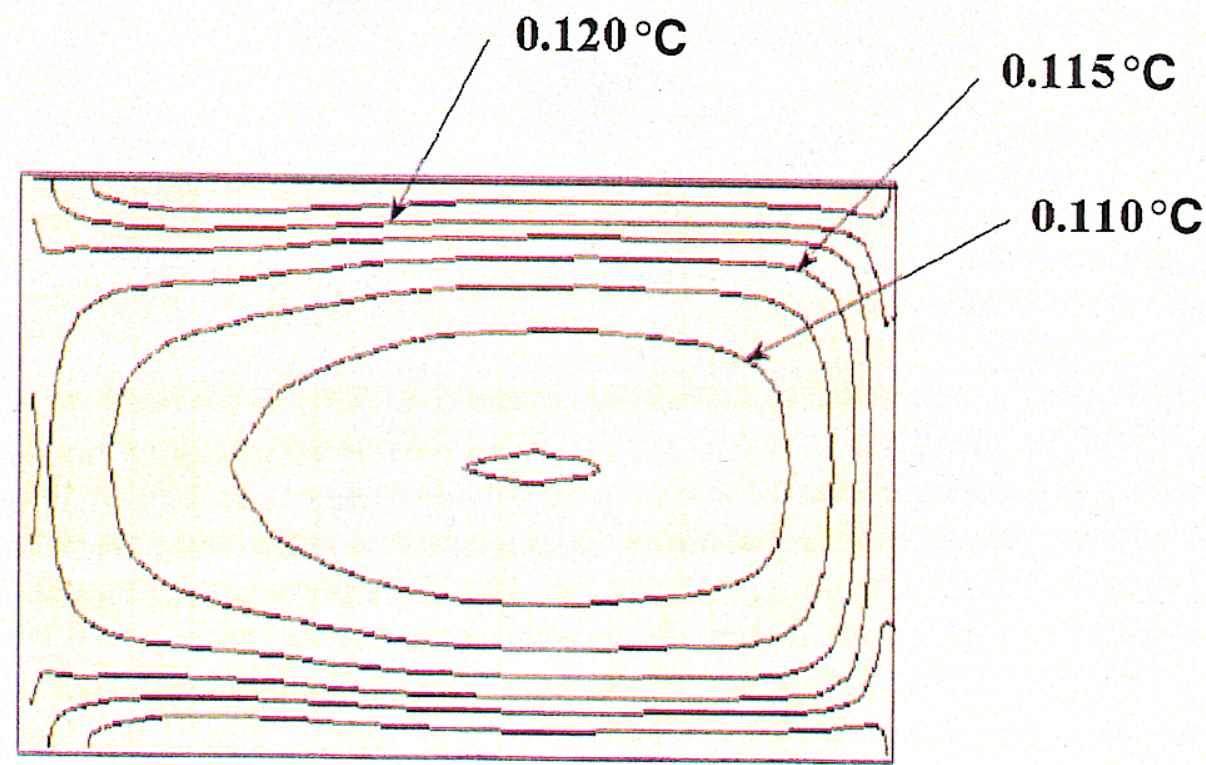

Figure 6.16. Temperature contours on one face of the laser slab. The contours are spaced at an increment of $0.0025^{\circ} \mathrm{C}$. Note the left-to-right asymmetry in the temperature field due to the orientation of the slab with respect to the blastshields. 


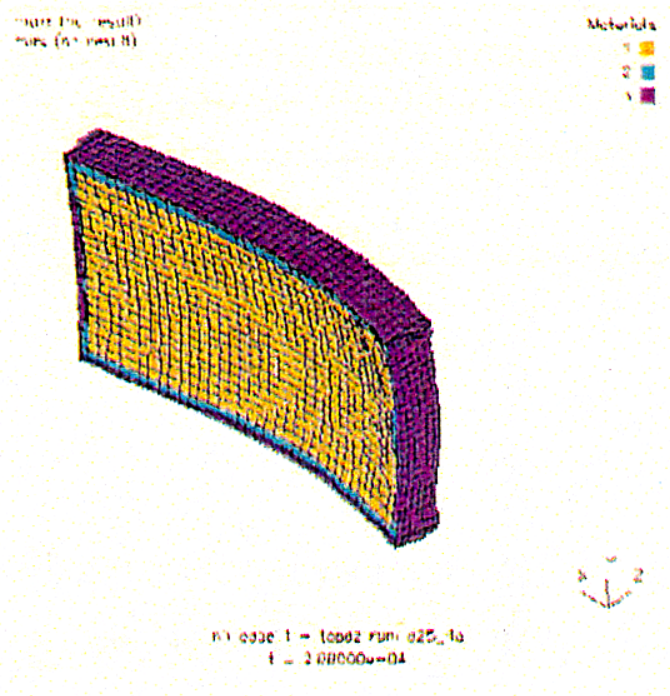

Figure 6.17. The deformed central slab as viewed from the central flashlamp cassette. Deformations are multiplied by $10^{6}$ in order to produce this image.

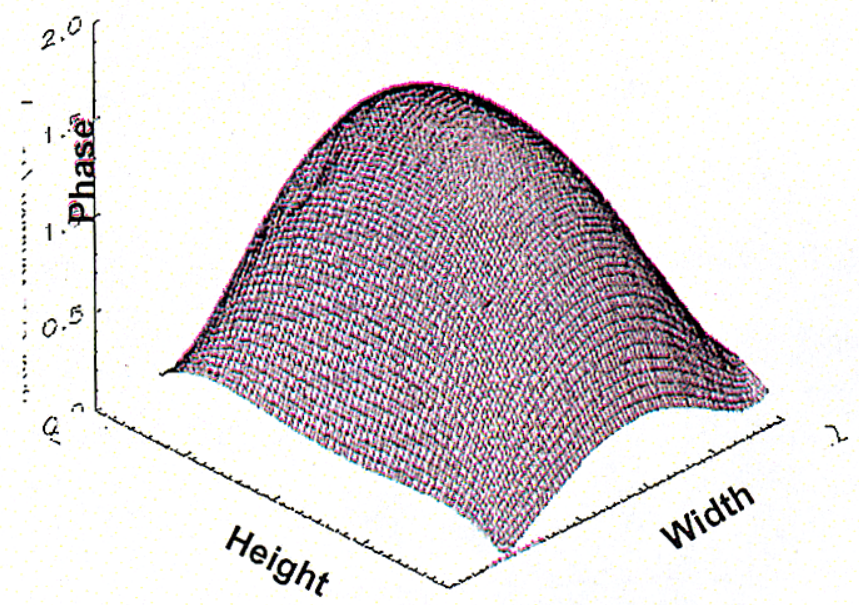

Figure 6.18. Representative distortion phase distribution resulting from temperature gradients and mechanical distortion of an interior slab.

In the early stages of measuring wavefront recovery in AMPLAB, thermal recovery trends were difficult to quantify because of system variations that dominated the thermal contributions. To overcome these difficulties, the flashlamps were fired four times within 12 minutes to enhance the optical distortions. Measurements were made for both cooled and uncooled recovery conditions in both the " $\mathrm{A}$ " and " $\mathrm{C}$ " apertures. A comparison of model and measured results was then made. Results are presented in Figure 6.19. The data is for ambient gas-cooled recovery of aperture " $\mathrm{A}$ ". The slab distortions are experimentally extracted from the data in a fashion depicted in Figure 6.20. Prior to the flashlamp firing, and at one-hour increments thereafter, sets of 20 interferograms were taken. Within each set, the resulting wavefronts were averaged, with the intent of reducing the gas contributions by nominally the root of the number of samples. These 
averages were then subtracted to extract the change in low-order optical aberrations that we associate with the laser slabs. There were significant magnitude and shape differences between the measured and calculated wavefronts, due likely to the remaining gas motion effects in the measured values as well as possible experimental contributions from other optical elements in the system. For that reason we select to simply represent the optical distortion in terms of the P-V magnitude. Referring back to Figure 6.19, it was necessary to multiply the model P-V magnitude prediction by a factor of 3 to bring it into agreement with the measured $\mathrm{P}-\mathrm{V}$ magnitude.

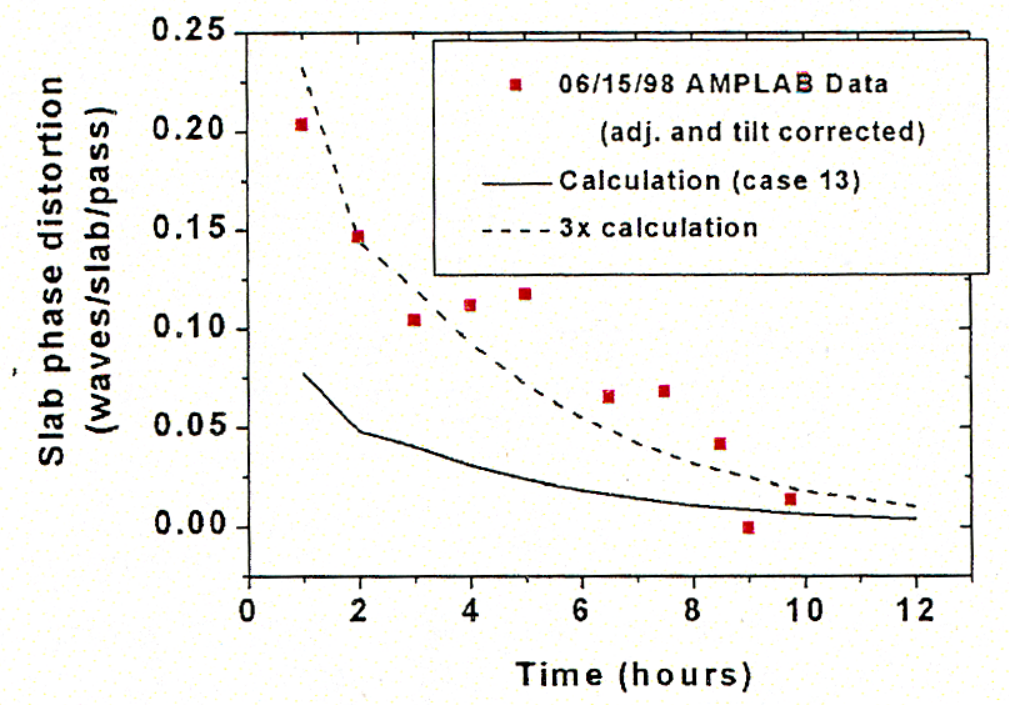

Figure 6.19. The comparison of AMPLAB-measured slab distortions to calculated distortions for a 4-shot case. The model results were found to differ by a factor of 3 from the measured.

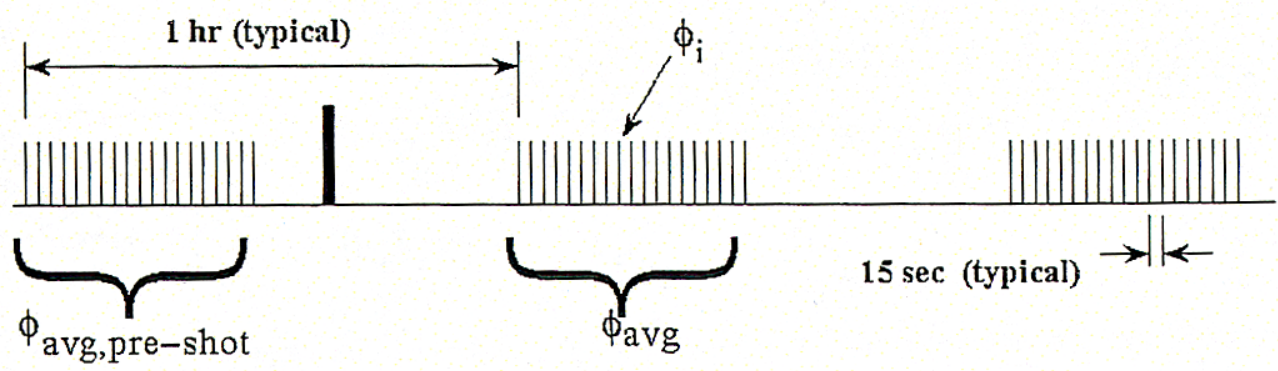

$$
\begin{aligned}
& \phi_{\text {avg }}=\frac{1}{N} \sum_{i=1}^{N} \phi_{i} \\
& \phi_{\text {slab }}=\phi_{\text {avg }}-\phi_{\text {avg,pre-shot }} \\
& \phi_{\text {g a s }}=\phi_{i}-\phi_{\text {avg }}
\end{aligned}
$$

Figure 6.20. Characterization of the wavefront acquisition and processing approach used to extract slab and gas distortion information in AMPLAB. 
We have performed mesh and boundary condition sensitivity studies on the numerical model and found no credible numerical source for these discrepancies. [6.6] With insulated slab edges, we could drive the optical distortions to near the measured value, but as discussed earlier this is not a credible condition in light of the thin air gaps between the slab and masks. As mentioned above, a more likely source is in the experimental measurements. Since average wavefronts that compose the difference may be taken several hours apart, imbedded in this measurement are thermal effects on the end mirrors and other optical elements in the system. For example, consider Figure 6.21, which gives the measured optical distortions for aperture " $\mathrm{A}$ " cooled and uncooled recovery and aperture " $C$ " cooled recovery. Focusing on the aperture " $A$ " results, there is a common decay rate after about the first hour of recovery. This indicates the potential for dominance of something else in the system that is not thermally affected by the presence of the cooling flow. Further numerical and experimental investigation is required to fully identify and quantify these contributions.

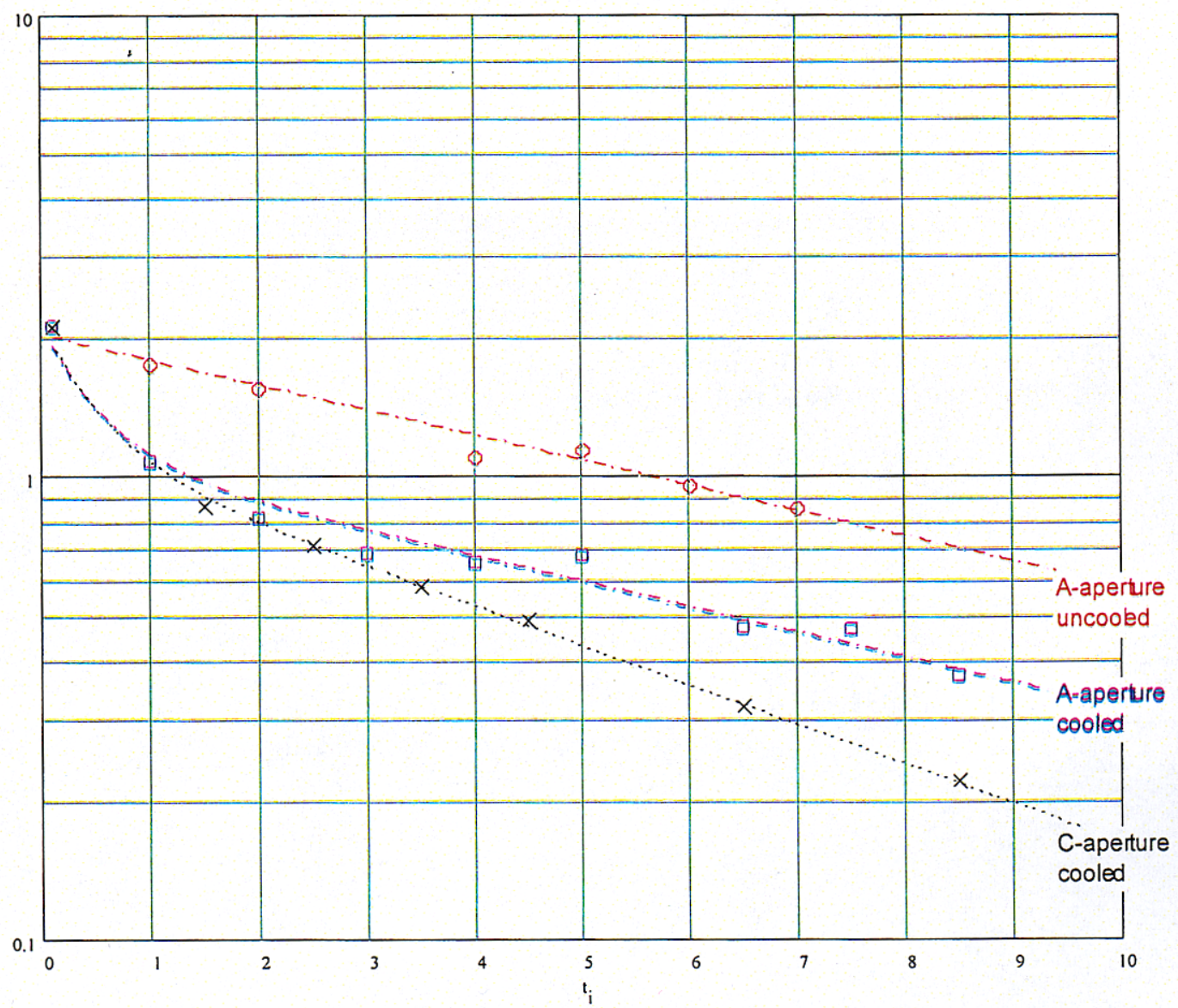

Figure 6.21. Peak-to-valley wavefront distortion for cooled and uncooled tests in apertures $\mathbf{A}$ and $\mathbf{C}$. Note the common late-time decay rate in the two aperture $A$ tests, which denotes a thermal effect not associated with the cooling gas that has an important effect in the system.

With this uncertainty in mind, predictions of the tilt-corrected P-V slab phase distortion for the NIF are presented in Figure 6.22. The numerical results are bounded by an aggressive assumption, in which we assume the numerical model results are correct, 
and a conservative assumption in which we apply the $3 \times$ multiplier discussed above. Applying these assumptions, the predicted P-V slab distortion for the entire multipassed beamline is $0.4 \pm 0.2$ waves after 7 hours, and $1.5 \pm 0.7$ waves after 3 hours. It is clear that with respect to a 7-hour recovery, there is adequate margin to feel confident that slab optical distortions will be acceptable at the end of the thermal recovery period. However, for an accelerated shot-rate scenario, which requires recovery in three hours, the conservative assumption-based estimates are marginal.

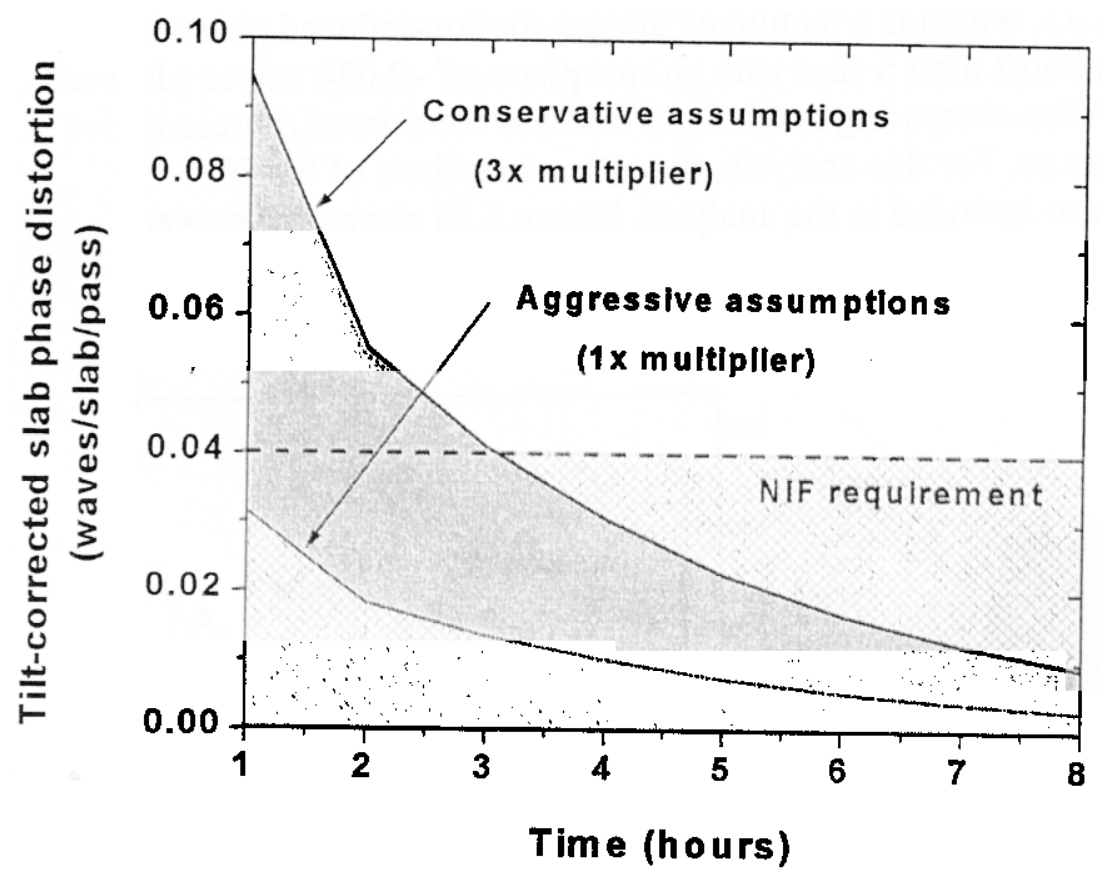

Figure 6.22. Predictions of the NIF slab distortions. Conservative and aggressive assumptions are applied in order to bracket the problem. The NIF requirement of 0.04 waves/slab/pass ( 2.2 waves $\mathrm{P}-\mathrm{V}$ per beamline) is denoted on the figure.

\subsection{Gas distortion wavefront recovery}

When considering gas motion wavefront distortions, it is important to recognize that these effects are distributed throughout the entire length of the laser system. Thus, where there is a temperature imbalance to drive convective currents, there will be a source of distortion. Therefor, account must be taken of effects in the amplifier itself as well as in the adjacent beamtubes. Because of the distributed nature of these distortions, scaling of available experimental results from AMPLAB and Beamlet becomes a critical aspect of the exercise. This required detailed propagation studies of the full NIF optical train as well as development of a projection model to relate AMPLAB and Beamlet data to the NIF system. A major goal of this effort was to provide a relationship between system optical performance, from the standpoint of gas motion distortions, and system temperatures, which are easily calculated and measured.

As a starting point, consider the gas motion optical distortions measured in AMPLAB and a projection of the NIF system requirements. The instantaneous gas distortions in 
$A M P L A B$ were extracted from the same wavefront data used for the slab distortions. In this instance, any one of the elements of the sets of 20 wavefronts can be subtracted from the average of the set (see Figure 6.20). In this manner, the slab distortions, which are much lower order than the gas phase distortions are subtracted out. An example of a resulting phase front is given in Figure 6.23. The rms values for the individual elements of the sets are plotted in Figure 6.24 for an uncooled four-shot recovery case. Note that the decay to the baseline value occurs in about eight hours in this instance.

Refer again to Figure 6.23, which is a picture of the gas-motion-induced phase noise on the AMPLAB beam ten hours after a shot with an rms phase of $\sim 0.034$ waves (data set $0615 \mathrm{D}$, cooling fans off). After comparing much data, this was considered representative of the gas disturbance signature. For this analysis, two other snapshots of the 20 (randomly selected) were also included in the analysis. Figure 6.25 shows the power spectral density curves for the three measurements.
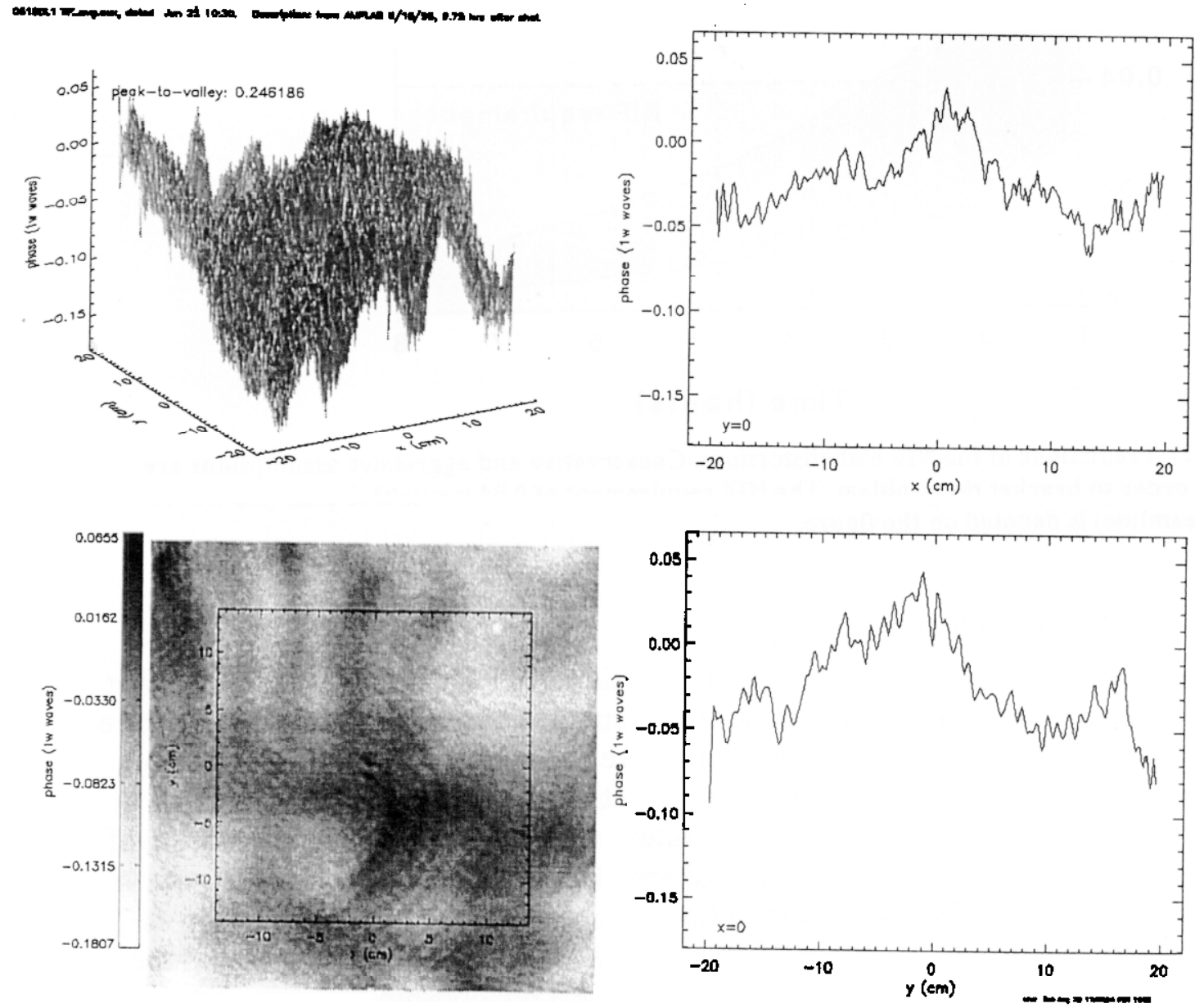

Figure 6.23. Turbulence phase from AMPLAB measurement.

To consider the effect on NIF spot size due to this phase noise, the phase of each of these disturbances was added separately to a simulated $1.05-\mu \mathrm{m}$ NIF beam just entering 
the frequency converter (1-ns, high-power pulse). In actuality, of course, this noise would be added in a distributed fashion in the amplifiers during the multiple NIF passes. Since the specification considers only the $80 \%$ spot size, however, which is largely dictated by long-scale aberrations that remain principally as phase in the well-relayed NIF laser, it is largely irrelevant to the spot size whether such noise is added in a distributed fashion along the beam paths or at the end of the chain. The files were also low-pass frequency filtered at $100 \mu \mathrm{rad}$ before being added to the beam to simulate the effect of pinholes (this made little difference to the results). The adaptive optic model in PROP92 [6.8] was also applied against the beam after addition of the file, under the aggressive assumption that the adaptive optic loop is fast enough to correct the air turbulence. This correction made little difference to the spot size, however, due to the fairly small scale lengths of the phase noise. Finally, a scaling factor was included to scale the amplitude of the phase up and down from the values in the AMPLAB measurements.

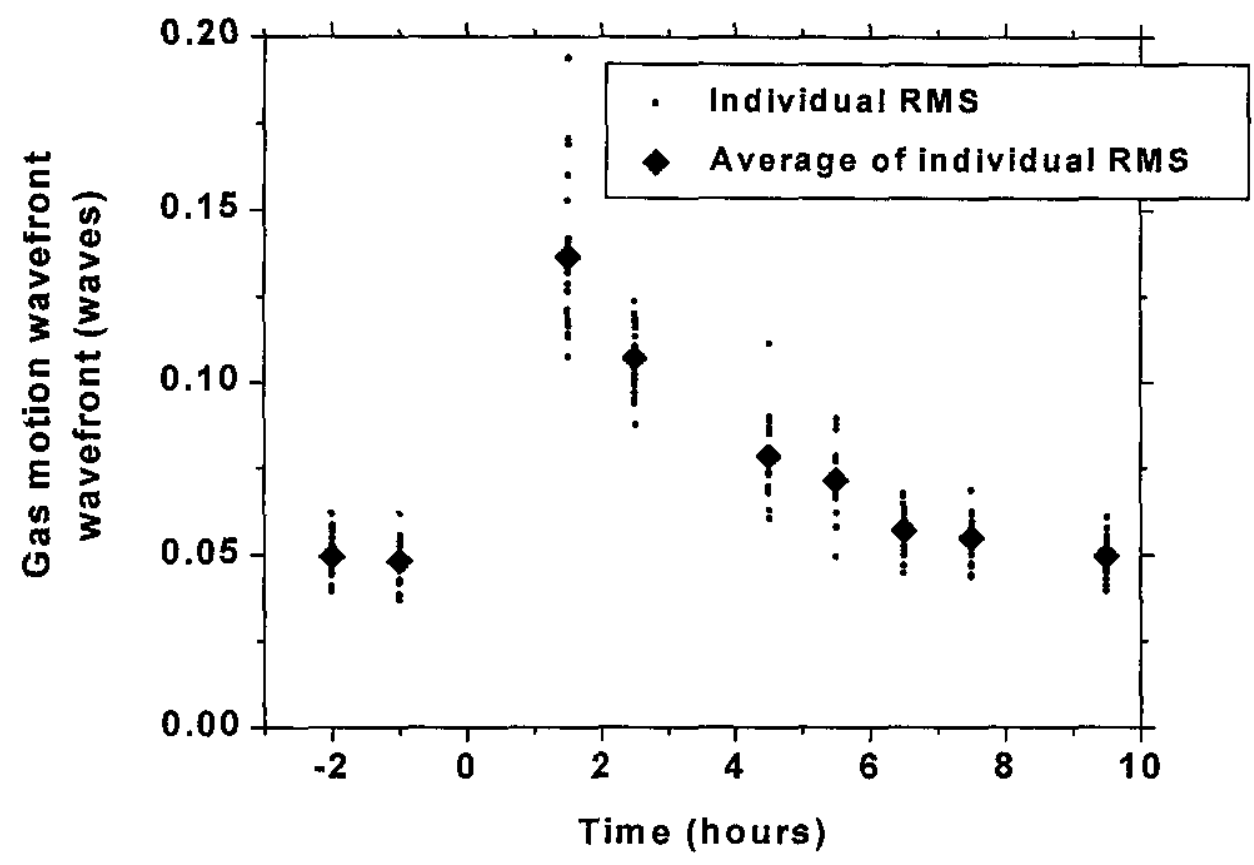

Figure 6.24. Rms values of the phase distortion for the individual wavefronts taken in each data set. Also shown is the average of the 20 wavefronts in each set. 


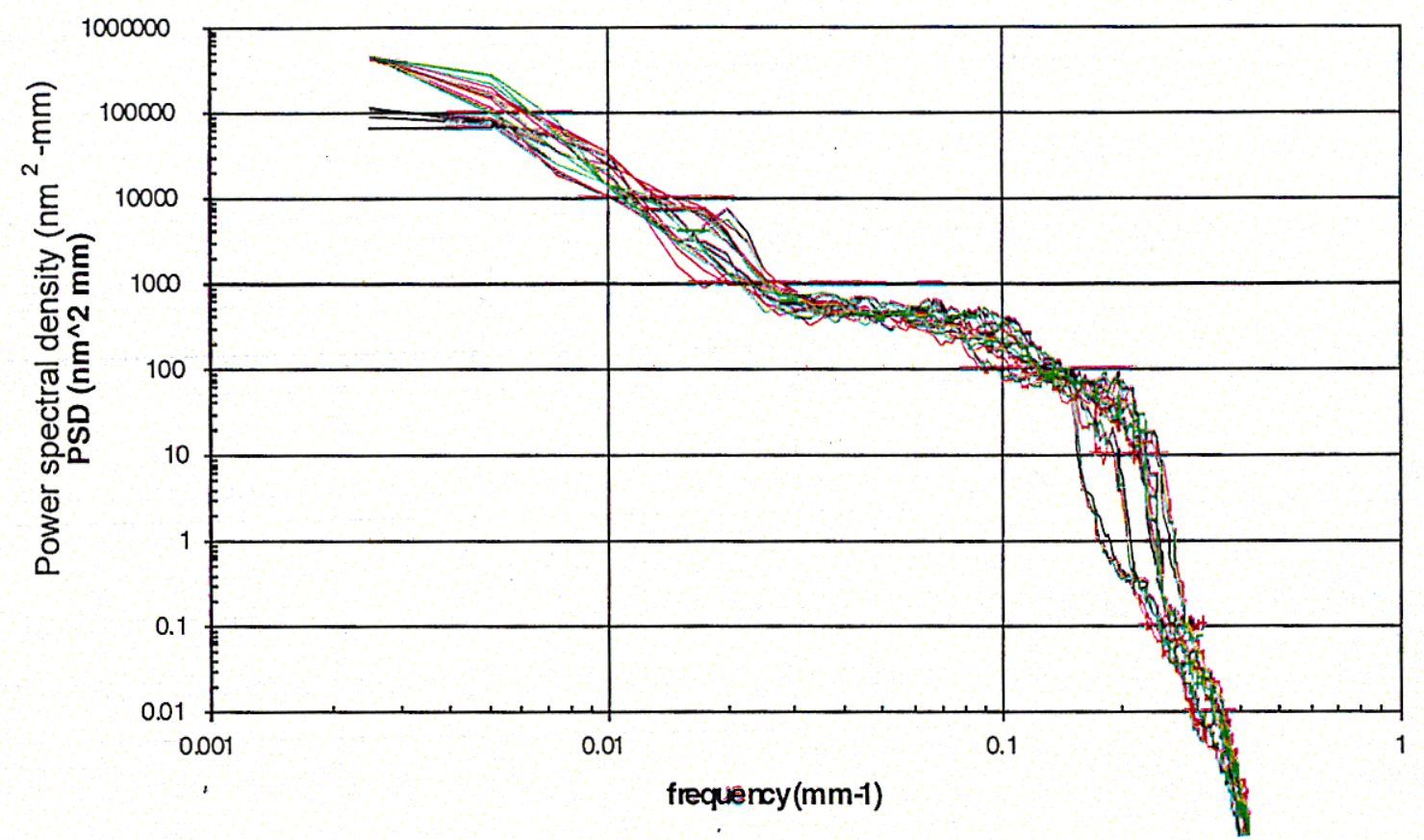

Figure 6.25. Power Spectral Density (PSD) curve for the three measured files. This figure shows a 1-D collapse of the 2-D PSD at six different angles for each of the three measurements. This representation is preliminary, in that a detailed analysis has not been done of the spectral range of validity.

Figure 6.26 shows the spot size as a function of the phase scaling factor for the three cases, including both filter and adaptive optic corrections. These results indicate that if the AMPLAB measurements are scaled up by a factor of 3 to 5 , the $5-\mu \mathrm{rad}$ limit is exceeded. Based on the minimum multiplier, this translates to a NIF system gas phase distortion allowance of nominally 0.1 waves rms. What is lacking to this point, however, is quantification of this phase-amplitude scaling factor, particularly as it relates to calculated and observed temperatures in the system. Quantification of the relationship between temperature and phase distortion is a necessary step since system temperatures are easily calculated and measured.

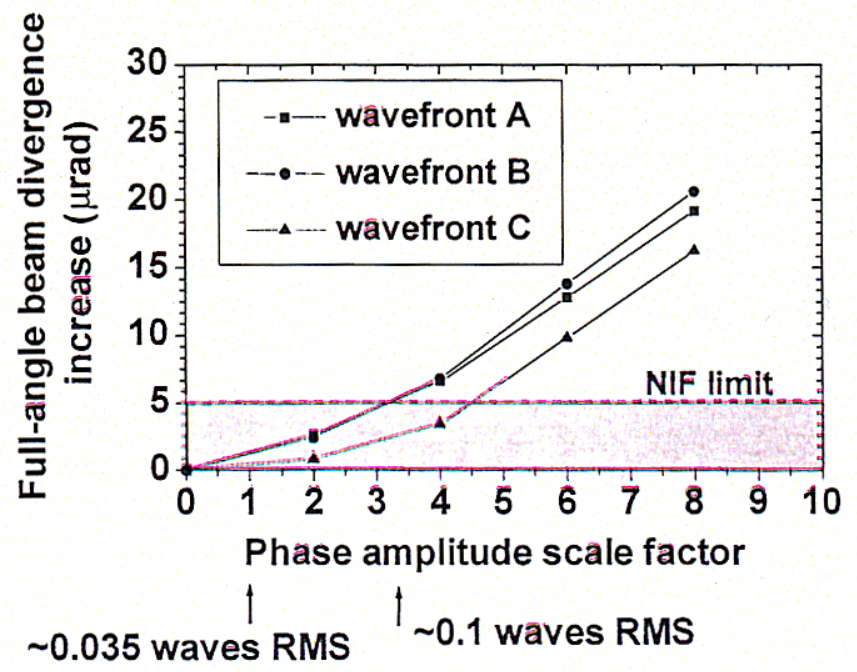

Figure 6.26. Increase in $80 \%$ full-angle spot size with scale factor for the three AMPLAB measurements. The scale factor acts as a multiplier on the data rms phase distortion of $\sim 0.34$ waves. 
To develop this relationship, we consider gas motion optical data taken on both AMPLAB and Beamlet. In Beamlet data taken in 1996, it was observed that the focal spot size correlated well with two temperature quantities: (1) an "internal" temperature difference that most likely controls convective flows in the internal cavities in the amplifier chain and (2) an "end" temperature difference that likely controls convective flow in the beamtubes. This is depicted in Figure 6.27. This same observation was noted in AMPLAB data, as shown in Figure 6.28. Again, the gas phase distortions correlate well with the slab temperature rise, which in this instance represents the "end" temperature difference. This is appropriate since the AMPLAB configuration is only two slabs long, and thus, is likely to be dominated by beamtube disturbances. The offset problem at low temperature values is likely due to system effects outside the domain of thermal recovery.

It must be emphasized that both the AMPLAB and Beamlet gas distortion data are integrated optical effects of their respective systems, individually providing little information about scaling. The scaling link is provided through a simple construct model that employs the available data to specify adjustable parameters. The goal of this model construct is to relate system convection current driving temperature differences to a system rms phase distortion, and then through Figure 6.26, to the added beam divergence. The model is based on the premise that gas distortions can be separated into interior and end contributions as depicted in Figure 6.29. Furthermore, from the observations noted earlier, the distortions scale linearly with characteristic temperature differences. Thus we may say that

$$
\begin{aligned}
& \phi_{\mathrm{i}}=\mathrm{K}_{\mathrm{i}} \Delta \mathrm{T}_{\mathrm{i}} \\
& \phi_{\mathrm{e}}=\mathrm{K}_{\mathrm{e}} \Delta \mathrm{T}_{\mathrm{e}}
\end{aligned}
$$

where $\phi$ is the local phase distortion, $\mathrm{K}$ is the proportionality constant, and $\Delta \mathrm{T}$ is the temperature difference. The " $\mathrm{e}$ " and "e" subscripts denote the interior and end regions respectively. In Eq. (6.1), we provide for different temperature differences in the interior and end regions. For cases where the flashlamps are cooled with ambient temperature gas, we expect the temperature differences to be equivalent after the first hour of recovery. However, in an uncooled case, calculations and experimental data indicate that there will be a equilibration of the interior temperatures at an elevated value, maintaining an end temperature difference, In the application of Eq. (6.1), it is hypothesized that the proportionality constants are the same for AMPLAB and the NIF, because the geometry heights are the same, but different for Beamlet. In Beamlet, the amplifier cavities are two slabs tall. Thus, we stipulate that

$$
\begin{aligned}
& K_{i}=K_{i, n}=K_{i, a}=\frac{K_{i, b}}{\beta} \\
& K_{e}=K_{e, n}=K_{e, a}=\frac{K_{e, b}}{\beta}
\end{aligned}
$$


where the additional subscripts "n", "a", and "b" are used to denote NIF, AMPLAB, and Beamlet respectively. The $\beta$ factor is used to account for the Beamlet scaling differences. For example, a $\beta$ factor of 0.5 assumes that the NIF system is twice as sensitive to temperature differences as the Beamlet system, which has been previously assumed.

(a)

(b)
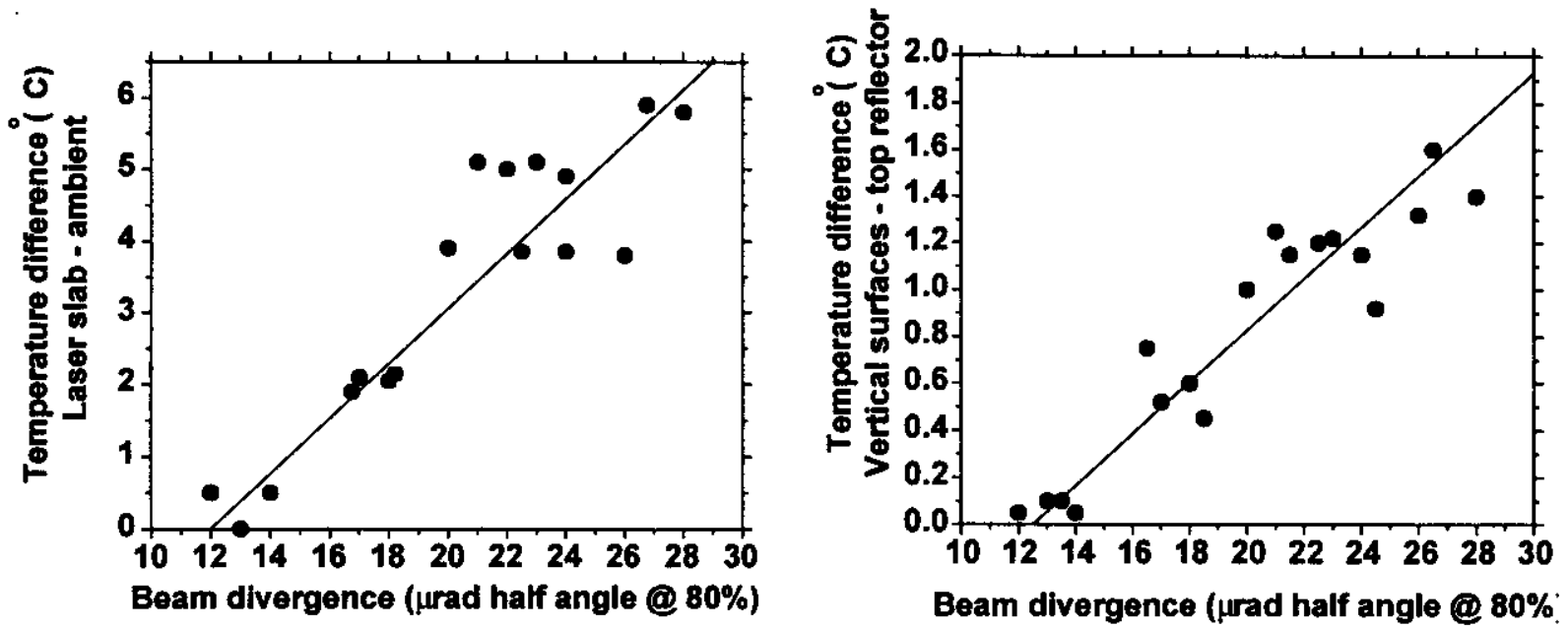

Beam divergence ( $\mu$ rad half angle @ $80 \%$.

Figure 6.27. Temperature correlation observed in the Beamlet focal spot data (a) correlation with an end-cavity driving temperature difference-the difference between the slab temperature and ambient (the beamtube) temperature and (b) correlation with an interior-cavity driving temperature difference-the difference between the vertical surface temperatures (average temperature of laser slabs and blastshields) and the top reflector temperature.

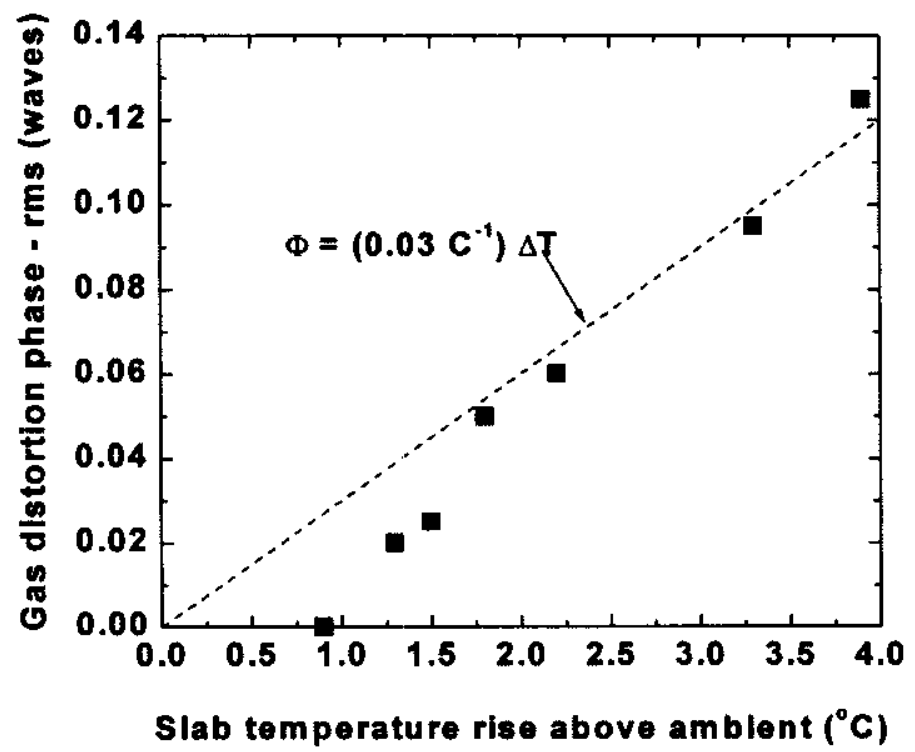

Figure 6.28. Temperature correlation observed in the AMPLAB gas distortion data. 


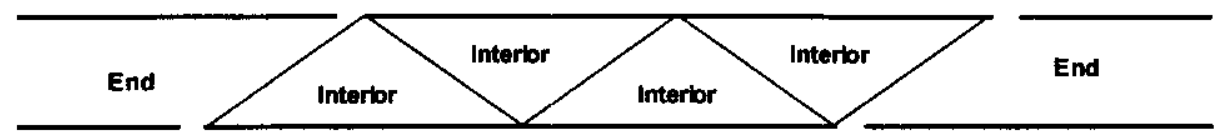

Figure 6.29. A depiction of the two region types incorporated in the simple gas-distortion-scaling model. AMPLAB consisted of 1 interior and 2 end regions. The NIF main amplifier consists of 9 interior and 2 end regions.

Given these assumptions, model equations were constructed for the total system optical distortion, assuming the proper coherent and incoherent additions. Measured AMPLAB and Beamlet distortions were then used to extract the model constants.

For AMPLAB, which was two slabs long and double passed, a single interior region was present in addition to the two end regions. The model equation for the system phase distortion $(\Phi)$, accounting incoherent and coherent contributions in the end and interior regions, is

$$
\Phi_{a}^{2}=\underbrace{2\left(2 \cdot \phi_{e, a}\right)^{2}}_{\text {End regions }}+\underbrace{\left(2 \cdot \phi_{i, a}\right)^{2}}_{\text {Interior cavity }}
$$

In Eq. (6.3), the factor of 2 multiplier inside the parenthesis accounts for the 2 pass coherent addition, while multipliers outside the parenthesis account for incoherent addition of multiple regions. Substituting Eqs. (6.1) and (6.2) into Eq. (6.3)

$$
\Phi_{a}^{2}=8 \cdot \mathrm{K}_{\mathrm{e}}^{2}\left(\Delta \mathrm{T}_{\mathrm{e}, \mathrm{a}}\right)^{2}+4 \cdot \mathrm{K}_{\mathrm{i}}^{2}\left(\Delta \mathrm{T}_{\mathrm{i}, \mathrm{a}}\right)^{2}
$$

For Beamlet, which employed a 11 slab long 4 pass main amplifier and a 5 slab long single pass power amplifier, the model construct is

$$
\begin{aligned}
\Phi_{b}^{2} & =\underbrace{2\left(4 \cdot \phi_{e, b}\right)^{2}}_{\begin{array}{l}
\text { Main Amp. } \\
\text { end regions }
\end{array}}+\underbrace{10\left(4 \cdot \phi_{i, b}\right)^{2}}_{\begin{array}{c}
\text { Main Amp. } \\
\text { interior reg. }
\end{array}}+\underbrace{2\left(\phi_{e, b}\right)^{2}}_{\begin{array}{c}
\text { Power Amp. } \\
\text { end regions }
\end{array}}+\underbrace{4\left(\phi_{i, b}\right)^{2}}_{\begin{array}{l}
\text { Power Amp. } \\
\text { interior reg. }
\end{array}} \\
& =34 \cdot \beta^{2} \cdot \mathrm{K}_{\mathrm{e}}^{2}\left(\Delta T_{e, b}\right)^{2}+164 \cdot \beta^{2} \cdot \mathrm{K}_{\mathrm{i}}^{2}\left(\Delta T_{i, b}\right)^{2}
\end{aligned}
$$

The NIF system employs an 11 slab long 4 pass main amplifier, and a 5 slab long double passed power amplifier. In this instance the construct is

$$
\begin{aligned}
\Phi_{n}^{2} & =\underbrace{2\left(4 \cdot \phi_{e, n}\right)^{2}}_{\begin{array}{c}
\text { Main Amp. } \\
\text { end regions }
\end{array}}+\underbrace{10\left(4 \cdot \phi_{i, n}\right)^{2}}_{\begin{array}{c}
\text { Main Amp. } \\
\text { interior reg. }
\end{array}}+\underbrace{2\left(2 \cdot \phi_{e, n}\right)^{2}}_{\begin{array}{c}
\text { Power Amp. } \\
\text { end regions }
\end{array}}+\underbrace{4\left(2 \cdot \phi_{i, n}\right)^{2}}_{\begin{array}{c}
\text { Power Amp. } \\
\text { interior reg. }
\end{array}} \\
& =40 \cdot \mathrm{K}_{\mathrm{e}}^{2}\left(\Delta T_{e, n}\right)^{2}+176 \cdot \mathrm{K}_{\mathrm{i}}^{2}\left(\Delta T_{i, n}\right)^{2}
\end{aligned}
$$


Remaining as parameters in these equations, besides temperature differences are the region temperature correlation parameters, $\mathrm{K}_{\mathrm{e}}$ and $\mathrm{K}_{\mathrm{i}}$, and the height scaling parameter $\beta$. To extract values for the temperature correlation parameters, a single set of Beamlet and AMPLAB data was employed so that Eqs. (6.4) and (6.5) could be simultaneously solved for the parameter values. The data employed follows:

$$
\begin{array}{ll}
\text { Beamlet: } & \left\{\begin{array}{l}
\Delta \mathrm{T}_{\mathrm{i}, \mathrm{b}}=0.5 \mathrm{C} \\
\Delta \mathrm{T}_{\mathrm{e}, \mathrm{b}}=3.5 \mathrm{C} \\
\Phi_{\mathrm{b}}=0.248 \text { waves }
\end{array}\right. \\
\text { AMPLAB: }\left\{\begin{array}{l}
\Delta \mathrm{T}_{\mathrm{i}, \mathrm{a}}=0.5 \mathrm{C} \\
\Delta \mathrm{T}_{\mathrm{e}, \mathrm{a}}=3.5 \mathrm{C} \\
\Phi_{\mathrm{a}}=0.102 \text { waves }
\end{array}\right.
\end{array}
$$

The Beamlet data consists of measured system optical distortion and measured temperature differences, taken on 02/07/97 (shot number B7020721). On this particular Beamlet sequence, the flashlamps were fired twice with the data taken approximately 5 hours after the first system shot. The AMPLAB optical distortion data was from the 06/12/98 4-shot data set, and selected at a time that produced an end temperature difference $\left(\Delta \mathrm{T}_{\mathrm{e}}\right)$ equivalent to the Beamlet data. This 4-shot uncooled case was selected because of the enhanced signal resulting from the hotter temperature state, as well as the elimination of spurious gas motion disturbances that would be present if the cooling fans were engaged. Since thermocouples were not in place for this particular data set, the temperatures were obtained using the lumped-mass model. The value $\Delta T_{i}$ given in Eq. (6.8) is a slight adjustment from the calculated value, and was required to get a solution from the coupled equations. It is likely fortuitous that it is equivalent to the Beamlet data set value. Further details of the model, and values for the temperature correlation parameters are given in Ref. 6.9. One observation, addressed in detail in Ref. 6.9, is that the mathematical result of a $\beta$ factor of 0.5 is a heavier weighting of interior regions that with a $\beta$ factor of unity is applied.

Results of the model, as applied to AMPLAB measured distortions are given in Figures 6.30 and 6.31. The corresponding temperature predictions from the lump-mass model are given in Figure 6.32. Consider first Figure 6.30, which is for passive flashlamp cooling (temperatures given in Figure 6.32a). Results agree equally well for $\beta$ factors of 0.5 and 1.0. Referring to Figure 6.32a, note the long decay time for the passive flashlamp cooling case. This demonstrates the importance of flashlamp cooling on amplifier thermal recovery. The origin of the "dip" in the $\beta=0.5$ curve at 1.5 hours is seen in the Figure 6.32a temperatures. As mentioned earlier, the interior cavities are weighted heavier with this value of $\beta$ than with a value of unity. The observed behavior is due to the crossover of the blastshield and slab temperatures, which form the interior cavity temperature difference. 


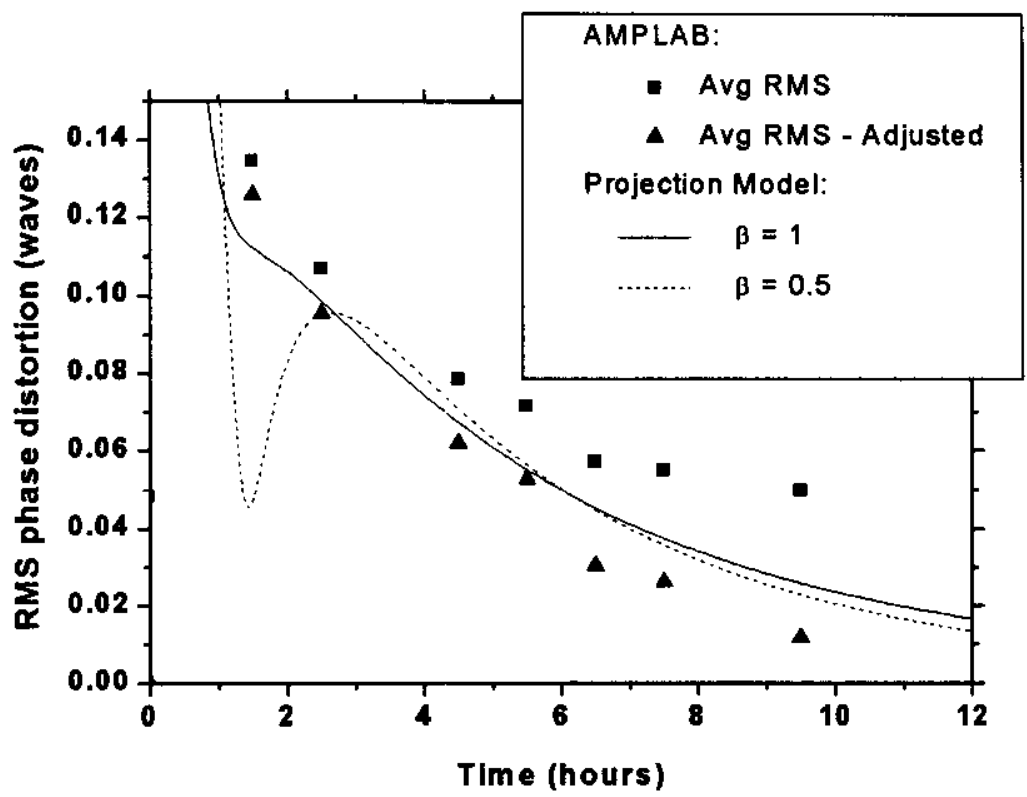

Figure 6.30. Comparison of projection model predictions to the AMPLAB data for passive flashlamp cooling. Both height scaled and unscaled predictions are given.

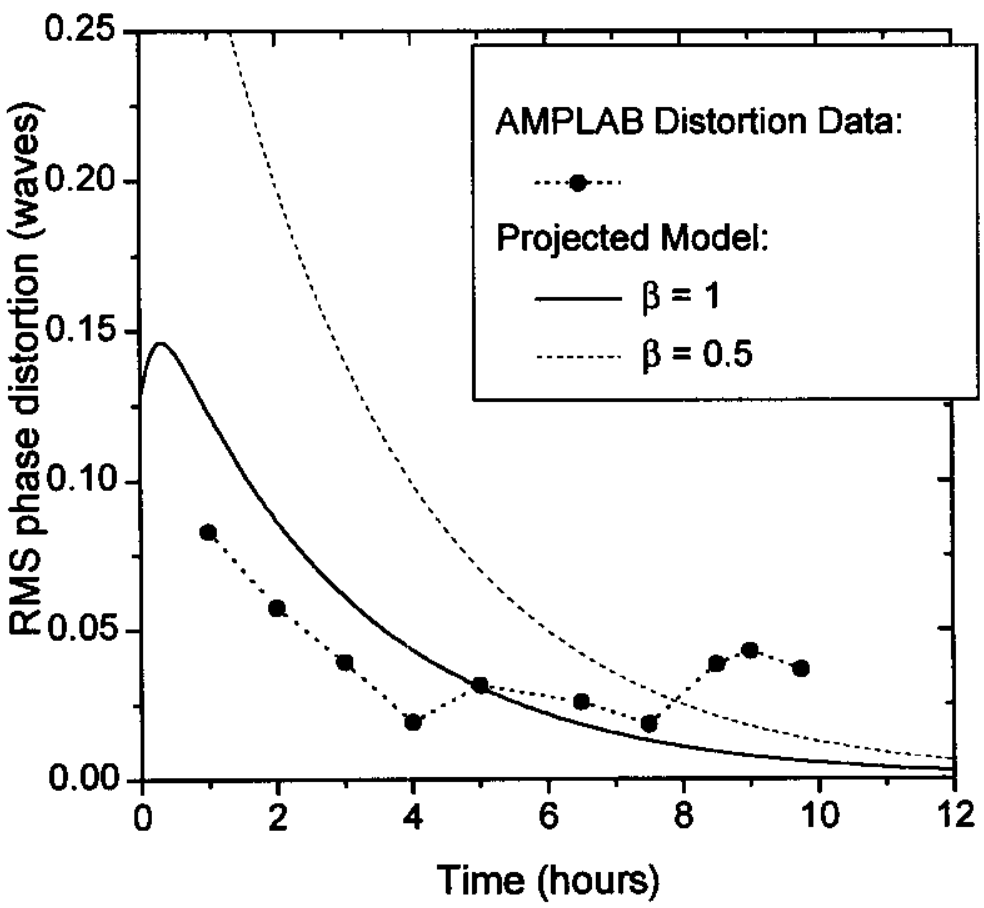

Figure 6.31. Comparison of projection model results to AMPLAB data for active flashlamp cooling. Predictions are presented for both the height scaled and unscaled cases. The corrected AMPLAB data attempts to correct for cooling system effects caused by the leaky blastshields. 


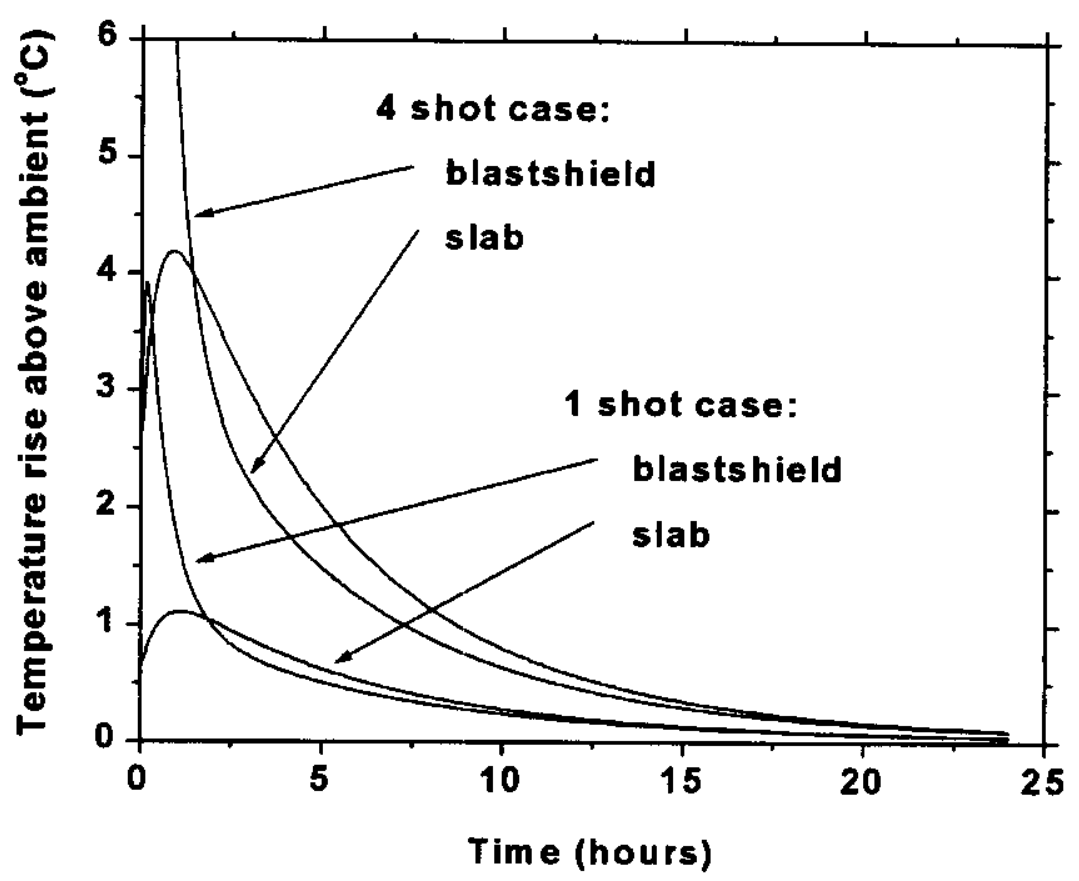

(a)

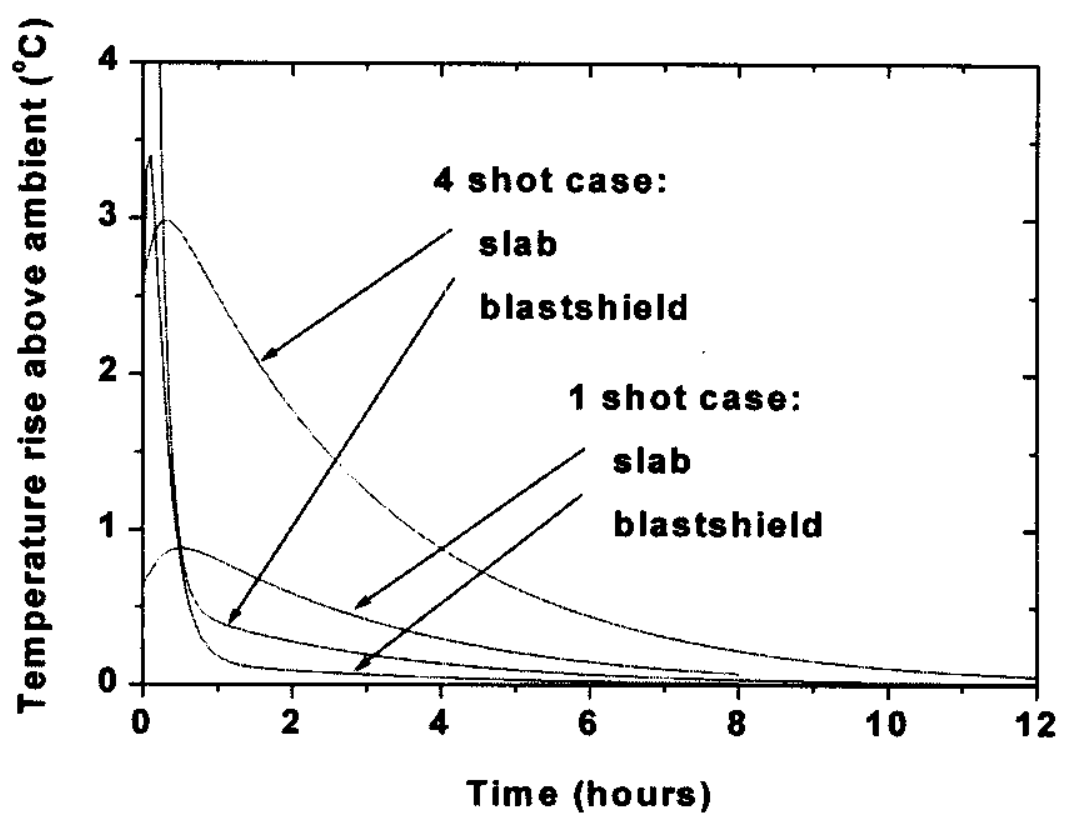

(b)

Figure 6.32. Calculated slab and blastshield temperatures for 1-shot and 4-shot cases. (a) passive flashlamp cassette cooling. (b) active flashlamp cassette cooling. 
Consider now Figure 6.31, which is for active flashlamp cooling with ambient temperature gas. In this instance, the phase distortion data is corrected to account for system effects due to the leaky blastshield seals, and it is likely to not be as accurate as for the uncooled case. The correction consisted of a root-sum-squared (rss) subtraction of the gas system component that was quantified from baseline data with fans both on and off. It is important to note that the agreement is much better for a $\beta$ factor of 1.0 , which assumes no height scaling differences between Beamlet and AMPLAB, than for a $\beta$ value of 0.5 .

With model constants now determined, Eq. (6.6) was employed to project system phase distortions for the NIF, focusing on the 0.1 wave rms limit established through the system propagation studies (see Figure 6.26). The calculated NIF system temperatures are given in Figure 6.33 for both ambient gas cooling and chilled gas cooling. The corresponding gas distortions, as extracted using Eq. (6.6) are given in Figure 6.34.. Consider first Figure 6.33, which gives predicted slab temperatures. The chilled-gas cooling cycle employed $1^{\circ} \mathrm{C}$ chilled gas for 2.5 hours, followed by immediate warming to ambient. This clearly shows from the temperature profiles the potential to achieve the 3hour recovery required for the accelerated shot rate scenario.

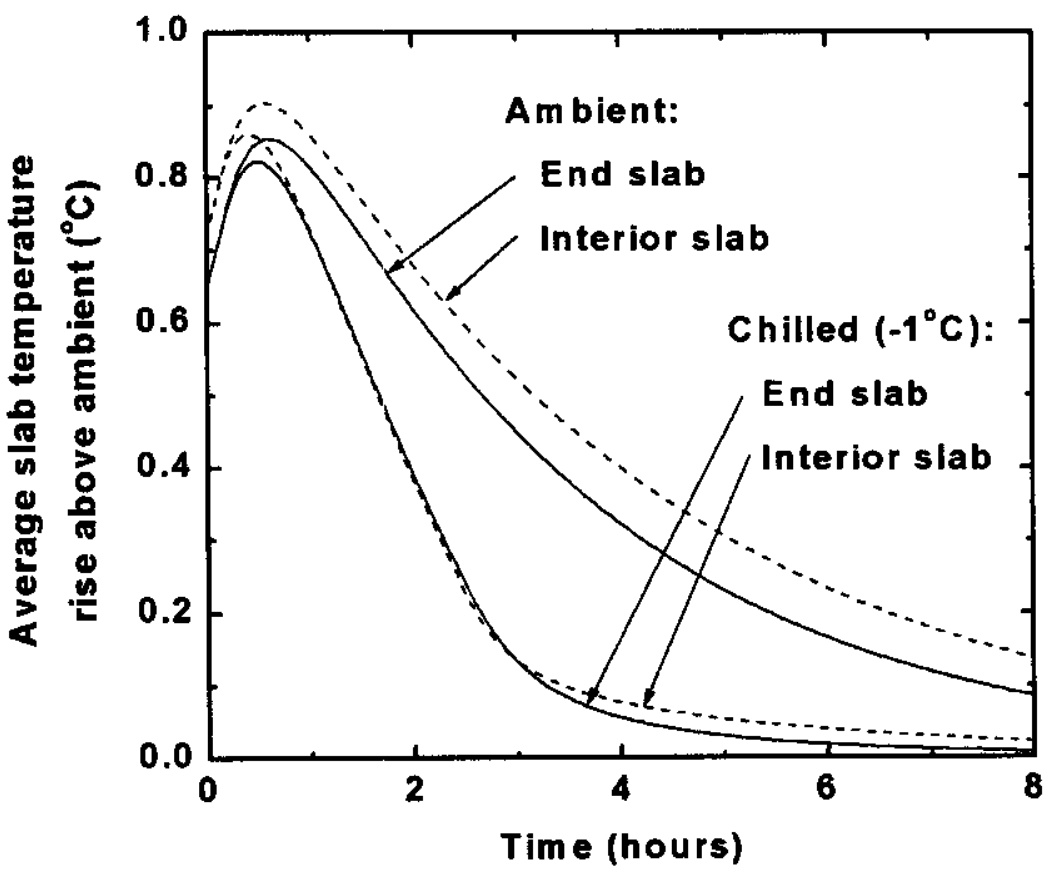

Figure 6.33. Calculated slab temperatures for the NIF amplifiers for the cases of ambient and chilled gas cooling. Predictions are given for both the interior and end slabs.

Now consider Figure 6.34. In spite of the good agreement in the AMPLAB data with a $\beta$ factor of 0.5 , both $\beta$ values are employed in an effort to bound the problem. We denote the $\beta=0.5$ line as conservative, and the $\beta=1$ line as aggressive. The NIF gas distortion limit of 0.1 waves rms, which was based on a NIF system propagation calculation, is noted on the figure. These results indicate some uncertainty at achieving the NIF distortion limit in 7 hours if the conservative assumptions are employed. Finally, 
it appears possible to achieve the required distortion level with chilled-gas cooling three hours after the start of recovery. The predicted addition to the full $80 \%$ divergence angle for the entire chain is $0.9 \pm 0.4 \mu \mathrm{rad}$ after 7 hours, and $3.3 \pm 1.7 \mu \mathrm{rad}$ after 3 hours. Note that chilled-gas cooling is a recovery option that is incorporated into the NIF cooling system design. However, chilled-gas recovery has not been optically validated. Only temperature measurements were made on the AMPLAB chilled-gas tests.

It is important to remember that this scaling model is rather crude and likely to be prone to significant inaccuracy. Thus, the absolute limits of these model bounds are very uncertain and would require additional experimental data to resolve.

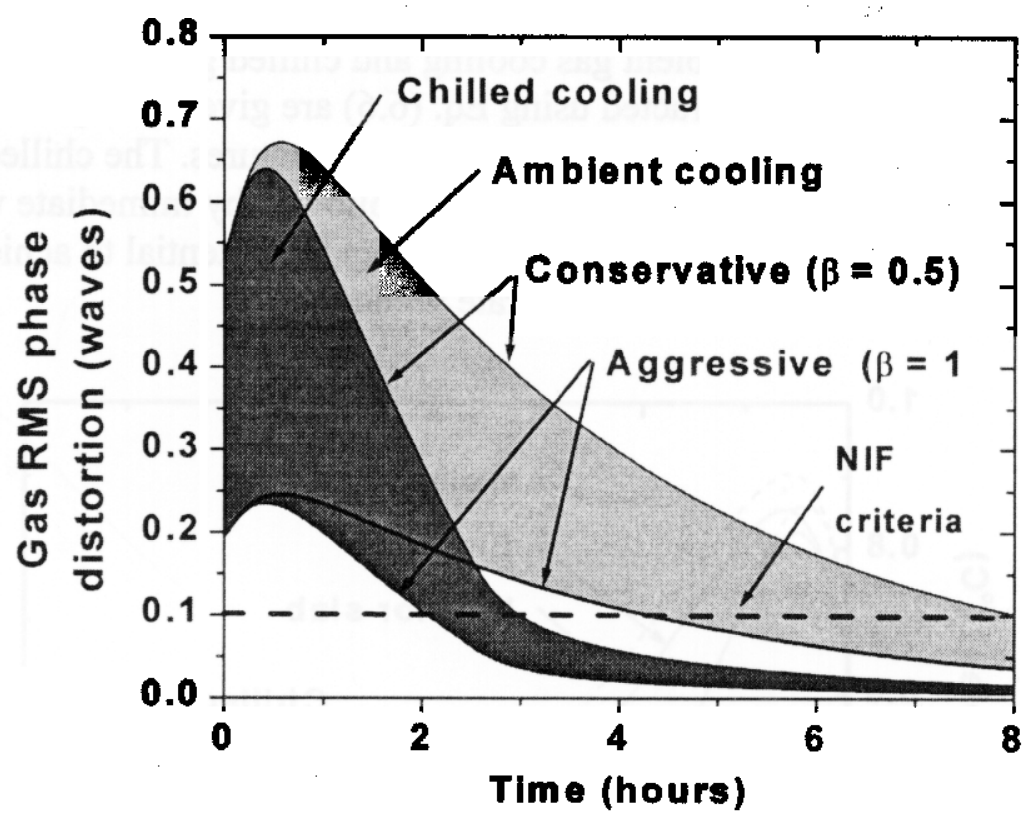

Figure 6.34. Predicted gas distortion phase disturbances in NIF for ambient and chilled gas cooling. Aggressive and conservative assumptions are used to bracket the problem.

\subsection{Summary and conclusions for thermal wavefront recovery}

In this chapter we have described the NIF thermal recovery mechanisms and criteria, described the numerical models and experiments that have been used to quantify thermal recovery, and predicted the thermal performance of the NIF amplifiers. Based on the assumptions documented in the discussion, the following conclusions are drawn.

1. Residual thermal distortions in the slabs will be reduced to acceptable levels in about four hours. Model results indicate that there is adequate margin for the 7-hour recovery case, but that results based on conservative assumptions indicate that the optical state may be marginal after 3-hours of recovery.

2. Gas-motion-induced distortions in the amplifier will meet NIF specification within, at most, eight hours, using ambient cooling gas. Slightly chilled gas 
cooling can be used to accelerate the recovery rate to meet the NIF requirements. Model results indicate that modest chilling of the cooling gas has the potential to meet the 3-hour recovery requirement for the accelerated shot-rate scenario.

3. It is important to remember that chilled-gas recovery has not been optically validated. Temperatures were measured on AMPLAB tests, but the corresponding optical measurements were not made to provide concrete 


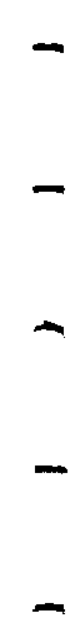




\section{Implications for NIF Performance}

The implications of AMPLAB gain files for NIF performance predictions were considered for the two cases of (1) measured AMPLAB gains and (2) predicted AMPLAB gains (per the 3-D ray trace code), assuming higher reflectivity reflectors than actually used. As these cases have slightly differing gains and gain roll-offs, they require different front-end energies and injection-pulse spatial shapes to compensate for the gain. These results show that for the NWET mission of the NIF, the output energy requirement of the regenerative amplifier in the front end is approximately two times higher for the measured (poorer reflectivity) case than for the calculated, high-reflectivity case: $3.0 \mathrm{vs} 1.6 \mathrm{~mJ}$. These are within the regen output capability of $\sim 10 \mathrm{~mJ}$. At injection to the main cavity, these numbers become 1.1 and $0.87 \mathrm{~J}$. This may imply the range over which the NIF may need to perform if the reflectors in that system start out with high reflectivity, but degrade with age. No other distinguishing differences were found between these cases for NIF system performance.

\section{Analysis}

These cases were studied using a PROP92 model of the NIF. This model was similar to that used in other studies [7.1], except that all aberrations (such as optics finishing) were eliminated to simplify analysis. It also assumed that the NIF was composed of AMPLAB slabs, which are $1 \mathrm{~mm}$ thinner than NIF slabs. The simulation represents the NWET mission of the NIF (13 ns, temporally flat), as this is the mission which causes the greatest amplifier saturation, and, hence, the greatest stress on the front-end requirements. The simulation includes the effect of beam vignetting. It also assumed a 50/50 mix of LG-750 (LHG8) and LG-770 by decreasing the gain coefficients in these files (which were all LG-770) by $6.6 \%$ for LG-750 [7.2].

The spatial shape of the front-end beam is driven both by the gain spatial shape and also by the output beam flatness. The latter effect deserves some discussion. The front-end injection beam shapes in this analysis were determined by running the code in the small-signal regime with a spatially flat input beam, and then inverting the output beam (and applying appropriate apodization). This results in an injection beam shape that gives a spatially flat beam at low-output fluences (no saturation). This simple approach has been used in past NIF simulations. It allows the same spatial beam shaper to be assumed for all NIF missions (saturating and nonsaturating). For highly saturated cases, like that studied here, however, the lower gain at the edges of the amplifier causes the edges to droop $\sim 12$ to $17 \%$ relative to the beam center on output. This is shown in Figures 7.1 and 7.2 . 


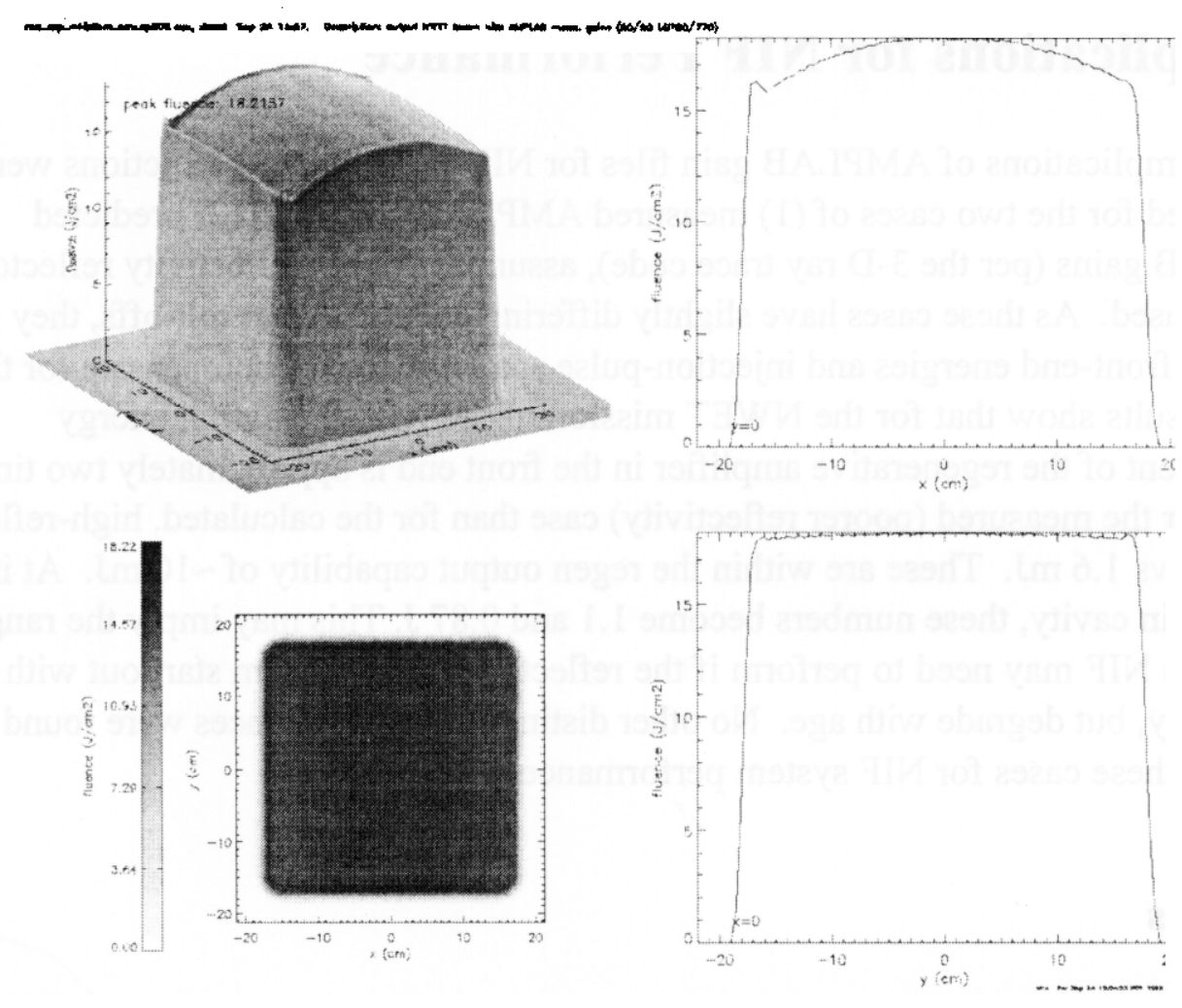

Figure 7.1. Output beam for the calculated gain case. The edge-to-eenter roll-off is $\sim 12 \%$. The output energy is $21.4 \mathrm{~kJ}$.
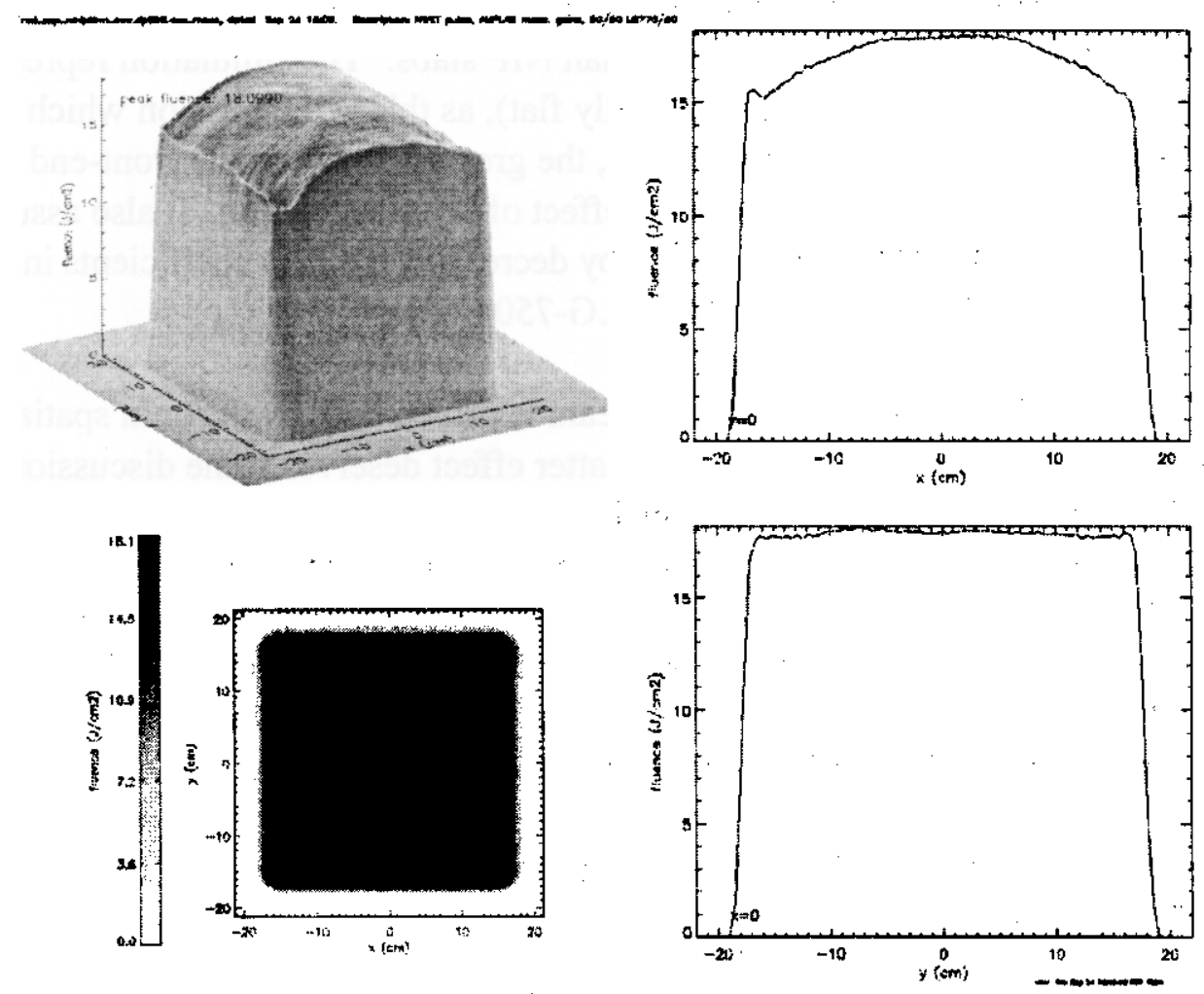

Figure 7.2. Output beam for the measured gain case. The edge-to-center roll-off is $\sim 17 \%$. The output energy is $20.5 \mathrm{~kJ}$. 
Correcting for this droop to better fill the aperture is not a compelling need and may not be feasible. When phase noise is added to the beam, the resulting modulation is significantly larger ( 30 to $40 \%$ ) than the 12 to $17 \%$ here, and so the roll-off is less apparent. Consequently, bringing all parts of the aperture up to equal risk of optics damage by high fluences is driven more by the uniformity of the aberrations on the beam than the gain roll-off. The difficulty in removing this droop lies in the highly saturated regime in which we are running for the NWET pulse. The edges of the injection beam need to be increased much more than $12 \%$, relative to the center of the beam, to correct for this droop. Indeed, trial efforts to increase this flatness have shown that a factor of two increase may be necessary. As it is the edges of the injection beam that dictate the output requirements of the regenerative amplifier (as will be discussed below), there may not be enough energy available from the regen to create a perfectly flat output beam for the NWET mission. In addition, an input spatial shape that created a flat output beam in this saturated case would create a very nonuniform output beam in the nonsaturating SSP mission. (For example, the factor of two increase referenced above would print through to the output beam, resulting in a 2:1 edge-to-center ratio there, which would be unacceptable from damage constraints.) So an effort to create a flatter beam for the NWET mission than described here would generate the need for different spatial shaping masks for the other missions.

The front-end beams for the two cases are shown in Figures 7.3 and 7.4. This is the beam leaving the regenerative amplifier and after spatial shaping (gain compensation and edge apodization). For the case of the measured gains, the fluences at the edge and center of the beam are approximately $8.2 \mathrm{e}-5$ and $0.8 \mathrm{e}-5 \mathrm{~J} / \mathrm{cm} 2$, giving a $10: 1$ spatial contrast ratio. For the calculated gains, these numbers are $4.5 \mathrm{e}-5$, and $0.6 \mathrm{e}-5 \mathrm{~J} / \mathrm{cm} 2$, giving an $8: 1$ contrast ratio. This agrees with analysis of the gain files individually, which show greater roll-off and lower average gain for the measured case (see Table 7.1).

The output energy requirement from the regenerative amplifier is driven by this pulse spatial shape in a way represented in Figure 7.5. As indicated there, the regen energy is derived from the fluences at the edges of the beam, rather than the center. (Hence, as discussed above, increasing the edges relative to the center is costly.) It is also useful to envision the spatially shaped beam as being cut out from a spatially flat-topped, square beam, which is, in turn, cut out from the Gaussian regen beam. This flat-topped beam has a fluence equal to the peak fluence at the edge of the spatially shaped beam, so one is "carving out" the center of this flat-topped pulse to do the final spatial shaping. (This is also shown in Figure 7.5.) The maximum efficiency with which this flat-topped beam can be extracted from a Gaussian beam is approximately $25 \%$. Using this, with the peak fluences of $8.2 \mathrm{e}-5$ and $4.5 \mathrm{e}-5$ given above, and a beam area of $9 \mathrm{~cm}^{2}$, we can calculate the regen output energies for the two cases. These are 3.0 and $1.6 \mathrm{~mJ}$, respectively, for the measured and calculated gain cases. This is within the nominal operating capability of the regen $(10 \mathrm{~mJ})$. The energy in the shaped beams entering the main cavity for each case is 

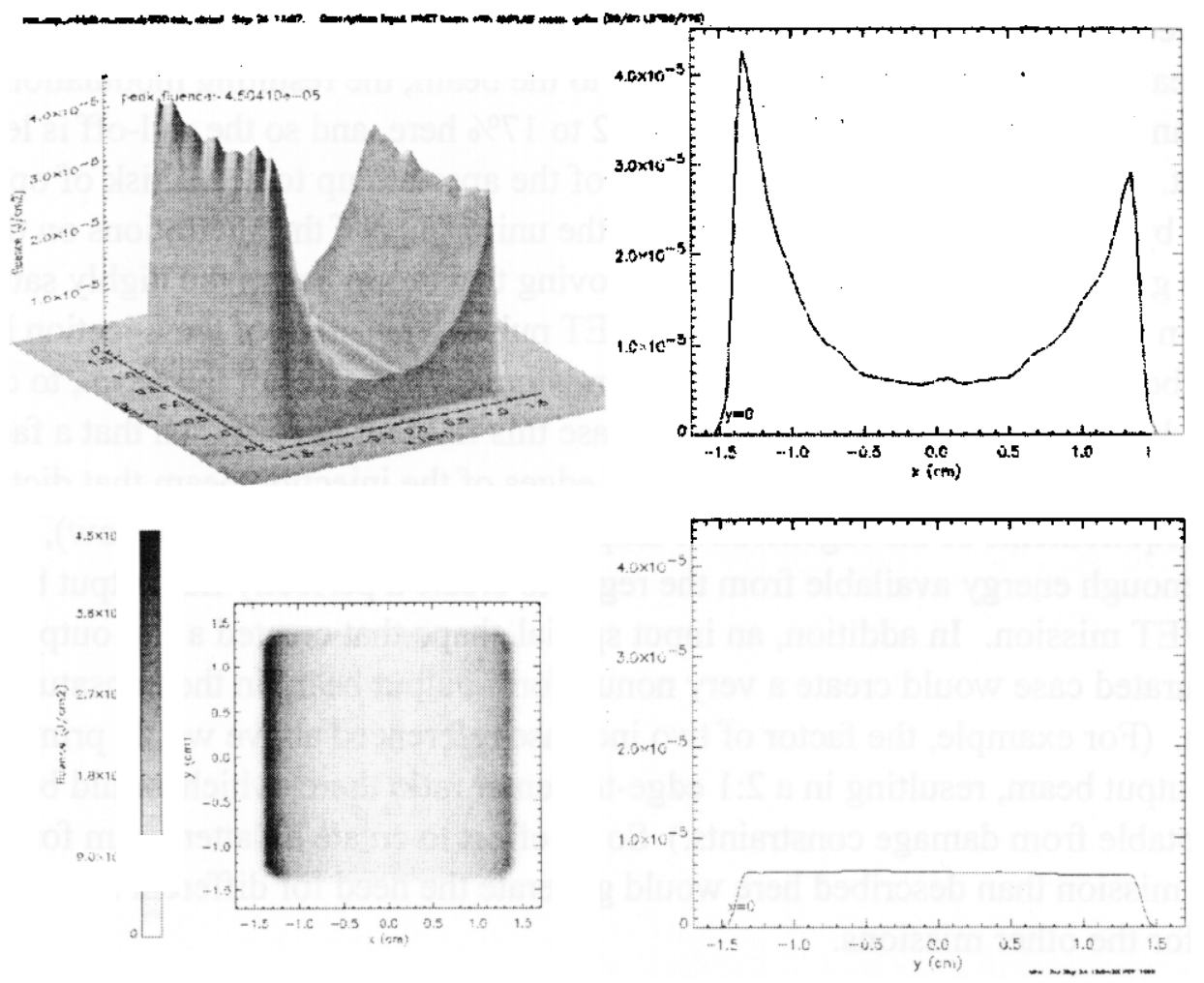

Figure 7.3. The input beam, just leaving the regen and shaping optics, for the calculated gains. The corresponding regen output beam is $\sim 1.6 \mathrm{~mJ}$.
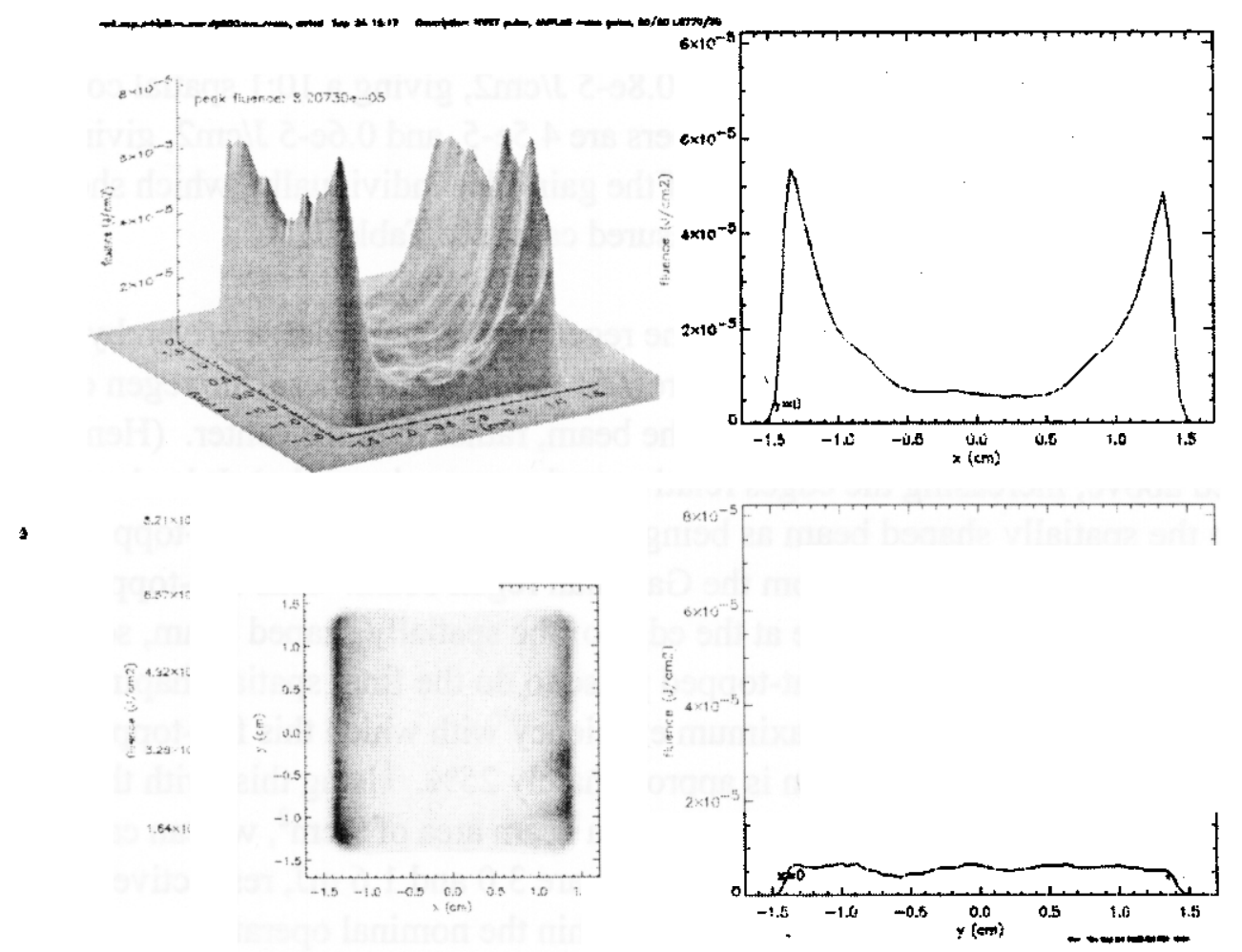

Figure 7.4. The input beam, just leaving the regen and shaping optics, for the measured gains. The corresponding regen output beam is $\sim 3.0 \mathrm{~mJ}$. 
1.1 and $0.87 \mathrm{~J}$, respectively. Again, these numbers are within the nominal output capability of the four-pass rod amplifier. The fact that an approximately factor-of-two difference in the regen output becomes only a factor of $25 \%$ difference in the four-pass outputs is due to the difference in gain shapes mentioned above: the measured slabs have more roll-off and lower gain, but the difference in roll-off (which more strongly effects the regen output) is larger than the difference in average gain (which more strongly effects the four-pass rod).

This range in regen output energies is thought to represent a change in the reflectivity of the flashlamp reflectors in NIF. In some sense, then, it may represent a performance range if the NIF reflectors are expected to start out with high reflectivity, and then degrade over time.

Table 7.1. Peak-to-edge roll-off and average gain coefficient for the six types of LG-770 slabs, as well as the chain average (including vignetting). (The term "Rotter-avg" refers to a five subaperture method of calculating average gain given in [7.2] to simulate vignetting.)

\begin{tabular}{|c|c|c|c|c|c|}
\hline \multirow[b]{2}{*}{ File } & \multirow[b]{2}{*}{ Descr } & \multicolumn{2}{|c|}{ verage gain coeff $(\mathrm{cm}-1)$} & \multicolumn{2}{|c|}{ peak-to-average } \\
\hline & & $+-20 \mathrm{~cm}$ & Rotter-avg" & $+-20 \mathrm{~cm}$ & Rotter-avg" \\
\hline FWideoc_S21.aux & damond, measured, low reflectivity'? & 0.0477 & 0.0497 & 1.1750 & 1.1290 \\
\hline 1WidwXC_S21.aux & $X$, mea sured, low reflectivity (?) & 0.0470 & 0.0490 & 1.1770 & 1.1280 \\
\hline Interior_S21.aux & interior, measured, bow refectivity (?) & 0.0534 & 0.0549 & 1.1540 & 7.1220 \\
\hline ModelOC_97_0.2.aux & dramond, calculated, high refiecivity & 0.0490 & 0.0504 & 7.1420 & 7.1080 \\
\hline ModelXC_97_0.2.aux & X, calcuated, high reliecivity & 0.0492 & 0.0511 & 7.1380 & 7.0950 \\
\hline ModellC_97_0.2.aux & interio, calcualted, high reflectivity & 0.0542 & 0.0557 & 1.0960 & 1.0650 \\
\hline 970522-1606.NIFd_s77 & damond, calculated (old, Monterey) & 0.0482 & 0.0489 & 1.0995 & 7.0837 \\
\hline 970522-1802.N1FX_s77 & X, calcuated(old, Monterey) & 0.0492 & 0.0499 & 7.0744 & 7.0590 \\
\hline 970522-1702.NIFi_s77 & interia, calcualted (od, Monterey) & 0.0512 & 0.0518 & 1.0350 & 1.0235 \\
\hline & & $+-20 \mathrm{~cm}$ & $+-19 \mathrm{~cm}$ & $+-20 \mathrm{~cm}$ & $+-19 \mathrm{~cm}$ \\
\hline$n / a$ & NIF model, measured slabs (low refl) & 0.0513 & 0.0520 & 1.1486 & 1.1325 \\
\hline$n / a$ & NIF model, calculated slabs (high reil) & 0.0523 & 0.0531 & 1.1034 & 1.0893 \\
\hline$n / a$ & ad NIF model (Monterey), calc sabs & 0.0501 & 0.0505 & 7.0406 & 7.0318 \\
\hline
\end{tabular}

energetics for three cases:

\begin{tabular}{|c|c|c|c|}
\hline & $\begin{array}{c}\text { Tegent } \\
\text { output } \\
\text { energy } \\
\text { (mJ) }\end{array}$ & $\begin{array}{c}\text { 4-pass } \\
\text { output } \\
\text { ene rgy (J) }\end{array}$ & $\begin{array}{l}\text { TW total } \\
\text { output } \\
\text { energy } \\
\text { (kJ) }\end{array}$ \\
\hline NIF model, AMPLAB measured slabs (bw refl.) & $\overline{3}$ & 1.1 & 20.5 \\
\hline NIF mode, AMPLAB calc. slabs (high retl.) & 1.6 & 0.87 & 21.4 \\
\hline NIF model, NIF modeled slabs, od(Monterey) & 7.4 & 12 & 22.6 \\
\hline
\end{tabular}




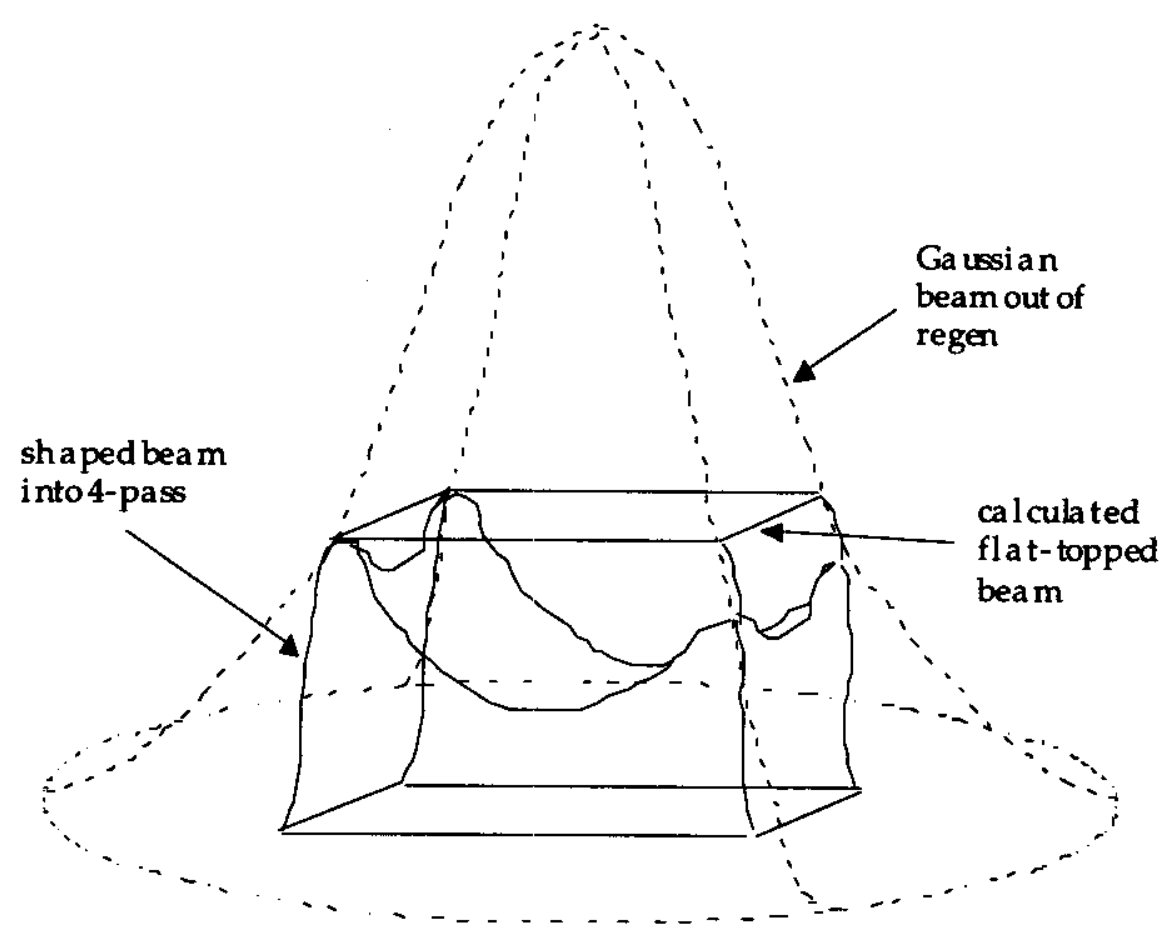

Figure 7.5. Beam shaping efficiency. The maximum efficiency (analytically) of cutting a flattopped square beam out a Gaussian regen beam is $\sim 25 \%$. The additional shaping loss to form the highly shaped beam going into the 4-pass depends on the exact gain shape. 


\section{Discussion and Recommendations}

\subsection{Gain and gain uniformity}

A model has been developed for calculating silver reflectance as a function of wavelength, angle of incidence, and polarization, which requires only normal incidence reflectance measurements for determining the thickness and refractive index of silver tarnishing. We plan to use this silver reflectance model to improve the accuracy of the 3D ray-trace gain modeling. After this has been done, and the model has been verified by modeling AMPLAB gain measurements, we recommend that action items proposed by Emmett et al [8.1] be undertaken to resolve amplifier gain issues. These recommendations, in slightly revised form, are:

- Thoroughly review and validate the methodology of the 3D-gain code. Run tests to bracket the effect of physics left out of the model, such as polarization tracking and flashlamp energy recycling.

- Determine the NIF amplifier gain and gain profiles as a function of lamp loading and glass type.

- Evaluate whether any simple modifications of NIF amplifier operation (e.g., differential loading of flashlamps) can increase focusable beam energy by either changes in gain profile or thermal distortion profile.

- Evaluate issues associated with various mixtures of glass type in the amplifier and/or amplifier chain, with the actual glass parameters as measured from pilot production material.

- Verify feasibility of protected silver by performing lifetime tests on a side-flashlamparray reflector with deep involutes.

\subsection{Prompt laser slab distortion}

The predicted prompt pump-induced wavefront distortion for a NIF beamline is about 5.5 waves, $\mathrm{P}-\mathrm{V}$, over the $\sim 35.6-\times 35.6-\mathrm{cm}$ aperture size corresponding to $50 \%$ beam intensity. The 5.5 waves is about three times greater than the 1.7 waves of prompt pumpinduced wavefront distortion measured on the Beamlet laser. Our analyses show that the AMPLAB and Beamlet results are consistent when differences in beam area, beamline architecture (NIF's has an additional pass through the booster amplifier), pump-cavity design, and measurement uncertainties are taken into account. However, the current model for prompt pump-induced wavefront distortion uses many approximations and empirically treats heating by infrared and ultraviolet sources.

In light of these observations, our recommended action items are:

- Modify the 3D ray-trace model so that it accurately predicts slab thermal loading, expressly taking into account infrared and ultraviolet sources. The measured temperature rise and small-signal gain should be used to normalize the code. 
- Model the prompt pump-induced wavefront distortion (and gain) with the flashlamp energy in the central arrays increased and the flashlamp energy in the side arrays decreased, so that pumping rates of the two different types of arrays are better balanced.

- It is conceivable that the wavefront measurements made in AMPLAB could have been influenced by flashlamp pumping of the large mirrors used to direct the probe laser beam through the amplifier. Therefore, the pump-induced distortion of the mirrors should be accurately modeled using the improved $3 \mathrm{D}$ ray-trace code.

- The predicted slab thermal distortion seven hours after a shot is $0.4 \pm 0.25$ waves, less than one-third the value allowed by the requirements. Thus, the sum of the predicted prompt pump-induced and slab thermal distortions $(5.5+0.4=5.9)$ is $20 \%$ greater than the sum of the prompt and thermal requirements $(2.7$ waves +2.2 waves $=4.9$ waves). The NIF's wavefront correction system appears to have sufficient margin to correct for this modest increase in overall distortion. We recommend that the requirements for prompt and thermal distortions be balanced to match our performance predictions.

\subsection{Long-term thermal distortion}

The predicted long-term slab and gas distortions seven hours after the shot meet the current requirements with large margins, provided the flashlamps are cooled with slightly chilled $\left(1^{\circ} \mathrm{C}\right)$ gas. Three hours after the shot, however, the predicted long-term slab and gas distortions barely meet the NIF wavefront criteria. There appears to be leverage for reducing performance risk after three hours, by:

- Cooling the flashlamps with gas that is more than $1^{\circ} \mathrm{C}$ below ambient, to accelerate recovery of the average slab temperature and to reduce gas distortion.

- Better balancing the central and side flashlamp arrays to reduce prompt pumpinduced wavefront distortion, so that more deformable mirror stroke can be allocated for correcting long-term slab distortion. Accelerating the recovery of the slab distortion would require significant and expensive design changes, such as implementing edge-cladding cooling. 


\section{References and Notes}

\section{Section 1}

1.1. NIF Subsystem Design Requirements, SSDR 1.3.2, NIF-000131-OD, Lawrence Livermore National Laboratory, Livermore, CA, January 29, 1998.

1.2. S. L. Streiff, Wavefront Error Budget Initial Estimate, NIF-0000833, Lawrence Livermore National Laboratory, Livermore, CA, October 1, 1996.

1.3. R. A. Sacks et al., "Sensitivity of NIF front end performance requirements to main amp slab properties," internal memorandum NIF-0001221, Lawrence Livemore National Laboratory, Livermore, CA, November 27, 1996.

1.4. R. Sacks and W. Williams, "NIF Performance Summary for the 11-0-5 Configuration," internal memorandum NIF-0000858, Lawrence Livermore National Laboratory, Livermore, CA, October 18, 1996.

\section{Section 2}

2.1. A. C. Erlandson, M. D. Rotter, D. N. Frank, and R. W. McCracken, "Design and Performance of the Beamlet Amplifiers," ICF Quarterly Report (5) 1, Lawrence Livermore National Laboratory, Livermore, CA, UCRL-LR-105821-95-1, pp. 18-28 (1995).

2.2. W. F. Hagen, Laser Program Annual Report -1977, Lawrence Livermore National Laboratory, Livermore, CA, UCRL-50021-77, pp. 2-228 to 2-231 (1978).

2.3. H.T. Powell, A. C. Erlandson, K.S. Jancaitis, J.E. Murray, "Flashlamp Pumping of Nd:Glass Disk Amplifiers," High-Power Solid State Lasers and Applications, Vol 1277, SPIE, Bellingham, WA, pp. 103-120 (1990).

2.4. A. C. Erlandson, K.S. Jancaitis, R. W. McCracken, M.d. Rotter, "Gain Uniformity and Amplified Spontanteous Emission in Multisegment Amplifiers," ICF Quarterly Report 92-2, Lawrence Livermore National Laboratory, Livermore, CA, UCRL-LR105821-92-3, pp. 105-114 (1992).

2.5 Replacing flat reflectors with involute reflectors in the side flashlamp array increased the average gain coefficient of a test amplifier by $5 \%$. See National Ignition Facility Project Title I Review, Amplifier Systems, NIF-0000758, Vol. 14, Lawrence Livermore National Laboratory, Livermore, CA, Oct-Nov, 1996. Bulk-gain modeling shows this $5 \%$ increase could also be achieved by increasing the flashlamp explosion fraction by $15 \%$, from 0.20 to 0.23 . 
2.6. J. H. Kelly, M. J. Shoup III, M. D. Skeldon, and S. T. Bui, "Mechanical Design of 15and 20-cm Clear-aperture Disk Amplifiers for the OMEGA Upgrade, in Solid State Lasers III, edited by G. J. Quarles, Proceedings Vol. 1627, SPIE, Bellingham, WA, pp. 252-259 (1992).

2.7. A. C. Erlandson, H. Lambert, L.E. Zapata, L. Pedrotti, D.W. Larson, M.D. Rotter, W. J. Dallum, S. E. Seznec, G. LeTouze, E. Grebot, O. Carbourdin, J. C. Fornerod, and P. Bosch, "The effect of amplifier component maintenance on laser system availability and reliability for the US National Ignition Facility," Second Annual Conference on Solid State Lasers for Application to Inertial Confinement Fusion, Proceedings Vol. 3047, SPIE, Bellingham, WA, pp. 16-37 (1997).

2.8. MIL-STD-1246C, Product Cleanliness Levels and Contamination Control Program, U.S. Department of Defense, Defense Standardization Program Office, Falls Church, VA, March 1997.

\section{Section 3}

3.1. Ken Jencaitis, private communication.

3.2. Mark Rotter et al., "Flashlamp cassette positions in the NIF amplifiers," internal memorandum NIF-0004010, Lawrence Livermore National Laboratory, Livermore, CA, December 1, 1997.

3.3. J. F. Mengue, Shaping of the Reflectors and Gain of the ADL amplifiers, UCRLUR-127403, Lawrence Livermore National Laboratory, CA, May 16. 1997.

\section{Section 4}

4.1. K. S. Jancaitis, S. W. Haney, D. H Munro, G. LeTouzé, O. Cabourdin, SPIE Proc. Vol. 3047, pp. 106-111 (1997).

4.2. G. LeTouzé et al., "3-D Gain Modeling of the LMJ and NIF Amplifiers", Solid State Lasers for Application to Inertial Confinement Fusion, Third International Conference 1998 (to be published).

4.3. H. T. Powell, A. C. Erlandson, K. S. Jancaitis, SPIE Proc. Vol. 609, pp. 78-94 (1986).

\section{Section 5}

5.1. J. E. Murray, W. F. Hagen, and B. W. Woods, "Flashlamp-Induced Aberrations in Disk Amplifiers," Laser Program Annual Report, UCRL-50021-82, LLNL, Livermore, CA. pp.7-72, (1982). 
5.2 M. Takeda, H. Ina, and S. Kobayashi, "Power-transform method of fringe-pattern analysis for computer-based topography and interferometry," J. Opt. Soc. Am., Vol. 72, no. 1, January, 1982.

5.3. F. J. Harris, "On the Use of Windows for Harmonic Analysis with the Discrete Fourier Transform," Proceedings of IEEE, Vol. 66, no. 1, January, 1978.

5.4. M. Henesian, private communication.

5.5. G. LeTouzé, Olivier Cabourdin, J.F. Mengue, M. Rotter and K. Jancaitis. "Shaped reflectors for Pump Cavities," 2nd Annual Conf. Solid State Lasers for Application to ICF, Limeil, France, 1996.

5.6. A. Shapiro, TOPAZ3D - A 3-D Finite Element Heat Transfer Code, UCID-20484, LLNL, Livermore, CA., Aug. 1985.

5.7. B. N. Maker, NIKE3D - A Nonlinear, Implicit 3-D Finite Element Code for Solid and Structural Mechanics, UCRL-MA-105268, Rev. 1, LLNL, Livermore, CA., April 1995.

5.8. S. Doss and R. Gelinas, "Mathematics and Physics of the BREW Code," Laser Program Annual Report, UCRL-50021-86, LLNL, Livermore, CA., pp. 7-132, (1986).

5.9. S. E. Stokowski, R. A. Saroyan, and M.J. Weber, Laser Glass - Nd-doped Glass Spectroscopic and Physical Properties, V1, M-095, Rev. 2, Vol. 1, LLNL, Livermore, CA., (1981).

5.10. J. Campbell, " 25 years of laser glass development leading to a $1.8 \mathrm{MJ}, 500 \mathrm{TW}$ laser for fusion ignition," 18th International Congress on Glass, San Francisco, CA, July $5-10,1998$.

5.11. M. Born and E. Wolf, Principles of Optics, $6^{\text {th }}$ Ed., p. 122, Pergamon Press, New York, (1980).

5.12. R. M. A. Azzam and N. M. Bashara, Ellipsometry and Polarized Light, p. 488, North-Holland, Amsterdam, (1987).

5.13. B. Boley, and J. Weiner, Theory of Thermal Stresses, Ch. 12, J. Wiley \& Sons, New York, (1960).

5.14. P. Wegner et al., "Measurements of Beamlet wavefront and divergence," internal memorandum LST-BLT97-068/pw, Lawrence Livermore National Laboratory, Livermore, CA, April 29, 1997.

5.15. J. Lawson, K. Manes, C. Marshall, M. Rotter, and S. Sutton, Issues on NIF Amplifier Wavefront Performance - Rationale for a 'Null' Test, NIF-0012333, Lawrence Livermore National Laboratory, Livermore, CA, Aug. 1998. 
5.16. W. Williams, private communication.

\section{Section 6}

6.1. S. Sutton, Flashlamp Cooling Flow Rate Considerations for NIF-A Preliminary Assessment, LST-ALC96-071, August 26, 1996.

6.2. J. D. Maltby and P. J. Burns, Users's Manual for MONTE2D and MONTE3D Monte Carlo Radiative Exchange Factor Codes, UCRL-MA-112192, February 1993.

6.3. A. B. Shapiro, TOPAZ3D-A three-dimensional finite element heat transfer code, LLNL, UCID-20484, August 1985.

6.4. B. N. Maker, et al., NIKE3D - A nonlinear, implicit, three-dimensional finite element code for solid and structural mechanics, LLNL, UCRL-MA-105268, Rev. 1, April 1995.

6.5. The optical propagation code used in this study is under continuing development at LLNL. The principle authors of this code are W. Neumann and M. Rotter.

6.6. R. E. Pierce, Three-dimensional thermal modeling of the NIF amplifiers" report in preparation.

6.7. A. B. Shapiro, TOPAZ2D - A Two-Dimensional Finite Element Code for Heat Transfer Analysis, Electrostatic, and Magnetostatic Problems, UCID-20824, July 1986.

6.8. R. Sacks, et al., "PROP92 Fourier Based Propagation Code," 1996 ICF Annual Report, UCRL-LR-105821-96, p. 207.

6.9. S. Sutton, W. Williams, K. Manes, R. London, A simple scaling model for projecting NIF gas distortion optical distortions from AMPLAB and Beamlet data, NIF-0013709, Lawrence Livermore National Laboratory, Livermore, CA, September 1998.

\section{Section 7}

7.1. NIF Laser System Performance Ratings, UCRL-ID-131115, Lawrence Livermore National Laboratory, Livermore, CA, (1998).

7.2. M. Rotter and M. Guenet, NIF-0002700, Lawrence Livermore National Laboratory, Livermore, CA, 


\section{Section 8}

8.1. J. Y. Emmett et al., "NIF Amplifier Technology Action Items," internal memorandum NIF-13716, Lawrence Livermore National Laboratory, Livermore, CA, September 11, 1998.

\section{Appendix A}

A.1. K. Jancaitis, "Flashlamp Modeling," Laser Program Annual Report, UCRL 5002186, LLNL, Livermore, CA. pp. 6-3, (1986).

A.2. J. P. Markiewicz and J.L. Emmett, "Design of Flashlamp Driving Circuits," IEEE J.Q.E., QE-2, p. 707, (1966).

A.3. H. T. Powell, A.C. Erlandson, K.S. Jancaitis, and J.E. Murray, "Flashlamp Pumping of Nd:Glass Disk Amplifiers," SPIE 1277, p. 103, (1990).

A.4. K. S. Jancaitis, "Disk Amplifier Performance Model." Laser Program Annual Report, UCRL-LR-105820-88/89, LLNL, Livermore, CA., pp. 6-12, (1993). 


\section{Appendix A}

\section{Calculation of Slab Temperature Rise Due to Absorption of 400- to 1000-nm Pump Light}

In general, the distribution of thermal power deposited in the laser slab by flashlamp pump light, $\mathrm{Q}$, is an arbitrary function of position and time. For purposes of this model, however, we have assumed a separable source function, i.e.,

$$
Q(x, y, z, t)=A s(x)\left[g_{0}(y) f(z)+g_{h}(y) f(h-z)\right] u(t)+Q_{e c},
$$

where $\mathrm{x}$ and $\mathrm{y}$ are vertical and horizontal coordinates in the plan of the slab, respectively; $\mathrm{z}$ is the coordinate running through the thickness of the slab; $\mathrm{h}$ is the thickness of the slab; $\mathbf{s}(\mathrm{x})$ denotes the vertical variation of the pump profile, which is assumed to be unity for both the front and back surfaces of the slab; $g_{0}(y)$ and $g_{h}(y)$ denote the horizontal variation of the pump profile at $z=0$ (the front surface of the laser slab) and $x=h$ (the back surface), respectively; $f(z)$ denotes the pump profile through the thickness of the slab; $u(t)$ denotes the time dependence of the heat deposition; $A$ is a constant multiplier; and $\mathrm{Q}_{\mathrm{ec}}$ is the thermal source term for the edge cladding. Each of these terms is discussed below.

In the multisegment amplifiers envisioned for use in the NIF, the flashlamps are oriented vertically, and there are silver-coated metal reflectors at the top and bottom of the pump cavity. These features are indicative of the vertical symmetry that minimizes variations in pump light fluence in the vertical direction and allows us to approximate the function $\mathrm{s}(\mathrm{x})$ with a constant. In reality, the reflectors are not perfect, and so there is a $\sim 1-2 \%$ roll-off in pump light at the top and bottom of the pump cavity. This roll-off could have a small effect on pump-induced wavefront distortion, as noted below.

We used a 2D+ ray-trace code called Ampmodel [5.5] to calculate the pump light distributions across the long, horizontal dimension of the laser slab. Although Ampmodel performs ray-tracing in the horizontal plane, the horizontal pump-light distributions predicted with Ampmodel are very close to distributions predicted with the 3D ray-trace code described in Section 3. Figure A.1 shows pump light distributions calculated with Ampmodel for the NIF prototype amplifier in the two-slab-long diamond configuration. These distributions were used by TOPAZ3D for the functions $g_{0}(y)$ and $g_{h}(y)$. Linear interpolation was used to determine $g_{0}(y)$ and $g_{h}(y)$ for arbitrary values of $y$.

The thermal energy deposition through the thickness of the slab was calculated using the LLNL Xe flashlamp model [A.1]. This model calculates the spectrally integrated Ndpumping rate for different slab thickness, Nd-doping concentrations, and flashlamp input powers. The calculated profile calculated with Amplab input parameterswas approximated with the double exponential function

$$
f(z)=e^{-\mu}{ }_{1}+c e^{-\mu}{ }_{2},
$$


where $\mu_{1}, \mu_{2}$, and $c$ are the fit coefficients. Over the range of explosion fractions considered ( 0.2 to 0.23 ), the values of $\mu_{1}$, and $\mu_{2}$ are insensitive to explosion fraction. A plot of the energy deposition profile and the corresponding fit is shown in Figure A.2.

The deposition profile was slightly skewed away from the surface, however, as the calculation was performed using normal-incidence rays. This uncertainty is accounted for by normalizing the predictions to measurements, by adjusting the constant, $\mathrm{A}$.

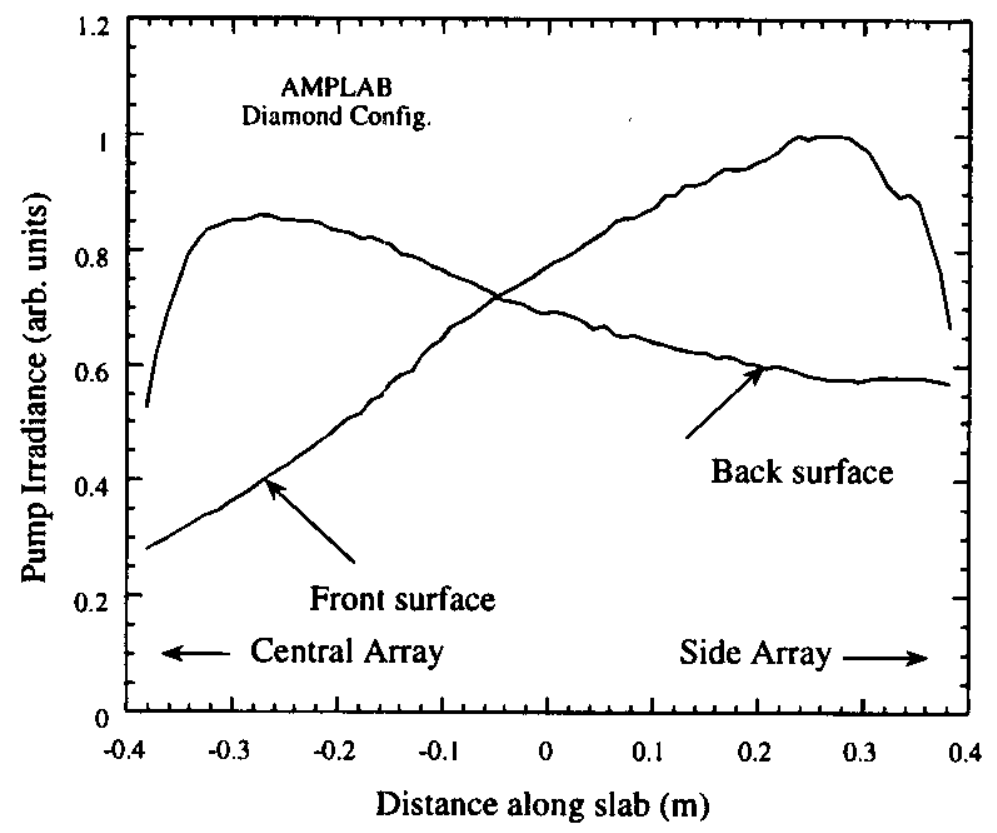

Figure A.1. Pump irradiance profiles-AMPLAB diamond configuration.

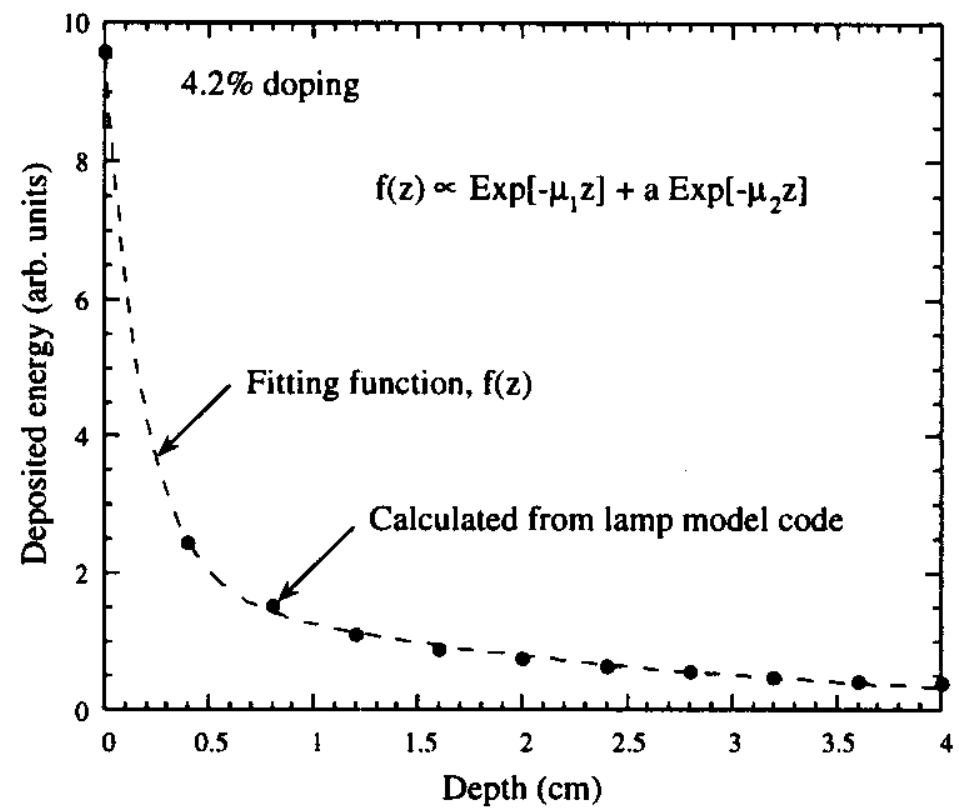

Figure A.2. Energy deposition profile through slab (•) and fit (---) for normal incidence flashlamp light in the 400- to 1000-nm pump bands. 
To describe the temporal behavior of the pump pulse, we use an analytic expression for $u(t)$. We first need to describe the temporal behavior of the electrical input power to the lamp. Since the flashlamp is a nonlinear circuit element, the actual pulse shape is described by a nonlinear differential equation [A.2]. We have found, however, that an excellent approximation to the shape is given by the function

$$
p(t) \propto t \operatorname{Exp}\left[-(t-a)^{2} / \tau^{2}\right],
$$

where $a$ and $\tau$ are fit parameters. A plot of the electrical input power as determined from a numerical integration of the circuit equation and the approximation given by Eq. (A.3) is shown in Figure A.3. The optical output power, $u(t)$, may be calculated using the nonlinear equation

$$
d w d t=[\eta(u) p(t)-u(t)] / \tau_{R},
$$

where $\eta(u)$ is the instantaneous radiant efficiency of the flashlamp (corrected for arcexpansion effects), and $\tau_{R}$ is radiative recombination time of the plasma (approx. $30 \mu \mathrm{s}$ ) [A.4]. We have found, however, that $\eta(u)$ can be accurately approximated by the expression

$$
\eta(u)=.653+2.33 \times 10^{-4} \tau_{10}, \quad\left(200<\tau_{10}<500 \mu \mathrm{s} 0.15<\mathrm{f}_{\mathrm{x}}<0.25\right)
$$

where $\tau_{10}$ is the full-width, tenth-max time of the electrical input power pulse. In Figure A.4 we show the comparison between the numerical solution to Eq. (A.4) (with $\eta(u)$ as given by Ref. 7) and the solution using the approximation given by Eq. (A.5). We see that over the time range specified, the agreement is quite good. Using Eqs. (A.3)(A.5), an analytic expression for $u(t)$ may be obtained.

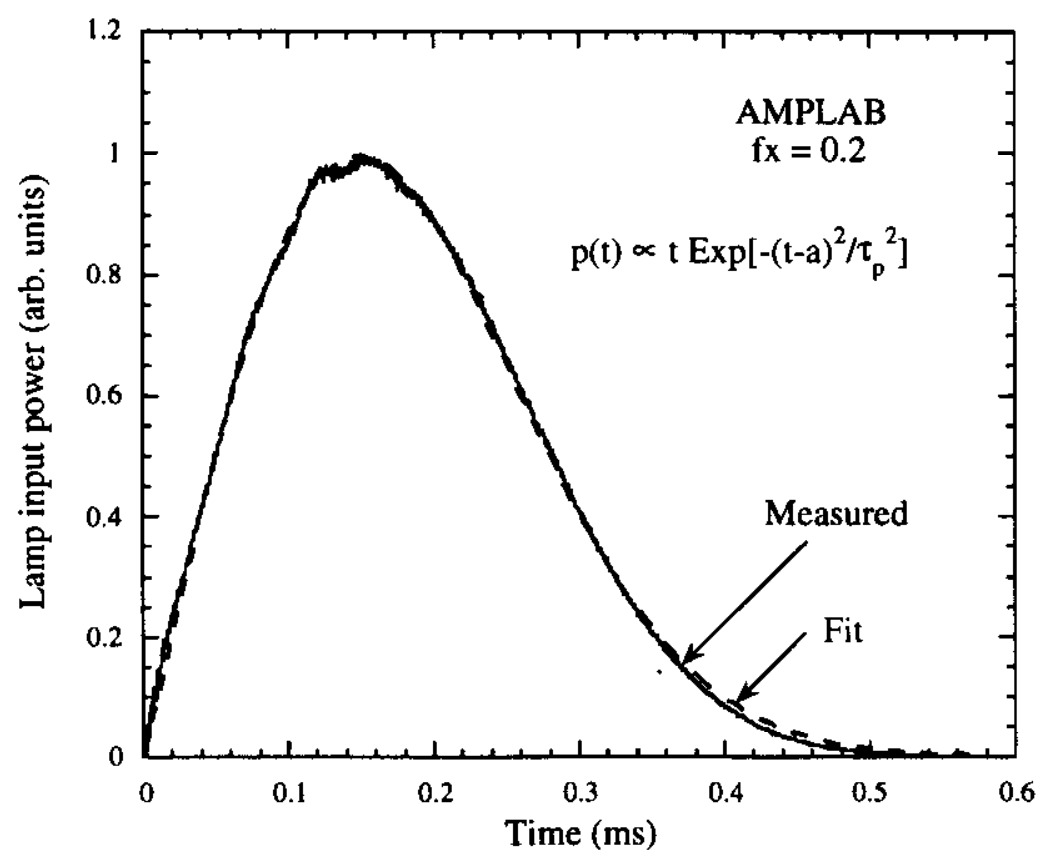

Figure A.3. Measured electrical input power to lamp (-) and fit (---). 

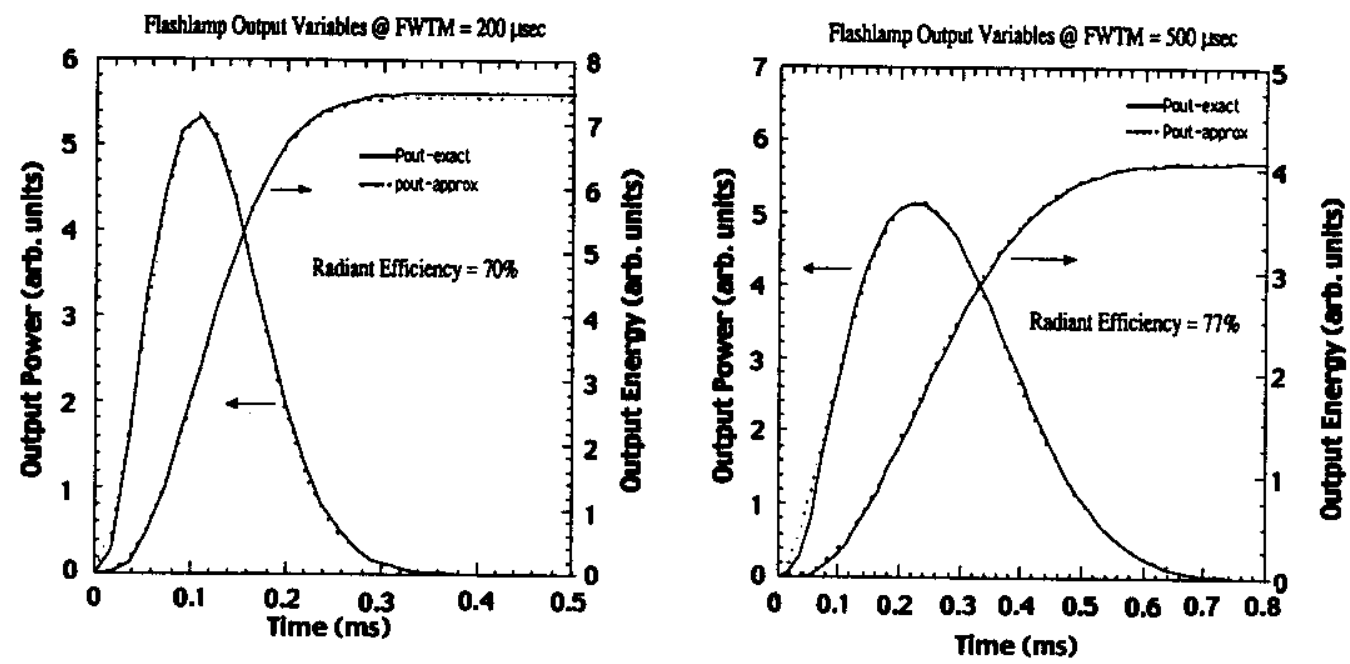

Figure A.4. comparison of exact and approximate solutions to the flashlamp output equation.

The scale factor, A in Eq. (A.1), was adjusted to give good agreement between predicted and measured prompt pump-induced wavefront distributions. After $\mathrm{A}$ had been determined from measurements made in AMPLAB, the same value of $A$ was used for predicting the performance of the NIF amplifiers.

The term $Q_{e c}$, which represents the heat deposited in the edge claddings, was calculated using

$$
\mathrm{Q}_{\mathrm{ec}}=\mathrm{Q}_{\mathrm{ec}, \mathrm{pump}}+\mathrm{Q}_{\mathrm{ec}, \mathrm{ASE}} \text {, }
$$

where $Q_{\mathrm{ec}, \text { pump }}$ represents the heat deposited into the edge claddings by flashlamp pump light, and $\mathrm{Q}_{\mathrm{ec}, \mathrm{ASE}}$ represents the heat deposited into the edge claddings by the ASE. The source term is broken up in such a manner because the time dependence of the two parts

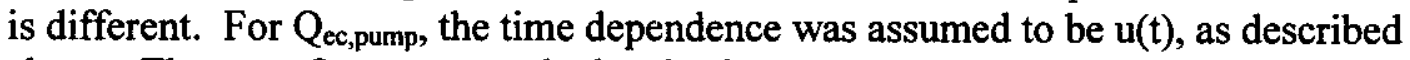
above. The term $Q_{e c, A S E}$ was calculated using

$$
\mathrm{Q}_{\mathrm{ec}, \mathrm{ASE}} \propto \phi(\mathrm{t}) \beta \Gamma \mathrm{e}^{-\beta \mathrm{x}},
$$

where $\beta$ is the edge-cladding absorption coefficient at $1.053 \mu \mathrm{m}, \Gamma$ is the fluence of the incident ASE, and $\phi(t)$ gives the time dependence of the incident ASE. After determining the time dependent stored energy density $\rho(t)$ using the bulk gain model [A.3], we calculated $\phi(t)$ using the expression

$$
\phi(t) \propto \rho(t)[a+b\{\operatorname{Exp}[\rho(t)]-1\}],
$$

where $\mathrm{a}$ and $\mathrm{b}$ are constants. See reference A. 4 for the derivation. 
At present, we do not have an accurate ab initio calculation of the ASE fluence on the edge cladding. However, based on measurements with the Beamlet laser, we estimate a fluence of $4 \mathrm{~J} / \mathrm{cm}^{2}$ for ASE and another $2.5 \mathrm{~J} / \mathrm{cm}^{2}$ due to the pump light.

Equations (A.1)-(A.8) are used in TOPAZ3D to determine the temperature distribution in the laser slab. Due to the shortness of the pump pulse (a few hundred $\mu \mathrm{sec}$ ), adiabatic boundary conditions are used on all faces of the slab. 


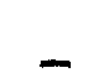

